\title{
Kolmogorov's Theorem for Low-Dimensional Invariant Tori of Hamiltonian Systems
}

\author{
Plotnikov P.I., Kuznetsov I.V.
}

Lavrentyev Institute of Hydrodynamics Siberian Branch of RAS

Address: Lavrentyev pr.15 Novosibirsk 630090, Russia,

Novosibirsk State University

Address: Pirogova st. 2, Novosibirsk 630090, Russia

\begin{abstract}
In this paper the problem of persistence of invariant tori under small perturbations of integrable Hamiltonian systems is considered. The existence of one-to-one correspondence between hyperbolic invariant tori and critical points of the function $\Psi$ of two variables defined on semi-cylinder is established. It is proved that if unperturbed Hamiltonian has a saddle point, then under arbitrary perturbations there persists at least one hyperbolic torus.
\end{abstract}

Key words: invariant tori; KAM theory; Percival's variational principle. MSC2010 numbers: 37J40, 70H08, 70H15, 70H30, 70K43

\section{Contents}

1 Notation 2

2 Problem formulation. Results 4

3 Basic group of of canonical transformations 8

4 A modified problem and bifurcation equations 14

*plotnikov@hydro.nsc.ru 
5 Linear Problem 24

5.1 Approximate equations . . . . . . . . . . . . 25

5.2 Differential of $\boldsymbol{\Phi}$. Approximate inverse . . . . . . . . . . . 28

6 Implicit function theorem 35

6.1 Nash-Moser-Zehnder Implicit Function Theorem . . . . . . . . 35

6.2 Solvability of the main operator equation . . . . . . . . 38

7 Dependence on parameters. Jacobi vector fields 42

7.1 Differentiability with respect to parameters . . . . . . . . . 42

7.2 Representation of derivatives. Jacobi vector fields . . . . . . . 45

8 Linear problem 49

8.1 Preliminaries. Sobolev spaces . . . . . . . . . . . . . . 49

8.2 Basic linear problem. . . . . . . . . . . . 50

9 Quadratic form

10 Action functional. Proof of Theorem 2.2

10.1 Proof of Theorem $2.2 \ldots \ldots \ldots$. . . . . . . . . . . 71

A Proof of Theorem 3.1 76

B Proof of Theorem 3.2

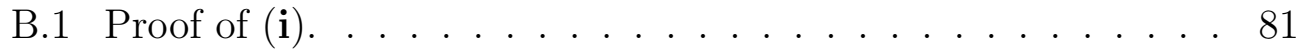

B.2 Proof of $(\mathbf{i i}) \ldots \ldots \ldots \ldots$

C Proof of Lemma 4.1

D Proof of Theorem 4.7

E Proof of Theorems 5.1 and $8.1 \quad 101$

E.1 Proof of Theorem 5.1 . . . . . . . . . . . . . . . . . . . . 101

E.2 Proof of Theorem 8.1 . . . . . . . . . . . . . . . . . . . . . 119

\section{Notation}

First, we introduce some notation which is used troughout of the paper. Every vector $\mathbf{v}$ in $m$-dimensional Euclidean space is assumed to be a column 
vector. Row vectors are denoted by $\mathbf{v}^{\top}$. Hence $\mathbf{v}=\left(v_{1}, \ldots, v_{m}\right)^{\top}$. For a matrix $\mathbf{A}=\left(A_{i j}\right), i, j=1, \ldots, n$, with entries $A_{i j}, i$ is the row index and $j$ the column index, and $\mathbf{A}^{\top}=\left(A_{j i}\right)$ stands for the transposed matrix. The product of a matrix $\mathbf{A}$ by a column vector $\mathbf{v}$ is denoted by $\mathbf{A v}$. It is the column vector with the components $(\mathbf{A v})_{i}=A_{i j} v_{j}$. The product of a row vector $\mathbf{v}^{\top}$ and a matrix $\mathbf{A}$ is the row vector with the components $\left(\mathbf{v}^{\top} \mathbf{A}\right)_{j}=v_{i} A_{i j}$. We thus get $\mathbf{v}^{\top} \mathbf{A}=\left(\mathbf{A}^{\top} \mathbf{v}\right)^{\top}$. The product of two matrices $\mathbf{C}=\mathbf{A B}$ is a matrix with the entries $C_{i j}=A_{i k} B_{k j}$ with the summation convention over the repeated indices. The scalar product of two vectors is $\mathbf{v}^{\top} \cdot \mathbf{u}=v_{i} u_{i}$. By abuse of notation, we also denote by $\mathbf{u} \cdot \mathbf{v}=u_{i} v_{i}$ the scalar product of two column vectors.

The tensor product of two vectors $\mathbf{u}, \mathbf{v} \in \mathbb{R}^{n}$ is the matrix $A:=\mathbf{u} \otimes \mathbf{v}$ with the entries $A_{i j}:=u_{i} v_{j}, i, j=1, \ldots, n$. For the product of this matrix with a vector we have

$$
(\mathbf{u} \otimes \mathbf{v}) \mathbf{w}=\left(\mathbf{v}^{\top} \cdot \mathbf{w}\right) \mathbf{u} \quad \text { and } \quad \mathbf{w}^{\top}(\mathbf{u} \otimes \mathbf{v})=\left(\mathbf{w}^{\top} \cdot \mathbf{u}\right) \mathbf{v} .
$$

There is a difference between the Jacobian $\mathbf{v}^{\prime}(x)$ of a vector function $\mathbf{v}$ : $\mathbb{R}^{n} \rightarrow \mathbb{R}^{m}$ and its gradient: the Jacobian is denoted by

$$
\mathbf{v}^{\prime}:=\left(\partial_{x_{j}} v_{i}\right)=\left[\frac{\partial \mathbf{v}}{\partial x_{1}}, \frac{\partial \mathbf{v}}{\partial x_{2}}, \ldots, \frac{\partial \mathbf{v}}{\partial x_{n}}\right],
$$

and the gradient is its transpose

$$
\nabla \mathbf{v}=\mathbf{v}^{\prime \top}=\left(\partial_{x_{i}} v_{j}\right)=\left[\nabla v_{1}, \nabla v_{2}, \nabla v_{3}\right]
$$

If a function $\mathbf{v}: \mathbb{R}^{n-k} \times \mathbb{R}^{k}$ depends on two variables $y \in \mathbb{R}^{n-k}$ and $z \in \mathbb{R}^{k}$, then

$$
\mathbf{v}_{y}^{\prime}(y, z)=\left(\partial_{y_{j}} v_{i}\right)_{i j}=\left[\frac{\partial \mathbf{v}}{\partial y_{1}}, \frac{\partial \mathbf{v}}{\partial y_{2}}, \ldots, \frac{\partial \mathbf{v}}{\partial y_{n-k}}\right],
$$

and $\nabla_{y} \mathbf{v}=\left(\mathbf{v}_{y}^{\prime}\right)^{\top}$. For the derivatives of a scalar function $H$ we will use the notation

$$
\frac{\partial H}{\partial y}(y, z)=H_{y}^{\prime}(y, z) \text { and } \frac{\partial^{2} H}{\partial z \partial y}(y, z)=\nabla_{z}\left(H_{y}^{\prime}\right)(y, z) \text {. }
$$

In other words, $\partial^{2} H / \partial z \partial y$ is the matrix with the entries $\partial^{2} H / \partial z_{i} \partial y_{j}$ and

$$
\frac{\partial^{2} H}{\partial z \partial y}=\left(\frac{\partial^{2} H}{\partial y \partial z}\right)^{\top}
$$


Let $\varrho>0$. Denote by $\Sigma_{\varrho}$ the complex neighborhood

$$
\Re_{\varrho}=\left\{\boldsymbol{\xi} \in \mathbb{T}^{n-1}+i \mathbb{R}^{n-1}: \operatorname{Re} \xi \in \mathbb{T}^{n-1},\left|\operatorname{Im} \xi_{j}\right| \leq \varrho, \quad 1 \leq j \leq n-1\right\}
$$

of the (n-1)-dimensional torus $\mathbb{T}^{n-1}$. We will identify $\Re_{\varrho}$ with the strip

$$
\left\{\boldsymbol{\xi}: \operatorname{Re} \xi \in \mathbb{R}^{n-1},\left|\operatorname{Im} \xi_{j}\right| \leq \varrho, \quad 1 \leq j \leq n-1\right\} \subset \mathbb{C}^{n-1} .
$$

We denote by $B_{o}^{n}$ the complex neighborhood

$$
B_{\varrho}^{n}=\left\{\boldsymbol{p} \in \mathbb{C}^{n}:|\operatorname{Re} \boldsymbol{p}| \leq \varrho \in D,\left|\operatorname{Im} p_{j}\right| \leq \varrho, \quad 1 \leq j \leq n\right\} \subset \mathbb{C}^{n}
$$

\section{Problem formulation. Results}

Invariant tori. In this paper we consider the problem of persistence of quasi-periodic motions spanning lower dimensional tori in a nearly-integrable Hamiltonian system

$$
\dot{\boldsymbol{p}}=-\partial_{\boldsymbol{q}} H(\boldsymbol{p}, \boldsymbol{q}), \quad \dot{\boldsymbol{q}}=\partial_{\boldsymbol{p}} H(\boldsymbol{p}, \boldsymbol{q})
$$

with the Hamiltonian

$$
H(\boldsymbol{p}, \boldsymbol{q})=H_{0}(\boldsymbol{p})+\varepsilon H_{1}(\boldsymbol{p}, \boldsymbol{q}) .
$$

Here the functions $H_{0}$ and $H_{1}$ are analytic on the set $D \times \mathbb{T}^{n}$, where $D \subset \mathbb{R}^{n}$ is a neighborhood of zero and $\mathbb{T}^{n}=\mathbb{R}^{n} / 2 \pi \mathbb{Z}^{n}$ is $n$-dimensional torus. In particular, $H_{1}$ is an analytic $2 \pi$-periodic function of the angle variable $\boldsymbol{q}=$ $\left(q_{1}, \ldots, q_{n}\right)$. For $\varepsilon=0$ the system is integrable and the phase space is foliated by invariant $n$-dimensional invariant tori $\{\mathbf{q}=\overline{\boldsymbol{\omega}} t+$ const., $t \in \mathbb{R}\}$ with the frequency vectors $\overline{\boldsymbol{\omega}}=\nabla H_{0}(\boldsymbol{p}), \boldsymbol{p} \in D$. If all components of $\overline{\boldsymbol{\omega}}$ are rationally independent, then KAM theory, see [11] and [1], shows that, under suitable non-degeneracy assumptions, such invariant tori persist under small analytic perturbations.

An invariant $n$-dimensional invariant torus of the unperturbed system is said to be resonance if the number of rationally independent components

of $\overline{\boldsymbol{\omega}}$ is $m<n$. A resonance invariant torus of the unperturbed system is foliated by invariant $m$-dimensional tori. A resonance torus breaks-up under 
small perturbation and only a few of its constituent invariant $m$-dimensional tori survive a perturbation.

The mathematical study of low-dimensional tori in Hamiltonian systems dates back to the late 1960s. The intensive treatment of the problem starts with pioneering papers [14] and [19]. We refer reader to the monographs [2], [4], [12], [15], 20] and the papers [9, [10, [21], and [24] for the state of art in the domain.

In this paper we deal with the problem of persistence of (n-1)- dimensional tori in setting close to the original Kolmogorov theorem. Our goal is to find conditions on the unperturbed Hamiltonian $H_{0}$ under which system (2.1) has an invariant torus of dimension $n-1$ for every analytic perturbation and all sufficiently small $\varepsilon$. This problem is still poor investigated. We refer to papers [5] and [8] for results and discussion.

We focus on the problem on persistence of hyperbolic invariant tori for arbitrary analytic perturbation of a Hamiltonian function. Throughout of the paper we assume that the frequency vector $\overline{\boldsymbol{\omega}}=\nabla_{p} H(0,0)$ has $n-1$ rationally independent components and the Hessian $H_{0}^{\prime \prime}(0,0)$ is nondegenerate. Then there exists, see [21], an affine symplectic transformation with rational coefficients such that in new variables

$$
\boldsymbol{p}=\left(\boldsymbol{y}, z_{2}\right) \in \mathbb{R}^{n-1} \times \mathbb{R}, \quad \boldsymbol{q}=\left(\boldsymbol{x}, z_{1}\right) \in \mathbb{T}^{n-1} \times \mathbb{T}, \quad \boldsymbol{z}=\left(z_{1}, z_{2}\right),
$$

the Hamiltonian $H(\boldsymbol{x}, \boldsymbol{y}, \boldsymbol{z})=H_{0}\left(\boldsymbol{y}, z_{1}\right)+H_{1}(\boldsymbol{x}, \boldsymbol{y}, \boldsymbol{z})$ satisfies the following condition.

H.1 Recall (1.2) and (1.3). There is $\varrho>0$ with the properties. The function $H_{0}\left(\boldsymbol{y}, z_{2}\right)$ is analytic in the complex ball $\left(\mathbf{y}, z_{2}\right) \in B_{3 \varrho}^{n}$; the perturbation $H_{1}(\boldsymbol{x}, \boldsymbol{y}, \boldsymbol{z})$ is analytic on the cartesian product of the complex strip $\left(\mathbf{x}, z_{1}\right) \in \Re_{3 \varrho}$ and the ball $\left(\mathbf{y}, z_{2}\right) \in B_{3 \varrho}^{n}$. In particular, $H_{1}$ is $2 \pi$-periodic in $\boldsymbol{x}$ and $z_{1}$. Moreover, there is $c>0$ such that

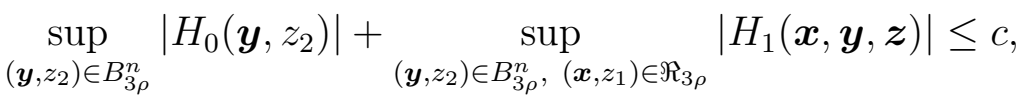

H.2 The frequency vector $\overline{\boldsymbol{\omega}}=(\boldsymbol{\omega}, 0)$ and Hamiltonian $H_{0}$ satisfy

$$
\nabla_{\boldsymbol{y}} H_{0}(0,0)=\boldsymbol{\omega} \in \mathbb{R}^{n-1}, \quad \partial_{z_{2}} H_{0}(0,0)=0 .
$$

The components of $\boldsymbol{\omega}$ are rationally independent. and satisfy the diophantine condition

$$
\left|\left(\boldsymbol{\omega}^{\top} \cdot \boldsymbol{s}\right)^{-1}\right| \leq c_{0}|\boldsymbol{s}|^{-n} \text { for all } \boldsymbol{s} \in \mathbb{Z}^{n-1} \backslash\{0\} .
$$


In the variables $(\mathbf{x}, \mathbf{y}, \mathbf{z})$ system (2.1) reads

$$
\dot{\boldsymbol{x}}=\nabla_{\boldsymbol{y}} H, \quad \dot{\boldsymbol{y}}=-\nabla_{\boldsymbol{x}} H, \quad \dot{\boldsymbol{z}}=\mathbf{J} \nabla_{\boldsymbol{z}} H, \quad \text { where } \mathbf{J}=\left(\begin{array}{cc}
0 & 1 \\
-1 & 0
\end{array}\right) .
$$

Definition 2.1. Hamiltonian system (2.6) has an analytic $(n-1)$-dimensional invariant torus with the frequency vector $\boldsymbol{\omega}$ if there exists an analytic canonical transform $\boldsymbol{\vartheta}:(\boldsymbol{\xi}, \boldsymbol{\eta}, \boldsymbol{\zeta}) \mapsto(\boldsymbol{x}, \boldsymbol{y}, \boldsymbol{z})$,

$$
\begin{array}{r}
\boldsymbol{x}=\boldsymbol{\xi}+\boldsymbol{u}(\boldsymbol{\xi}), \quad \boldsymbol{y}=\boldsymbol{v}(\boldsymbol{\xi})+O(|\boldsymbol{\eta}|,|\boldsymbol{\zeta}|), \\
\boldsymbol{\xi} \in \mathbb{T}^{n-1}, \quad \boldsymbol{\eta} \in \mathbb{R}^{n-1}, \quad \boldsymbol{\zeta} \in \mathbb{R}^{2},
\end{array}
$$

such that $\vartheta$ puts $H$ into the normal form, i.e.,

$$
N(\boldsymbol{\xi}, \boldsymbol{\eta}, \boldsymbol{\zeta}) \equiv H \circ \boldsymbol{\vartheta}=\boldsymbol{\omega} \cdot \boldsymbol{\eta}+\frac{1}{2} \boldsymbol{\Omega} \boldsymbol{\zeta} \cdot \boldsymbol{\zeta}+o\left(|\boldsymbol{\eta}|,|\boldsymbol{\zeta}|^{2}\right) .
$$

Here $\Omega$ is a constant symmetric matrix. Without loss of generality we may assume that

$$
\boldsymbol{\Omega}=\operatorname{diag}(-k, 1), \quad k \in \mathbb{R},
$$

and

$$
\overline{\mathbf{u}}:=(2 \pi)^{1-n} \int_{\mathbb{T}^{n-1}} \boldsymbol{u}(\boldsymbol{\xi}) d \boldsymbol{\xi}=0, \quad \overline{\boldsymbol{w}}=(\alpha, 0), \quad \alpha \in \mathbb{R} .
$$

The invariant torus is weakly-hyperbolic if $k \geq 0$. In the normal coordinates system (2.6) reads

$$
\dot{\boldsymbol{\xi}}=\boldsymbol{\omega}+O(|\boldsymbol{\eta}|,|\boldsymbol{\zeta}|), \quad \dot{\boldsymbol{\eta}}=O(|\boldsymbol{\eta}|,|\boldsymbol{\zeta}|), \quad \dot{\boldsymbol{\zeta}}=\mathbf{J} \boldsymbol{\Omega} \boldsymbol{\zeta}+O\left(|\boldsymbol{\eta}|,|\boldsymbol{\zeta}|^{2}\right) .
$$

It has the solution $\boldsymbol{\xi}=\boldsymbol{\omega} t+$ const, $\boldsymbol{\eta}=0, \boldsymbol{\zeta}=0$, which defines an invariant torus.

The invariant tori which meet all requirements of Definition 2.1 are known as reducible tori. It was proved in [3] that every analytic hyperbolic invariant torus is reducible.

If $\varepsilon=0$, then the canonical transformation $\vartheta$ is trivial

$$
\boldsymbol{x}=\boldsymbol{\xi}, \quad \boldsymbol{y}=\boldsymbol{\eta}, \quad \boldsymbol{z}=(\alpha, 0)+\boldsymbol{\zeta}
$$


and the normal form is given by

$$
\begin{aligned}
N\left(\boldsymbol{\eta}, \zeta_{2}\right) & \equiv H_{0}\left(\boldsymbol{\eta}, \zeta_{2}\right) \\
& =\boldsymbol{\omega} \cdot \boldsymbol{\eta}+\frac{1}{2} \zeta_{2}^{2}+\boldsymbol{t}_{0} \cdot \boldsymbol{\eta} \zeta_{2}+\frac{1}{2} \mathbf{S}_{0} \boldsymbol{\eta} \cdot \boldsymbol{\eta}+o\left(|\boldsymbol{\eta}|^{2},\left|\boldsymbol{\eta} \| \zeta_{2}\right|,\left|\zeta_{2}\right|^{2}\right)
\end{aligned}
$$

Here $\boldsymbol{t}_{0} \in \mathbb{R}^{n-1}$ and the Hessian $\mathbf{S}_{0}$ are defined by

$$
\boldsymbol{t}_{0}=\nabla_{y} \partial_{z_{2}} H(0,0), \quad \mathbf{S}_{0}=\partial_{y}^{2} H_{0}(0,0,0) .
$$

If $\varepsilon=0$, then $\Omega=\operatorname{diag}\{0,1\}$ is degenerate. In other words, the problem of persistence of lower dimensional tori is degenerate in the original Kolmogorov- Melnikov formulation. Introduce the constant matrix

$$
\mathbf{K}_{0}=\mathbf{S}_{0}-\boldsymbol{t}_{0} \otimes \boldsymbol{t}_{0}
$$

where the vector $\boldsymbol{t}_{0}$ and the matrix $\mathbf{S}_{0}$ are defined by equalities (2.11).

Theorem 2.2. Let Conditions (H.1) - (H.1) be satisfied. Furthermore, assume that

$$
\mathbf{K}_{0} \boldsymbol{\eta} \cdot \boldsymbol{\eta}<0 \quad \text { for all } \boldsymbol{\eta} \in \mathbb{R}^{n-1} \backslash\{0\} .
$$

Then there is $\varepsilon_{0}>0$ such that for all $\varepsilon \in\left(-\varepsilon_{0}, \varepsilon_{0}\right)$, Hamiltonian system (2.6) has an invariant $(n-1)$-dimensional weakly hyperbolic torus which meets all requirements of Definition 2.1.

Now we can characterize the contents of the paper. In this paper we apply to low-dimensional tori problem a modified version of the variational principle proposed in [16] and developed in [17]. In Section 3] we introduce the special group of canonical transforms which put the Hamiltonina $H$ in the normal form. We investigate in many details the structure of this group and its tangent space.

In the Section 4 we employ the version of the Lyapunov-Schmidt method developed in [19] in order to reduce the problem to the finite system of functional equations named bifurcation equations. To this end we add the modification term $m z_{1}+M z_{1}^{2} / 2$ to the original Hamiltonian. We obtain the modified Hamiltonian $H_{m}$,

$$
H_{m}(\mathbf{x}, \mathbf{y}, \mathbf{z})=H_{0}\left(\mathbf{y}, z_{2}\right)+\varepsilon H_{1}(\mathbf{x}, \mathbf{y}, \mathbf{z})+m z_{1}+\frac{1}{2} M z_{1}^{2}
$$


Thus we come to the following modified problem. For given $\alpha \in \mathbb{R}^{1}$ and $k \in[0,1]$ to find parameters $m, M$, and a canonical mapping

$$
\begin{aligned}
\boldsymbol{\theta}:(\boldsymbol{\xi}, \boldsymbol{\eta}, \boldsymbol{\zeta}) \rightarrow(\mathbf{x}, \mathbf{y}, \mathbf{z}), \\
\boldsymbol{x}=\boldsymbol{\xi}+\mathbf{u}(\boldsymbol{\xi}), \quad \mathbf{y}=\mathbf{v}+\mathbf{V}(\boldsymbol{\xi}) \boldsymbol{\eta}+\boldsymbol{\Lambda}(\boldsymbol{\xi}) \boldsymbol{\zeta}+\frac{1}{2} \boldsymbol{\zeta}^{\top} \mathbf{R}(\boldsymbol{\xi}) \boldsymbol{\zeta}, \\
\mathbf{z}=\mathbf{w}(\boldsymbol{\xi})+\mathbf{W}(\boldsymbol{\xi}) \boldsymbol{\zeta} .
\end{aligned}
$$

which puts the modified hamiltonian in the normal form ,i.e.,

$$
H_{m} \circ \boldsymbol{\theta}=\boldsymbol{\omega}^{\top} \cdot \boldsymbol{\eta}+\frac{1}{2} \boldsymbol{\zeta}^{\top} \boldsymbol{\Omega} \boldsymbol{\zeta}+o\left(|\boldsymbol{\eta}|,|\boldsymbol{\eta} \| \boldsymbol{\zeta}|,|\boldsymbol{\zeta}|^{2}\right),
$$

In this framework $\alpha$ and $k$ are given, while $m, M$, and $e$ are unknown and should be defined along with a solution. The solvability of the modified problem can be established by using the Nash-Moser implicit function theorem. The obtained solution to the modified problem is a function of $\alpha$ and $k$. In particular, we have $m=m(\alpha, k)$ and $M=M(\alpha, k)$. Obviously, if $m=M=0$, then the canonical mapping $\boldsymbol{\theta}$ puts the original hamiltonian $H(\mathbf{x}, \mathbf{y}, \mathbf{z})$ in the normal form. Thus we reduce the original problem to the system of two scalar equations

$$
m(\alpha, k)=M(\alpha, k)=0
$$

named the bifurcation equations.

In Sections 5-6 we prove that the modified problem has an analytic solution for all sufficiently small $\varepsilon$. In Sections 7.9 we investigate in details the dependence of solutions to the modified problem on the parameters $\alpha$ and $k$. We define the Jacobi vector fields associated with the derivatives of these solutions with respect to $\alpha$ and $k$. We also investigate the properties of the quadratic form of the differential of the action functional. Finally, in Section 10 we prove the existence of the critical point of the action functional and complete the proof of Theorem 2.2 .

\section{Basic group of of canonical transformations}

Recall that our task is to find the canonical transformation (2.7) which puts $H$ into the normal form (2.8). An essential tool in our approach is a special group of canonical transformations. In this section we define such a group as a manifold in the space of analytic mappings and investigate a structure of this manifold. 
Canonical transformations Let us consider the totality of all analytic mappings $\vartheta: \mathbb{T}^{n-1} \times \mathbb{R}^{n-1} \times \mathbb{R}^{2} \rightarrow \mathbb{T}^{n-1} \times \mathbb{R}^{n-1} \times \mathbb{R}^{2}$ of the form

$$
\begin{aligned}
& \boldsymbol{\vartheta}:(\boldsymbol{\xi}, \boldsymbol{\eta}, \boldsymbol{\zeta}) \rightarrow(\boldsymbol{x}, \boldsymbol{y}, \boldsymbol{z}), \\
& \boldsymbol{x}=\boldsymbol{\xi}+\mathbf{u}(\boldsymbol{\xi}), \quad \mathbf{y}=\mathbf{v}+\mathbf{V}(\boldsymbol{\xi}) \boldsymbol{\eta}+\boldsymbol{\Lambda}(\boldsymbol{\xi}) \boldsymbol{\zeta}+\frac{1}{2} \boldsymbol{\zeta}^{\top} \mathbf{R}(\boldsymbol{\xi}) \boldsymbol{\zeta} \\
& \mathbf{z}=\mathbf{w}(\boldsymbol{\xi})+\mathbf{W}(\boldsymbol{\xi}) \boldsymbol{\zeta} .
\end{aligned}
$$

Here $(n-1) \times(n-1)$ - matrix valued function $\mathbf{V},(n-1) \times 2$ - matrix valued function $\boldsymbol{\Lambda}, 2 \times 2$ - matrix values function $\mathbf{W}$, and vector valued functions $\mathbf{u}, \mathbf{v}: \mathbb{T}^{n-1} \rightarrow \mathbb{R}^{n-1}, \mathbf{v}: \mathbb{T}^{n-1} \rightarrow \mathbb{R}^{n-1}, \mathbf{w}: \mathbb{T}^{n-1} \rightarrow \mathbb{R}^{2}$, are analytic and $2 \pi$ periodic in $\boldsymbol{\xi}, \mathbf{R}$ is a vector valued quadratic form given by

$$
\boldsymbol{\zeta}^{\top} \mathbf{R} \boldsymbol{\zeta}=\left(\boldsymbol{\zeta}^{\top} \mathbf{R}_{1} \boldsymbol{\zeta}, \ldots, \boldsymbol{\zeta}^{\top} \mathbf{R}_{n-1} \boldsymbol{\zeta}\right)^{\top}, \quad \mathbf{R}_{i}=\mathbf{R}_{i}^{\top} .
$$

where $2 \times 2$ matrix valued functions $\mathbf{R}_{i}(\boldsymbol{\xi})$ are analytic and $2 \pi$ periodic.

Recall that $\boldsymbol{\vartheta}: \mathbb{T}^{n-1} \times \mathbb{R}^{n-1} \times \mathbb{R}^{2} \rightarrow \mathbb{T}^{n-1} \times \mathbb{R}^{n-1} \times \mathbb{R}^{2}$ is a canonical if $\boldsymbol{\vartheta}^{-1}$ takes solutions of hamiltonian system with a hamiltonian $H$ to solutions of the hamiltonian system with the hamiltonian $H \circ \boldsymbol{\vartheta}$. The mapping $\boldsymbol{\vartheta}$ is a canonical if and only if its Jacobi matrix is symplectic, i.e.,

$$
\left(\boldsymbol{\vartheta}^{\prime}\right)^{\top} \mathbf{J}_{2 n} \boldsymbol{\vartheta}^{\prime}=\mathbf{J}_{2 n},
$$

where

$$
\mathbf{J}_{2 n}=\left(\begin{array}{ccc}
0 & \mathbf{I} & 0 \\
-\mathbf{I}_{n-1} & 0 & 0 \\
0 & 0 & \mathbf{J}
\end{array}\right), \quad \mathbf{J}=\left(\begin{array}{cc}
0 & 1 \\
-1 & 0
\end{array}\right)
$$

I is the identity matrix. The following First Structure Theorem gives the complete description of the set of canonical mappings $\boldsymbol{\vartheta} \in \mathcal{A}$.

Theorem 3.1. First Structure Theorem. Let $\boldsymbol{\vartheta} \in \mathcal{A}$ is given by (3.1). Furthermore assume that the there are analytic inverses $(I d+\mathbf{u})^{-1}, \mathbf{V}^{-1}$, and $\mathbf{W}^{-1}$. Then $\boldsymbol{\vartheta}$ is canonical if and only if

$$
\begin{gathered}
\mathbf{V}=\left(\mathbf{I}_{n-1}+\mathbf{u}^{\prime}\right)^{-\top} \\
\operatorname{det} \mathbf{W}=1 \Leftrightarrow \mathbf{W}^{\top} \mathbf{J} \mathbf{W}=\mathbf{J} \\
\mathbf{\Lambda}=-\mathbf{V}\left(\mathbf{w}^{\prime}\right)^{\top} \mathbf{J} \mathbf{W} \equiv-\mathbf{V} \nabla \mathbf{w} \mathbf{J} \mathbf{W}, \\
\mathbf{R}_{i}=-V_{i k} \frac{\partial}{\partial \xi_{k}}\left(\mathbf{W}^{\top}\right) \mathbf{J} \mathbf{W}, \\
d\left(\xi_{k}+u_{k}\right) \wedge d v_{k}+d w_{1} \wedge d w_{2}=0 .
\end{gathered}
$$


Moreover, there exist $\beta \in \mathbb{R}^{n-1}$ and $2 \pi$-periodic scalar function $\varphi_{0}(\boldsymbol{\xi})$ such that

$$
\mathbf{v}=\beta+\mathbf{V}\left(\nabla \varphi_{0}-w_{2} \nabla w_{1}\right) .
$$

Proof. The proof is given in Appendix A.

It is clear that the totality of the canonical mappings $\boldsymbol{\vartheta}$ contains the identity mapping and it is closed with respect to the composition. In other words, it can be regarded as a subgroup of the group of analytical diffeomorphisms. Every mapping $\boldsymbol{\vartheta}$ is completely characterized by the vector $\boldsymbol{\Theta}$ of its coefficients,

$$
\Theta=\left(\mathbf{u}, \mathbf{v}, \mathbf{w}, \mathbf{V}, \Lambda, \mathbf{W}, \mathbf{R}_{i}\right) .
$$

The group structure on the set of the canonical mappings $\boldsymbol{\vartheta}$ induces the group structure on the set of the corresponding vectors $\boldsymbol{\Theta}$. Hence the totality of coefficient vectors $\Theta$ corresponding to the canonical transforms $\boldsymbol{\vartheta}$ can be regarded as a nonlinear manifold in the linear space of all vector-valued analytic $2 \pi$-periodic vector-valued functions $\boldsymbol{\Theta}$. We denote this manifold by $\mathcal{G}$. Now our task is to supply $\mathcal{G}$ with a local chart.

In view of Theorem 3.1 the vector $\Theta$ are completely defined by a constant vector $\beta$, a scalar function $\varphi_{0}$, vector functions $\mathbf{u}, \mathbf{w}$, and by three elements of a symplectic matrix $\mathbf{W}$. We can consider these quantities as local coordinates for the manifold $\mathcal{G}$. More precisely, introduce the vector function

$$
\boldsymbol{\varphi}=\left(\beta, \varphi_{0}, \mathbf{u}, \mathbf{w}, W_{11}, W_{12}, W_{21}\right)
$$

such that the functions $W_{11}, W_{12}, W_{21}, \varphi_{0}: \mathbb{T}^{n-1} \rightarrow \mathbb{R}$, the vector functions $\mathbf{u}: \mathbb{T}^{n-1} \rightarrow \mathbb{R}^{n-1}, \mathbf{w}: \mathbb{T}^{n-1} \rightarrow \mathbb{R}^{2}$ are analytic and

$$
\overline{\mathbf{u}} \equiv(2 \pi)^{1-n} \int_{\mathbb{T}^{n-1}} \mathbf{u} d \xi=0, \quad \overline{\psi_{0}} \equiv(2 \pi)^{1-n} \int_{\mathbb{T}^{n-1}} \psi_{0} d \xi=0,
$$

Next, introduce the mapping $\boldsymbol{\Theta}(\boldsymbol{\varphi})$ defined by the formulae

$$
\Theta: \varphi \mapsto \Theta(\varphi)=\left(\mathbf{u}, \mathbf{v}, \mathbf{w}, \mathbf{V}, \boldsymbol{\Lambda}, \mathbf{W}, \mathbf{R}_{i}\right),
$$

where

$$
\begin{array}{r}
\mathbf{V}=\left(\mathbf{I}_{n-1}+\mathbf{u}^{\prime}\right)^{-\top}, \quad \mathbf{v}=\beta+\mathbf{V}\left(\nabla \varphi_{0}-w_{2} \nabla w_{1}\right), \\
\mathbf{W}=\left(\begin{array}{cc}
W_{11} & W_{12} \\
W_{21} & \frac{1}{W_{11}}\left(1+W_{12} W_{21}\right)
\end{array}\right), \\
\boldsymbol{\Lambda}=-\mathbf{V}\left(\mathbf{w}^{\prime}\right)^{\top} \mathbf{J} \mathbf{W}, \quad \mathbf{R}_{i}=-V_{i k}\left(\frac{\partial}{\partial \xi_{k}} \mathbf{W}^{\top}\right) \mathbf{J} \mathbf{W} .
\end{array}
$$


In view of Theorem 3.1 the mapping $\varphi \mapsto \Theta(\varphi)$ takes the vectors $\boldsymbol{\varphi}$ to the elements of the manifold $\mathcal{G}$. Hence, this mapping defines a local chart on $\mathcal{G}$. This chart is local since the mapping (3.7) develops singularities at the points where $W_{11}=0$ and $\operatorname{det}\left(\mathbf{I}_{n-1}+\mathbf{u}^{\prime}\right)=0$.

Tangent space. Denote by $\mathcal{L}$ the linear space of all coordinate vectors $\boldsymbol{\varphi}$. Introduce that Gateaux differential

$$
D \boldsymbol{\Theta}(\boldsymbol{\varphi})[\delta \boldsymbol{\varphi}]=\lim _{t \rightarrow 0} t^{-1}(\boldsymbol{\Theta}(\boldsymbol{\varphi}+t \delta \boldsymbol{\varphi})-\boldsymbol{\Theta}(\boldsymbol{\varphi}))
$$

The totality of all vectors

$$
\delta \boldsymbol{\Theta}=D \boldsymbol{\Theta}(\boldsymbol{\varphi})[\delta \boldsymbol{\varphi}], \quad \delta \boldsymbol{\varphi} \in \mathcal{L},
$$

can be regarded as the tangential space $\operatorname{Tan}_{\Theta} \mathcal{G}$ to the manifold $\mathcal{G}$ at the point $\Theta(\varphi)$. Direct calculations show that for every

$$
\delta \boldsymbol{\varphi}=\left(\delta \beta, \delta \varphi_{0}, \delta \mathbf{u}, \delta \mathbf{w}, \delta W_{11}, \delta W_{12}, \delta W_{21}\right)
$$

the vector

$$
\delta \boldsymbol{\Theta}=\left(\delta \mathbf{u}, \delta \mathbf{v}, \delta \mathbf{w}, \delta \mathbf{V}, \delta \mathbf{W}, \delta \mathbf{\Lambda}, \delta \mathbf{R}_{i}\right) .
$$

is defined by the following formulae

$$
\begin{gathered}
\delta \mathbf{V}=-\mathbf{V} \delta \nabla_{\xi} \delta \mathbf{u} \mathbf{V}, \quad \delta W_{22}=\frac{1}{W_{11}}\left(W_{12} \delta W_{21}+W_{21} \delta W_{12}-W_{22} \delta W_{11}\right), \\
\delta \mathbf{W}=\left(\begin{array}{cc}
\delta W_{11} & \delta W_{12} \\
\delta W_{21} & \delta W_{22}
\end{array}\right), \\
\delta \mathbf{v}=\delta \beta+\mathbf{V}\left(\nabla \delta \varphi_{0}-w_{2} \nabla \delta w_{1}-\delta w_{2} \nabla w_{1}\right)+\delta \mathbf{V}\left(\nabla \varphi_{0}-w_{2} \nabla w_{1}\right), \\
\delta \boldsymbol{\Lambda}=\delta \mathbf{V} \nabla w \mathbf{J} \mathbf{W}-\mathbf{V} \nabla(\delta \mathbf{W}) \mathbf{J} \mathbf{W}-\mathbf{W} \nabla \mathbf{w} \mathbf{J} \delta \mathbf{W} \\
\delta \mathbf{R}_{i}=-\delta V_{i k} \frac{\partial}{\partial \xi_{k}}\left(\mathbf{W}^{\top}\right) \mathbf{J W}-V_{i k} \frac{\partial}{\partial \xi_{k}}\left(\delta \mathbf{W}^{\top}\right) \mathbf{J} \mathbf{W}-V_{i k} \frac{\partial}{\partial \xi_{k}}\left(\mathbf{W}^{\top}\right) \mathbf{J} \delta \mathbf{W}
\end{gathered}
$$

The right hand sides of (3.13) are linear differential operators acting on $\delta \boldsymbol{\varphi}$. They can be regarded as the Gâteaux derivatives of the components of $\Theta(\varphi)$ at the point $\boldsymbol{\varphi}$ in the direction $\delta \boldsymbol{\varphi}$. These relations can be simplified in the particular case when

$$
\boldsymbol{\varphi}=\boldsymbol{\varphi}_{0}(\alpha) \equiv\left(0,0,0, \alpha \mathbf{e}_{1}, 1,0,0\right)
$$


Notice that for $\varphi=\varphi_{0}$ we have

$$
\mathbf{V}=\mathbf{I}_{n-1}, \quad \mathbf{W}=\mathbf{I}, \quad \boldsymbol{\Lambda}=0, \quad \mathbf{R}=0 .
$$

We consider this very special case in many details. For $\boldsymbol{\varphi}=\boldsymbol{\varphi}_{0}$ we will use the special notation for the components of the vector $\delta \boldsymbol{\varphi}$ :

$$
\boldsymbol{\Upsilon} \equiv \delta \boldsymbol{\varphi}=\left(\boldsymbol{\nu}, \psi_{0}, \boldsymbol{\chi}, \boldsymbol{\lambda}, \Gamma_{11}, \Gamma_{12}, \Gamma_{21}\right)
$$

This means that

$$
\begin{gathered}
\boldsymbol{\nu}=\delta \boldsymbol{\beta}, \quad \psi_{0}=\delta \varphi_{0}, \quad \chi=\delta \boldsymbol{u}, \quad \boldsymbol{\lambda}=\delta \mathbf{w} \\
\Gamma_{11}=\delta W_{11}, \quad \Gamma_{12}=\delta W_{12}, \quad \Gamma_{21}=\delta W_{21}
\end{gathered}
$$

It is easily seen that in this case

$$
\mathfrak{Z}:=\delta \boldsymbol{\Theta} \equiv D_{\varphi} \boldsymbol{\Theta}\left(\boldsymbol{\varphi}_{0}\right)[\Upsilon \boldsymbol{\Upsilon}]
$$

is defined by the equalities

$$
\mathfrak{Z} \equiv\left(\chi, \boldsymbol{\mu}, \boldsymbol{\lambda},-\nabla \boldsymbol{\chi}, \boldsymbol{\Gamma}, \nabla(\mathbf{J} \boldsymbol{\lambda}), \partial_{\xi_{i}}(\mathbf{J} \boldsymbol{\Gamma})\right),
$$

where

$$
\boldsymbol{\mu}=\boldsymbol{\nu}+\nabla \psi_{0}, \quad \operatorname{Tr} \boldsymbol{\Gamma}=0 .
$$

This means that in this case

$$
\begin{gathered}
\delta \mathbf{V}=-\nabla_{\xi} \boldsymbol{\chi}, \quad \delta \boldsymbol{\Lambda}=-\nabla \boldsymbol{\lambda} \mathbf{J} \equiv \nabla(\mathbf{J} \boldsymbol{\lambda}), \quad \mathbf{R}_{i}=-\frac{\partial}{\partial \xi_{i}} \boldsymbol{\Gamma}^{\top} \mathbf{J} \equiv \frac{\partial}{\partial \xi_{i}}(\mathbf{J} \boldsymbol{\Gamma}) . \\
\delta \mathbf{W}=\boldsymbol{\Gamma}=\left(\begin{array}{ll}
\Gamma_{11} & \Gamma_{12} \\
\Gamma_{21} & \Gamma_{22}
\end{array}\right), \text { where } \Gamma_{22}=-\Gamma_{11} .
\end{gathered}
$$

In other words, we have the equality $\operatorname{Tan} \Theta\left(\boldsymbol{\varphi}_{0}\right) \mathcal{G}=\{\mathfrak{Z}\}$. In particular, all tangent spaces to the manifold $\mathcal{G}$ at points $\Theta\left(\boldsymbol{\varphi}_{0}\right)$ coincide. It is a remarkable fact of the theory that for every $\varphi$ there is a canonical algebraic isomorphism between the tangent space to the manifold $\mathcal{G}$ at point $\Theta(\varphi)$ and the tangent space to $\mathcal{G}$ at $\Theta\left(\boldsymbol{\varphi}_{0}\right)$. This result is given by the following theorem

Theorem 3.2. Second Structure Theorem. (i) Let $\varphi \in \mathcal{L}$. Let $\Upsilon$ and $\mathfrak{Z}$ be given by (3.14) and (3.17). Then there exists a vector field

$$
\delta \boldsymbol{\varphi}=\left(\delta \beta, \delta \varphi_{0}, \delta \mathbf{u}, \delta \mathbf{w}, \delta W_{11}, \delta W_{12}, \delta W_{21}\right) \in X_{\sigma, d-1}
$$


such that the corresponding vector of coefficients

$$
\delta \boldsymbol{\Theta}(\boldsymbol{\varphi})=\left(\delta \mathbf{u}, \delta \mathbf{v}, \delta \mathbf{w}, \delta \mathbf{V}, \delta \mathbf{W}, \delta \boldsymbol{\Lambda}, \delta \mathbf{R}_{i}\right)
$$

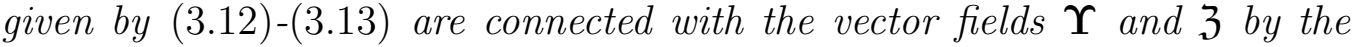
relations

$$
\begin{gathered}
\delta \mathbf{u}=\chi_{i} \frac{\partial}{\partial \xi_{i}}(\boldsymbol{\xi}+\mathbf{u}), \\
\delta \mathbf{v}=\mathbf{V} \boldsymbol{\mu}+\boldsymbol{\Lambda} \boldsymbol{\lambda}+\chi_{i} \frac{\partial}{\partial \xi_{i}} \mathbf{v}, \\
\delta \mathbf{W}=\mathbf{W} \boldsymbol{\lambda}+\chi_{i} \frac{\partial}{\partial \xi_{i}} \mathbf{w}, \\
\delta \mathbf{W}=\mathbf{W} \boldsymbol{\Gamma}+\chi_{i} \frac{\partial}{\partial \xi_{i}} \mathbf{W}, \\
\delta \mathbf{V}=-\mathbf{V} \nabla_{\xi} \boldsymbol{\chi}+\chi_{i} \frac{\partial}{\partial \xi_{i}} \mathbf{V}, \\
\delta \boldsymbol{\Lambda}=\mathbf{V} \nabla_{\xi}(\mathbf{J} \boldsymbol{\lambda})+\chi_{i} \frac{\partial}{\partial \xi_{i}} \boldsymbol{\Lambda}+\boldsymbol{\lambda}^{\top} \mathbf{R}+\boldsymbol{\Lambda} \boldsymbol{\Gamma}, \\
\delta \mathbf{R}_{i}=\frac{\partial}{\partial \xi_{i}}(\mathbf{J} \boldsymbol{\Gamma})+\mathbf{R}_{i} \boldsymbol{\Gamma}+\left(\mathbf{R}_{i} \boldsymbol{\Gamma}\right)^{\top}+\chi_{i} \frac{\partial}{\partial \xi_{i}} \mathbf{R}_{i}, \\
\delta \boldsymbol{\beta}=\boldsymbol{\nu}, \quad \delta \varphi_{0}=\psi_{0}+w_{2} \delta w_{1}+\chi_{i} \frac{\partial}{\partial \xi_{i}} \varphi_{0}-w_{2} \chi_{i} \frac{\partial}{\partial \xi_{i}} w_{1}-\boldsymbol{\nu} \cdot \mathbf{u} .
\end{gathered}
$$

Here $\mathbf{u}, \varphi_{0}, \mathbf{W}, \mathbf{V}, \boldsymbol{\Lambda}$, and $\mathbf{R}_{i}$ are the components of the vector fields $\boldsymbol{\varphi}$ and $\Theta(\varphi)$.

(ii) Conversely, let $\delta \boldsymbol{\varphi}$ and $\delta \boldsymbol{\Theta}(\boldsymbol{\varphi})$ be given by the relations (3.11)-(3.13) . Then there exist vector fields

$$
\Upsilon=\left(\boldsymbol{\nu}, \psi_{0}, \boldsymbol{\chi}, \boldsymbol{\lambda}, \Gamma_{11}, \Gamma_{12}, \Gamma_{21}\right) \in X_{\sigma, d-1}
$$

and

$$
\mathfrak{Z} \equiv\left(\boldsymbol{\chi}, \boldsymbol{\mu}, \boldsymbol{\lambda},-\nabla \boldsymbol{\chi}, \boldsymbol{\Gamma}, \nabla(\mathbf{J} \boldsymbol{\lambda}), \partial_{\xi_{i}}(\mathbf{J} \boldsymbol{\Gamma})\right),
$$

which are connected with the components of the vector fields $\delta \boldsymbol{\varphi}$ and $\delta \boldsymbol{\Theta}$ by 
the recurrent relations

$$
\begin{gathered}
\boldsymbol{\chi}=\mathbf{V}^{\top} \delta \mathbf{u} \\
\boldsymbol{\lambda}=\mathbf{W}^{-1} \delta \mathbf{W}-\chi_{i} \mathbf{W}^{-1} \frac{\partial}{\partial \xi_{i}} \mathbf{W} \\
\boldsymbol{\Gamma}=\mathbf{W}^{-1} \delta \mathbf{W}-\chi_{i} \mathbf{W}^{-1} \frac{\partial}{\partial \xi_{i}} \mathbf{W} \\
\boldsymbol{\mu}=\mathbf{V}^{-1}\left(\delta \mathbf{W}+\chi_{i} \frac{\partial}{\partial \xi_{i}} \mathbf{v}-\boldsymbol{\Lambda} \boldsymbol{\lambda}\right) \\
\nabla \psi_{0}=\boldsymbol{\mu}-\boldsymbol{\nu}, \quad \boldsymbol{\nu}=\frac{1}{(2 \pi)^{n-1}} \int_{\mathbb{T}^{n-1}} \boldsymbol{\mu} d \boldsymbol{\xi}=\delta \boldsymbol{\beta} .
\end{gathered}
$$

Proof. The proof is in Appendix B.

\section{A modified problem and bifurcation equa- tions}

Recall that the main problem is to prove that the Hamiltonian $H(\mathbf{x}, \mathbf{y}, \mathbf{z})=$ $H_{0}\left(\mathbf{y}, z_{2}\right)+\varepsilon H_{1}(\mathbf{x}, \mathbf{y}, \mathbf{z})$ has $(n-1)$-dimensional weakly-hyperbolic invariant torus for all sufficiently small $\varepsilon$. In view of Definition 2.1 it is necessary to find a canonical mapping $\boldsymbol{\theta}:(\boldsymbol{\xi}, \boldsymbol{\eta}, \boldsymbol{\zeta}) \rightarrow(\mathbf{x}, \mathbf{y}, \mathbf{z})$ which puts $H$ in the normal form

$$
H \circ \boldsymbol{\theta}=e+\boldsymbol{\omega}^{\top} \cdot \boldsymbol{\eta}+\frac{1}{2} \boldsymbol{\zeta}^{\top} \boldsymbol{\Omega} \boldsymbol{\zeta}+o\left(|\boldsymbol{\eta}|,|\boldsymbol{\eta} \| \boldsymbol{\zeta}|,|\boldsymbol{\zeta}|^{2}\right),
$$

where $\boldsymbol{\Omega}=\operatorname{diag}(-k, 1), k \in[0,1]$. and $e=$ const . For $\varepsilon=0$ the problem has a family of solutions given by

$$
\mathbf{x}=\boldsymbol{\xi}, \quad \mathbf{y}=\boldsymbol{\eta}, \quad \mathbf{z}=\boldsymbol{\alpha}+\boldsymbol{\zeta}, \quad \boldsymbol{\alpha}=(\alpha, 0)^{\top}, \quad e=k=0, \quad \alpha \in \mathbb{R}^{1} .
$$

It is easily seen that $(n-1)$ - dimensional manifold $\mathbb{T}_{\alpha}=\{\mathbf{x}=\boldsymbol{\xi}, \mathbf{y}=0, \mathbf{z}=$ $\boldsymbol{\alpha}\}$ is an invariant torus of the unperturbed system. The totality of these tori forms a foliation of resonance $n$-dimensional invariant torus, and $\alpha$ can be considered as a label of a leave of this foliation. Notice that $\alpha$ is nothing else but the mean value of $z_{1}$ over the invariant torus $\mathbb{T}_{\alpha}$. Perturbations destroy $n$-dimensional resonance torus, and only a few $(n-1)$-dimensional invariant tori survive for $\varepsilon \neq 0$. The label $\alpha$ of surviving torus is unknown and should be defined along with a solution to the problem. Therefore, the 
range of unknowns $(\alpha, k)$ is the whole strip $\mathbb{R} \times[0,1]$. This means that the problem of finding of $\alpha$ and $k$ is not local and can not be solved by using an iteration process. In order to cope with this difficulty we apply the version of the Lyapunov-Schmidt method proposed in [19]. Following [19] we add the modification term $m z_{1}+M z_{1}^{2} / 2$ to the original Hamiltonian. Thus we get the modified Hamiltonian $H$

$$
H_{m}(\mathbf{x}, \mathbf{y}, \mathbf{z})=H_{0}\left(\mathbf{y}, z_{2}\right)+\varepsilon H_{1}(\mathbf{x}, \mathbf{y}, \mathbf{z})+\mathbf{m}^{\top} \cdot \mathbf{z}+\frac{1}{2} \mathbf{z}^{\top} \mathbf{M} \mathbf{z}
$$

where

$$
\mathbf{m}=(m, 0)^{\top}, \quad \mathbf{M}=\operatorname{diag}(M, 0)
$$

Consider the following

Modified problem. For given $\alpha \in \mathbb{R}^{1}$ and $k \in[0,1]$ to find parameters $m, M, e$, and a canonical mapping

$$
\begin{aligned}
\boldsymbol{\theta}:(\boldsymbol{\xi}, \boldsymbol{\eta}, \boldsymbol{\zeta}) \rightarrow(\mathbf{x}, \mathbf{y}, \mathbf{z}), \\
\boldsymbol{x}=\boldsymbol{\xi}+\mathbf{u}(\boldsymbol{\xi}), \quad \mathbf{y}=\mathbf{v}+\mathbf{V}(\boldsymbol{\xi}) \boldsymbol{\eta}+\boldsymbol{\Lambda}(\boldsymbol{\xi}) \boldsymbol{\zeta}+\frac{1}{2} \boldsymbol{\zeta}^{\top} \mathbf{R}(\boldsymbol{\xi}) \boldsymbol{\zeta} \\
\mathbf{z}=\mathbf{w}(\boldsymbol{\xi})+\mathbf{W}(\boldsymbol{\xi}) \boldsymbol{\zeta}
\end{aligned}
$$

with the following properties. The mapping $\boldsymbol{\theta}$ satisfies the condition

$$
\frac{1}{(2 \pi)^{n-1}} \int_{\mathbb{T}^{n-1}} w_{1}(\xi) d \xi=\alpha
$$

and puts the modified hamiltonian in the normal form, i.e.,

$$
H_{m} \circ \boldsymbol{\theta}=e+\boldsymbol{\omega}^{\top} \cdot \boldsymbol{\eta}+\frac{1}{2} \boldsymbol{\zeta}^{\top} \boldsymbol{\Omega} \boldsymbol{\zeta}+o\left(|\boldsymbol{\eta}|,|\boldsymbol{\eta}||\boldsymbol{\zeta}|,|\boldsymbol{\zeta}|^{2}\right),
$$

We stress that in this framework $\alpha$ and $k$ are given, while $m, M$, and $e$ are unknown and should be defined along with a solution. The advantage of this approach is that the solvability of the modified problem can be established by using the Nash-Moser implicit function theorem. The obtained solution $\boldsymbol{\theta}, e, m, M$ to the modified problem is a function of $\alpha$ and $k$. In particular, we have $m=m(\alpha, k)$ and $M=M(\alpha, k)$. Obviously, if $m=M=0$, then the canonical mapping $\boldsymbol{\theta}$ puts the original hamiltonian $H(\mathbf{x}, \mathbf{y}, \mathbf{z})$ in the normal form. Thus we reduce the original problem to the system of two scalar equations

$$
m(\alpha, k)=M(\alpha, k)=0
$$


named the bifurcation equations. Hence our first task is to prove the local existence and uniqueness of solutions to the modified problem for all $(\alpha, k)$ and for all sufficiently small $\varepsilon$.

We reduce the modified problem to the system of nonlinear partial differential equations. Notice that in view of Theorem 3.1 the coefficient vectors $\boldsymbol{\Theta}=\left(\mathbf{u}, \mathbf{v}, \mathbf{w}, \mathbf{V}, \boldsymbol{\Lambda}, \mathbf{W}, \mathbf{R}_{i}\right)$ is a function of the coordinate vector

$$
\boldsymbol{\varphi}=\left(\beta, \varphi_{0}, \mathbf{u}, \mathbf{w}, W_{11}, W_{12}, W_{21}\right)
$$

Moreover, the mapping $\varphi \mapsto \Theta(\varphi)$ is given by explicit formulae (3.4). Thus relation (4.6) can be regarded as a system of nonlinear equations for $\boldsymbol{\varphi}$. Substituting representation (4.5) with the coefficients $\Theta=\Theta(\varphi)$ into (4.6) we arrive at the following system of equations for the vector $\varphi$ given by (4.8).

$$
\begin{gathered}
\Phi_{1}(\boldsymbol{\varphi}, e, m, M) \equiv H_{m}(\mathrm{id}+\mathbf{u}, \mathbf{v}, \mathbf{w})-e=0 \\
\Phi_{2}(\boldsymbol{\varphi}, m, M) \equiv\left\{\frac{\partial H_{m}}{\partial \mathbf{y}}(\mathrm{id}+\mathbf{u}, \mathbf{v}, \mathbf{w}) \boldsymbol{\Lambda}+\frac{\partial H_{m}}{\partial \mathbf{z}}(\mathrm{id}+\mathbf{u}, \mathbf{v}, \mathbf{w}) \mathbf{W}\right\}^{\top}=0 \\
\Phi_{3}(\boldsymbol{\varphi}, m, M) \equiv\left\{\frac{\partial H_{m}}{\partial \mathbf{y}}(\mathrm{id}+\mathbf{u}, \mathbf{v}, \mathbf{w}) \mathbf{V}\right\}^{\top}-\mathbf{\omega}=0 \\
\Phi_{4}(\boldsymbol{\varphi}, m, M) \equiv \frac{\partial H_{m}}{\partial y_{i}}(\mathrm{id}+\mathbf{u}, \mathbf{v}, \mathbf{w}) \mathbf{R}_{i}+\boldsymbol{\Lambda}^{\top} \frac{\partial^{2} H_{m}}{\partial \mathbf{y}^{2}}(\mathrm{id}+\mathbf{u}, \mathbf{v}, \mathbf{w}) \boldsymbol{\Lambda}+ \\
\mathbf{W}^{\top} \frac{\partial^{2} H_{m}}{\partial \mathbf{z} \partial \mathbf{y}}(\mathrm{id}+\mathbf{u}, \mathbf{v}, \mathbf{w}) \boldsymbol{\Lambda}+\left(\mathbf{W}^{\top} \frac{\partial^{2} H_{m}}{\partial \mathbf{z} \partial \mathbf{y}}(\mathrm{id}+\mathbf{u}, \mathbf{v}, \mathbf{w}) \boldsymbol{\Lambda}\right)^{\top}+ \\
\Phi_{5}(\boldsymbol{\varphi}) \equiv \frac{1}{(2 \pi)^{n-1}} \int_{\mathbb{T}^{n-1}} w_{1}(\xi) d \xi-\alpha=0 .
\end{gathered}
$$

Here matrices $\partial^{2} H_{m} /(\partial \mathbf{y})^{2}, \partial^{2} H_{m} /(\partial \mathbf{z} \partial \mathbf{y})$, and $\partial^{2} H_{m} /(\partial \mathbf{z})^{2}$ have the entries

$$
\begin{gathered}
\left(\frac{\partial^{2} H_{m}}{\partial \mathbf{y}^{2}}\right)_{i j}=\frac{\partial^{2} H_{m}}{\partial y_{i} \partial y_{j}}, \quad\left(\frac{\partial H_{m}}{\partial \mathbf{z} \partial \mathbf{y}}\right)_{p j}=\frac{\partial^{2} H_{m}}{\partial z_{p} \partial y_{j}}, \quad\left(\frac{\partial H_{m}}{\partial \mathbf{z}^{2}}\right)_{p q}=\frac{\partial H_{m}}{\partial z_{p} \partial z_{q}} \\
1 \leq i, j \leq n-1, \quad 1 \leq p, q \leq 2
\end{gathered}
$$


the row vectors $\partial H_{m} / \partial \mathbf{y}, \partial H_{m} / \partial \mathbf{z}$ are given by (1.1), i.e.,

$$
\frac{\partial H_{m}}{\partial \mathbf{y}}=\left(\nabla_{y} H_{m}\right)^{\top}, \quad \frac{\partial H_{m}}{\partial \mathbf{z}}=\left(\nabla_{z} H_{m}\right)^{\top}
$$

The matrices $\boldsymbol{\Lambda}, \mathbf{V}, \mathbf{W}$ and the vector $\mathbf{v}$ are expressed in terms of $\boldsymbol{\varphi}$ by equalities (3.8):

$$
\begin{array}{r}
\mathbf{V}=\left(\mathbf{I}_{n-1}+\mathbf{u}^{\prime}\right)^{-\top}, \quad \mathbf{v}=\beta+\mathbf{V}\left(\nabla \varphi_{0}-w_{2} \nabla w_{1}\right), \\
\mathbf{W}=\left(\begin{array}{cc}
W_{11} & W_{12} \\
W_{21} & \frac{1}{W_{11}}\left(1+W_{12} W_{21}\right)
\end{array}\right), \\
\mathbf{\Lambda}=-\mathbf{V}\left(\mathbf{w}^{\prime}\right)^{\top} \mathbf{J} \mathbf{W}, \quad \mathbf{R}_{i}=-V_{i k}\left(\frac{\partial}{\partial \xi_{k}} \mathbf{W}^{\top}\right) \mathbf{J} \mathbf{W} .
\end{array}
$$

Notice that the matrices $\mathbf{R}_{i}$ are symmetric and the operator $\Phi_{4}$ is a symmetric matrix valued function. Hence $\Phi_{1}$ is a scalar, $\Phi_{2}$ is a column vector of dimension $2, \Phi_{3}$ is a column vector of dimension $n-1, \Phi_{4}$ is $2 \times 2$ symmetric matrix. Therefore, equations (4.9a)-(4.9d) form a system of $(n+5)$ nonlinear differential equations for $n+5$ functional components of the vector- valued function $\varphi$.

Notice that a solution to the modified problem is not unique. It is easily seen that if a canonical mapping $(\boldsymbol{\xi}, \boldsymbol{\eta}, \boldsymbol{\zeta}) \rightarrow(\mathbf{x}, \mathbf{y}, \mathbf{z})$ puts the hamiltonian $H_{m}$ to the normal form, then the mapping

$$
\begin{gathered}
\left(\boldsymbol{\xi}^{\prime}, \boldsymbol{\eta}^{\prime}, \boldsymbol{\zeta}^{\prime}\right) \rightarrow(\boldsymbol{\xi}, \boldsymbol{\eta}, \boldsymbol{\zeta}) \rightarrow(\mathbf{x}, \mathbf{y}, \mathbf{z}), \\
\boldsymbol{\xi}=\boldsymbol{\xi}^{\prime}+c, \quad \boldsymbol{\eta}=\boldsymbol{\eta}^{\prime}, \quad \boldsymbol{\zeta}=\left(\begin{array}{cc}
\left(1+k b^{2}\right)^{1 / 2} & b \\
k b & \left(1+k b^{2}\right)^{1 / 2}
\end{array}\right) \boldsymbol{\zeta}^{\prime},
\end{gathered}
$$

$c \in \mathbb{R}^{n-1}, b \in \mathbb{R}$, also puts $H_{m}$ in the normal form. Hence the modified problem has $n$-parametric family of solutions. In order to eliminate this arbitrariness, we impose the orthogonality conditions

$$
\int_{\mathbb{T}^{n-1}} \mathbf{u}(\boldsymbol{\xi}) d \xi=0, \quad \int_{\mathbb{T}^{n-1}} W_{12}(\boldsymbol{\xi}) d \xi=0 .
$$

Recall that by definition of the local coordinate on the manifold $\mathcal{G}$, the function $\varphi_{0}$ in (4.8) has zero mean. Thus we have to add the orthogonality condition

$$
\int_{\mathbb{T}^{n-1}} \varphi_{0}(\boldsymbol{\xi}) d \xi=0
$$


Equations (4.9a)-(4.9e) and conditions 4.9g)-(4.9h) form a closed system of equations for $\varphi$ and parameters $m, M, e$.

Throughout of the paper we will use the following consequence of this equations. Introduce the important differential operator

$$
\boldsymbol{\partial}:=\boldsymbol{\omega}^{\top} \cdot \nabla_{\xi} \equiv \omega_{i} \frac{\partial}{\partial \xi_{i}} .
$$

Lemma 4.1. If $\boldsymbol{\varphi}$ is an analytic solution to equations (4.9a)- (4.9d) and $\mathbf{v}$ is given by (4.9f), then

$$
\begin{array}{r}
\boldsymbol{\omega}+\boldsymbol{\partial} \mathbf{u}=\nabla_{y} H_{m}(\boldsymbol{\xi}+\mathbf{u}, \mathbf{v}, \mathbf{w}), \\
\boldsymbol{\partial} \mathbf{v}=-\nabla_{x} H_{m}(\boldsymbol{\xi}+\mathbf{u}, \mathbf{v}, \mathbf{w}), \quad \boldsymbol{\partial} \mathbf{w}=\mathbf{J} \nabla_{z} H_{m}(\boldsymbol{\xi}+\mathbf{u}, \mathbf{v}, \mathbf{w}) .
\end{array}
$$

The proof is in Appendix C.

Operator equation. Now we reduce system of differential equations (4.9) to the operator equation in Banach spaces of analytic functions. Notice that unknowns are the vector-valued function $\varphi$ and the constants $e, m$, and $M$. Equations (4.9) also depend on the parameters $\alpha$ and $k$. It is convenient to introduce the vectors

$$
\mathfrak{f}=(\alpha, k), \quad \mathfrak{u}=(\boldsymbol{\varphi}, e, m, M)
$$

where $\boldsymbol{\varphi}=\left(\boldsymbol{\beta}, \varphi_{0}, \mathbf{u}, \mathbf{w}, W_{11}, W_{12}, W_{22}\right)$.

Now we introduce some notation which is used throughout the rest of the paper.

Definition 4.2. Let $\varrho>0$ be given by Condition (H.1). Denote by $\Sigma_{\varrho}$ the slab

$$
\Sigma_{\varrho}=\{(\alpha, k): \operatorname{Re} \alpha \in \mathbb{R},|\operatorname{Im} \alpha| \leq \varrho, \operatorname{Re} k \in[0,1],|\operatorname{Im} k| \leq \varrho\}
$$

Definition 4.3. Let $\varrho>0$ bi given by Condition $(\mathbf{H . 1})$. For every $\sigma \in[0,1]$ and any integer $d \geq 0$ denote by $\mathcal{A}_{\sigma, d}$ the Banach space of all functions

$$
\mathbf{u}: \Re_{\sigma \varrho} \rightarrow \mathbb{C}, \quad \Re_{\sigma \varrho}=\left\{\boldsymbol{\xi}: \operatorname{Re} \boldsymbol{\xi} \in \mathbb{T}^{n-1}, \quad|\operatorname{Im} \boldsymbol{\xi}| \leq \sigma \varrho\right\},
$$

with the finite norm

$$
\|u\|_{\sigma, d}=\max _{0 \leq|k| \leq d} \sup _{\boldsymbol{\xi} \in \Re_{\sigma \varrho}}\left|\partial^{k} u(\boldsymbol{\xi})\right| .
$$


Notice that system (4.9) consists of differential equations (4.9a)-(4.9d) and orthogonality conditions (4.9e)-(4.9g). It is convenient to incorporate the orthogonality conditions in the definition of the Banach spaces. Thus we arrive at the following

Definition 4.4. For every $\sigma \in[0,1]$ and any integer $d \geq 0$ denote by $\mathcal{E}_{\sigma, d}$ the subspace of the Banach space $\mathbb{C}^{n-1} \times \mathcal{A}_{\sigma, d} \times \mathcal{A}_{\sigma, d}^{n-1} \times \mathcal{A}_{\sigma, d}^{2} \times \mathcal{A}_{\sigma, d}^{3} \times \mathbb{C}^{3}$ which consists of all vectors

$$
\mathfrak{u}=(\boldsymbol{\varphi}, e, m, M) \text { with } \boldsymbol{\varphi}=\left(\boldsymbol{\beta}, \varphi_{0}, \mathbf{u}, \mathbf{w}, W_{11}, W_{12}, W_{21}\right),
$$

satisfying the orthogonality conditions (4.9g)-(4.9h).

Definition 4.5. For every $\sigma \in[0,1]$ and any integer $d \geq 0$ denote by $\mathcal{F}_{\sigma, d}$ the subspace of $\mathcal{A}_{\sigma, d} \times \mathcal{A}_{\sigma, d}^{n-1} \times \mathcal{A}_{\sigma, d}^{2} \times \mathcal{A}_{\sigma, d}^{4} \times \mathbb{C}$ which consists of all vectors

$$
\boldsymbol{F}=\left(F_{1}, F_{2}, F_{3}, F_{4}, f_{5}\right)
$$

such that $F_{4}$ is a symmetric $2 \times 2$-matrix valued function..

Introduce the nonlinear operator

$$
\begin{aligned}
& \mathbf{\Phi}(\mathfrak{u}, \mathfrak{f})= \\
& \left(\Phi_{1}(\boldsymbol{\varphi}, e, m, M), \quad \Phi_{2}(\boldsymbol{\varphi}, m, M), \Phi_{3}(\boldsymbol{\varphi}, m, M), \Phi_{4}(\boldsymbol{\varphi}, m, M ; k), \Phi_{5}(\mathbf{w} ; \alpha)\right),
\end{aligned}
$$

where $\Phi_{i}$ are given by (4.9). It is worthy noting that $\boldsymbol{\Phi}$ is a linear function of the scalars $e, m, M$, and $k, \alpha$. Hence the modified problem (4.9) can be written in the form of the operator equation

$$
\Phi(\mathfrak{f}, \mathfrak{u})=0 .
$$

Remark For $\varepsilon=0$ and $\mathfrak{f} \in \mathbb{C}^{2}$, equation (4.16) has the unique solution

$$
\mathfrak{v}(\mathfrak{f})=\left(\boldsymbol{\varphi}_{0}(\alpha),-k \alpha-k \alpha^{2},-k \alpha,-k\right),
$$

where

$$
\boldsymbol{\varphi}_{0}(\alpha)=\left(0,0, \alpha \mathbf{e}_{1}, 1,0,0\right)
$$

Notice that $\Phi$ is a nonlinear analytic differential operator. The general theory of the nonlinear differential operators in spaces of analytic functions $\mathcal{A}_{\sigma, d}$ is considered in the monograph [15] and [20]. We use the following proposition, see [15] Ch.6, which constitute the continuity and differentiability of the operator $\Phi$. 
Proposition 4.6. Let $\varrho>0$ be given by Condition (H.1). For every $\sigma \in$ $[0,1]$ and $d \geq 1$, there are $r>0$ and $c>0$ with the properties. If $\mathfrak{f} \in \Sigma_{\varrho}$ and $\mathfrak{u} \in \mathcal{E}_{\sigma, d}$ satisfy

$$
\left\|\boldsymbol{\varphi}-\boldsymbol{\varphi}_{0}(\alpha)\right\|_{\sigma, d} \leq r, \quad \boldsymbol{\varphi}_{0}=\left(0,0,0, \alpha \boldsymbol{e}_{1}, 1,0,0\right)
$$

then $\boldsymbol{\Phi}(\mathfrak{f}, \mathfrak{u}) \in \mathcal{F}_{\sigma, 0}$ and

$$
\|\mathbf{\Phi}(\mathfrak{f}, \mathfrak{u})\|_{\sigma, 0} \leq c+c(|e|+|m|+|M|) .
$$

If in addition

$$
\left\|\varphi+\delta \varphi-\varphi_{0}(\alpha)\right\|_{\sigma, d} \leq r
$$

then

$$
\begin{aligned}
& \Phi_{i}(\boldsymbol{\varphi}+\delta \boldsymbol{\varphi}, e, m, M)-\Phi_{i}(\boldsymbol{\varphi}, e, m, M)= \\
& D_{\varphi} \Phi_{i}(\boldsymbol{\varphi}, e, m, M)[\delta \boldsymbol{\varphi}]+Q_{\Phi_{i}}(\boldsymbol{\varphi}, \delta \boldsymbol{\varphi}, e, m, M) .
\end{aligned}
$$

The linear operator $D_{\varphi} \Phi_{i}$ and the remainder $Q_{\Phi_{i}}$ admit the estimates

$$
\begin{gathered}
\left\|D_{\varphi} \Phi_{i}(\boldsymbol{\varphi}, e, m, M)[\delta \boldsymbol{\varphi}]\right\|_{\sigma, d-1} \leq c(1+|e|+|m|+|M|)\|\delta \boldsymbol{\varphi}\|_{\sigma, d} \\
\left\|Q_{\Phi_{i}}(\boldsymbol{\varphi}, \delta \boldsymbol{\varphi}, e, m, M)\right\|_{\sigma, d-1} \leq c(1+|e|+|m|+|M|)\|\delta \boldsymbol{\varphi}\|_{\sigma, d}^{2}
\end{gathered}
$$

Third structural theorem. In order to apply the Nash-Moser implicit function theorem to operator equation (4.16), we have to prove that the linear operator $D_{\mathfrak{u}} \boldsymbol{\Phi}$ has an approximate inverse. In other words, we have to show that for every

$$
\mathbf{F}=\left(F_{1}, F_{2}, F_{3}, F_{4}, f_{5}\right) \in \mathcal{F}_{\sigma, d}
$$

the linear equation

$$
D_{\varphi} \boldsymbol{\Phi}(\boldsymbol{\varphi})[\delta \boldsymbol{\varphi}]+D_{e} \boldsymbol{\Phi}(\boldsymbol{\varphi})[\delta e]+D_{m} \boldsymbol{\Phi}(\boldsymbol{\varphi})[\delta m]+D_{M} \boldsymbol{\Phi}(\boldsymbol{\varphi})[\delta M]=\mathbf{F}
$$

has an analytic approximate solution $(\delta \boldsymbol{\varphi}, e, m, M)$. This problem looks like very difficult because of the complexity of the expression for $\delta \boldsymbol{\Phi}=$ $D_{\varphi} \boldsymbol{\Phi}(\boldsymbol{\varphi})[\delta \boldsymbol{\varphi}]$. The remarkable fact of KAM theory is that linear equation (4.21) can be reduced to the triangle system of first order differential equations with constant coefficients in the principle part. This very special change of variables is given by the second structural Theorem 3.2. In order to define, 
it choose an arbitrary vector $\delta \boldsymbol{\varphi}=\left(\delta \boldsymbol{\beta}, \delta \varphi_{0}, \delta \mathbf{u}, \delta \mathbf{w}, \delta W_{11}, \delta W_{12}, \delta W_{21}\right)$. Next notice that relations (3.20) in Theorem $3.2 \mathrm{read}$

$$
\begin{gathered}
\boldsymbol{\chi}=\mathbf{V}^{\top} \delta \mathbf{u} \\
\boldsymbol{\lambda}=\mathbf{W}^{-1} \delta \mathbf{w}-\chi_{i} \mathbf{W}^{-1} \frac{\partial}{\partial \xi_{i}} \mathbf{W} \\
\boldsymbol{\Gamma}=\mathbf{W}^{-1} \delta \mathbf{W}-\chi_{i} \mathbf{W}^{-1} \frac{\partial}{\partial \xi_{i}} \mathbf{W} \\
\boldsymbol{\mu}=\mathbf{V}^{-1}\left(\delta \mathbf{v}+\chi_{i} \frac{\partial}{\partial \xi_{i}} \mathbf{v}-\boldsymbol{\Lambda} \boldsymbol{\lambda}\right) \\
\nabla \psi_{0}=\boldsymbol{\mu}-\delta \boldsymbol{\beta}, \quad \delta \boldsymbol{\beta}=\frac{1}{(2 \pi)^{n-1}} \int_{\mathbb{T}^{n-1}} \boldsymbol{\mu} d \boldsymbol{\xi}
\end{gathered}
$$

where $\delta \mathbf{W}$ is given in terms of $\delta \boldsymbol{\varphi}$ by (3.11). These equalities completely define the vector

$$
\boldsymbol{\Upsilon}=\left(\delta \boldsymbol{\beta}, \psi_{0}, \boldsymbol{\chi}, \boldsymbol{\lambda}, \Gamma_{11}, \Gamma_{12}, \Gamma_{21}\right) .
$$

Recall that $\Gamma$ is $2 \times 2$ matrix such that $\Gamma_{22}=-\Gamma_{11}$. It is worth noting that equalities (4.22) establishes linear algebraic relations between $\delta \boldsymbol{\varphi}$ and $\Upsilon$ The following theorem shows that the change of variables (4.22) brings the linear operator $D_{\varphi} \Phi$ to the simple canonical form.

Theorem 4.7. Third Structure Theorem. Let $\boldsymbol{\varphi}, \alpha$ and $k$ meet all requirements of Proposition 4.6. Furthermore assume that

$$
\delta \Phi_{i}=D_{\varphi} \Phi_{i}(\boldsymbol{\varphi})[\delta \boldsymbol{\varphi}]
$$

Then for every $\delta \boldsymbol{\varphi} \in \mathbb{C}^{n-1} \times \mathcal{A}_{\sigma, d} \times \mathcal{A}_{\sigma, d}^{n-1} \times \mathcal{A}_{\sigma, d}^{2} \times \mathcal{A}_{\sigma, d}^{3}$,

$$
\begin{gathered}
\delta \Phi_{1}=\boldsymbol{\partial} \psi_{0}+\boldsymbol{\omega}^{\top} \cdot \delta \boldsymbol{\beta}+\Pi_{1}[\boldsymbol{\chi}, \boldsymbol{\mu}, \boldsymbol{\lambda}] \\
\delta \Phi_{2}=\mathbf{J} \boldsymbol{\partial} \boldsymbol{\lambda}+\boldsymbol{\Omega} \boldsymbol{\lambda}+\mathbf{T} \boldsymbol{\mu}+\Pi_{2}[\boldsymbol{\chi}, \boldsymbol{\lambda}, \boldsymbol{\Gamma}] \\
\delta \Phi_{3}=-\boldsymbol{\partial} \boldsymbol{\chi}+\mathbf{S} \boldsymbol{\mu}+\mathbf{T}^{\top} \boldsymbol{\lambda}+\Pi_{3}[\boldsymbol{\chi}] \\
\delta \Phi_{4}=\boldsymbol{\partial}(\mathbf{J} \boldsymbol{\Gamma})+\boldsymbol{\Omega} \boldsymbol{\Gamma}+(\boldsymbol{\Omega} \boldsymbol{\Gamma})^{\top}+\mathbf{U}_{i j} \frac{\partial \lambda_{i}}{\partial \xi_{j}}+\mu_{i} \mathbf{E}_{i}+\lambda_{i} \mathbf{K}_{i}+\Pi_{4}[\boldsymbol{\chi}, \boldsymbol{\Gamma}]
\end{gathered}
$$




$$
\delta \Phi_{5}=\frac{1}{(2 \pi)^{n-1}} \int_{\mathbb{T}^{n-1}}\left((\mathbf{W} \boldsymbol{\lambda})_{1}+\boldsymbol{\chi}_{i} \frac{\partial w_{1}}{\partial \xi_{i}}\right) d \boldsymbol{\xi} .
$$

Here $\delta \boldsymbol{\beta}, \psi_{0}, \boldsymbol{\chi}, \boldsymbol{\lambda}, \Gamma_{11}, \Gamma_{12}, \Gamma_{21}$ and $\delta \boldsymbol{\varphi}$ are interrelated by relations (4.22) . The linear differential operators $\Pi_{i}$ are defined by

$$
\begin{gathered}
\Pi_{1}[\boldsymbol{\chi}, \boldsymbol{\mu}, \boldsymbol{\mu}, \boldsymbol{\lambda}]=\frac{\partial \Phi_{1}}{\partial \xi_{i}} \chi_{i}+\Phi_{2}^{\top} \cdot \boldsymbol{\lambda}+\Phi_{3}^{\top} \cdot \boldsymbol{\mu}, \\
\Pi_{2}[\boldsymbol{\chi}, \boldsymbol{\lambda}, \boldsymbol{\Gamma}]=\chi_{i} \frac{\partial \Phi_{2}}{\partial \xi_{i}}+\boldsymbol{\Gamma}^{\top} \Phi_{2}+\Phi_{4} \boldsymbol{\lambda}+(\mathbf{J} \boldsymbol{\lambda})_{\xi}^{\prime} \Phi_{3}, \\
\Pi_{3}[\boldsymbol{\chi}]=\chi_{i} \frac{\partial \Phi_{3}}{\partial \xi_{i}}-\boldsymbol{\chi}_{\xi}^{\prime} \Phi_{3}, \\
\Pi_{4}[\boldsymbol{\chi}, \boldsymbol{\Gamma}]=\chi_{i} \frac{\partial \Phi_{4}}{\partial \xi_{i}}+\Phi_{3, i} \frac{\partial}{\partial \xi_{i}}(\mathbf{J} \boldsymbol{\Gamma})+\Phi_{4} \boldsymbol{\Gamma}+\left(\Phi_{4} \boldsymbol{\Gamma}\right)^{\top},
\end{gathered}
$$

where $\Phi_{i}=\Phi_{i}(\boldsymbol{\varphi}, e, m, M)$. The matrix $\mathbf{S}$ and $\mathbf{T}$ are given in terms of $\boldsymbol{\varphi}$ by the equalities

$$
\begin{array}{r}
\mathbf{S}=\mathbf{V}^{\top} \frac{\partial^{2} H}{\partial \mathbf{y}^{2}}(\boldsymbol{\xi}+\mathbf{u}, \mathbf{v}, \mathbf{w}) \mathbf{V} \\
\mathbf{T}=\mathbf{W}^{\top} \frac{\partial^{2} H}{\partial \mathbf{z} \partial \mathbf{y}}(\boldsymbol{\xi}+\mathbf{u}, \mathbf{v}, \mathbf{w}) \mathbf{V}+\boldsymbol{\Lambda}^{\top} \frac{\partial^{2} H}{\partial \mathbf{y}^{2}}(\boldsymbol{\xi}+\mathbf{u}, \mathbf{v}, \mathbf{w}) \mathbf{V}
\end{array}
$$

They admit the estimates

$$
\left\|\mathbf{S}-\mathbf{S}_{0}\right\|_{\sigma, d-1}+\left\|\mathbf{T}-\mathbf{T}_{0}\right\|_{\sigma, d-1} \leq c(\varepsilon+r),
$$

where $c$ depends only on $\sigma, d$ and $H$, the constant matrices $\mathbf{S}_{0}, \mathbf{T}_{0}$ are given by

$$
\mathbf{S}_{0}=\frac{\partial^{2} H_{0}}{\partial \mathbf{y}^{2}}(0,0), \quad \mathbf{T}_{0}=\frac{\partial^{2} H_{0}}{\partial \mathbf{z} \partial \mathbf{y}}(0,0)
$$

The symmetric matrix valued functions $\mathbf{U}_{i j}, \mathbf{E}_{i}, \mathbf{K}_{i}$ admit the estimates

$$
\left\|\mathbf{U}_{i j}\right\|_{\sigma, d-1}+\left\|\mathbf{E}_{i}\right\|_{\sigma, d-1}+\left\|\mathbf{K}_{i}\right\|_{\sigma, d-1} \leq c .
$$

Proof. The proof is in Appendix D.

Theorem 4.7 gives the representation of the derivatives $D_{\varphi} \boldsymbol{\Phi}=\delta \boldsymbol{\Phi}$. As corollary of this result we obtain the representation for the full differential $D_{\mathfrak{u}} \boldsymbol{\Phi}(\mathfrak{f}, \mathfrak{u})$ defined by

$$
D_{\mathfrak{u}} \boldsymbol{\Phi}(\mathfrak{f}, \mathfrak{u})[\delta \mathfrak{u}]=\lim _{\tau \rightarrow 0} \frac{1}{\tau}(\Phi(\mathfrak{f}, \mathfrak{u}+\tau \delta \mathfrak{u})-\Phi(\mathfrak{f}, \mathfrak{u})) .
$$

The result is given by the following 
Corollary 4.8. Under the assumptions of Theorem 4.7 the operators $D_{\mathfrak{u}} \Phi_{i}$ admit the representation

$$
\begin{gathered}
D_{\mathfrak{u}} \Phi_{1}(\mathfrak{f}, \mathfrak{u})[\delta \mathfrak{u}] \equiv \\
\boldsymbol{\partial} \psi_{0}+\boldsymbol{\omega}^{\top} \cdot \delta \boldsymbol{\beta}+\delta e+\delta m \cdot w_{1}+\frac{1}{2} \delta M w_{1}^{2}+\Pi_{1}[\boldsymbol{\chi}, \boldsymbol{\mu}, \boldsymbol{\lambda}], \\
D_{\mathfrak{u}} \Phi_{2}(\mathfrak{f}, \mathfrak{u})[\delta \mathfrak{u}] \equiv \\
\mathbf{J} \boldsymbol{\partial} \boldsymbol{\lambda}+\boldsymbol{\Omega} \boldsymbol{\lambda}+\mathbf{T} \boldsymbol{\mu}+\delta m \mathbf{W}^{\top} \mathbf{e}_{1}+\delta M w_{1} \mathbf{W}^{\top} \mathbf{e}_{1}+\Pi_{2}[\boldsymbol{\chi}, \boldsymbol{\lambda}, \boldsymbol{\Gamma}], \\
\boldsymbol{\mu}=\nabla \psi_{0}+\delta \boldsymbol{\beta} \\
D_{\mathfrak{u}} \Phi_{3}(\mathfrak{f}, \mathfrak{u})[\delta \mathfrak{u}] \equiv \\
-\boldsymbol{\partial} \boldsymbol{\chi}+\mathbf{S} \boldsymbol{\mu}+\mathbf{T}^{\top} \boldsymbol{\lambda}+\Pi_{3}[\boldsymbol{\chi}] \\
D_{\mathfrak{u}} \Phi_{4}(\mathfrak{f}, \mathfrak{u})[\delta \mathfrak{u}] \equiv \\
\boldsymbol{\partial}(\mathbf{J} \boldsymbol{\Gamma})+\boldsymbol{\Omega} \boldsymbol{\Gamma}+(\boldsymbol{\Omega} \boldsymbol{\Gamma})^{\top}+\mathbf{U}_{i j} \frac{\partial \lambda_{i}}{\partial \xi_{j}}+\mu_{i} \mathbf{E}_{i}+\lambda_{i} \mathbf{K}_{i}+ \\
\mathbf{W}^{\top} \delta \mathbf{M W}+\Pi_{4}[\boldsymbol{\chi}, \boldsymbol{\Gamma}] \\
D_{\mathfrak{u}} \Phi_{5}(\mathfrak{f}, \mathfrak{u})[\delta \mathfrak{u}] \equiv \\
\frac{1}{(2 \pi)^{n-1}} \int_{\mathbb{T}^{n-1}}\left((\mathbf{W} \boldsymbol{\lambda})_{1}+\boldsymbol{\chi}_{i} \frac{\partial w_{1}}{\partial \xi_{i}}\right) d \boldsymbol{\xi}
\end{gathered}
$$

Proof. Relations (4.9), (4.15) lead to the following expressions for $D_{e} \Phi, D_{m} \Phi$, and $D_{M} \Phi$

$$
\begin{gathered}
D_{e} \Phi_{1}[\delta e]=\delta e, \quad D_{e} \Phi_{i}[\delta e]=0 \quad \text { for } i=2,3,4,5 \\
D_{m} \Phi_{1}[\delta m]=\delta m w_{1}, \quad D_{m} \Phi_{2}[\delta m]=\delta m \mathbf{W}^{\top} \mathbf{e}_{1}, \\
D_{m} \Phi_{3}[\delta m]=0, \quad D_{m} \Phi_{4}[\mathbf{m}]=0, \quad D_{m} \Phi_{5}[\delta m]=0, \\
D_{M} \Phi_{1}[\delta M]=\frac{1}{2} w_{1}^{2} \delta M, \quad D_{M} \Phi_{2}[\delta M]=\mathbf{W}^{\top} \delta M \mathbf{w}, \\
D_{M} \Phi_{3}[\delta M]=0, \quad D_{M} \Phi_{4}[\delta M]=\mathbf{W}^{\top} \delta M \mathbf{W}, \quad D_{M} \Phi_{5}[\delta M]=0 .
\end{gathered}
$$

Substituting these expressions into the identity

$$
D_{\mathfrak{u}} \boldsymbol{\Phi}(\mathfrak{f}, \mathfrak{u})[\delta \mathfrak{u}]=\delta \boldsymbol{\Phi}+D_{e} \boldsymbol{\Phi}(\mathfrak{f}, \mathfrak{u}) \delta e+D_{m} \boldsymbol{\Phi}(\mathfrak{f}, \mathfrak{u}) \delta m+D_{M} \boldsymbol{\Phi}(\mathfrak{f}, \mathfrak{u}) \delta M
$$

and recalling expressions (4.23) for $\delta \boldsymbol{\Phi}_{i}$ in Theorem 4.7 we arrive at the desired identity (4.30). 


\section{Linear Problem}

In this section we prove the existence an approximate inverse of the Gateux derivative of the operator $\boldsymbol{\Phi}$. This result plays the crucial role in the proof of solvability of the modified problem (4.9). Choose an arbitrary $\sigma \in[1 / 2,1]$ and an arbitrary integer $d \geq 1$. Next, fix $\mathfrak{f}=(\alpha, k) \in \Sigma_{\varrho}$. In view of Corollary 4.8 the operator $\boldsymbol{\Phi}(\mathfrak{f}, \mathfrak{u})$ is differentiable with respect to $\mathfrak{u}$ for all $\mathfrak{u}=(\boldsymbol{\varphi}, e, m, M)$ satisfying the condition

$$
\left\|\varphi-\varphi_{0}(\alpha)\right\|_{\sigma, d} \leq r
$$

Notice that $\boldsymbol{\Phi}$ is a linear function of the parameters $e, m, M$. Consider the linear operator equation

$$
D_{\mathfrak{u}} \boldsymbol{\Phi}(\mathfrak{f}, \mathfrak{u})[\delta \mathfrak{u}]=\mathbf{F} \in \mathcal{F}_{\sigma, d}
$$

We are looking for a solution

$$
\delta \mathfrak{u}=(\delta \boldsymbol{\varphi}, \delta e, \delta m, \delta M)
$$

to this equation in the space $\mathcal{E}_{\sigma, d}$ given by Definition 4.4. Hence $\delta \boldsymbol{\varphi}$ have to satisfy the orthogonality conditions

$$
\int_{\mathbb{T}^{n-1}} \delta \varphi_{0} d \xi=0, \quad \int_{\mathbb{T}^{n-1}} \delta \mathbf{u} d \xi=0, \quad \int_{\mathbb{T}^{n-1}} \delta W_{12} d \xi=0
$$

which are similar condition (4.9h)-(4.9g) for $\varphi$. Relations (5.1)-(5.2) form the closed system of equations for the vector $\delta \mathfrak{u}$. However, the operator $D_{\mathfrak{u}} \boldsymbol{\Phi}$ has the complicated structure and its type is indefinite. In order to simplify the equation, we exploit Corollary 4.8 of Third Structural Theorem 4.7 and reduce equation (5.1) to the triangular canonical form with constant coefficients in the principal part. To this end we make the linear change of unknown functions and introduce the new unknown vector field $\Upsilon=\left(\delta \boldsymbol{\beta}, \psi_{0}, \boldsymbol{\lambda}, \boldsymbol{\chi}, \boldsymbol{\Gamma},\right)$ which is connected with

$$
\delta \boldsymbol{\varphi}=\left(\delta \boldsymbol{\beta}, \delta \varphi_{0}, \delta \mathbf{u}, \delta \mathbf{w}, \delta \mathbf{W}\right)
$$


by relations (4.22). Using identities (4.30) we rewrite equations (5.1)-(5.2) in the equivalent form

$$
\begin{aligned}
& \boldsymbol{\partial} \psi_{0}+\boldsymbol{\omega}^{\top} \cdot \delta \boldsymbol{\beta}+\Pi_{1}[\boldsymbol{\chi}, \boldsymbol{\mu}, \boldsymbol{\lambda}]+\delta e+\delta \mathbf{m} \cdot \mathbf{w}+\frac{1}{2} \mathbf{w}^{\top} \delta \mathbf{M w}=F_{1}, \\
& \mathbf{J} \boldsymbol{\partial} \boldsymbol{\lambda}+\mathbf{T} \boldsymbol{\mu}+\Pi_{2}[\boldsymbol{\chi}, \boldsymbol{\lambda}, \boldsymbol{\Gamma}]+\mathbf{W}^{\top} \delta \mathbf{m}+\mathbf{W}^{\top} \delta \mathbf{M w}=F_{2}, \\
& \boldsymbol{\mu}=\nabla \psi_{0}+\delta \boldsymbol{\beta} \\
& -\boldsymbol{\partial} \boldsymbol{\chi}+\mathbf{S} \boldsymbol{\mu}+\mathbf{T}^{\top} \boldsymbol{\lambda}+\Pi_{3}[\chi]=F_{3}, \\
& \boldsymbol{\partial}(\mathbf{J} \boldsymbol{\Gamma})+\boldsymbol{\Omega} \boldsymbol{\Gamma}+(\boldsymbol{\Omega} \boldsymbol{\Gamma})^{\top}+\mathbf{U}_{i j} \frac{\partial \lambda_{i}}{\partial \xi_{j}}+\mu_{i} \mathbf{E}_{i}+\lambda_{i} \mathbf{K}_{i} \\
& +\mathbf{W}^{\top} \delta \mathbf{M W}+\Pi_{4}[\chi, \Gamma]=F_{4} . \\
& (2 \pi)^{1-n} \int_{\mathbb{T}^{n-1}}\left((\mathbf{W} \boldsymbol{\lambda})_{1}+\chi_{i} \frac{\partial w_{1}}{\partial \xi_{i}}\right) d \boldsymbol{\xi}=f_{5}, \\
& (2 \pi)^{1-n} \int_{\mathbb{T}^{n-1}}\left[\psi_{0}-\delta \boldsymbol{\beta} \cdot \mathbf{u}+w_{2}(\mathbf{W} \boldsymbol{\lambda})_{1}\right] d \xi=0 \\
& (2 \pi)^{1-n} \int_{\mathbb{T}^{n-1}} \mathbf{V}^{-\top} \chi d \xi=0, \quad \int_{\mathbb{T}^{n-1}}(\mathbf{W} \boldsymbol{\Gamma})_{12} d \xi=0 .
\end{aligned}
$$

Recall that the matrices $\mathbf{S}$ and $\mathbf{T}$ are given by (4.25). This matrices along with the matrices $\mathbf{U}, \mathbf{E}_{i}$, and $\mathbf{K}_{i}$ admit estimates (4.27) and (4.28). The linear operators $\Pi$ are defined by formulae (4.24). They vanishes when $\Phi=0$.

\subsection{Approximate equations}

Relations (5.3) form the closed system of equations and the orthogonality conditions for the functions $\psi_{0}, \boldsymbol{\chi}, \boldsymbol{\lambda}, \boldsymbol{\Gamma}$, and the parameters $\boldsymbol{\beta}, \delta m, \delta M$, and $\delta e$. This system is not in a triangle form and it is inconvenient for the investigation. In order to cope with this difficulty we notice that, in accordance with the basic principles of the KAM theory, we are looking for an approximate solution to equations (5.3). This approximate solutions should satisfy equations (5.3) with the accuracy up to the discrepancy. Hence we can omit the operators $\Pi_{i}$ in $(\underline{5.3})$. Thus we arrive at the approximate 
equations

$$
\begin{aligned}
& \boldsymbol{\partial} \psi_{0}+\boldsymbol{\omega}^{\top} \cdot \delta \boldsymbol{\beta}+\delta e+\delta \mathbf{m} \cdot \mathbf{w}+\frac{1}{2} \mathbf{w}^{\top} \delta \mathbf{M w}=F_{1}, \\
& \mathbf{J} \boldsymbol{\partial} \boldsymbol{\lambda}+\boldsymbol{\Omega} \boldsymbol{\lambda}+\mathbf{T} \boldsymbol{\mu}+\mathbf{W}^{\top} \delta \mathbf{m}+\mathbf{W}^{\top} \delta \mathbf{M w}=F_{2}, \\
& \boldsymbol{\mu}=\nabla \psi_{0}+\delta \boldsymbol{\beta} \\
& -\boldsymbol{\partial} \chi+\mathbf{S} \boldsymbol{\mu}+\mathbf{T}^{\top} \boldsymbol{\lambda}=F_{3}, \\
& \boldsymbol{\partial}(\mathbf{J} \boldsymbol{\Gamma})+\boldsymbol{\Omega} \boldsymbol{\Gamma}+(\boldsymbol{\Omega} \boldsymbol{\Gamma})^{\top}+\mathbf{U}_{i j} \frac{\partial \lambda_{i}}{\partial \xi_{j}}+\mu_{i} \mathbf{E}_{i}+ \\
& \lambda_{i} \mathbf{K}_{i}+\mathbf{W}^{\top} \delta \mathbf{M W}=F_{4} . \\
& (2 \pi)^{1-n} \int_{\mathbb{T}^{n-1}}\left((\mathbf{W} \boldsymbol{\lambda})_{1}+\chi \cdot \nabla w_{1}\right) d \boldsymbol{\xi}=f_{5}, \\
& (2 \pi)^{1-n} \int_{\mathbb{T}^{n-1}}\left[\psi_{0}-\delta \boldsymbol{\beta} \cdot \beta \mathbf{u}+w_{2}(\mathbf{W} \boldsymbol{\lambda})_{1}\right] d \xi=0 \\
& (2 \pi)^{1-n} \int_{\mathbb{T}^{n-1}} \mathbf{V}^{-\top} \chi d \xi=0, \quad(2 \pi)^{1-n} \int_{\mathbb{T}^{n-1}}(\mathbf{W} \boldsymbol{\Gamma})_{12} d \xi=0,
\end{aligned}
$$

where $\delta \mathbf{m}=(\delta m, 0)^{\top}$ and $\delta \mathbf{M}=\operatorname{diag}(\delta M, 0)$.

The difficulty is that the "angle variable" $w_{1}$ is a growing function of $\alpha$. Hence the extra term $m w_{1}+2^{-1} M w_{1}^{2}$ in the modified Hamiltonian has a polynomial growth in $\alpha$. In other words, this means that the system of equations (5.4) contain secular terms. To cope with this difficulty we collect all secular terms together and introduce the new parameters

$$
\begin{array}{r}
q=\delta e+\delta \boldsymbol{\beta}^{\top} \cdot \boldsymbol{\omega}+\alpha \delta m+2^{-1} \alpha^{2} \delta M, \\
p=\delta m+\alpha \delta M, \quad \delta \mathbf{p}=(\delta p, 0)
\end{array}
$$

Recall that $\delta \boldsymbol{m}=(\delta m, 0), \delta \boldsymbol{M}=\operatorname{diag}(\delta M, 0)$. Thus we arrive at the following equations

$$
\begin{gathered}
\boldsymbol{\partial} \psi_{0}+q+p \cdot\left(w_{1}-\alpha\right)+\frac{1}{2} \delta M\left(w_{1}-\alpha\right)^{2}=F_{1}, \\
\mathbf{J} \boldsymbol{\partial} \boldsymbol{\lambda}+\boldsymbol{\Omega} \boldsymbol{\lambda}+\mathbf{T} \boldsymbol{\mu}+p \mathbf{W}^{\top} \mathbf{e}_{1}+\delta M\left(w_{1}-\alpha\right) \mathbf{W}^{\top} \mathbf{e}_{1}=F_{2}, \\
\boldsymbol{\mu}=\nabla \psi_{0}+\delta \boldsymbol{\beta} \\
-\boldsymbol{\partial} \boldsymbol{\chi}+\mathbf{S} \boldsymbol{\mu}+\mathbf{T}^{\top} \boldsymbol{\lambda}=F_{3}, \\
\boldsymbol{\partial}(\mathbf{J} \boldsymbol{\Gamma})+\boldsymbol{\Omega} \boldsymbol{\Gamma}+(\boldsymbol{\Omega} \boldsymbol{\Gamma})^{\top}+\mathbf{U}_{i j} \frac{\partial \lambda_{i}}{\partial \xi_{j}}+\mu_{i} \mathbf{E}_{i}+\lambda_{i} \mathbf{K}_{i}+ \\
+\mathbf{W}^{\top} \delta \mathbf{M W}=F_{4}, \quad \Gamma_{11}=-\Gamma_{22},
\end{gathered}
$$




$$
\begin{gathered}
(2 \pi)^{1-n} \int_{\mathbb{T}^{n-1}}\left((\mathbf{W} \boldsymbol{\lambda})_{1}+\boldsymbol{\chi} \cdot \nabla w_{1}\right) d \boldsymbol{\xi}=f_{5} \\
(2 \pi)^{1-n} \int_{\mathbb{T}^{n-1}}\left[\psi_{0}-\delta \boldsymbol{\beta} \cdot \mathbf{u}+w_{2}(\mathbf{W} \boldsymbol{\lambda})_{1}\right] d \xi=0 \\
(2 \pi)^{1-n} \int_{\mathbb{T}^{n-1}} \mathbf{V}^{-\top} \boldsymbol{\chi} d \xi=0, \quad(2 \pi)^{1-n} \int_{\mathbb{T}^{n-1}}(\mathbf{W} \boldsymbol{\Gamma})_{12} d \xi=0 .
\end{gathered}
$$

The following theorem constitutes the existence and uniqueness of solutions to problem (5.6)

Theorem 5.1. Let a fixed $\sigma \in[1 / 4,1], d \geq 2$, and the matrix $\mathbf{K}_{0}=\mathbf{S}_{0}-$ $\mathbf{t}_{0} \otimes \mathbf{t}_{0}$ given by (2.11), satisfies the condition det $\mathbf{K}_{0} \neq 0$. Then there are $\varepsilon_{0}>0$ and $r_{0}$ with the following properties. For every

$$
\begin{gathered}
(\alpha, k) \in \Sigma_{\varrho}, \quad\left\|\boldsymbol{\varphi}-\boldsymbol{\varphi}_{0}(\alpha)\right\|_{\sigma, d} \leq r_{0}, \quad \boldsymbol{\varphi}_{0}(\alpha)=\left(0,0,0, \alpha \mathbf{e}_{1}, 1,0,0\right), \quad|\varepsilon| \leq \varepsilon_{0}, \quad, \\
0 \leq \sigma_{0}<\sigma_{1} \leq \sigma, \quad \sigma_{1} \geq 1 / 4,
\end{gathered}
$$

and all $\mathbf{F}=\left(F_{1}, F_{2}, F_{3}, F_{4}, f_{5}\right) \in \mathcal{F}_{\sigma_{1}, 0}$, problem (5.6) has a unique solution

$$
\left(\psi_{0}, \boldsymbol{\lambda}, \boldsymbol{\chi}, \boldsymbol{\Gamma}, \delta \boldsymbol{\beta}, q, p, \delta M\right) \in \mathcal{A}_{\sigma_{0}, 0} \times \mathcal{A}_{\sigma_{0}, 0}^{2} \times \mathcal{A}_{\sigma, d}^{n-1} \times \mathcal{A}_{\sigma_{0}, 0}^{4} \times \mathbb{C}^{n-1} \times \mathbb{C}^{3} .
$$

This solution admits the estimate

$$
\left\|\left(\psi_{0}, \boldsymbol{\lambda}, \boldsymbol{\chi}, \boldsymbol{\Gamma}\right)\right\|_{\sigma_{0}, 0}+|(\delta \boldsymbol{\beta}, q, p, \delta M)| \leq c\left(\sigma_{1}-\sigma_{0}\right)^{-8 n-12}\|\mathbf{F}\|_{\sigma_{1}, 0}
$$

where the constant $c$ is independent of $\varepsilon_{0}, r_{0}$, and $\sigma_{i}$.

Proof. The proof is in Appendix E.1.

Notice that problems (5.4) and (5.6) are equivalent. The vector $\boldsymbol{\Upsilon}=$ $\left(\delta \boldsymbol{\beta}, \psi_{0}, \boldsymbol{\lambda}, \boldsymbol{\chi}, \boldsymbol{\Gamma}\right)$ and the parameters $\delta e, \delta m$, and $\delta M$ satisfy equations (5.4) if and only if $\Upsilon, \delta M$ and the parameters $\delta q, \delta p$, given by (5.5), satisfy equations (5.6). Thus we obtain the following

Corollary 5.2. Under the assumptions of Theorem 5.1, problem (5.4) has a unique solution

$$
\left(\psi_{0}, \boldsymbol{\lambda}, \boldsymbol{\chi}, \boldsymbol{\Gamma}, \delta \boldsymbol{\beta}, \delta e, \delta m, \delta M\right) \in \mathcal{A}_{\sigma_{0}, 0} \times \mathcal{A}_{\sigma_{0}, 0}^{2} \times \mathcal{A}_{\sigma, d}^{n-1} \times \mathcal{A}_{\sigma_{0}, 0}^{4} \times \mathbb{C}^{n-1} \times \mathbb{C}^{3}
$$

This solution admits the estimate

$$
\begin{gathered}
\left\|\left(\psi_{0}, \boldsymbol{\lambda}, \boldsymbol{\chi}, \boldsymbol{\Gamma}\right)\right\|_{\sigma_{0}, 0}+|(\delta \boldsymbol{\beta}, \delta M)| \leq c\left(\sigma_{1}-\sigma_{0}\right)^{-8 n-12}\|\mathbf{F}\|_{\sigma_{1}, 0} \\
|(\delta e, \delta m)| \leq\left(1+\alpha^{2}\right) c\left(\sigma_{1}-\sigma_{0}\right)^{-8 n-12}\|\mathbf{F}\|_{\sigma_{1}, 0}
\end{gathered}
$$




\subsection{Differential of $\Phi$. Approximate inverse}

In this section we construct an approximate inverse to the operator $D \boldsymbol{\Phi}$. Our considerations are based on the following construction. For given $\boldsymbol{\varphi}$ denote by $\Xi(\varphi)$ the linear operator defined by the equalities

$$
\begin{aligned}
& \Xi: \Upsilon=\left(\delta \boldsymbol{\beta}, \boldsymbol{\psi}_{0}, \boldsymbol{\lambda}, \boldsymbol{\chi}, \boldsymbol{\Gamma}\right) \rightarrow \delta \boldsymbol{\varphi}=\left(\delta \boldsymbol{\beta}, \delta \varphi_{0}, \delta \mathbf{u}, \delta \mathbf{w}, \delta W_{11}, \delta W_{12}, \delta W_{21}\right) \\
& \delta \mathbf{u}=\chi_{i} \frac{\partial}{\partial \xi_{i}}(\boldsymbol{\xi}+\mathbf{u}) \\
& \delta \mathbf{w}=\mathbf{W} \boldsymbol{\lambda}+\chi_{i} \frac{\partial}{\partial \xi_{i}} \mathbf{w} \\
& \delta \mathbf{W}=\mathbf{W} \boldsymbol{\Gamma}+\chi_{i} \frac{\partial}{\partial \xi_{i}} \mathbf{W}, \\
& \delta \varphi_{0}=\psi_{0}+w_{2} \delta w_{1}+\left(\frac{\partial \varphi_{0}}{\partial \xi_{i}}-w_{2} \frac{\partial w_{1}}{\partial \xi_{i}}\right) \chi_{i}-\mathbf{u} \cdot \delta \boldsymbol{\beta} .
\end{aligned}
$$

In view of the Second structural Theorem 3.2 the operator $\Xi$ has the inverse $\Xi^{-1}$ defined by

$$
\begin{gathered}
\Xi^{-1} \delta \boldsymbol{\varphi}=\left(\delta \boldsymbol{\beta}, \delta \varphi_{0}, \delta \mathbf{u}, \delta \mathbf{w}, \delta W_{11}, \delta W_{12}, \delta W_{21}\right) \boldsymbol{\Upsilon}=\left(\delta \boldsymbol{\beta}, \boldsymbol{\psi}_{0}, \boldsymbol{\lambda}, \boldsymbol{\chi}, \boldsymbol{\Gamma}\right) \\
\boldsymbol{\chi}=\mathbf{V}^{\top} \delta \mathbf{u} \\
\boldsymbol{\lambda}=\mathbf{W}^{-1} \delta \mathbf{w}-\chi_{i} \mathbf{W}^{-1} \frac{\partial}{\partial \xi_{i}} \mathbf{w} \\
\boldsymbol{\Gamma}=\mathbf{W}^{-1} \delta \mathbf{W}-\chi_{i} \mathbf{W}^{-1} \frac{\partial}{\partial \xi_{i}} \mathbf{W} \\
\boldsymbol{\mu}=\mathbf{V}^{-1}\left(\delta \mathbf{v}+\chi_{i} \frac{\partial}{\partial \xi_{i}} \mathbf{v}-\boldsymbol{\Lambda} \boldsymbol{\lambda}\right) \\
\psi_{0}=\delta \varphi_{0}-\chi_{i} \frac{\partial \varphi_{0}}{\partial \xi_{i}}+w_{2} \chi_{i} \frac{\partial w_{1}}{\partial \xi_{i}}+\delta \boldsymbol{\beta}^{\top} \cdot \mathbf{u} .
\end{gathered}
$$

Recall the notation

$$
\mathfrak{f}=(\alpha, k), \quad \mathfrak{u}=(\boldsymbol{\varphi}, e, m, M)
$$

Introduce also the notation

$$
\delta \mathfrak{u}=(\delta \varphi, \delta e, \delta m, \delta M) .
$$


Definition 5.3. Let $\mathcal{R}(\mathfrak{f}, \mathfrak{u})$ be a linear operator defined by the equality

$$
\mathcal{R}(\mathfrak{f}, \mathfrak{u})[\mathbf{F}]=(\boldsymbol{\Xi}(\boldsymbol{\varphi})[\mathbf{\Upsilon}], \delta e, \delta m, \delta M)
$$

where $\Upsilon$, $\delta e, \delta m, \delta M$ is a solution to the approximate problem (5.4). Notice that the coefficients of equations (5.4) are completely defined by $\mathfrak{f}$ and $\mathfrak{u}$.

The following proposition constitutes the basic properties of the operator $\mathcal{R}$. In particular, it follows that $\mathcal{R}$ is an approximate inverse to the operator $D \Phi$.

Proposition 5.4. Let $\sigma \in(0,1]$. Then there are $\varepsilon_{0}>0$ and $r_{0}>0$ with the following properties. For all $\mathfrak{f}, \mathfrak{u}$, and $\varepsilon$, and $\sigma_{i}$ satisfying the conditions

$$
\begin{aligned}
\mathfrak{f} \in \Sigma_{\varrho},|\varepsilon| & \leq \varepsilon_{0},\left\|\boldsymbol{\varphi}-\boldsymbol{\varphi}_{0}(\alpha)\right\|_{\sigma, d} \leq r_{0}, \\
0 & \leq \sigma_{0}<\sigma_{1} \leq \sigma
\end{aligned}
$$

the operator $\mathcal{R}(\mathfrak{f}, \mathfrak{u}): \mathcal{F}_{\sigma_{1}, 0} \rightarrow \mathcal{E}_{\sigma_{0}, d}$ is bounded and admits the estimate

$$
\|\mathcal{R}(\mathfrak{f}, \mathfrak{u})[\delta \mathfrak{u}]\|_{\sigma_{0}, d} \leq c\left(1+\alpha^{2}\right)\left(\sigma_{1}-\sigma_{0}\right)^{-8 n-12-d}\|\delta \mathfrak{u}\|_{\mathcal{E}_{\sigma_{1}, d}}
$$

Moreover, it satisfies the inequalities

$$
\begin{gathered}
\|(\mathcal{R}(\mathfrak{f}, \mathfrak{u}) D \Phi(\mathfrak{f}, \mathfrak{u})-\mathrm{Id})[\delta \mathfrak{u}]\|_{\sigma_{0}, d} \leq \\
c\left(\sigma_{1}-\sigma_{0}\right)^{-8 n-13-2 d}\|\mathbf{\Phi}(\mathfrak{f}, \mathfrak{u})\|_{\sigma, 0}\|\delta \mathfrak{u}\|_{\mathcal{E}_{\sigma_{1}, d}} \\
\|(D \Phi(\mathfrak{f}, \mathfrak{u}) \mathcal{R}(\mathfrak{f}, \mathfrak{u})-\mathrm{Id})[\mathbf{F}] \\
\begin{array}{c}
\| \\
\left.\quad c\left(\sigma_{\sigma_{0}, 0}-\sigma_{0}\right)^{-8 n-14} \| \mathbf{\Phi}(\mathfrak{f}, \mathfrak{u})\right)\left\|_{\sigma_{1}, 0}\right\| \mathbf{F} \|_{\sigma_{1}, 0}
\end{array}
\end{gathered}
$$

i.e., $\mathcal{R}$ is an approximate inverse to $D \boldsymbol{\Phi}$.

Proof. The proof is based on the following auxiliary lemma .

Lemma 5.5. Let a fixed $\sigma \in(0,1)$ and $d \geq 2$ and $r_{0}<1 / 2$. Then for every

$$
\mathfrak{f} \in \Sigma_{\varrho}, \quad\left\|\boldsymbol{\varphi}-\boldsymbol{\varphi}_{0}(\alpha)\right\|_{\sigma, d} \leq r_{0}, \quad \boldsymbol{\varphi}_{0}(\alpha)=(0,0,0, \boldsymbol{\alpha}, 1,0,0),
$$

and every

$$
0 \leq \sigma_{0}<\sigma_{1} \leq \sigma
$$


the operator

$\Xi: \mathbb{C} \times \mathcal{A}_{\sigma_{1}, 0} \times \mathcal{A}_{\sigma_{1}, 0}^{2} \times \mathcal{A}_{\sigma_{1}, 0}^{n-1} \times \mathcal{A}_{\sigma_{1}, 0}^{4} \rightarrow \mathbb{C} \times \mathcal{A}_{\sigma_{0}, d} \times \mathcal{A}_{\sigma_{0}, d}^{2} \times \mathcal{A}_{\sigma_{1}, d}^{n-1} \times \mathcal{A}_{\sigma_{0}, d}^{3}$

is well defined and admits the estimate

$$
\|\boldsymbol{\Xi}(\boldsymbol{\varphi})[\mathbf{\Upsilon}]\|_{\sigma_{0}, d} \leq c\left(\sigma_{1}-\sigma_{0}\right)^{-d}\|\boldsymbol{\Upsilon}\|_{\sigma_{1}, 0}
$$

The inverse operator

$\boldsymbol{\Xi}^{-1}: \mathbb{C} \times \mathcal{A}_{\sigma_{1}, 0} \times \mathcal{A}_{\sigma_{1}, 0}^{2} \times \mathcal{A}_{\sigma_{1}, 0}^{n-1} \times \mathcal{A}_{\sigma_{1}, 0}^{4} \rightarrow \mathbb{C} \times \mathcal{A}_{\sigma_{0}, d} \times \mathcal{A}_{\sigma_{0}, d}^{2} \times \mathcal{A}_{\sigma_{1}, d}^{n-1} \times \mathcal{A}_{\sigma_{0}, d}^{3}$

is well defined and admits the estimate

$$
\left\|\boldsymbol{\Xi}^{-1}(\boldsymbol{\varphi})[\delta \boldsymbol{\varphi}]\right\|_{\sigma_{0}, d} \leq c\left(\sigma_{1}-\sigma_{0}\right)^{-d}\|\delta \boldsymbol{\varphi}\|_{\sigma_{1}, 0}
$$

Proof. In order to avoid repetitions we prove estimate (5.16). The proof of estimate (5.17) is similar. Notice that for $d \geq 1$ and $r_{0} \leq 1 / 2$, the equality $W_{22}=W_{11}^{-1}\left(1-W_{1,2} W_{21}\right)$ implies

$$
\left\|w_{2}\right\|_{\sigma, 0}+\left\|\nabla w_{1}\right\|_{\sigma, 0}+\|\nabla \boldsymbol{W}\|_{\sigma, 0}+\|\boldsymbol{W}-\mathbf{I}\|_{\sigma, 0} \leq c\|\boldsymbol{\varphi}-\boldsymbol{\varphi}(\alpha)\|_{\sigma, d}
$$

Obviously we have

$$
\left\|w_{2}\right\|_{\sigma, 0}+\left\|\nabla w_{1}\right\|_{\sigma, 0}+\|\nabla(\boldsymbol{\xi}+\boldsymbol{u})\|+\|\boldsymbol{W}\|_{\sigma, 0} \leq c\|\boldsymbol{\varphi}-\boldsymbol{\varphi}(\alpha)\|_{\sigma, d} \leq c
$$

Since $\mathcal{A}_{\sigma_{1}, 0}$ is a Banach algebra, it follows from this and (5.10) that

$$
\left.\|\boldsymbol{\Xi}(\boldsymbol{\varphi})[\Upsilon]\|_{\sigma_{1}, 0} \leq c \| \Upsilon\right] \|_{\sigma_{1}, 0}
$$

It remains to note that

$$
\|\boldsymbol{\Xi}(\boldsymbol{\varphi})[\boldsymbol{\Upsilon}]\|_{\sigma_{0}, d} \leq c\left(\sigma_{1}-\sigma_{0}\right)^{-d}\|\boldsymbol{\Xi}(\boldsymbol{\varphi})[\boldsymbol{\Upsilon}]\|_{\sigma_{1}, 0}
$$

Let us turn to the proof of the proposition. The proof falls into three steps.

Step 1. We begin with proving of estimate (5.13). It follows from Corollary 5.2 that, under the assumptions of Proposition 5.4, for a suitable choice 
of $\varepsilon_{0}$ and $r_{0}$, problem (5.4) has the unique solution $\Upsilon=\left(\delta \boldsymbol{\beta}, \psi_{0}, \boldsymbol{\lambda}, \boldsymbol{\chi}, \boldsymbol{\Gamma}\right)$, and $\delta e, \delta m, \delta M$ satisfying the inequality

$$
\begin{aligned}
\|\Upsilon\|_{\left(\sigma_{0}+\sigma_{1}\right) / 2,0}+|\delta e|+|\delta m|+\mid & \delta M \mid \\
& \leq c\left(1+\alpha^{2}\right)\left(\sigma_{1}-\sigma_{0}\right)^{-8 n-12}\|\mathbf{F}\|_{\sigma_{1}, 0}
\end{aligned}
$$

Using this estimate and applying Lemma 5.5 with $\sigma_{1}$ replaced by $\left(\sigma_{0}+\sigma_{1}\right) / 2$ we obtain

$$
\begin{aligned}
\|\boldsymbol{\Xi}(\boldsymbol{\varphi})[\boldsymbol{\Upsilon}]\|_{\sigma_{0}, d} & \left.\leq c\left(\sigma_{1}-\sigma_{0}\right)^{-d} \| \boldsymbol{\Upsilon}\right] \|_{\left(\sigma_{0}+\sigma_{1}\right) / 2,0} \\
& \leq c\left(\sigma_{1}-\sigma_{0}\right)^{-8 n-12-d}\|\mathbf{F}\|_{\sigma_{1}, 0}
\end{aligned}
$$

Combining (5.18) and (5.19) and recalling Definition 5.3 we arrive at (5.13).

Step 2. Now our task is to prove estimate (??). Assume that $\varepsilon, r_{0}$ meet all requirements of Corollary 5.2. Choose an arbitrary

$$
\delta \boldsymbol{\varphi}=\left(\delta \boldsymbol{\beta}, \delta \varphi_{0}, \delta \mathbf{u}, \delta W_{11}, \delta W_{12}, \delta W_{21}\right) \quad \text { and }(\delta e, \delta m, \delta M)
$$

such that

$$
\delta \mathfrak{u} \equiv(\delta \varphi, \delta e, \delta m, \delta M) \in \mathcal{E}_{\sigma_{1}, d}
$$

Set

$$
\Upsilon=\Xi^{-1}(\varphi)[\delta \varphi]
$$

By construction, the vector

$$
\Upsilon=\left(\delta \boldsymbol{\beta}, \psi_{0}, \boldsymbol{\lambda}, \boldsymbol{\chi}, \boldsymbol{\Gamma}\right)
$$

is connected with $\delta \boldsymbol{\varphi}$ by relations (4.22). Hence $\Upsilon$ and the parameters $(\delta e, \delta m, \delta M)$ meet all requirements of Corollary 4.8. It follows that they satisfy identities (4.30). Notice that relations (4.30) can be regarded as system of equations (5.4a) $-(5.4 \mathrm{e})$ with the right hand sides

$$
\begin{array}{r}
F_{1}=-\Pi_{1}[\boldsymbol{\chi}, \boldsymbol{\lambda}, \boldsymbol{\mu}]+D_{\mathfrak{u}} \Phi_{1}(\mathfrak{f}, \mathfrak{u})[\delta \mathfrak{u}], \\
F_{2}=-\Pi_{2}[\boldsymbol{\chi}, \boldsymbol{\lambda}, \boldsymbol{\Gamma}]+D_{\mathfrak{u}} \Phi_{2}(\mathfrak{f}, \mathfrak{u})[\delta \mathfrak{u}], \\
F_{3}=-\Pi_{3}[\boldsymbol{\chi}]+D_{\mathfrak{u}} \Phi_{3}(\mathfrak{f}, \mathfrak{u})[\delta \mathfrak{u}], \\
F_{4}=-\Pi_{2}[\boldsymbol{\chi}, \boldsymbol{\Gamma}]+D_{\mathfrak{u}} \Phi_{4}(\mathfrak{f}, \mathfrak{u})[\delta \mathfrak{u}], \\
f_{5}=D_{\mathfrak{u}} \Phi_{5}(\mathfrak{f}, \mathfrak{u})[\delta \mathfrak{u}]
\end{array}
$$


Moreover, since $\delta \mathfrak{u} \in \mathcal{E}_{\sigma_{1}, d}$, its component $\delta \boldsymbol{\varphi}$ satisfies the integral relations (5.2) which, in view of (5.10b)-(5.10e), are equivalent to the integral conditions (5.4f) $-(5.4 \mathrm{~g})$. Therefore, the vector $\Upsilon$ and the parameters $(\delta e, \delta m, \delta M)$ satisfy equations (5.4) with the right hand sides given by (5.21).

On the other hand, Definition 5.3 implies the identity

$$
\mathcal{R}(\mathfrak{f}, \mathfrak{u})\left[D_{\mathfrak{u}} \boldsymbol{\Phi}(\mathfrak{f}, \mathfrak{u})[\delta \mathfrak{u}]\right]=\left(\boldsymbol{\Xi}(\boldsymbol{\varphi})\left[\mathbf{\Upsilon}^{\prime}\right], \delta e^{\prime}, \delta m^{\prime}, \delta M^{\prime}\right)
$$

where the vector $\Upsilon^{\prime}$ and the parameters $\left(\delta e^{\prime}, \delta m^{\prime}, \delta M^{\prime}\right)$ satisfy equations (5.4) with the right hand sides

$$
F_{i}^{\prime}=D \Phi_{i}(\mathfrak{f}, \mathfrak{u})[\delta \mathfrak{u}], i=1, \ldots, 4, f_{5}^{\prime}=D \Phi_{5}(\mathfrak{f}, \mathfrak{u})[\delta \mathfrak{u}]
$$

It follows from (5.20) that

$$
\mathcal{R}(\mathfrak{f}, \mathfrak{u})\left[D_{\mathfrak{u}} \boldsymbol{\Phi}(\mathfrak{f}, \mathfrak{u})[\delta \mathfrak{u}]\right]-\delta \mathfrak{u}=(\boldsymbol{\Xi}(\boldsymbol{\varphi})[\tilde{\mathbf{\Upsilon}}], \tilde{\delta e}, \tilde{\delta m}, \delta \tilde{M})
$$

Where

$$
\tilde{\Upsilon}=\Upsilon^{\prime}-\Upsilon \mathbf{\Upsilon}, \quad \tilde{\delta}=\delta e^{\prime}-\delta e, \quad \tilde{\delta m}=\delta m^{\prime}-\delta m, \quad \delta \tilde{M}=\delta M^{\prime}-\delta M .
$$

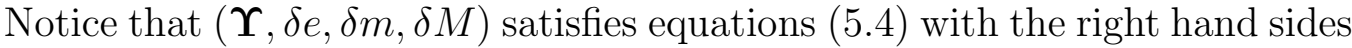
(5.21). On the other hand, $\left(\mathbf{\Upsilon}^{\prime}, \delta e^{\prime}, \delta m^{\prime}, \delta M^{\prime}\right)$ satisfies equations (5.4) with the right hand sides (5.23). It follows from this that $(\tilde{\Upsilon}, \tilde{\delta e}, \tilde{\delta m}, \delta \tilde{M})$ satisfies equations (5.4) with the right hand sides

$$
\tilde{F}_{i}=\Pi_{i}[\mathbf{\Upsilon}], i=1, \ldots, 4, \tilde{f}_{5}=0 .
$$

Here the operators $\Pi_{i}$ are given by formulae (4.24):

$$
\begin{gathered}
\Pi_{1}[\boldsymbol{\Upsilon}]=\frac{\partial \Phi_{1}}{\partial \xi_{i}} \chi_{i}+\Phi_{2}^{\top} \cdot \boldsymbol{\lambda}+\Phi_{3}^{\top} \cdot \boldsymbol{\mu} \\
\Pi_{2}[\boldsymbol{\chi}, \boldsymbol{\lambda}, \boldsymbol{\Gamma}]=\chi_{i} \frac{\partial \Phi_{2}}{\partial \xi_{i}}+\boldsymbol{\Gamma}^{\top} \Phi_{2}+\Phi_{4} \boldsymbol{\lambda}+(\mathbf{J} \boldsymbol{\lambda})_{\xi}^{\prime} \Phi_{3}, \\
\Pi_{3}[\boldsymbol{\chi}]=\chi_{i} \frac{\partial \Phi_{3}}{\partial \xi_{i}}-\chi_{\xi}^{\prime} \Phi_{3}, \\
\Pi_{4}[\boldsymbol{\chi}, \boldsymbol{\Gamma}]=\chi_{i} \frac{\partial \Phi_{4}}{\partial \xi_{i}}+\Phi_{3, i} \frac{\partial}{\partial \xi_{i}}(\mathbf{J} \boldsymbol{\Gamma})+\Phi_{4} \boldsymbol{\Gamma}+\left(\Phi_{4} \boldsymbol{\Gamma}\right)^{\top}
\end{gathered}
$$


where $\Phi_{i}=\Phi_{i}(\mathfrak{f}, \mathfrak{u})$ and $\boldsymbol{\mu}=\delta \boldsymbol{\beta}+\nabla \psi_{0}$. We thus get

$$
\begin{aligned}
& \boldsymbol{\partial} \tilde{\psi}_{0}+\boldsymbol{\omega}^{\top} \cdot \delta \tilde{\boldsymbol{\beta}}+\tilde{\delta e}+\tilde{\delta \mathbf{m}} \cdot \mathbf{w}+\frac{1}{2} \mathbf{w}^{\top} \delta \tilde{\mathbf{M}} \mathbf{w}=\tilde{F}_{1}, \\
& \mathbf{J} \boldsymbol{\partial} \tilde{\boldsymbol{\lambda}}+\boldsymbol{\Omega} \tilde{\boldsymbol{\lambda}}+\mathbf{T} \tilde{\boldsymbol{\mu}}+\delta \tilde{m} \mathbf{W}^{\top} \mathbf{e}_{1}+\mathbf{W}^{\top} \delta \tilde{\mathbf{M}} \mathbf{w}=\tilde{F}_{2}, \\
& \tilde{\boldsymbol{\mu}}=\nabla \tilde{\psi}_{0}+\delta \tilde{\boldsymbol{\beta}} \\
& -\boldsymbol{\partial} \tilde{\chi}+\mathbf{S} \tilde{\boldsymbol{\mu}}+\mathbf{T}^{\top} \tilde{\boldsymbol{\lambda}}=\tilde{F}_{3}, \\
& \boldsymbol{\partial}(\mathbf{J} \tilde{\boldsymbol{\Gamma}})+\boldsymbol{\Omega} \tilde{\boldsymbol{\Gamma}}+(\boldsymbol{\Omega} \tilde{\boldsymbol{\Gamma}})^{\top}+\mathbf{U}_{i j} \frac{\partial \lambda_{i}}{\partial \xi_{j}^{*}}+\tilde{\mu}_{i} \mathbf{E}_{i}+ \\
& \tilde{\lambda}_{i} \mathbf{K}_{i}+\mathbf{W}^{\top} \delta \tilde{\mathbf{M}} \mathbf{W}=\tilde{F}_{4} \text {. } \\
& \int_{\mathbb{T}^{n-1}}\left((\mathbf{W} \tilde{\boldsymbol{\lambda}})_{1}+\tilde{\boldsymbol{\chi}} \cdot \nabla w_{1}\right) d \boldsymbol{\xi}=0, \\
& \int_{\mathbb{T}^{n-1}}\left[\tilde{\psi}_{0}-\delta \tilde{\boldsymbol{\beta}} \cdot \mathbf{u}+w_{2}(\mathbf{W} \tilde{\boldsymbol{\lambda}})_{1}\right] d \xi=0 \\
& \int_{\mathbb{T}^{n-1}} \mathbf{V}^{-\top} \tilde{\boldsymbol{\chi}} d \xi=0, \quad \int_{\mathbb{T}^{n-1}}(\mathbf{W} \tilde{\boldsymbol{\Gamma}})_{12} d \xi=0,
\end{aligned}
$$

where $\delta \tilde{\mathbf{M}}=\operatorname{diag}(\delta \tilde{M}, 0)$. Let us estimate $\tilde{\mathbf{F}}$. Equality $\boldsymbol{\Upsilon}=\boldsymbol{\Xi}^{-1} \boldsymbol{\delta} \varphi$ and estimate (5.17) imply

$$
\|\Upsilon\|_{\left(\sigma_{1}+\sigma_{0}\right) / 2, d} \leq c\left(\sigma_{1}-\sigma_{0}\right)^{-d}\|\delta \boldsymbol{\varphi}\|_{\sigma_{1}, 0}
$$

It follows from this that

$$
\|\Upsilon\|_{\left(2 \sigma_{1}+\sigma_{0}\right) / 3,0}+\left\|\partial_{\xi} \Upsilon\right\|_{\left(2 \sigma_{1}+\sigma_{0}\right) / 3,0} \leq c\left(\sigma_{1}-\sigma_{0}\right)^{-d}\|\delta \boldsymbol{\varphi}\|_{\sigma_{1}, 0}
$$

Next we have

$$
\|\boldsymbol{\Phi}\|_{\left(2 \sigma_{1}+\sigma_{0}\right) / 3,0}+\left\|\partial_{\xi} \boldsymbol{\Phi}\right\|_{\left(2 \sigma_{1}+\sigma_{0}\right) / 3,0} \leq c\left(\sigma_{1}-\sigma_{0}\right)^{-1}\|\boldsymbol{\Phi}\|_{\sigma_{1}, 0}
$$

Combining estimates (5.28) and (15.29) and recalling formulae (5.26) we arrive at

$$
\|\Pi[\Upsilon]\|_{\left(2 \sigma_{1}+\sigma_{0}\right) / 3,0} \leq c\left(\sigma_{1}-\sigma_{0}\right)^{-d-1}\|\boldsymbol{\Phi}\|_{\sigma_{1}, 0}\|\delta \boldsymbol{\varphi}\|_{\sigma_{1}, 0}
$$

Since $\delta \boldsymbol{\varphi}$ is the component of the vector $\mathfrak{u}$, it follows from this and (5.25) that

$$
\|\tilde{\mathbf{F}}\|_{\left(2 \sigma_{1}+\sigma_{0}\right) / 3,0} \leq c\left(\sigma_{1}-\sigma_{0}\right)^{-d-1}\|\boldsymbol{\Phi}\|_{\sigma_{1}, 0}\|\delta \mathfrak{u}\|_{\sigma_{1}, 0}
$$


Applying Corollary 5.2 to problem (5.27) we conclude that it has the unique solution. Moreover, estimates (5.8) and (5.9) in this corollary imply the estimate

$$
c\left(1+\left|\alpha^{2}\right|\right)\left(\sigma_{1}-\sigma_{0}\right)^{-8 n-12}\|\tilde{\mathbf{F}}\|_{\left(2 \sigma_{1}+\sigma_{0}\right) / 3,0}
$$

This result along with inequality (5.31) leads to the estimate

$$
\begin{array}{r}
\|\tilde{\boldsymbol{\Upsilon}}\|_{\left(\sigma_{1}+\sigma_{0}\right) / 2,0}+|\delta \tilde{e}|+|\delta \tilde{m}|+|\delta \tilde{M}| \leq \\
c\left(1+\left|\alpha^{2}\right|\right)\left(\sigma_{1}-\sigma_{0}\right)^{-8 n-13-d}\|\boldsymbol{\Phi}\|_{\sigma_{1}, 0}\|\delta \mathfrak{u}\|_{\sigma_{1}, 0} .
\end{array}
$$

From this, relation (5.24) and estimate (5.16) for the norm of the operator $\boldsymbol{\Xi}$ we obtain

$$
\begin{gathered}
\left\|\mathcal{R}(\mathfrak{f}, \mathfrak{u})\left[D_{\mathfrak{u}} \boldsymbol{\Phi}(\mathfrak{f}, \mathfrak{u})[\delta \mathfrak{u}]\right]-\delta \mathfrak{u}\right\|_{\sigma_{0}, d}= \\
\|\boldsymbol{\Xi}(\boldsymbol{\varphi})[\tilde{\boldsymbol{\Upsilon}}]\|_{\sigma_{0}, d}+|\delta \tilde{e}|+|\delta \tilde{m}|+|\delta \tilde{M}| \leq \\
\left(\sigma_{1}-\sigma_{0}\right)^{-d}\|\tilde{\boldsymbol{\Upsilon}}\|_{\left(\sigma_{1}+\sigma_{0}\right) / 2,0}+|\delta \tilde{e}|+|\delta \tilde{m}|+|\delta \tilde{M}| \leq \\
c\left(1+\left|\alpha^{2}\right|\right)\left(\sigma_{1}-\sigma_{0}\right)^{-8 n-13-2 d}\|\boldsymbol{\Phi}\|_{\sigma_{1}, 0}\|\delta \mathfrak{u}\|_{\sigma_{1}, 0} .
\end{gathered}
$$

which obviously leads to (5.14).

Step 3. It remains to prove estimate (5.15). Choose an arbitrary $\mathbf{F} \in$ $\mathcal{F}_{\sigma_{1}, 0}$ and set $\delta \mathfrak{u}:=\mathcal{R}(\mathfrak{f}, \mathfrak{u})[\mathbf{F}]$. It follows from the definition 5.3 that

$$
\delta \mathfrak{u}=(\boldsymbol{\Xi}[\Upsilon \boldsymbol{\Upsilon}], \delta e, \delta m, \delta M),
$$

where $(\boldsymbol{\Upsilon}, \delta e, \delta m, \delta M)$ is a solution to problem (5.4). On the other hand, in view of Corollary 4.8, the vector

$$
D_{\mathfrak{u}} \boldsymbol{\Phi}(\mathfrak{f}, \mathfrak{u}) \mathcal{R}(\mathfrak{f}, \mathfrak{u})[\mathbf{F}] \equiv D_{\mathfrak{u}} \boldsymbol{\Phi}(\mathfrak{f}, \mathfrak{u}) \delta \mathfrak{u}
$$

satisfies identities (4.30). Combining (4.30) and (5.4) we arrive at the identity

$$
D_{\mathfrak{u}} \boldsymbol{\Phi}(\mathfrak{f}, \mathfrak{u}) \mathcal{R}(\mathfrak{f}, \mathfrak{u})[\mathbf{F}]-\mathbf{F}=\left(\Pi_{1}[\mathbf{\Upsilon}], \Pi_{2}[\mathbf{\Upsilon}], \Pi_{3}[\mathbf{\Upsilon}], \Pi_{4}[\mathbf{\Upsilon}], \Pi_{5}[\mathbf{\Upsilon}]\right)
$$

Applying Corollary 5.2 to equations (5.4) we conclude that

$$
\|\Upsilon\|_{\left(\sigma_{0}+\sigma_{1}\right) / 2,0}+|\delta e|+|\delta m|+|\delta M| \leq c\left(1+\left|\alpha^{2}\right|\right)\left(\sigma_{1}-\sigma_{0}\right)^{-8 n-12}\|\mathbf{F}\|_{\sigma_{1}, 0},
$$

which gives

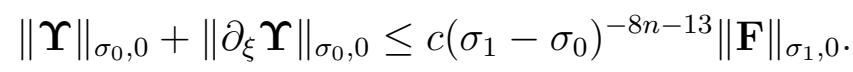


Next we have

$$
\|\boldsymbol{\Phi}\|_{\sigma_{0}, 0}+\left\|\partial_{\xi} \boldsymbol{\Phi}\right\|_{\sigma_{0}, 0} \leq c\left(\sigma_{1}-\sigma_{0}\right)^{-1}\|\boldsymbol{\Phi}\|_{\sigma_{1}, 0} .
$$

Combining estimates (5.33) and (5.34) and recalling formulae (5.26) we arrive at

$$
\|\Pi[\Upsilon]\|_{\sigma_{0}, 0} \leq c\left(\sigma_{1}-\sigma_{0}\right)^{-8 n-14}\left\|\Phi_{i}\right\|_{\sigma_{1}, 0}\|\mathbf{F}\|_{\sigma_{1}, 0} .
$$

It remains to note that desired estimate (5.15) is a straightforward consequence of estimate (5.35) and equality (5.32).

\section{Implicit function theorem}

\subsection{Nash-Moser-Zehnder Implicit Function Theorem}

We prove the local solvability of operator equation (4.16) by using the nashMoser implicit function theorem. There are many different versions of this celebrated theorem. Our considerations are based on the version of the NashMoser implicit function theorem proposed by Zhender, see [15], [24].

Fix $d \geq 2$. Recall Definitions 4.4 and 4.5 of spaces $\mathcal{E}_{\sigma, d}$ and $\mathcal{F}_{\sigma, d}$. Let us consider the abstract operator equation

$$
\boldsymbol{\Phi}(\mathfrak{f}, \mathfrak{u})=0, \quad \mathfrak{f} \in \mathbb{C}^{2}, \quad \mathfrak{u} \in \mathcal{E}_{\sigma, d} .
$$

Here $\boldsymbol{\Phi}$ is a smooth operator. Assume that the operator $\boldsymbol{\Phi}$ satisfies the following conditions, cf. [15], ch.6.1.

For fixed $R>0, N>0$, and $\left(\mathfrak{f}_{0}, \mathfrak{u}_{0}\right) \in \mathbb{C}^{2} \times \mathcal{E}_{1, d}$ denote by $\mathcal{B}_{\sigma}\left(\mathfrak{f}_{0}, \mathfrak{u}_{0}\right) \subset$ $\mathbb{C}^{2} \times \mathcal{E}_{\sigma, d}$ the ball

$$
\mathcal{B}_{\sigma}\left(\mathfrak{f}_{0}, \mathfrak{u}_{0}\right)=\left\{(\mathfrak{f}, \mathfrak{u}):\left|\mathfrak{f}-\mathfrak{f}_{0}\right|<N, \quad\left\|\mathfrak{u}-\mathfrak{u}_{0}\right\|_{\sigma, d}<R\right\} .
$$

H.3 The mapping $\boldsymbol{\Phi}$ is defined in $\mathcal{B}_{0}\left(\mathfrak{f}_{0}, \mathfrak{u}_{0}\right)$. Moreover, the mapping $\boldsymbol{\Phi}$ : $\mathcal{B}_{\sigma}\left(\mathfrak{f}_{0}, \mathfrak{u}_{0}\right) \rightarrow \mathcal{F}_{\sigma, 0}$ is continuous for all $\sigma \in(0,1]$. For every $\sigma^{\prime}<\sigma$, the mapping $\boldsymbol{\Phi}(\mathfrak{f}, \cdot): \mathcal{B}_{\sigma}\left(\mathfrak{f}_{0}, \mathfrak{u}_{0}\right) \cap \mathcal{E}_{\sigma, d} \rightarrow \mathcal{F}_{\sigma^{\prime}, 0}, \sigma^{\prime}<\sigma$ is differentiable. For every $(\mathfrak{f}, \mathfrak{u}) \in \mathcal{B}_{\sigma}\left(\mathfrak{f}_{0}, \mathfrak{u}_{0}\right)$, the quantity

$$
Q(\mathfrak{f} ; \mathfrak{u}, \mathfrak{v})=\boldsymbol{\Phi}(\mathfrak{f}, \mathfrak{u})-\boldsymbol{\Phi}(\mathfrak{f}, \mathfrak{v})-D_{\mathfrak{v}} \Phi(\mathfrak{f}, \mathfrak{v})(\mathfrak{u}-\mathfrak{v})
$$

admits the estimate

$$
\|Q(\mathfrak{f} ; \mathfrak{u}, \mathfrak{v})\|_{\sigma^{\prime}, 0} \leq c_{0}\left(\sigma-\sigma^{\prime}\right)^{-2 \tau}\|\mathfrak{u}-\mathfrak{v}\|_{\sigma, d}^{2} .
$$


H.4 For every $\sigma \in(0,1]$ and $(\mathfrak{f}, \mathfrak{u}),(\mathfrak{g}, \mathfrak{u}) \in \mathcal{B}_{\sigma}\left(\mathfrak{f}_{0}, \mathfrak{u}_{0}\right)$, we have

$$
\|\boldsymbol{\Phi}(\mathfrak{f}, \mathfrak{u})-\boldsymbol{\Phi}(\mathfrak{g}, \mathfrak{u})\|_{\sigma, 0} \leq c_{0}|\mathfrak{f}-\mathfrak{g}|
$$

H.5 For every $\sigma \in(0,1]$, every $0 \leq \sigma^{\prime}<\sigma$, and every $(\mathfrak{f}, \mathfrak{u}), \in \mathcal{B}_{\sigma}\left(\mathfrak{f}_{0}, \mathfrak{u}_{0}\right)$, there exists the linear continuous mapping $\mathcal{R}(\mathfrak{f}, \mathfrak{u}): \mathcal{F}_{\sigma, 0} \rightarrow \mathcal{E}_{\sigma^{\prime}, d}$ such that for all $\mathfrak{h} \in \mathcal{F}_{\sigma, 0}$ and all $\mathfrak{v} \in \mathcal{E}_{\sigma, d}$,

$$
\|\mathcal{R}(\mathfrak{f}, \mathfrak{u}) \mathfrak{h}\|_{\sigma^{\prime}, d} \leq c_{0}\left(\sigma-\sigma^{\prime}\right)^{-\gamma}\|\mathfrak{h}\|_{\sigma, 0}
$$

$$
\begin{aligned}
& \|\left(D_{\mathfrak{u}} \mathbf{\Phi}(\mathfrak{f}, \mathfrak{u}) \mathcal{R}(\mathfrak{f}, \mathfrak{u})-\mathbf{I}\right) \\
& \quad \leq \|_{\sigma^{\prime}, 0} \\
& \quad \leq c_{0}\left(\sigma-\sigma^{\prime}\right)^{-2 \tau-\gamma}\|\boldsymbol{\Phi}(\mathfrak{f}, \mathfrak{u})\|_{\sigma, 0}\|\mathfrak{h}\|_{\sigma, 0} \\
& \begin{aligned}
\|\left(\mathcal{R}(\mathfrak{f}, \mathfrak{u}) D_{\mathfrak{u}} \boldsymbol{\Phi}(\mathfrak{f}, \mathfrak{u})-\mathbf{I}\right) & \mathfrak{v} \|_{\sigma^{\prime}, d} \\
& \leq c_{0}\left(\sigma-\sigma^{\prime}\right)^{-2 \tau-\gamma}\|\boldsymbol{\Phi}(\mathfrak{f}, \mathfrak{u})\|_{\sigma, 0}\|\mathfrak{v}\|_{\sigma, d}
\end{aligned}
\end{aligned}
$$

The following theorem, see [15], Theorem 6.1 and Corollaries, constitute the local existence and uniqueness of solutions to operator equation (6.1)

Theorem 6.1. Assume that $\mathbf{\Phi}$ satisfies Conditions $(\mathbf{H . 3})-(\mathbf{H . 5})$. Then there exists a constant $C$, depending only on $c_{0}, \tau$, and $\gamma$ with the following properties. If for some $\sigma \in(0,1]$, the couple $(\mathfrak{f}, \mathfrak{v}(\mathfrak{f})) \in \mathcal{B}_{\sigma}\left(\mathfrak{f}_{0}, \mathfrak{u}_{0}\right)$ satisfies the conditions

$$
\left\|\mathfrak{v}(\mathfrak{f})-\mathfrak{u}_{0}\right\|_{\sigma, d} \leq r \leq R, \quad\|\mathbf{\Phi}(\mathfrak{f}, \mathfrak{v}(\mathfrak{f}))\|_{\sigma, 0} \leq C(R-r) \sigma^{2(\tau+\gamma)}
$$

then equation (6.1) have a solution $\mathfrak{u}=\mathfrak{u}(\mathfrak{f}) \in \mathcal{E}_{\sigma / 2, d}$ such that

$$
\|\mathfrak{v}(\mathfrak{f})-\mathfrak{u}(\mathfrak{f})\|_{\sigma / 2, d} \leq C^{-1}\|\mathbf{\Phi}(\mathfrak{f}, \mathfrak{v}(\mathfrak{f}))\|_{\sigma, 0} \sigma^{-\gamma} .
$$

Moreover, if the mapping $\mathcal{B}_{\sigma} \cap \mathbb{C}^{2} \ni \mathfrak{f} \rightarrow \mathfrak{v}(\mathfrak{f}) \in \mathcal{E}_{\sigma, d}$ is continuous, then the mapping $\mathcal{B}_{\sigma}\left(\mathfrak{f}_{0}, \mathfrak{u}_{0}\right) \cap \mathbb{C}^{2} \ni \mathfrak{f} \rightarrow \mathfrak{u}(\mathfrak{f}) \in \mathcal{E}_{\sigma / 2, d}$ is continuous. If, in addition, $\mathfrak{v}(\mathfrak{f})$ satisfies the inequality

$$
2 C^{-1} \| \mathbf{\Phi}\left(\mathfrak{f}, \mathfrak{v}(\mathfrak{f}) \|_{\sigma, 0} \sigma^{-\gamma}<1\right.
$$

then the solution $\mathfrak{u}(\mathfrak{f})$ is unique. 
Proof. Existence of solution $\mathfrak{u}(\mathfrak{f})$ is exactly the statement Theorem 6.1 in [15]. The continuity of this solution is a consequence of the Corollary of this theorem, see [15]. The uniqueness also results from ch.6 in[15]. However, since our formulation is slightly different, we recall these arguments. Let $\mathfrak{u}(f), \mathfrak{u}^{\prime}(\mathfrak{f}) \in \mathcal{B}_{\sigma}\left(\mathfrak{f}_{0}, \mathfrak{u}_{0}\right)$ satisfy equation (6.1) and inequalities (6.9). It follows that

$$
D_{\mathfrak{u}} \Phi(\mathfrak{f}, \mathfrak{u}(\mathfrak{f}))\left(\mathfrak{u}^{\prime}(\mathfrak{f})-\mathfrak{u}(\mathfrak{f})\right)=-Q\left(\mathfrak{f}, \mathfrak{u}, \mathfrak{u}^{\prime}\right)
$$

Applying to both sides of this identity the operator $\mathcal{R}(\mathfrak{f}, \mathfrak{u}(\mathfrak{f})$ and recalling that $\mathfrak{u}(\mathfrak{f})$ satisfies equation (6.1) we obtain

$$
\mathfrak{u}^{\prime}(\mathfrak{f})-\mathfrak{u}(\mathfrak{f})=-\mathcal{R}\left(\mathfrak{f}, \mathfrak{u}(\mathfrak{f}) Q\left(\mathfrak{f}, \mathfrak{u}, \mathfrak{u}^{\prime}\right) .\right.
$$

Choose an arbitrary $\sigma^{\prime \prime}<\sigma^{\prime} \leq \sigma$.Applying inequalities (6.3) and (6.5) we arrive at

$$
\begin{aligned}
\left\|\mathfrak{u}^{\prime}(\mathfrak{f})-\mathfrak{u}(\mathfrak{f})\right\|_{\sigma^{\prime \prime}, d} \leq c\left(\sigma^{\prime}-\sigma^{\prime \prime}\right)^{-\gamma}\left\|Q\left(\mathfrak{f}, \mathfrak{u}, \mathfrak{u}^{\prime}\right)\right\|_{\left(\sigma^{\prime}+\sigma^{\prime \prime}\right) / 2, d} \leq \\
c\left(\sigma^{\prime}-\sigma^{\prime \prime}\right)^{-2 \tau-\gamma}\left\|\mathfrak{u}^{\prime}(\mathfrak{f})-\mathfrak{u}(\mathfrak{f})\right\|_{\sigma^{\prime \prime}, d}^{2}
\end{aligned}
$$

Now set $\sigma_{n}=2^{-n-1} \sigma, n \geq 0$. We have

$$
\left\|\mathfrak{u}^{\prime}(\mathfrak{f})-\mathfrak{u}(\mathfrak{f})\right\|_{\sigma_{n+1}, d} \leq c 2^{n(\gamma+2 \tau)}\left\|\mathfrak{u}^{\prime}(\mathfrak{f})-\mathfrak{u}(\mathfrak{f})\right\|_{\sigma_{n}, d}
$$

which leads to the inequality

$$
\left\|\mathfrak{u}^{\prime}(\mathfrak{f})-\mathfrak{u}(\mathfrak{f})\right\|_{\sigma_{n+1}, d} \leq c^{n} 2^{n^{2}(\gamma+2 \tau)}\left\|\mathfrak{u}^{\prime}(\mathfrak{f})-\mathfrak{u}(\mathfrak{f})\right\|_{\sigma / 2, d}^{2^{n}},
$$

On the other hand, estimate (6.9) imply

$$
\left\|\mathfrak{u}^{\prime}(\mathfrak{f})-\mathfrak{u}(\mathfrak{f})\right\|_{\sigma / 2, d} \leq 2 C^{-1}\|\boldsymbol{\Phi}(\mathfrak{f}, \mathfrak{v}(\mathfrak{f}))\|_{\sigma, 0} \sigma^{-\gamma} .
$$

Combining the obtained results and recalling (6.10) we finally obtain

$$
\begin{aligned}
\left\|\mathfrak{u}^{\prime}(\mathfrak{f})-\mathfrak{u}(\mathfrak{f})\right\|_{0, d} \leq & \left\|\mathfrak{u}^{\prime}(\mathfrak{f})-\mathfrak{u}(\mathfrak{f})\right\|_{\sigma_{n+1}, d} \leq \\
& c^{n} 2^{n^{2}(\gamma+2 \tau)}\left(2 C^{-1}\|\boldsymbol{\Phi}(\mathfrak{f}, \mathfrak{v}(\mathfrak{f}))\|_{\sigma, 0} \sigma^{-\gamma}\right)^{2^{n}} \rightarrow 0 \text { as } n \rightarrow \infty .
\end{aligned}
$$




\subsection{Solvability of the main operator equation}

We are now in a position to prove the local solvability and uniqueness result for the main operator equation (4.16). Recall the notation

$$
\mathfrak{f}=(\alpha, k), \mathfrak{u}=(\boldsymbol{\varphi}, e, m, M) \text { where } \boldsymbol{\varphi}=\left(\boldsymbol{\beta}, \varphi_{0}, \mathbf{u}, \mathbf{w}, W_{11}, W_{12}, W_{22}\right) .
$$

and

$$
\mathfrak{v}(\mathfrak{f})=\left(\boldsymbol{\varphi}_{0}(\alpha),-k \alpha-k \alpha^{2} / 2, k \alpha,-k\right),
$$

where

$$
\boldsymbol{\varphi}_{0}(\alpha)=\left(0,0, \alpha \mathbf{e}_{1}, 1,0,0\right) .
$$

Fix an arbitrary $\sigma \in(0,1]$ and $d \geq 1$. Next, fix $\varrho>0$ satisfying Condition (H.1) of Theorem 2.2 and set

$$
G=\left\{(\alpha, k) \in \mathbb{C}^{2}:|\operatorname{Re} \alpha| \leq 4 \pi, \operatorname{Re} k \in[0,1], \quad|\operatorname{Im} \alpha| \leq \varrho,|\operatorname{Im} k| \leq \varrho\right\} .
$$

The following theorem is the main result of this section

Theorem 6.2. Let conditions $(\mathbf{H} .1)-\left(\right.$ H.2) be satisfied. Then there is $\varepsilon_{0}>0$ such that for all $|\varepsilon| \leq \varepsilon_{0}$ and for all $\mathfrak{f} \in G$ operator equation (4.16) has a unique solution $\mathfrak{u}(\mathfrak{f}) \in \mathcal{E}_{\sigma / 2, d}$ such that

$$
\|\mathfrak{u}(\mathfrak{f})-\mathfrak{v}(\mathfrak{f})\|_{\sigma / 2, d} \leq c|\varepsilon| .
$$

The mapping $G \ni \mathfrak{f} \rightarrow \mathfrak{u}(\mathfrak{f}) \in \mathcal{E}_{\sigma / 2, d}$ has a continuous extension to the strip

$$
\Sigma_{\rho}=\left\{(\alpha, k) \in \mathbb{C}^{2}: \operatorname{Re} \alpha \in \mathbb{R}, \operatorname{Re} k \in[0,1], \quad|\operatorname{Im} \alpha| \leq \varrho,|\operatorname{Im} k| \leq \varrho\right\} .
$$

Moreover, the extended mappings

$$
\mathfrak{f} \rightarrow \boldsymbol{\varphi}-\varphi_{0}(\alpha), \quad \mathfrak{f} \rightarrow M, \quad \mathfrak{f} \rightarrow m+\alpha M
$$

are $2 \pi$ - periodic in $\alpha$.

Proof. In view of Proposition 4.6 for every $\mathfrak{f} \in G$, there is $r>0$ independent of $\mathfrak{f}$ with the following property. If

$$
\|\mathfrak{v}(\mathfrak{f})-\mathfrak{u}\|_{\sigma, d} \leq r
$$

then the operator $\Phi(\mathfrak{f}, \mathfrak{u}) \in \mathcal{F}_{\sigma, 0}$ is differentiable at the point $(\mathfrak{f}, \mathfrak{u})$ with respect to $\mathfrak{u}$ and satisfies estimate (6.3) with the exponent $\tau=0$. On the other 
hand, it follows from Proposition 5.4, that there exists $\varepsilon_{0}>0$ independent of $\mathfrak{f}$ with the following properties. If $\mathfrak{f} \in G, \mathfrak{u} \in \mathcal{F}_{\sigma, 0}$ satisfy inequality (6.15), and $|\varepsilon| \leq \varepsilon_{0}$, then there is the linear operator $\mathcal{R}(\mathfrak{f}, \mathfrak{u})$ satisfying inequalities (6.5)-(6.7) with the exponents $\tau=0$ and $\gamma=8 n+14+2 d$. In other words, if $(\mathfrak{f}, \mathfrak{u})$ satisfies inequality (6.15) and $|\varepsilon| \leq \varepsilon_{0}$, then the operator $\Phi$ satisfies inequalities (6.3) and (6.5)-(6.7) in Conditions (H.3)-(H.5) of Theorem 6.1.

Now choose an arbitrary $\mathfrak{f}_{0}=\left(\alpha_{0}, k_{0}\right) \in G$ and set $\mathfrak{u}_{0}=\mathfrak{v}\left(\mathfrak{f}_{0}\right)$. It follows from this and (6.12) that

$$
\left\|\mathfrak{v}(\mathfrak{f})-\mathfrak{u}_{0}\right\|_{\sigma, d} \leq 20\left|\mathfrak{f}-\mathfrak{f}_{0}\right| \text { for all } \mathfrak{f} \in G .
$$

Recall definition $(\underline{6.2})$ of the ball $\mathcal{B}_{\sigma}\left(\mathfrak{f}_{0}, \mathfrak{u}_{0}\right)$ :

$$
\mathcal{B}_{\sigma}\left(\mathfrak{f}_{0}, \mathfrak{u}_{0}\right)=\left\{(\mathfrak{f}, \mathfrak{u}):\left|\mathfrak{f}-\mathfrak{f}_{0}\right|<N, \quad\left\|\mathfrak{u}-\mathfrak{u}_{0}\right\|_{\sigma, d}<R\right\} .
$$

We have

$$
\begin{aligned}
\|\mathfrak{v}(\mathfrak{f})-\mathfrak{u}\|_{\sigma, d} \leq & \left\|\mathfrak{u}_{0}-\mathfrak{u}\right\|_{\sigma, d}+\left\|\mathfrak{v}(\mathfrak{f})-\mathfrak{u}_{0}\right\|_{\sigma, d} \\
& \leq R+20\left|\mathfrak{f}-\mathfrak{f}_{0}\right| \leq R+20 N
\end{aligned}
$$

for $(\mathfrak{f}, \mathfrak{u}) \in \mathcal{B}_{\sigma}$. Hence for

$$
R+20 N \leq r, \quad N<\rho
$$

the vectors $\mathfrak{f}$ and $\mathfrak{u}$ satisfy inequality (6.15) in the ball $\mathcal{B}_{\sigma}\left(\mathfrak{f}_{0}, \mathfrak{u}_{0}\right)$. Moreover, we have $\mathcal{B}_{\sigma}\left(\mathfrak{f}_{0}, \mathfrak{u}_{0}\right) \cap \mathbb{C}^{2} \subset \Sigma_{\rho}$. Now fix $N$ and $R$ satisfying (6.17). It follows from this and what was mentioned above that for $|\varepsilon| \leq \varepsilon_{0}$, the operator $\Phi$ satisfies Conditions (H.3)-(H.5) with the exponents $\tau=0, \gamma=9 n+12+d$, and the constant $c_{0}$ independent of $\mathfrak{f}_{0}$. Hence $\Phi$ meets all requirements of Theorem 6.1. Applying this theorem we conclude that there is a constant $C$, depending only on $c_{0}$ and $\gamma$, with the following properties. If the couple $(\mathfrak{f}, \mathfrak{v}(\mathfrak{f})) \in \mathcal{B}_{\sigma}\left(\mathfrak{f}_{0}, \mathfrak{u}_{0}\right)$ satisfies the conditions

$$
\left\|\mathfrak{v}(\mathfrak{f})-\mathfrak{u}_{0}\right\|_{\sigma, d} \leq R / 2, \quad\|\boldsymbol{\Phi}(\mathfrak{f}, \mathfrak{v}(\mathfrak{f}))\|_{\sigma, 0} \leq 2^{-1} C R \sigma^{2 \gamma},
$$

then equation (6.1) have a unique solution $\mathfrak{u}=\mathfrak{u}(\mathfrak{f}) \in \mathcal{E}_{\sigma / 2, d}$ such that

$$
\|\mathfrak{v}(\mathfrak{f})-\mathfrak{u}(\mathfrak{f})\|_{\sigma / 2, d} \leq C^{-1}\|\boldsymbol{\Phi}(\mathfrak{f}, \mathfrak{v}(\mathfrak{f}))\|_{\sigma, 0} \sigma^{-\gamma} .
$$

Moreover, the mapping $\mathcal{B}_{\sigma}\left(\mathfrak{f}_{0}, \mathfrak{u}_{0}\right) \cap \mathbb{C}^{2} \ni \mathfrak{f} \rightarrow \mathfrak{u}(\mathfrak{f}) \in \mathcal{E}_{\sigma / 2, d}$ is continuous. In order to prove the local existence and uniqueness of solution to equation 
(4.16), we have to show that conditions (6.18) is fulfilled in the ball $\mathcal{B}_{\sigma}\left(\mathfrak{f}_{0}, \mathfrak{u}_{0}\right)$. First we notice that, in view of relations (6.12) and (4.9), we have

$$
\begin{gathered}
\Phi_{1}(\mathfrak{f}, \mathfrak{v}(\mathfrak{f}))=\varepsilon H_{1}\left(\mathrm{id}, 0, \alpha \mathbf{e}_{1}\right), \\
\left.\Phi_{2}(\mathfrak{f}, \mathfrak{v}(\mathfrak{f}))=\varepsilon \frac{\partial H_{1}}{\partial \mathbf{z}}\left(\mathrm{id}, 0, \alpha \mathbf{e}_{1}\right)\right\}^{\top}, \\
\Phi_{3}(\mathfrak{f}, \mathfrak{v}(\mathfrak{f}))=\varepsilon\left\{\frac{\partial H_{1}}{\partial \mathbf{y}}\left(\mathrm{id}, 0, \alpha \mathbf{e}_{1}\right)\right\}^{\top}, \\
\Phi_{4}(\mathfrak{f}, \mathfrak{v}(\mathfrak{f}))=\varepsilon \frac{\partial^{2} H_{1}}{\partial \mathbf{z}^{2}}\left(\mathrm{id}, 0, \alpha \mathbf{e}_{1}\right), \\
\Phi_{5}(\mathfrak{f}, \mathfrak{v}(\mathfrak{f}))=0 .
\end{gathered}
$$

It follows from this and analyticity conditions (2.3) imposed on $H_{1}$ that

$$
\|\Phi(\mathfrak{f}, \mathfrak{v}(f))\|_{\sigma, 0} \leq c_{1}|\varepsilon|
$$

for all $\mathfrak{f} \in \Sigma_{\rho}$. Here the constant $c_{1}$ is independent on $\mathfrak{f}$. From this and (6.16) we obtain that condition (6.18) is fulfilled for all $\mathfrak{f}$ satisfying the inequality

$$
\left|\mathfrak{f}-\mathfrak{f}_{0}\right| \leq N_{1}=N / 40,
$$

and all $\varepsilon$ such that

$$
\varepsilon \leq \varepsilon_{0}<c_{1}^{-1} C N \sigma^{2 \gamma} .
$$

Applying Theorem 6.1 we conclude that for all $\mathfrak{f} \in G$ satisfying (6.20) and for all $|\varepsilon| \leq \varepsilon_{0}$ operator equation (4.16) has a solution $\mathfrak{u}(\mathfrak{f})$ satisfying the inequality

$$
\|\mathfrak{v}(\mathfrak{f})-\mathfrak{u}(\mathfrak{f})\|_{\sigma / 2, d} \leq c_{1} C^{-1} \sigma^{-\gamma}|\varepsilon| \equiv c_{2}|\varepsilon| .
$$

This solution is unique. Moreover, the mapping $\mathbb{C}^{2} \ni \mathfrak{f} \rightarrow \mathfrak{u}(\mathfrak{f}) \in \mathcal{E}_{\sigma / 2, d}$ is continuous in the disk $\left\{\left|\mathfrak{f}-\mathfrak{f}_{0}\right| \leq N_{1}\right\}$.

Our next task is to prove that this solution is defined for all $\mathfrak{f} \in G$. Since $G$ is a compact set, there is a finite collection of the balls

$$
G_{i}=\left\{\left|\mathfrak{f}-\mathfrak{f}_{i}\right| \leq N_{1} / 2\right\}, \quad 1 \leq i \leq m, \quad \mathfrak{f}_{i} \in G
$$

such that $G \subset \cup_{i} G_{i}$. It follows from the local solvability of operator equation (4.16) that for $|\varepsilon| \leq \varepsilon_{0}$ there is a unique continuous mapping $G_{i} \ni \mathfrak{f} \rightarrow \mathfrak{u}_{i}(\mathfrak{f}) \in$ $\mathcal{E}_{\sigma / 2, d}$ such that

$$
\Phi\left(\mathfrak{f}, \mathfrak{u}_{i}(\mathfrak{f})\right)=0, \quad\left\|\mathfrak{u}_{i}(\mathfrak{f})-\mathfrak{v}(\mathfrak{f})\right\|_{\sigma / 2, d} \leq c_{2}|\varepsilon|
$$


If $\mathfrak{f} \in G_{i} \cap G_{j}$ then $\mathfrak{u}_{i}(\mathfrak{f})=\mathfrak{u}_{j}(\mathfrak{f})$. Indeed, $\mathfrak{u}_{j}$ satisfies inequality (6.22) and the equation $\Phi\left(\mathfrak{f}, \mathfrak{u}_{j}\right)=0$. On the other hand, $\mathfrak{u}_{i}(\mathfrak{f})$ is the unique solution to the equation $\Phi(\mathfrak{f}, \mathfrak{u})=0$ with $\mathfrak{f} \in G_{i}$ satisfying this inequality. Hence $\mathfrak{u}_{i}=\mathfrak{u}_{j}$. Therefore, the relation $\mathfrak{u}(\mathfrak{f})=\mathfrak{u}_{i}(\mathfrak{f})$ for $\mathfrak{f} \in G_{i}$ defines the continuous mapping $G \ni \mathfrak{f} \rightarrow \mathfrak{u}(\mathfrak{f}) \in \mathcal{E}_{\sigma / 2, d}$

It remains to prove that this mapping has the analytic extension to the strip $\Sigma_{\rho}$. Fix an arbitrary $\mathfrak{f}=(\alpha, k) \in G$ such that

$$
\operatorname{Re} \alpha \in[0, \pi / 2]
$$

and set

$$
\tilde{\alpha}=\alpha+2 \pi, \quad \tilde{\mathfrak{f}}=(\tilde{\alpha}, k) .
$$

Let $\tilde{\mathfrak{u}}=\mathfrak{u}(\tilde{\mathfrak{f}})$ be a solution to the operator equation $\Phi(\tilde{\mathfrak{f}}, \tilde{\mathfrak{u}})=0$. We have

$$
\tilde{\mathfrak{u}}=(\tilde{\boldsymbol{\varphi}}, \tilde{e}, \tilde{m}, \tilde{M}) \text {, where } \tilde{\boldsymbol{\varphi}}=\left(\tilde{\boldsymbol{\beta}}, \tilde{\varphi}_{0}, \tilde{\mathbf{u}}, \tilde{\mathbf{w}}, \tilde{W}_{11}, \tilde{W}_{12}, \tilde{W}_{22}\right) \text {. }
$$

Now set

$$
\mathfrak{u}^{*}=\left(\varphi^{*}, e^{*}, m^{*}, M^{*}\right)
$$

where

$$
\begin{array}{r}
\boldsymbol{\varphi}^{*}=\tilde{\boldsymbol{\varphi}}-\left(0,0,0,2 \pi \mathbf{e}_{1}, 0,0,0\right), \\
e^{*}=\tilde{e}-\left(2 \pi \tilde{m}+2 \pi^{2} \tilde{M}\right), \quad m^{*}=\tilde{m}+2 \pi \tilde{M}, \quad M^{*}=\tilde{M} .
\end{array}
$$

Since the basic Hamiltonian $H(\mathbf{x}, \mathbf{y}, \mathbf{z})$ is $2 \pi$-periodic in $z_{1}$ it follows from definition (4.9) of the operator $\Phi$ that

$$
\Phi\left(\mathfrak{f}, \mathfrak{u}^{*}\right) \equiv \Phi(\tilde{\mathfrak{f}}, \tilde{\mathfrak{u}})
$$

Next, we have

$$
\mathfrak{v}(\tilde{\mathfrak{f}})=\mathfrak{v}(\mathfrak{f})-\left(2 \pi k+2 \pi^{2} k,-2 \pi k, k\right) .
$$

Relations (6.24) imply

$$
\mathfrak{u}^{*}-\mathfrak{v}(\mathfrak{f})=\tilde{\mathfrak{u}}-\mathfrak{v}(\tilde{\mathfrak{f}})
$$

Recall that $\tilde{\mathfrak{u}}=\mathfrak{u}(\tilde{\mathfrak{f}})$ satisfies the inequality

$$
\|\tilde{\mathfrak{u}}-\mathfrak{v}(\tilde{\mathfrak{f}})\|_{\sigma / 2, d} \leq c_{2}|\varepsilon| .
$$


and $\Phi(\tilde{\mathfrak{f}}, \tilde{\mathfrak{u}})=0$. From this, $\underline{6.25}$ and $(\underline{6.26}$ ) we obtain

$$
\Phi\left(\mathfrak{f}, \mathfrak{u}^{*}\right)=0, \quad\left\|\mathfrak{u}^{*}-\mathfrak{v}(\mathfrak{f})\right\|_{\sigma / 2, d} \leq c_{2}|\varepsilon|, \quad \mathfrak{f} \in G
$$

Hence $\mathfrak{u}^{*}=\mathfrak{u}(\mathfrak{f})$, which along with $(6.24)$ and the equality $\tilde{\mathfrak{u}}=\mathfrak{u}(\tilde{\mathfrak{f}})$ yields

$$
\begin{array}{r}
\boldsymbol{\varphi}(\mathfrak{f})+2 \pi\left(0,0,0, \mathbf{e}_{1}, 0,0,0\right)=\boldsymbol{\varphi}(\tilde{\mathfrak{f}}), \\
m(\mathfrak{f})=m(\tilde{\mathfrak{f}})+2 \pi M(\tilde{\mathfrak{f}}), \quad M(\mathfrak{f})=M(\tilde{\mathfrak{f}}) .
\end{array}
$$

We can rewrite these relations in the equivalent form

$$
\begin{array}{r}
\boldsymbol{\varphi}(\mathfrak{f})-\alpha\left(0,0,0, \mathbf{e}_{1}, 0,0,0\right)=\boldsymbol{\varphi}(\tilde{\mathfrak{f}})-(\alpha+2 \pi)\left(0,0,0, \mathbf{e}_{1}, 0,0,0\right), \\
m(\mathfrak{f})+\alpha M(\mathfrak{f})=m(\tilde{\mathfrak{f}})+(\alpha+2 \pi) M(\tilde{\mathfrak{f}}), \quad M(\mathfrak{f})=M(\tilde{\mathfrak{f}}) .
\end{array}
$$

Recall that these relations holds true for all $\mathfrak{f}$ and $\tilde{\mathfrak{f}}$ satisfying the conditions

$$
\mathfrak{f}=(\alpha, k) \in G, \quad \tilde{\mathfrak{f}}=(\alpha+2 \pi, k), \quad \operatorname{Re} \alpha \in[0, \pi / 2] .
$$

Since the vector-function $\mathfrak{u}(\mathfrak{f})$ and all its components are holomorphic in $\alpha$, relations (6.27) imply that the mappings

$$
\mathfrak{f} \rightarrow \boldsymbol{\varphi}-\boldsymbol{\varphi}_{0}(\alpha), \quad \mathfrak{f} \rightarrow M, \quad \mathfrak{f} \rightarrow m+\alpha M
$$

are holomorphic in the strip $\Sigma_{\rho}$ and $2 \pi$ periodic in $\alpha$. This completes the proof of Theorem 6.2 .

\section{Dependence on parameters. Jacobi vector fields}

\subsection{Differentiability with respect to parameters}

Theorem 6.2 guarantees the local existence of solutions to the operator equation

$$
\Phi(\mathfrak{f}, \mathfrak{u})=0 .
$$

In this section we investigate in details the dependence of this solution on the parameter $\mathfrak{f}$. The result is given by the following 
Theorem 7.1. Let conditions $(\mathbf{H . 1})-(\mathbf{H} .2)$ be satisfied and $\varepsilon_{0}>0$ be given by Theorem 6.2. Let $|\varepsilon| \leq \varepsilon_{0}$ and $\mathfrak{u}=\mathfrak{u}(\mathfrak{f})$ be a solution to equation (7.1),

$$
\begin{array}{r}
\mathfrak{u}(\mathfrak{f})=(\boldsymbol{\varphi}(\alpha, k), e(\alpha, k), m(\alpha, k), M(\alpha, k), \\
\boldsymbol{\varphi}(\alpha, k)=\left(\boldsymbol{\beta}, \varphi_{0}, \mathbf{u}, \mathbf{w}, W_{11}, W_{12}, W_{21}\right) .
\end{array}
$$

Then the mappings

$$
\begin{array}{r}
\mathbb{R} \times[0,1] \ni(\alpha, k) \rightarrow \mathbf{u}(\alpha, k) \in \mathcal{A}_{\sigma / 4, d}, \\
\mathbb{R} \times[0,1] \ni(\alpha, k) \rightarrow \varphi_{0}(\alpha, k) \in X_{\sigma / 4, d}, \\
\mathbb{R} \times[0,1] \ni(\alpha, k) \rightarrow \mathbf{w}(\alpha, k)-\alpha \mathbf{e}_{1} \in \mathcal{A}_{\sigma / 2, d}, \\
\mathbb{R} \times[0,1] \ni(\alpha, k) \rightarrow \mathbf{W}(\alpha, k) \in \mathcal{A}_{\sigma / 2, d}, \\
\mathbb{R} \times[0,1] \ni(\alpha, k) \rightarrow \boldsymbol{\beta}(\alpha, k) \in \mathbb{R}^{n-1}, \\
\mathbb{R} \times[0,1] \ni(\alpha, k) \rightarrow M(\alpha, k) \in \mathbb{R}^{1}, \\
\mathbb{R} \times[0,1] \ni(\alpha, k) \rightarrow m(\alpha, k)+\alpha M(\alpha, k) \in \mathbb{R}^{1},
\end{array}
$$

are continuously differentiable and $2 \pi$-periodic in $\alpha$. Moreover, they are analytic in $\mathbb{R} \times(0,1)$ and satisfy the inequalities

$$
\begin{aligned}
\left\|\partial_{\alpha}^{r} \mathbf{u}\right\|_{\sigma / 2, d}+\left\|\partial_{\alpha}^{r} \varphi_{0}\right\|_{\sigma / 2, d} & \leq c(r)|\varepsilon|, \\
\left\|\partial_{\alpha}^{r}\left(\mathbf{w}-\alpha \mathbf{e}_{1}\right)\right\|_{\sigma / 2, d}+\left\|\partial_{\alpha}^{r}(\mathbf{W}-\mathbf{I})\right\|_{\sigma / 2, d} & \leq c(r)|\varepsilon|, \\
\left|\partial_{\alpha}^{r} \boldsymbol{\beta}\right|+\left|\partial_{\alpha}^{r}(m+\alpha M)\right|+\left|\partial_{\alpha}^{r}(M+k)\right| & \leq c(r)|\varepsilon|,
\end{aligned}
$$

and

$$
\begin{aligned}
\left\|\partial_{k} \mathbf{u}\right\|_{\sigma / 2, d}+\left\|\partial_{k} \varphi_{0}\right\|_{\sigma / 2, d} & \leq c|\varepsilon|, \\
\left\|\partial_{k}\left(\mathbf{w}-\alpha \mathbf{e}_{1}\right)\right\|_{\sigma / 2, d}+\left\|\partial_{k}(\mathbf{W}-\mathbf{I})\right\|_{\sigma / 2, d} & \leq c|\varepsilon|, \\
\left|\partial_{k} \boldsymbol{\beta}\right|+\left|\partial_{k}(m+\alpha M)\right|+\left|\partial_{k}(M+k)\right| & \leq c|\varepsilon|,
\end{aligned}
$$

where $r \geq 0$ is an arbitrary integer, the constant $c$ is independent of $\alpha, k$, and $\varepsilon$.

Proof. By virtue of Theorem (6.2), the mapping $\Sigma_{\rho} \ni \mathfrak{f} \rightarrow \mathfrak{u}(\mathfrak{f}) \in \mathcal{E}_{\sigma / 2,0}$ is continuous in the complex strip $\Sigma_{\rho}$ which contains the real axis. Hence this mapping is holomorphic function of $\alpha$. It is also a holomorphic function of $k$ on the interval $(0,1)$. Moreover, Theorem 6.2 shows that $\boldsymbol{\beta}, \varphi_{0}, \mathbf{u}, \mathbf{w}-\alpha \mathbf{e}_{1}$, $W_{i j}, m+\alpha M$ and $M$ are periodic in $\alpha$. Since these functions are holomorphic in $\alpha$ on the real axis, estimates (7.4) obviously follows from estimates (6.14) in Theorem 6.2. 
However, this theorem does not guarantee the differentiability of $\mathfrak{u}(\alpha, k)$ with respect to $k$ on the closed segment $k \in[0,1]$. In order to prove estimates (7.5) fix an arbitrary $\alpha \in \mathbb{R}^{1}$ and consider the function $\mathfrak{u}(\alpha, k)$. In view of Theorem 6.2 this function is continuous in the rectangular $\operatorname{Re} k \in[0,1]$, $|\operatorname{Im} k| \leq \rho$. Hence it is holomorphic on the interval $(0,1)$ and continuous on $[0,1]$. Next the function $\mathfrak{u}(\alpha, k)$ satisfies the operator equation

$$
\Phi(\mathfrak{f}, \mathfrak{u}) \equiv\left(\Phi_{1}(\mathfrak{u}), \Phi_{2}(\mathfrak{u}), \Phi_{3}(\mathfrak{u}), \Phi_{4}(k, \mathfrak{u}), \Phi_{5}(\mathfrak{u})\right)=0
$$

where the differential operators $\Phi_{i}$ are defined by (4.9). It follows from this relation that the only $\Phi_{4}$ depends on $k$ via the matrix $\boldsymbol{\Omega}=\operatorname{diag}(-k, 1)$ in the left hand side of (4.9d). Since $\mathfrak{u}$ is analytic function of $k$ on the interval $(0,1)$, we can differentiate (7.6) with respect to $k$ to obtain

$$
\begin{aligned}
& D_{\varphi} \Phi(\mathfrak{f}, \mathfrak{u}) \partial_{k} \boldsymbol{\varphi}+D_{e} \Phi(\mathfrak{f}, \mathfrak{u}) \partial_{k} e+ \\
& \quad D_{m} \Phi(\mathfrak{f}, \mathfrak{u}) \partial_{k} m+D_{M} \Phi(\mathfrak{f}, \mathfrak{u})\left(\partial_{k} M+1\right)=\mathcal{Z} \text { for } k \in(0,1) .
\end{aligned}
$$

Here

$$
\mathcal{Z}=\left(0,0,0, \mathcal{Z}_{4}, 0\right), \quad \mathcal{Z}_{4}=\operatorname{diag}(1,0)-\mathbf{W}^{\top} \operatorname{diag}(1,0) \mathbf{W} .
$$

It follows from estimate (7.4) that

$$
\|\mathcal{Z}\|_{\sigma / 2,0} \leq c|\varepsilon| .
$$

Relation (7.7) can be considered as equation for

$$
\partial_{k} \mathfrak{u}=\left(\partial_{k} \boldsymbol{\varphi}, \partial_{k} e, \partial_{k} m, \partial_{k} M\right) .
$$

Applying to (7.7) Proposition 5.4 with $\sigma, \sigma_{1}$, and $\sigma^{\prime}$ replaced by $\sigma / 2, \sigma / 2$, and $\sigma / 4$ we conclude that for a suitable choice of $\varepsilon_{0}$, equation (7.7) has a unique solution

$$
\left(\partial_{k} \boldsymbol{\varphi}, \partial_{k} e, \partial_{k} m, \partial_{k} M+1\right)=\mathcal{R}(\mathfrak{f}, \mathfrak{u}) \mathcal{Z}
$$

Estimate (5.13) for the resolvent $\mathcal{R}$ in Proposition (5.4) implies the inequality

$$
\left\|\partial_{k} \varphi\right\|_{\sigma / 4, d}+\left|\partial_{k} m\right|+\left|\partial_{k} M+1\right| \leq c \sigma^{-9 n-12-d}\|\mathcal{Z}\|_{\sigma / 2,0} \leq c|\varepsilon|,
$$

which obviously yields (7.5). This completes the proof of Theorem 7.1 . 


\subsection{Representation of derivatives. Jacobi vector fields}

In this section we obtain the representation for the derivatives of solutions to the modified problem with respect to the parameters $\alpha$ and $k$. Let $\boldsymbol{\varphi}(\alpha, k)=$ $\left(\boldsymbol{\beta}, \varphi_{0}, \mathbf{u}, \mathbf{w}, W_{11}, W_{12}, W_{21}\right)$ be a solution to the operator equation (17.1) given by Theorem $\mathbf{7 . 1}$. Let $\mathbf{V}, \mathbf{W}$, and $\mathbf{v}$ are given by (3.4)

$$
\begin{array}{r}
\mathbf{V}=\left(\mathbf{I}_{n-1}+\mathbf{u}^{\prime}\right)^{-\top}, \quad \mathbf{v}=\beta+\mathbf{V}\left(\nabla \varphi_{0}-w_{2} \nabla w_{1}\right) \\
W_{22}=W_{11}^{-1}\left(1+W_{12} W_{21}\right) .
\end{array}
$$

For $\tau=\alpha, k$ denote by $\boldsymbol{\chi}^{(\tau)}, \boldsymbol{\lambda}^{(\tau)}$, and $\boldsymbol{\mu}^{(\tau)}$ the functions

$$
\begin{gathered}
\boldsymbol{\chi}^{(\tau)}=\mathbf{V}^{\top} \partial_{\tau} \mathbf{u} \\
\boldsymbol{\lambda}^{(\tau)}=\mathbf{W}^{-1} \partial_{\tau} \mathbf{w}-\chi_{i}^{(\tau)} \mathbf{W}^{-1} \frac{\partial}{\partial \xi_{i}} \mathbf{w} \\
\boldsymbol{\mu}^{(\tau)}=\mathbf{V}^{-1}\left(\partial_{\tau} \mathbf{v}+\chi_{i}^{(\tau)} \frac{\partial}{\partial \xi_{i}} \mathbf{v}-\boldsymbol{\Lambda} \boldsymbol{\lambda}^{(\tau)}\right) .
\end{gathered}
$$

The vector fields $\boldsymbol{\chi}^{(\tau)}, \boldsymbol{\lambda}^{(\tau)}$, and $\boldsymbol{\mu}^{(\tau)}$ can be regarded as the Jacobi vector fields for the invariant tori problem. We also set

$$
p^{(\tau)}=\partial_{\tau} m+\alpha \partial_{\tau} M
$$

Here the matrix $\boldsymbol{\Lambda}$ are given by (3.8):

$$
\Lambda=-\mathbf{V}\left(\mathbf{w}^{\prime}\right) \mathbf{J} \mathbf{W} .
$$

Throughout of this an the next sections we will use the following notation. For every integrable periodic function $f(\xi)$ we set

$$
\bar{f}=(2 \pi)^{1-n} \int_{\mathbb{T}^{n-1}} f(\xi) d \xi, \quad f^{*}=f-\bar{f} .
$$

We have the following

Lemma 7.2. Under the assumptions of Theorem 17.1, the functions $\boldsymbol{\chi}^{(\tau)}$, $\boldsymbol{\lambda}^{(\tau)}, \boldsymbol{\mu}^{(\tau)}$, and the parameter $q^{(\tau)}$ satisfy the equations

$$
\begin{gathered}
\partial \boldsymbol{\mu}^{(\tau)}=-p^{(\tau)} \nabla w_{1}-\frac{1}{2} \partial_{\tau} M \nabla\left(w_{1}^{*}\right)^{2} \\
\partial \boldsymbol{\lambda}^{(\tau)}+\boldsymbol{\Omega} \boldsymbol{\lambda}^{(\tau)}+\mathbf{T} \boldsymbol{\mu}^{(\tau)}+p^{(\tau)} \mathbf{W}^{\top} \boldsymbol{e}_{1}=-\partial_{\tau} M w_{1}^{*} \mathbf{W}^{\top} \boldsymbol{e}_{1} \\
-\partial \boldsymbol{\lambda}^{(\tau)}+\mathbf{S} \boldsymbol{\mu}^{(\tau)}+\mathbf{T}^{\top} \boldsymbol{\lambda}^{(\tau)}=0
\end{gathered}
$$


and the orthogonality conditions

$$
\begin{gathered}
\overline{\left\{W \boldsymbol{\lambda}^{(\tau)} \cdot \mathbf{e}_{1}\right\}}+\overline{\left\{\boldsymbol{\chi}^{(\tau)} \cdot \nabla w_{1}\right\}}=\delta_{\alpha \tau}, \\
\overline{\left\{\mathbf{V}^{-\top} \boldsymbol{\chi}^{(\tau)}\right\}}=0
\end{gathered}
$$

Here the matrices $\mathbf{S}$ and $\mathbf{T}$ are defined by (4.25)

$$
\begin{array}{r}
\mathbf{S}=\mathbf{V}^{\top} \frac{\partial^{2} H}{\partial \mathbf{y}^{2}}(\boldsymbol{\xi}+\mathbf{u}, \mathbf{v}, \mathbf{w}) \mathbf{V}, \\
\mathbf{T}=\mathbf{W}^{\top} \frac{\partial^{2} H}{\partial \mathbf{z} \partial \mathbf{y}}(\boldsymbol{\xi}+\mathbf{u}, \mathbf{v}, \mathbf{w}) \mathbf{V}+\boldsymbol{\Lambda}^{\top} \frac{\partial^{2} H}{\partial \mathbf{y}^{2}}(\boldsymbol{\xi}+\mathbf{u}, \mathbf{v}, \mathbf{w}) \mathbf{V}
\end{array}
$$

In view of (4.26) and (7.3), they admit the estimates

$$
\left\|\mathbf{S}-\mathbf{S}_{0}\right\|_{\sigma / 2, d-1}+\left\|\mathbf{T}-\mathbf{T}_{0}\right\|_{\sigma / 2, d-1} \leq c|\varepsilon|,
$$

where the constant matrices $\mathbf{S}_{0}, \mathbf{T}_{0}$ are given by

$$
\mathbf{S}_{0}=\frac{\partial^{2} H_{0}}{\partial \mathbf{y}^{2}}(0,0), \quad \mathbf{T}_{0}=\frac{\partial^{2} H_{0}}{\partial \mathbf{z} \partial \mathbf{y}}(0,0)
$$

Proof. Since $\mathfrak{u}=(\boldsymbol{\varphi}, e, m, M)$ is continuously differentiable with respect to $\tau=\alpha, k$ we can differentiate (7.6) with respect to $\tau$ to obtain

$$
\begin{aligned}
D_{\varphi} \Phi_{i}(\mathfrak{f}, \mathfrak{u}) \partial_{\tau} \boldsymbol{\varphi}+D_{e} \Phi_{i}(\mathfrak{f}, \mathfrak{u}) \partial_{\tau} e+ \\
\quad D_{m} \Phi_{i}(\mathfrak{f}, \mathfrak{u}) \partial_{\tau} m+D_{M} \Phi(\mathfrak{f}, \mathfrak{u})\left(\partial_{\tau} M\right)+\partial_{\tau} \Phi_{i}=0 .
\end{aligned}
$$

$i=1, \ldots, 5$. Introduce the functions

$$
\begin{gathered}
\chi^{(\tau)}=\mathbf{V}^{\top} \partial_{\tau} \mathbf{u} \\
\boldsymbol{\lambda}^{(\tau)}=\mathbf{W}^{-1} \partial_{\tau} \mathbf{w}-\chi_{i}^{(\tau)} \mathbf{W}^{-1} \frac{\partial}{\partial \xi_{i}} \mathbf{W} \\
\boldsymbol{\Gamma}^{(\tau)}=\mathbf{W}^{-1} \partial_{\tau} \mathbf{W}-\chi_{i}^{(\tau)} \mathbf{W}^{-1} \frac{\partial}{\partial \xi_{i}} \mathbf{W} \\
\boldsymbol{\mu}^{(\tau)}=\mathbf{V}^{-1}\left(\partial_{\tau} \mathbf{v}+\chi_{i}^{(\tau)} \frac{\partial}{\partial \xi_{i}} \mathbf{v}-\boldsymbol{\Lambda} \boldsymbol{\lambda}^{(\tau)}\right) \\
\nabla \psi_{0}^{(\tau)}=\boldsymbol{\mu}^{(\tau)}-\boldsymbol{\beta}^{(\tau)}, \quad \boldsymbol{\beta}^{(\tau)}=\frac{1}{(2 \pi)^{n-1}} \int_{\mathbb{T}^{n-1}} \boldsymbol{\mu}^{(\tau)} d \boldsymbol{\xi}
\end{gathered}
$$


Since $\mathbf{u}, \mathbf{v}, \mathbf{w}$, and $\mathbf{W}$ form a canonical mapping in the group $\mathcal{G}$, the vector functions $\partial_{\tau} \mathbf{u}, \partial_{\tau} \mathbf{v}, \partial_{\tau} \mathbf{w}$, and $\partial_{\tau} \mathbf{W}$ determine the element of the tangent space of $\mathcal{G}$. Hence we can apply Corollary 4.8 of the structural theorem 4.7 to obtain

$$
\begin{gathered}
D_{\varphi} \Phi_{1}\left[\partial_{\tau} \boldsymbol{\varphi}\right]+D_{e} \Phi_{1}\left[\partial_{\tau} e\right]+D_{m} \Phi_{1}\left[\partial_{\tau} m\right]+D_{M} \Phi_{1}\left[\partial_{\tau} M\right]= \\
\boldsymbol{\partial} \psi_{0}^{(\tau)}+\boldsymbol{\omega}^{\top} \cdot \boldsymbol{\beta}^{\tau}+\partial_{\tau} e+\partial_{\tau} m w_{1}+\partial_{\tau} M \frac{1}{2} w_{1}^{2}, \\
D_{\varphi} \Phi_{2}\left[\partial_{\tau} \boldsymbol{\varphi}\right]+D_{e} \Phi_{2}\left[\partial_{\tau} e\right]+D_{m} \Phi_{2}\left[\partial_{\tau} m\right]+D_{M} \Phi_{b}\left[\partial_{\tau} M\right] \equiv \\
\mathbf{J} \boldsymbol{\partial} \boldsymbol{\lambda}^{(\tau)}+\boldsymbol{\Omega} \boldsymbol{\lambda}^{(\tau)}+\mathbf{T} \boldsymbol{\mu}^{(\tau)}+\partial_{\tau} m \mathbf{W}^{\top} \mathbf{e}_{1}+\partial_{\tau} M w_{1} \mathbf{W}^{\top} \mathbf{e}_{1}, \\
\boldsymbol{\mu}^{(\tau)}=\nabla \psi_{0}^{(\tau)}+\boldsymbol{\beta}^{(\tau)} \\
D_{\varphi} \Phi_{3}\left[\partial_{\tau} \boldsymbol{\varphi}\right]+D_{e} \Phi_{3}\left[\partial_{\tau} e\right]+D_{m} \Phi_{3}\left[\partial_{\tau} m\right]+D_{M} \Phi_{M}\left[\partial_{\tau} M\right] \equiv \\
-\boldsymbol{\partial} \boldsymbol{\chi}^{(\tau)}+\mathbf{S} \boldsymbol{\mu}^{(\tau)}+\mathbf{T}^{\top} \boldsymbol{\lambda}^{(\tau)}, \\
D_{\varphi} \Phi_{5}\left[\partial_{\tau} \boldsymbol{\varphi}\right]+D_{e} \Phi_{5}\left[\partial_{\tau} e\right]+D_{m} \Phi_{3}\left[\partial_{\tau} m\right]+D_{M} \Phi_{M}\left[\partial_{\tau} M\right] \equiv \\
\frac{\boldsymbol{\lambda}^{(\tau)} \cdot \mathbf{W}^{\top} \mathbf{e}_{1}}{\boldsymbol{\chi}^{(\tau)} \cdot \nabla w_{1}}
\end{gathered}
$$

Since $D_{\tau} \Phi_{1}=0$ we have from equality (7.17)

$$
\boldsymbol{\partial} \psi_{0}^{(\tau)}+\boldsymbol{\omega}^{\top} \cdot \boldsymbol{\beta}^{(\tau)}+\partial_{\tau} e+\partial_{\tau} m w_{1}+\partial_{\tau} M \frac{1}{2} w_{1}^{2}=0
$$

Next we have

$$
\nabla\left(\boldsymbol{\partial} \psi_{0}^{(\tau)}+\boldsymbol{\omega}^{\top} \cdot \boldsymbol{\beta}^{(\tau)}\right)=\boldsymbol{\partial} \boldsymbol{\mu}^{(\tau)} .
$$

On the other hand, relations $p^{(\tau)}=\partial_{\tau} m+\alpha \partial_{\tau} M$ and $w_{1}=\alpha+w_{1}^{*}$ yield

$$
\nabla\left(\partial_{\tau} m w_{1}+\partial_{\tau} M \frac{1}{2} w_{1}^{2}\right)=p^{(\tau)} \nabla w_{1}^{*}+\partial_{\tau} M \frac{1}{2} \nabla\left(w_{1}^{*}\right)^{2}
$$

Taking the gradient from both the sides of (7.20) and using equalities (7.21)(7.22) we arrive at (7.13a). Since $\partial_{\tau} \Phi_{2}=0$ it follows from (17.17) and (7.19b) that

$$
\mathbf{J} \boldsymbol{\partial} \boldsymbol{\lambda}^{(\tau)}+\boldsymbol{\Omega} \boldsymbol{\lambda}^{(\tau)}+\mathbf{T} \boldsymbol{\mu}^{(\tau)}+\partial_{\tau} m \mathbf{W}^{\top} \mathbf{e}_{1}+\partial_{\tau} M w_{1} \mathbf{W}^{\top} \mathbf{e}_{1}=0
$$

It is easily seen that

$$
\partial_{\tau} m \mathbf{W}^{\top} \mathbf{e}_{1}+\partial_{\tau} M w_{1} \mathbf{W}^{\top} \mathbf{e}_{1}=p^{(\tau)} \mathbf{W}^{\top} \mathbf{e}_{1}+w_{1}^{*} \mathbf{W}^{\top} \mathbf{e}_{1} .
$$


Substituting this relation into (7.23) we arrive at (7.13b). Next (7.17) and (7.19c) yield (7.13c) since $\partial_{\tau} \Phi_{3}=0$. Next, we have $\partial_{\tau} \Phi_{5}=-\delta_{\tau \alpha}$. Substituting this equality and relation (7.19d) into (7.17) we arrive at (7.13d). It remains to note that equation (7.13e) obviously follows from the equality $\overline{\partial_{\tau} \mathbf{u}}=0$ and relation (7.18a).

Corollary 7.3. Under the assumptions of Theorem 7.1, we have

$$
\begin{aligned}
\left(\boldsymbol{\mu}^{(\alpha)}, \boldsymbol{\lambda}^{(\alpha)}, \boldsymbol{\chi}^{(\alpha)}, p^{(\alpha)}\right)= & \left(\boldsymbol{\mu}^{(1)}, \boldsymbol{\lambda}^{(1)}, \boldsymbol{\chi}^{(1)}, p^{(1)}\right)+\partial_{\alpha} M\left(\boldsymbol{\mu}^{(2)}, \boldsymbol{\lambda}^{(2)}, \boldsymbol{\chi}^{(2)}, p^{(2)}\right) \\
& \left(\boldsymbol{\mu}^{(k)}, \boldsymbol{\lambda}^{(k)}, \boldsymbol{\chi}^{(k)}, p^{(k)}\right)=\partial_{k} M\left(\boldsymbol{\mu}^{(2)}, \boldsymbol{\lambda}^{(2)}, \boldsymbol{\chi}^{(2)}, p^{(2)}\right),
\end{aligned}
$$

Where $\left(\boldsymbol{\mu}^{(i)}, \boldsymbol{\lambda}^{(i)}, \boldsymbol{\chi}^{(i)}, q^{(i)}\right), i=1,2$ are solutions to the equations

$$
\begin{gathered}
\boldsymbol{\partial} \boldsymbol{\mu}^{(1)}=-p^{(1)} \nabla w_{1}, \\
\mathbf{J} \boldsymbol{\partial} \boldsymbol{\lambda}^{(1)}+\boldsymbol{\Omega} \boldsymbol{\lambda}^{(1)}+\mathbf{T} \boldsymbol{\mu}^{(1)}+p^{(1)} \mathbf{W}^{\top} \boldsymbol{e}_{1}=0, \\
-\boldsymbol{\partial} \boldsymbol{\lambda}^{(1)}+\mathbf{S} \boldsymbol{\mu}^{(1)}+\mathbf{T}^{\top} \boldsymbol{\lambda}^{(1)}=0 \\
\overline{\left\{W \boldsymbol{\lambda}^{(1)} \cdot \mathbf{e}_{1}\right\}}+\overline{\left\{\boldsymbol{\chi}^{(1)} \cdot \nabla w_{1}\right\}}=1, \\
\frac{\left\{\mathbf{V}^{-\top} \boldsymbol{\chi}^{(1)}\right\}}{2}=0,
\end{gathered}
$$

and

$$
\begin{aligned}
& \boldsymbol{\partial} \boldsymbol{\mu}^{(2)}=-p^{(2)} \nabla w_{1}-\frac{1}{2} \nabla\left(w_{1}^{*}\right)^{2}, \\
& \mathbf{J} \boldsymbol{\partial} \boldsymbol{\lambda}^{(2)}+\boldsymbol{\Omega} \boldsymbol{\lambda}^{(2)}+\mathbf{T} \boldsymbol{\mu}^{(2)}+p^{(2)} \mathbf{W}^{\top} \boldsymbol{e}_{1}=-w_{1}^{*} \mathbf{W}^{\top} \boldsymbol{e}_{1}, \\
& -\boldsymbol{\partial} \boldsymbol{\lambda}^{(2)}+\mathbf{S} \boldsymbol{\mu}^{(2)}+\mathbf{T}^{\top} \boldsymbol{\lambda}^{(2)}=0 \\
& \overline{\left\{W \boldsymbol{\lambda}^{(2)} \cdot \mathbf{e}_{1}\right\}}+\overline{\left\{\boldsymbol{\chi}^{(2)} \cdot \nabla w_{1}\right\}}=0, \\
& \overline{\left\{\mathbf{V}^{-\top} \boldsymbol{\chi}^{(2)}\right\}}=0
\end{aligned}
$$

In the next section we investigate problems (7.25) and (7.26) in many details. 


\section{Linear problem}

In this section we prove the solvability of problems (7.25) and (7.26) and establish estimates for their solutions in the spaces of analytic functions and the Sobolev spaces. First we recall the basic facts from the theory of Sobolev spaces on torus.

\subsection{Preliminaries. Sobolev spaces}

For every $s \in \mathbb{R}$, we denote by $H_{s}$ the Hilbert space which consists of all distributions $u$ on the tori $\mathbb{T}^{n-1}$ such that

$$
|u|_{s}=\left(\sum_{m \in \mathbb{Z}^{n-1}}\left(1+|m|^{2}\right)^{s}|\hat{u}(m)|^{2}\right)^{1 / 2},
$$

where $\hat{u}$ is the Fourier transform of $u$. If $u$ is an integrable function, then

$$
\hat{u}(m)=(2 \pi)^{-(n-1) / 2} \int_{\mathbb{T}^{n-1}} e^{-i m \cdot \boldsymbol{\xi}} u(\boldsymbol{\xi}) d \boldsymbol{\xi} .
$$

It is clear that $H_{0}=L^{2}\left(\mathbb{T}^{n-1}\right)$ and

$$
|u|_{s}=\left\|(-\Delta+1)^{s / 2} u\right\|_{L^{2}\left(\mathbb{T}^{n-1}\right)} .
$$

For every $s^{\prime}<s^{\prime \prime}$ the embedding $H_{s^{\prime \prime}} \hookrightarrow H_{s^{\prime}}$ is compact. For every integer $l \geq 0$ the embedding $H_{n-1+l} \hookrightarrow C^{l}\left(\mathbb{T}^{n-1}\right)$ is compact. Next, we have

$$
|u v|_{s} \leq c(s)|u|_{s}\|v\|_{s} \text { for } s \geq n-1
$$

For all $-\infty<r \leq s<\infty$ we have the interpolation inequality

$$
\|u\|_{(s+r) / 2} \leq c(s, r)\|u\|_{s}^{1 / 2}\|u\|_{r}^{1 / 2} .
$$

Recall the estimate $|\hat{u}(m)| \leq\|u\|_{\sigma, 0} \exp (-\sigma|m|)$. It follows that

$$
|u|_{s} \leq c(s, \sigma)\|u\|_{\sigma, 0} \text { for all } s \in \mathbb{R} \text { and } \sigma>0
$$




\subsection{Basic linear problem.}

In this section we prove the solvability of linear problems (7.25) and (17.26). We investigate the qualitative properties of their solutions. First we consider the general boundary value problem which includes problems (7.25) and (7.26) as particular cases.

$$
\begin{gathered}
\boldsymbol{\partial} \boldsymbol{\mu}=-p \nabla w_{1}-\nabla g_{1}, \\
\mathbf{J} \boldsymbol{\partial}+\boldsymbol{\Omega} \boldsymbol{\lambda}+\mathbf{T} \boldsymbol{\mu}+p \mathbf{W}^{\top} \mathbf{e}_{1}=\mathbf{g}_{2} \\
-\boldsymbol{\partial} \boldsymbol{\lambda}+\mathbf{S} \boldsymbol{\mu}+\mathbf{T}^{\top} \boldsymbol{\lambda}=0 \\
\overline{\{W \boldsymbol{\lambda}\} \cdot \mathbf{e}_{1}}+\overline{\left\{\boldsymbol{\chi} \cdot \nabla w_{1}\right\}}=\gamma, \\
\overline{\left\{\mathbf{V}^{-\top} \boldsymbol{\chi}\right\}}=0
\end{gathered}
$$

Assume that $w_{1}$ and $\mathbf{W}$ meet all requirements of Theorem 7.1 , i.e.

$$
\left\|\mathbf{V}^{-1}-\mathbf{I}\right\|_{\sigma / 2,0}+\left\|\left(w_{1}-\alpha\right)\right\|_{\sigma / 2, d}+\|\mathbf{W}-\mathbf{I}\|_{\sigma / 2, d} \leq c|\varepsilon|
$$

We also assume that the matrix -valued functions $\mathbf{S}$ and $\mathbf{T}$ meet all requirements of Lemma 7.2, i.e.

$$
\left\|\mathbf{S}-\mathbf{S}_{0}\right\|_{\sigma / 2, d-1}+\left\|\mathbf{T}-\mathbf{T}_{0}\right\|_{\sigma / 2, d-1} \leq c|\varepsilon|,
$$

where the constant matrices $\mathbf{S}_{0}, \mathbf{T}_{0}$ are given by

$$
\mathbf{S}_{0}=\frac{\partial^{2} H_{0}}{\partial \mathbf{y}^{2}}(0,0), \quad \mathbf{T}_{0}=\frac{\partial^{2} H_{0}}{\partial \mathbf{z} \partial \mathbf{y}}(0,0)
$$

Recall that $d \geq 2$ is a fixed number. The following theorem is the main result of this section.

Theorem 8.1. Let $s \in \mathbb{R}^{1}$ be an arbitrary number and $\sigma \in(1 / 2,1]$. Then there is $\varepsilon_{0}>0$ independent on $\alpha$ and $k$ with the following properties. For every $|\varepsilon| \leq \varepsilon_{0}, g_{1}, \mathbf{g}_{2} \in \mathcal{A}_{\sigma / 2,0}$ and $\gamma \in \mathbb{R}^{1}$, problem (8.5) has a unique solution $(\boldsymbol{\mu}, \boldsymbol{\lambda}, \boldsymbol{\chi}) \in \mathcal{A}_{\sigma / 4,0}, p \in \mathbb{R}^{1}$. This solution admits the estimates

$$
\begin{gathered}
\|\boldsymbol{\mu}\|_{\sigma / 4,0}+\|\boldsymbol{\lambda}\|_{\sigma / 4,0}+\|\boldsymbol{\chi}\|_{\sigma / 4,0}+|p| \leq c\left(\left\|g_{1}\right\|_{\sigma / 2,0}+\left\|\boldsymbol{g}_{2}\right\|_{\sigma / 2,0}+|\gamma|\right) \\
|\boldsymbol{\mu}|_{s}+|\boldsymbol{\lambda}|_{s}+|\boldsymbol{\chi}|_{s}+|p| \leq c\left(\left|g_{1}\right|_{s+4 n+6}+\left|\boldsymbol{g}_{2}\right|_{s+4 n+6}+|\gamma|\right) \\
|\overline{\boldsymbol{\mu}}| \leq c\left|\overline{\mathbf{g}}_{2}\right|+\varepsilon_{0} c\left(\left|g_{1}\right|_{s+4 n+6}+\left|\boldsymbol{g}_{2}\right|_{s+4 n+6}+|\gamma|\right)
\end{gathered}
$$


Proof. The proof is in Appendix E.2.

We employ Theorem 8.1 in order to investigate the structure of solutions to problems (7.25) and (7.26) and to establish robust estimates of these solutions. We start with detailed analysis of problem (7.26). The corresponding results is given by the following theorem which is the second main statement of this section.

Theorem 8.2. Under the assumptions of of Theorem 8.1, there exists $\varepsilon_{0}>0$ with the following property. For every $|\varepsilon| \leq \varepsilon_{0}$, problem (17.26) has a unique analytic solution $\left(\boldsymbol{\mu}^{(2)}, \boldsymbol{\lambda}^{(2)}, \boldsymbol{\chi}^{(2)}\right) \in \mathcal{A}_{\sigma / 4,0}, p^{(2)} \in \mathbb{C}$. This solution admits the estimate

$$
\begin{gathered}
\left\|\boldsymbol{\mu}^{(2)}\right\|_{\sigma / 4,0}+\left\|\boldsymbol{\lambda}^{(2)}\right\|_{\sigma / 4,0}+\left\|\chi^{(2)}\right\|_{\sigma / 4,0}+\left|p^{(2)}\right| \leq c\left\|w_{1}^{*}\right\|_{\sigma / 2,0} \leq c|\varepsilon|, \\
\left|\boldsymbol{\mu}^{(2)}\right|_{0} \leq c \varepsilon_{0}\left|w_{1}^{*}\right|_{-1}, \quad\left|\lambda_{2}^{(2)}\right|_{0}+|k|\left|\lambda_{1}^{(2)}\right|_{0} \geq c^{-1}\left|w_{1}^{*}\right|_{-1} .
\end{gathered}
$$

Here the strictly positive constant $c$ is independent of $\alpha, k$, and $\varepsilon$.

Proof. Notice that problem (7.26) is a particular case of problem (8.5) with the right hand sides

$$
g_{1} *=\left(w_{1}^{*}\right)^{2}, \quad \mathbf{g}_{2}=-w_{1}^{*} \mathbf{W e}_{1}, \quad \gamma=0 .
$$

It follows from inequalities (8.6) and the identity $w_{1}^{*}=w_{1}-\alpha$ that

$$
\left\|g_{1}^{*}\right\|_{\sigma / 2,0} \leq c\left\|w_{1}^{*}\right\|_{\sigma / 2,0}^{2}, \quad\left\|\mathbf{g}_{2}\right\|_{\sigma / 2,0} \leq c\left\|w_{1}^{*}\right\|_{\sigma / 2,0} .
$$

Applying Theorem 8.1 we conclude that problem (7.26) has a unique solution, satisfying inequality (8.12). It remains to prove estimates (8.13) Choose an arbitrary $t>n$. Since the space $H_{t+1}$ is a Banach algebra, we have

$$
\left|\nabla\left(w_{1}^{*}\right)^{2}\right|_{t} \leq\left|\left(w_{1}^{*}\right)^{2}\right|_{t+1} \leq c\left|w_{1}^{*}\right|_{t+1}^{2}
$$

On the other hand, the interpolation inequality implies

$$
\left|w_{1}^{*}\right|_{t+1} \leq c\left|w_{1}^{*}\right|_{-1}^{1 / 2}\left|w_{1}^{*}\right|_{2 t+3}^{1 / 2} \leq c\left|w_{1}^{*}\right|_{-1}^{1 / 2}\left\|w_{1}^{*}\right\|_{\sigma / 2,0}^{1 / 2} \leq c \varepsilon_{0}^{1 / 2}\left|w_{1}^{*}\right|_{-1}^{1 / 2} .
$$

We thus get

$$
\left|\nabla\left(w_{1}^{*}\right)^{2}\right|_{t} \leq c|\varepsilon|\left|w_{1}^{*}\right|_{-1} .
$$


Obviously, this inequality holds true for all $t \in \mathbb{R}$. Next we have

$$
\left|w_{1}^{*} \mathbf{W} \mathbf{e}_{1}\right|_{t} \leq c|| \mathbf{W} \|_{\sigma / 2,0}\left|w_{1}^{*}\right|_{t} \leq c\left|w_{1}^{*}\right|_{t}
$$

Now set $s=-1-4 n-6$ and $t=-1$. Apply Theorem 8.1 to problem (7.26). It follows from the estimate (8.10) in this theorem and relations (8.13)-(8.16) that

$$
\left|\boldsymbol{\mu}^{(2)}\right|_{-4 n-7}+\left|\boldsymbol{\lambda}^{(2)}\right|_{-4 n-7}+\left|\boldsymbol{\chi}^{(2)}\right|_{-4 n-7}+\left|p^{(2)}\right| \leq c\left|\mathbf{w}_{1}^{*}\right|_{-1} .
$$

Since $s+4 n+6=-1$, estimate (8.11) implies

$$
\left|\overline{\boldsymbol{\mu}^{(2)}}\right| \leq\left|\overline{\mathbf{g}_{2}}\right|+c \varepsilon_{0}\left|\mathbf{w}_{1}^{*}\right|_{-1} .
$$

We have

$$
\left|\overline{\mathbf{g}_{2}}\right|=\left|\overline{w_{1}^{*} \mathbf{W} \mathbf{e}_{1}}\right|=\left|\overline{w_{1}^{*}(\mathbf{W}-\mathbf{I}) \mathbf{e}_{1}}\right| \leq\left|w_{1}^{*}\right|_{-1}|\mathbf{W}-\mathbf{I}|_{1} \leq c \varepsilon_{0}\left|w_{1}^{*}\right|_{-1} .
$$

Combining the obtained results we arrive at the estimate

$$
\left|\overline{\boldsymbol{\mu}^{(2)}}\right| \leq c \varepsilon_{0}\left|\mathbf{w}_{1}^{*}\right|_{-1}
$$

Let us estimate $\boldsymbol{\mu}^{(2)^{*}}$. The first equation (7.26a) in system (17.26) reads

$$
\boldsymbol{\partial} \boldsymbol{\mu}^{(2)^{*}}=-p^{(2)} \nabla w_{1}-\frac{1}{2} \nabla\left(w_{1}^{*}\right)^{2}
$$

Applying Lemma E.2 to this equation we obtain

$$
\left|\boldsymbol{\mu}^{(2)^{*}}\right|_{0} \leq c\left|p^{(2)}\right|\left|w_{1}^{*}\right|_{4 n+7}+c\left|\nabla\left(w_{1}^{*}\right)^{2}\right|_{4 n+6}
$$

It follows from inequality (8.15) with $t=4 n+6$ that

$$
\left|w_{1}^{*}\right|_{4 n+7} \leq c\left\|w_{1}^{*}\right\|_{\sigma / 2,0} \leq c \varepsilon_{0}, \quad\left|\nabla\left(w_{1}^{*}\right)^{2}\right|_{4 n+6} \leq c \varepsilon_{0}\left|w_{1}^{*}\right|_{-1} .
$$

It follows that

$$
\left|\boldsymbol{\mu}^{(2)^{*}}\right|_{0} \leq c \varepsilon_{0}\left|w_{1}^{*}\right|_{-1} .
$$

Combining this result with (8.18) we obtain the first estimate in (8.13). Let us prove the second. Notice that $\left|\boldsymbol{\partial} \lambda_{2}^{(2)}\right|_{-1} \leq c\left|\boldsymbol{\lambda}_{2}^{(2)}\right|_{0}$. It follows that

$$
\begin{gathered}
\left|\lambda_{2}^{(2)}\right|_{0}+|k|\left|\lambda_{1}^{(2)}\right|_{0} \geq c^{-1}\left(\left|\boldsymbol{\partial} \lambda_{2}^{(2)}\right|_{-1}+|k|\left|\lambda_{1}^{(2)}\right|_{-1}\right) \geq \\
c^{-1}\left(\left|\boldsymbol{\partial} \lambda_{2}^{(2)}-k \lambda_{1}^{(2)}\right|_{-1}\right) \geq c^{-1}\left(\left|\boldsymbol{\partial} \lambda_{2}^{(2)^{*}}-k \lambda_{1}^{(2)^{*}}\right|_{-1}\right) .
\end{gathered}
$$


On the other hand, the second equation (7.26b) in system (7.26) implies

$$
\boldsymbol{\partial} \lambda_{2}^{(2)^{*}}-k \lambda_{1}^{(2)^{*}}=-w_{1}^{*}-\left(\mathbf{T} \boldsymbol{\mu}^{(2)}\right)^{*}-p^{(2)}\left(\mathbf{W}^{\top}-\mathbf{I}\right)^{*} \mathbf{e}_{1}-\left(w_{1}^{*}\left(\mathbf{W}^{\top}-\mathbf{I}\right) \mathbf{e}_{1}\right)^{*}
$$

We have

$$
\begin{gathered}
\left|\left(\mathbf{W}^{\top}-\mathbf{I}\right)^{*}\right|_{-1} \leq\left\|\left(\mathbf{W}^{\top}-\mathbf{I}\right)^{*}\right\|_{\sigma / 2,0} \leq c \varepsilon_{0} \\
\left|w_{1}^{*}\left(\mathbf{W}^{\top}-\mathbf{I}\right) \mathbf{e}_{1}\right|_{-1} \leq c\left|w_{1}^{*}\right|_{-1}\left\|\left(\mathbf{W}^{\top}-\mathbf{I}\right)^{*}\right\|_{\sigma / 2,0} \leq c \varepsilon_{0}\left|w_{1}^{*}\right|_{-1} .
\end{gathered}
$$

Next, the first estimate in (8.13) implies

$$
\left|\mathbf{T} \boldsymbol{\mu}^{(2)}\right|_{0} \leq c \varepsilon_{0}\left|w_{1}^{*}\right|_{-1} .
$$

Combining obtained inequalities we arrive at the estimate

$\left|w_{1}^{*}+\left(\mathbf{T} \boldsymbol{\mu}^{(2)}\right)^{*}+p^{(2)}\left(\mathbf{W}^{\top}-\mathbf{I}\right)^{*} \mathbf{e}_{1}+\left(w_{1}^{*}\left(\mathbf{W}^{\top}-\mathbf{I}\right) \mathbf{e}_{1}\right)^{*}\right|_{-1} \geq\left|w_{1}^{*}\right|_{-1}-c \varepsilon_{0}\left|w_{1}^{*}\right|_{-1}$,

which yields

$$
\left|\boldsymbol{\partial} \lambda_{2}^{(2)^{*}}-k \lambda_{1}^{(2)^{*}}\right|_{-1} \geq\left|w_{1}^{*}\right|_{-1}-c \varepsilon_{0}\left|w_{1}^{*}\right|_{-1} .
$$

This estimate along with (8.19) implies the estimate

$$
\left|\lambda_{2}^{(2)}\right|_{0}+|k|\left|\lambda_{1}^{(2)}\right|_{0} \geq c^{-1}\left(\left|w_{1}^{*}\right|_{-1}-c \varepsilon_{0}\left|w_{1}^{*}\right|_{-1}\right) .
$$

Choosing $\varepsilon_{0}$ sufficiently small we obtain the second estimate in (8.13) and the theorem follows.

Now turn to problem (7.25). Our goal is to prove the existence and uniqueness of solutions to this problem and to obtain the asymptotic expansion of its solutions near $\varepsilon=0$. In order to formulate the results we introduce the auxiliary denotations. Recall denotations (7.14)-(7.16) for the matrix $\mathbf{T}$. Denote by $\mathbf{t}_{i}^{\top}, i=1,2$, the rows of $\mathbf{T}$. They can be regarded al analytic periodic functions $\mathbf{t}_{i}: \mathbb{T}^{n-1} \rightarrow \mathbb{C}^{n-1}$. Notice that for $\varepsilon=0$, we have $\mathbf{t}_{1}=0$ and $\mathbf{t}_{2}=\mathbf{t}_{0}$, where $\mathbf{t}_{0}$ is the only nonzero row of the matrix $\mathbf{T}_{0}$. It follows from this and estimate (7.15) that

$$
\left\|\mathbf{t}_{1}\right\|_{\sigma / 2, d-1}+\left\|\mathbf{t}_{2}-\mathbf{t}_{0}\right\|_{\sigma / 2, d-1} \leq c|\varepsilon| .
$$

Recall the denotation

$$
\overline{\mathbf{t}_{1}}=\frac{1}{(2 \pi)^{n-1}} \int_{\mathbb{T}^{n-1}} \mathbf{t}_{1} d \boldsymbol{\xi}
$$


Introduce the constant vector

$$
\boldsymbol{\mu}_{0}=\mathbf{K}_{0}^{-1} \overline{\mathbf{t}_{1}} .
$$

Our considerations are based on the following algebraic lemma.

Lemma 8.3. Let $k=0$. There is $\varepsilon_{0}>0$ with the following properties. For every $|\varepsilon| \leq \varepsilon_{0}$, the system of linear equations

$$
\begin{aligned}
\boldsymbol{\Omega} \boldsymbol{\lambda}_{1}+\overline{\mathbf{T}} \boldsymbol{\mu}_{1}+p_{1} \mathbf{e}_{1} & =0 \\
\overline{\mathbf{T}}^{\top} \boldsymbol{\lambda}_{1}+\overline{\mathbf{S}} \boldsymbol{\mu}_{1} & =0 \\
\boldsymbol{\lambda}_{1} \mathbf{e}_{1} & =1 .
\end{aligned}
$$

has a unique solution $\boldsymbol{\lambda}_{1} \in \mathbb{C}^{2}, \boldsymbol{\mu}_{1} \in \mathbb{C}^{n-1}, p_{1} \in \mathbb{C}$ such that

$$
\begin{gathered}
\boldsymbol{\mu}_{1}=\boldsymbol{\mu}_{0}+\boldsymbol{\mu}_{2}, \quad \boldsymbol{\lambda}_{1}=\mathbf{e}_{1}-\left(\mathbf{t}_{0} \cdot \boldsymbol{\mu}_{0}\right) \mathbf{e}_{2}+\boldsymbol{\lambda}_{2} \\
\left|\boldsymbol{\mu}_{2}\right| \leq c|\varepsilon|\left|\boldsymbol{\mu}_{0}\right|, \quad\left|p_{1}\right| \leq c|\varepsilon|\left|\boldsymbol{\mu}_{0}\right|, \quad\left|\boldsymbol{\lambda}_{2}\right| \leq c|\varepsilon|\left|\boldsymbol{\mu}_{0}\right| .
\end{gathered}
$$

Proof. Rewrite the first two equations in the form

$$
\begin{array}{r}
\boldsymbol{\Omega} \boldsymbol{\lambda}_{1}+\mathbf{T}_{0} \boldsymbol{\mu}_{1}+p_{1} \mathbf{e}_{1}=\left(\mathbf{T}_{0}-\overline{\mathbf{T}}\right) \boldsymbol{\mu}_{1}, \\
\mathbf{T}_{0}^{\top} \boldsymbol{\lambda}_{1}+\mathbf{S}_{0} \boldsymbol{\mu}_{1}=\left(\mathbf{T}_{0}^{\top}-\overline{\mathbf{T}}^{\top}\right) \boldsymbol{\lambda}_{1}+\left(\mathbf{S}_{0}-\overline{\mathbf{S}}\right) \boldsymbol{\mu}_{1} .
\end{array}
$$

Notice that $\boldsymbol{\lambda}_{1}=\lambda_{1,2} \mathbf{e}_{2}+\mathbf{e}_{1}$. Thus we get the linear system of the equations for $\lambda_{1,2}$ and $\boldsymbol{\mu}_{1}$

$$
\begin{gathered}
\lambda_{1,2}+\mathbf{t}_{0} \cdot \boldsymbol{\mu}_{1}=\left\{\left(\mathbf{T}_{0}-\overline{\mathbf{T}}\right) \boldsymbol{\mu}_{1}\right\} \cdot \mathbf{e}_{2} \\
\mathbf{S}_{0} \boldsymbol{\mu}_{1}+\lambda_{1,2} \mathbf{t}_{0}=\left(\mathbf{T}_{0}^{\top}-\overline{\mathbf{T}}^{\top}\right) \mathbf{e}_{1}+ \\
\lambda_{1,2}\left(\mathbf{T}_{0}^{\top}-\overline{\mathbf{T}}^{\top}\right) \mathbf{e}_{2}+\left(\mathbf{S}_{0}-\overline{\mathbf{S}}\right) \boldsymbol{\mu}_{1} .
\end{gathered}
$$

Express $\lambda_{1,2}$ in terms of $\boldsymbol{\mu}_{1}$ using the first equation in (8.25). Substituting the result into the second equation in (8.25) we obtain the following equation for $\boldsymbol{\mu}_{1}$

$$
\begin{array}{r}
\mathbf{K}_{0} \boldsymbol{\mu}_{1}=\left(\mathbf{S}_{0}-\overline{\mathbf{S}}\right) \boldsymbol{\mu}_{1}+\left\{\left(\mathbf{T}_{0}-\overline{\mathbf{T}}\right) \boldsymbol{\mu}_{1}\right\}_{2}\left(\left(\mathbf{T}_{0}^{\top}-\overline{\mathbf{T}}^{\top}\right) \boldsymbol{e}_{2}-\mathbf{t}_{0}\right) \\
-\left(\boldsymbol{\mu}_{1} \cdot \mathbf{t}_{0}\right)\left(\mathbf{T}_{0}^{\top}-\overline{\mathbf{T}}^{\top}\right) \mathbf{e}_{2}+\left(\mathbf{T}_{0}^{\top}-\overline{\mathbf{T}}^{\top}\right) \boldsymbol{e}_{1},
\end{array}
$$


where the matrix $\mathbf{K}_{0}=\mathbf{S}_{0}-\mathbf{t}_{0} \otimes \mathbf{t}_{0}$ has a bounded inverse. Using the identities $\left(\mathbf{T}_{0}^{\top}-\overline{\mathbf{T}}^{\top}\right) \boldsymbol{e}_{2}-\mathbf{t}_{0}=-\overline{\mathbf{t}}_{2}$ and $\left(\mathbf{T}_{0}^{\top}-\overline{\mathbf{T}}^{\top}\right) \boldsymbol{e}_{1}=\overline{\mathbf{t}}_{1}$, we can rewrite this equation in the equivalent form

$$
\begin{array}{r}
\mathbf{K}_{0} \boldsymbol{\mu}_{1}=\left(\mathbf{S}_{0}-\overline{\mathbf{S}}\right) \boldsymbol{\mu}_{1}-\left\{\left(\mathbf{T}_{0}-\overline{\mathbf{T}}\right) \boldsymbol{\mu}_{1}\right\}_{2} \overline{\mathbf{t}}_{2} \\
-\left(\boldsymbol{\mu}_{1} \cdot \mathbf{t}_{0}\right)\left(\mathbf{T}_{0}^{\top}-\overline{\mathbf{T}}^{\top}\right) \mathbf{e}_{2}-\overline{\mathbf{t}}_{1}
\end{array}
$$

Thus we get the following equation for $\boldsymbol{\mu}_{0}$

$$
\boldsymbol{\mu}_{1}-\mathrm{A} \boldsymbol{\mu}_{1}=-\boldsymbol{\mu}_{0}
$$

where the linear mapping $\mathbf{A}: \mathbb{C}^{2} \rightarrow \mathbb{C}^{2}$ is given by

$$
\mathbf{A}: \boldsymbol{\mu}_{1} \mapsto \mathbf{K}_{0}^{-1}\left\{\left(\mathbf{S}_{0}-\overline{\mathbf{S}}\right) \boldsymbol{\mu}_{1}-\left\{\left(\mathbf{T}_{0}-\overline{\mathbf{T}}\right) \boldsymbol{\mu}\right\}_{2} \overline{\mathbf{t}}_{2}-\left(\boldsymbol{\mu}_{1} \cdot \mathbf{t}_{0}\right)\left(\left(\mathbf{T}_{0}^{\top}-\overline{\mathbf{T}}^{\top}\right) \boldsymbol{e}_{2}\right\}\right.
$$

In view of estimates (7.14), the mapping $\mathbf{A}$ admits the estimate

$$
\left|\mathbf{A} \boldsymbol{\mu}_{1}\right| \leq c|\varepsilon|\left|\boldsymbol{\mu}_{1}\right|
$$

Choosing $\varepsilon_{0}$ sufficiently small we obtain that equation (8.27) has a unique solution which admits decomposition estimate (8.23) with the reminder

$$
\boldsymbol{\mu}_{2}=\left(\mathbf{I}-(\mathbf{I}-\mathbf{A})^{-1}\right) \boldsymbol{\mu}_{0}
$$

When equation (8.27) is solved the vector $\boldsymbol{\lambda}_{1}$ and the scalar $p_{1}$ are restored by the relations

$$
\begin{aligned}
& \boldsymbol{\lambda}_{1}=\mathbf{e}_{1}-\left(\mathbf{t}_{0} \cdot \boldsymbol{\mu}_{1}\right) \mathbf{e}_{2} \\
& p_{1}=\left(\mathbf{T}_{0}-\overline{\mathbf{T}}\right) \boldsymbol{\mu}_{1} \cdot \mathbf{e}_{1}
\end{aligned}
$$

which gives decomposition (8.23) with the reminder

$$
\lambda_{2}=-\left(\mathbf{t}_{0} \cdot \boldsymbol{\mu}_{1}\right) \mathbf{e}_{2}
$$

Estimate (8.24) for $\boldsymbol{\lambda}_{2}$ and $p_{1}$ obviously follows from estimate (8.24) for $\boldsymbol{\mu}_{2}$ and the decomposition (8.23) for $\boldsymbol{\mu}_{1}$.

The following theorem, which is the third main result of this section, constitutes the properties of solutions to problem (17.26) 
Theorem 8.4. Under the assumptions of of Theorem 8.1, there exists $\varepsilon_{0}>0$ with the following property. For every $|\varepsilon| \leq \varepsilon_{0}$, problem (7.25) has a unique analytic solution $\left(\boldsymbol{\mu}^{(1)}, \boldsymbol{\lambda}^{(1)}, \boldsymbol{\chi}^{(1)}\right) \in \mathcal{A}_{\sigma / 4,0}, p^{(1)} \in \mathbb{C}$. This solution admits the estimate

$$
\left\|\boldsymbol{\mu}^{(1)}\right\|_{\sigma / 4,0}+\left\|\boldsymbol{\lambda}^{(1)}\right\|_{\sigma / 4,0}+\left\|\chi^{(1)}\right\|_{\sigma / 4,0}+\left|p^{(1)}\right| \leq c .
$$

If $k=0$, then this solution has the decomposition

$$
\boldsymbol{\mu}^{(1)}=-\boldsymbol{\mu}_{0}+\boldsymbol{\mu}_{\varepsilon}, \quad \boldsymbol{\lambda}^{(1)}=\mathcal{C} \mathbf{e}_{1}-\left(\boldsymbol{\mu}_{0} \cdot \mathbf{t}_{0}\right) \mathbf{e}_{2}+\boldsymbol{\lambda}_{\varepsilon} .
$$

Here the the constant $\mathcal{C}$ and functions $\boldsymbol{\mu}_{\varepsilon}, \boldsymbol{\lambda}_{\varepsilon}$ admit the estimates

$$
|\mathcal{C}-1| \leq c|\varepsilon|, \quad\left\|\boldsymbol{\mu}_{\varepsilon}\right\|_{\sigma / 4,0}+\left\|\boldsymbol{\lambda}_{\varepsilon}\right\|_{\sigma / 4,0} \leq c|\varepsilon|\left|\boldsymbol{\mu}_{0}\right|
$$

The constant $c$ is independent of $\alpha, k$, and $\varepsilon$.

Proof. Notice that problem (7.26) is a particular case of problem (8.5) with the right hand sides $g_{1} *=0, \mathbf{g}_{2}=0$, and $\gamma=1$. Hence, the existence of a solution and estimate (8.29) is a straightforward consequence of Theorem 8.1. It remains to prove decompositiion (8.31).

Now our task is to justify decomposition (8.30). Let $\boldsymbol{\mu}_{1}, \boldsymbol{\lambda}_{1}$, and $p_{1}$ be given by Lemma 8.3 , Set

$$
\chi_{1}=\overline{\chi_{1}}+\chi_{1}^{*}
$$

Here $\chi_{1}^{*}$ is a solution solution to the equations

$$
\partial \chi_{1}^{*}=\mathbf{S} \mu_{1}+\mathbf{T}^{\top} \boldsymbol{\lambda}_{1}, \quad \overline{\chi_{1}^{*}}=0 .
$$

By virtue of (8.22), the mean value of the right hand side of equation (8.32) over the torus $\mathbb{T}^{n-1}$ is equal to zero. Hence this problem have an analytic periodic solution. The constant vector $\overline{\chi_{1}}$ is defined by

$$
\overline{\chi_{1}}=-\overline{\left(\mathbf{V}^{-\top}-\mathbf{I}\right) \chi^{*}}
$$

Represent the solution $\left(\boldsymbol{\mu}^{(1)}, \boldsymbol{\lambda}^{(1)}, \boldsymbol{\chi}^{(1)}, p^{(1)}\right)$ to problem (7.25) in the form

$$
\boldsymbol{\mu}^{(1)}=\mathcal{C} \boldsymbol{\mu}_{1}+\boldsymbol{\mu}, \quad \boldsymbol{\lambda}^{(1)}=\mathcal{C} \boldsymbol{\lambda}_{1}+\boldsymbol{\lambda}, \quad \boldsymbol{\chi}^{(1)}=\mathcal{C} \boldsymbol{\chi}_{1}+\boldsymbol{\chi}, \quad p^{(1)}=\mathcal{C} \boldsymbol{p}_{1}+\boldsymbol{p}
$$

where the constant $\mathcal{C}$ will be specified below. The calculations show that

$$
\begin{gathered}
\boldsymbol{\partial} \boldsymbol{\mu}_{1}=0 \\
\mathbf{J} \boldsymbol{\partial} \boldsymbol{\lambda}_{1}+\boldsymbol{\Omega} \boldsymbol{\lambda}_{1}+\mathbf{T} \boldsymbol{\mu}_{1}+p_{1} \mathbf{W}^{\top} \mathbf{e}_{1}=p_{1}\left(\mathbf{W}^{\top}-\mathbf{I}\right) \mathbf{e}_{1} \\
-\boldsymbol{\partial} \boldsymbol{\chi}_{1}+\mathbf{S} \boldsymbol{\mu}_{1}+\mathbf{T}^{\top} \boldsymbol{\lambda}_{1}=0
\end{gathered}
$$


Next, notice that $\mathbf{V}^{-\top}-\mathbf{I}=\mathbf{u}^{\prime}$, which yields

$$
\overline{\mathbf{V}^{-\top}-\mathbf{I}}=\overline{\mathbf{u}^{\prime}}=0, \quad \overline{\left(\mathbf{V}^{-\top}-\mathbf{I}\right) \boldsymbol{\chi}_{1}}=\overline{\left(\mathbf{V}^{-\top}-\mathbf{I}\right) \boldsymbol{\chi}_{1}^{*}}
$$

It follows from this and (8.33) that

$$
\overline{\left\{\mathbf{V}^{-\top} \boldsymbol{\chi}_{1}\right\}}=0
$$

Finally set

$$
\mathcal{C}=\left\{\overline{\left\{\mathbf{W} \boldsymbol{\lambda}_{1}\right\} \cdot \mathbf{e}_{1}}+\overline{\left\{\boldsymbol{\chi}_{1} \cdot \nabla w_{1}\right\}}\right\}^{-1}
$$

Substituting decomposition (8.34) in equations (7.25) and using relations (8.35) we conclude that the quantities $(\boldsymbol{\mu}, \boldsymbol{\lambda}, \boldsymbol{\chi}, p)$ in (8.34) satisfy equations (8.5) with the right hand sides

$$
g_{1}=\mathcal{C} p_{1} w_{1}^{*}, \quad \boldsymbol{g}_{2}=-\mathcal{C} p_{1}\left(\mathbf{W}^{\top}-\mathbf{I}\right) \mathbf{e}_{1}, \quad \gamma=0 .
$$

In order to apply Theorem (8.1) to the obtained problem (8.5), we have to estimate the the constant $\mathcal{C}$ and functions $g_{1}, \mathbf{g}_{2}$. In order to estimate $\mathcal{C}$, we substitute decomposition (8.23) into the right hand side of relation (8.35e). We get

$$
\left\{\mathbf{W} \boldsymbol{\lambda}_{1}\right\} \cdot \mathbf{e}_{1}=1+\boldsymbol{\lambda}_{2}+\left(\left\{(\mathbf{W}-\mathbf{I}) \boldsymbol{\lambda}_{1}\right\} \cdot \mathbf{e}_{1}\right)
$$

Notice that

$$
\left|\boldsymbol{\lambda}_{2}\right| \leq c\left|\boldsymbol{\mu}_{0}\right| \leq c|\varepsilon|, \quad\left|\boldsymbol{\lambda}_{1}\right| \leq c, \quad|\mathbf{W}-\mathbf{I}| \leq c|\varepsilon|
$$

which yields the estimate

$$
\left|\overline{\left\{\mathbf{W} \boldsymbol{\lambda}_{1}\right\} \cdot \mathbf{e}_{1}}-1\right| \leq c|\varepsilon|
$$

Next, estimate (7.15) and estimates (7.25) imply the inequality $\| \mathbf{S} \boldsymbol{\mu}_{1}+$ $\mathbf{T}^{\top} \boldsymbol{\lambda}_{1} \|_{\sigma / 2,0} \leq c$. Hence we can apply Lemma E.2 to equation (8.32) to obtain $\left\|\chi_{1}^{*}\right\|_{\sigma / 4,0} \leq c$. On the other hand, we have $\left|\nabla w_{1} *\right| \leq c|\varepsilon|$. It follows that

$$
\left|\overline{\chi_{1} \cdot \nabla w_{1}^{*}}\right|=\left|\overline{\chi_{1}^{*} \cdot \nabla w_{1}^{*}}\right| \leq c|\varepsilon| .
$$

Combining (8.37), (8.38) and recalling expression (8.35e) we arrive at the estimate

$$
\left|\mathcal{C}^{-1}-1\right| \leq c|\varepsilon|
$$


Choosing $|\varepsilon| \leq \varepsilon_{0}$ sufficiently small we obtain the desired estimate (8.31) for $\mathcal{C}$. We are now in a position to estimate the functions $g_{1}$ and $\mathbf{g}_{2}$ in (8.36). It follows from estimate (8.24) for $p_{1}$ and estimate (8.31) for $\mathcal{C}$ that

$\left\|g_{1}\right\|_{\sigma / 2,0} \leq c\left|p_{1}\right||| w_{1}^{*}\left\|_{\sigma / 2,0} \leq c\left|\boldsymbol{\mu}_{0}\right||\varepsilon|, \quad\right\| g_{2}\left\|_{\sigma / 2,0} \leq c\left|p_{1}\right||| \mathbf{W}-\mathbf{I}\right\|_{\sigma / 2,0} \leq c\left|\boldsymbol{\mu}_{0}\right||\varepsilon|$

Applying Theorem 8.1 to the problem (8.5) with the right hand sides (8.36) we arrive at the estimate

$$
\|\boldsymbol{\mu}\|_{\sigma / 4,0}+\|\boldsymbol{\lambda}\|_{\sigma / 4,0} \leq c\left|\varepsilon \| \boldsymbol{\mu}_{0}\right|
$$

Finally set

$$
\boldsymbol{\mu}_{\varepsilon}=\boldsymbol{\mu}+(\mathcal{C}-1) \boldsymbol{\mu}_{1}+\boldsymbol{\mu}_{2}, \quad \boldsymbol{\lambda}_{\varepsilon}=\boldsymbol{\lambda}-(\mathcal{C}-1)\left(\mathbf{t}_{0} \cdot \boldsymbol{\mu}_{0}\right) \mathbf{e}_{2}+\mathcal{C} \boldsymbol{\lambda}_{2}
$$

It remains to note that decomposition (8.30) follows from decompositions (8.23) and (8.34), and estimates (8.31) for $\boldsymbol{\mu}_{\varepsilon}$ and $\boldsymbol{\lambda}_{\varepsilon}$ follow from estimate (8.31) for $\mathcal{C}$, estimate (8.39) for $\boldsymbol{\mu}$ and $\boldsymbol{\lambda}$ and estimates (8.24) for $\boldsymbol{\mu}_{i}$ and $\boldsymbol{\lambda}_{i}$.

\section{Quadratic form}

Let $\boldsymbol{\mu}^{(i)}, \boldsymbol{\lambda}^{(i)}$, given by Theorems 8.2 and 8.4 , be solutions to problems (7.25) and (7.26). We define the quadratic form $L=\left(L_{i j}\right), 1 \leq i, j \leq 2$, as follows

$$
L_{i j}=\int_{\mathbb{T}^{n-1}}\left\{\mathbf{S} \boldsymbol{\mu}^{(i)} \cdot \boldsymbol{\mu}^{(j)}-\left(\mathbf{J} \boldsymbol{\partial} \boldsymbol{\lambda}^{(i)}+\boldsymbol{\Omega} \boldsymbol{\lambda}^{(i)}\right) \cdot \boldsymbol{\lambda}^{(j)}\right\} d \boldsymbol{\xi} .
$$

This form plays the key role in our analysis of the bifurcation equation (4.7). As it will be shown in the next section, the derivatives of the action functional are represented in the terms of this form. The following theorem, which is the main result of this section, describe the properties of the quadratic form (9.1).

Theorem 9.1. Under the assumptions of of Theorem 8.1, there exists $\varepsilon_{0}>0$ with the following property. For every $|\varepsilon| \leq \varepsilon_{0}$, the elements of the quadratic form $L$ satisfy the inequalities

$$
\begin{array}{r}
\left|L_{12}\right| \leq c|\varepsilon|, \quad\left|L_{11}\right| \leq c, \\
c^{-1}\left|w_{1}^{*}\right|_{-1}^{2} \leq\left|L_{22}\right| \leq c\left\|w_{1}^{*}\right\|_{\sigma / 2,0}^{2} \leq c|\varepsilon|^{2} .
\end{array}
$$


If $k=0$, then

$$
\left|L_{11}-(2 \pi)^{n-1} \mathbf{K}_{0} \boldsymbol{\mu}_{0} \cdot \boldsymbol{\mu}_{0}\right| \leq c|\varepsilon|\left|\boldsymbol{\mu}_{0}\right|^{2}, \quad\left|L_{12}\right| \leq c|\varepsilon|\left|\boldsymbol{\mu}_{0}\right| .
$$

Here the strictly positive constant $c$ is independent of $\alpha, k$, and $\varepsilon$, the vector $\boldsymbol{\mu}_{0}$ is given by (8.21).

Proof. We begin with the observation that

$$
\begin{aligned}
\left|L_{i j}\right| \leq c\left(\left|\boldsymbol{\mu}^{(i)}\right|_{0}\left|\boldsymbol{\mu}^{(j)}\right|_{0}+\right. & \left.\left|\boldsymbol{\lambda}^{(i)}\right|_{1}\left|\boldsymbol{\lambda}^{(j)}\right|_{0}\right) \\
& \leq c\left(\left\|\boldsymbol{\mu}^{(i)}\right\|_{\sigma / 4,0}\left\|\boldsymbol{\mu}^{(j)}\right\|_{\sigma / 4,0}+\left\|\boldsymbol{\lambda}^{(i)}\right\|_{\sigma / 4,0}\left|\boldsymbol{\lambda}^{(j)}\right|_{\sigma / 4,0}\right) .
\end{aligned}
$$

Therefore, estimates of $\left|L_{i j}\right|$ from above in (9.3) obviously follows from estimates (8.12) and (8.29) in Theorems 8.2 and 8.4. Let us estimate $L_{22}$ from below. It follows from the expressions (2.6) and (2.9) for $\mathbf{J}$ and $\boldsymbol{\Omega}$ that

$$
L_{22}=\int_{\mathbb{T}^{n-1}}\left\{\mathbf{S} \boldsymbol{\mu}^{(2)} \cdot \boldsymbol{\mu}^{(2)}+k \lambda_{1}^{(2)^{2}}-\lambda_{2}^{(2)^{2}}-\lambda_{1}^{(2)} \boldsymbol{\partial} \lambda_{2}^{(2)}+\lambda_{2}^{(2)} \boldsymbol{\partial} \lambda_{1}^{(2)}\right\} d \boldsymbol{\xi} .
$$

Integrating by parts gives

$$
L_{22}=\int_{\mathbb{T}^{n-1}}\left\{\mathbf{S} \boldsymbol{\mu}^{(2)} \cdot \boldsymbol{\mu}^{(2)}+k \lambda_{1}^{(2)^{2}}-\lambda_{2}^{(2)^{2}}+2 \lambda_{2}^{(2)} \boldsymbol{\partial} \lambda_{1}^{(2)}\right\} d \boldsymbol{\xi} .
$$

Next, multiplying both sides of equation (7.26b) by $\mathbf{e}_{2}$ we obtain

$$
-\boldsymbol{\partial} \lambda_{1}^{(2)}+\lambda_{2}^{(2)}+\mathbf{t}_{2} \cdot \boldsymbol{\mu}^{(2)}=-\left(p^{(2)}+w_{1}^{*}\right) \mathbf{W}^{\top} \mathbf{e}_{1} \cdot \mathbf{e}_{2} .
$$

It follows that

$$
2 \boldsymbol{\partial} \lambda_{1}^{(2)}-\lambda_{2}^{(2)}=\lambda_{2}^{(2)}+2 \mathbf{t}_{2} \cdot \boldsymbol{\mu}^{(2)}+2\left(p^{(2)}+w_{1}^{*}\right) \mathbf{W}^{\top} \mathbf{e}_{1} \cdot \mathbf{e}_{2} .
$$

Substituting this expression into (9.4) and noting that $\mathbf{W}^{\top} \mathbf{e}_{1} \cdot \mathbf{e}_{2}=W_{12}$ we arrive at the identity

$$
\begin{aligned}
L_{22}=\int_{\mathbb{T}^{n-1}}\left\{\mathbf{S} \boldsymbol{\mu}^{(2)} \cdot \boldsymbol{\mu}^{(2)}+\right. & k \lambda_{1}^{(2)^{2}}+\lambda_{2}^{(2)^{2}} \\
& \left.+2 \lambda_{2}^{(2)}\left(\mathbf{t}_{2} \cdot \boldsymbol{\mu}^{(2)}+2\left(p^{(2)}+w_{1}^{*}\right)\right) W_{12}\right\} d \boldsymbol{\xi} .
\end{aligned}
$$

Introduce the denotation $\varkappa=W_{12} W_{11}^{-1}$. Multiplying both side of (7.26b) by $\varkappa \mathbf{e}_{1}$ we arrive at

$$
-\left(p^{(2)}+w_{1}^{*}\right) W_{12}=-\varkappa\left(p^{(2)}+w_{1}^{*}\right) W_{11}=\varkappa\left(\boldsymbol{\partial} \lambda_{2}^{(2)}-k \lambda_{1}^{(2)}+\mathbf{t}_{1} \cdot \boldsymbol{\mu}^{(2)}\right)
$$


Thus we get

$$
\begin{aligned}
& \int_{\mathbb{T}^{n-1}} 2 \lambda_{2}^{(2)}\left(\mathbf{t}_{2} \cdot \boldsymbol{\mu}^{(2)}+2\left(p^{(2)}+w_{1}^{*}\right)\right) W_{12} d \boldsymbol{\xi}= \\
& \int_{\mathbb{T}^{n-1}}\left\{2 \lambda_{2}^{(2)}\left(\mathbf{t}_{2} \cdot \boldsymbol{\mu}^{(2)}\right)-\varkappa \lambda_{2}^{(2)} \boldsymbol{\partial} \lambda_{2}^{(2)}+\varkappa k \lambda_{2}^{(2)} \lambda_{1}^{(2)}-\varkappa \lambda_{2}^{(2)}\left(\mathbf{t}_{1} \cdot \boldsymbol{\mu}^{(2)}\right)\right\} d \boldsymbol{\xi}= \\
& \int_{\mathbb{T}^{n-1}}\left\{2 \lambda_{2}^{(2)}\left(\mathbf{t}_{2} \cdot \boldsymbol{\mu}^{(2)}\right)+\frac{1}{2} \boldsymbol{\partial} \varkappa\left(\lambda_{2}^{(2)}\right)^{2}+\varkappa k \lambda_{2}^{(2)} \lambda_{1}^{(2)}-\varkappa \lambda_{2}^{(2)}\left(\mathbf{t}_{1} \cdot \boldsymbol{\mu}^{(2)}\right)\right\} d \boldsymbol{\xi}:=\mathbf{I}_{0} .
\end{aligned}
$$

Let us estimate the quantity $\mathbf{I}_{0}$. It follows from estimates (7.4) for the matrix $\mathbf{W}$ in Theorem 7.1 and estimate (7.15) for the matrix $\mathbf{T}^{\top}=\left[\mathbf{t}_{1}, \mathbf{t}_{2}\right]$ in Lemma 7.2 that

$$
|\varkappa|+|\boldsymbol{\partial} \varkappa| \leq c|\varepsilon|, \quad\left|\mathbf{t}_{2}\right| \leq c, \quad\left|\mathbf{t}_{1}\right| \leq c|\varepsilon| .
$$

Thus we get

$$
\begin{aligned}
\left|\mathbf{I}_{0}\right| \leq c \int_{\mathbb{T}^{n-1}}\left|\lambda_{2}^{(2)}\right|\left|\boldsymbol{\mu}^{(2)}\right| d \boldsymbol{\xi}+c|\varepsilon| \int_{\mathbb{T}^{n-1}}\left(\left|\lambda_{2}^{(2)}\right|^{2}+k\left|\lambda_{1}^{(2)}\right|^{2}\right) d \boldsymbol{\xi} \leq \\
c|\varepsilon| \int_{\mathbb{T}^{n-1}}\left(\left|\lambda_{2}^{(2)}\right|^{2}+k\left|\lambda_{1}^{(2)}\right|^{2}\right) d \boldsymbol{\xi}+|\varepsilon|^{-1} \int_{\mathbb{T}^{n-1}}\left|\boldsymbol{\mu}^{(2)}\right|^{2} d \boldsymbol{\xi}
\end{aligned}
$$

On the other hand, we have

$$
L_{22}=\int_{\mathbb{T}^{n-1}}\left\{\mathbf{S} \boldsymbol{\mu}^{(2)} \cdot \boldsymbol{\mu}^{(2)}+k \lambda_{1}^{(2)^{2}}+\lambda_{2}^{(2)^{2}}\right\}+\mathbf{I}_{0} .
$$

It follows from this and (9.7) that

$$
L_{22} \geq(1-c|\varepsilon|)\left|\lambda_{2}^{(2)}\right|_{0}^{2}+k\left|\lambda_{1}^{(2)}\right|^{2}-c|\varepsilon|^{-1}\left|\boldsymbol{\mu}^{(2)}\right|_{0}^{2}
$$

Applying estimates (8.12) and (8.13) in Theorem 8.2 we arrive at the inequality

$$
L_{22} \geq c^{-1}(1-c|\varepsilon|)\left|w_{1}^{*}\right|_{-1}^{2}-c|\varepsilon|\left|w_{1}^{*}\right|_{-1}^{2} .
$$

Choosing $\varepsilon$ sufficiently small we obtain the desired estimate (9.2). It remains to prove inequalities (9.3). Notice that for $k=0$, the equality $\mathbf{\Omega a}=a_{2} \mathbf{e}_{2}$ holds for every vector a. Substituting decomposition (8.30) into the expression (9.1) we arrive at the identity

$$
L_{11}=\int_{\mathbb{T}^{n-1}}\left\{\mathbf{S}_{0} \boldsymbol{\mu}_{0} \cdot \boldsymbol{\mu}_{0}-\left(\boldsymbol{\mu}_{0} \cdot \mathbf{t}_{0}\right)^{2}\right\} d \boldsymbol{\xi}+\mathbf{I}_{1},
$$


where

$$
\begin{aligned}
\mathbf{I}_{1}=\int_{\mathbb{T}^{n-1}}\left(\mathbf{S}-\mathbf{S}_{0}\right) \boldsymbol{\mu}_{0} \cdot \boldsymbol{\mu}_{0} d \boldsymbol{\xi}+\int_{\mathbb{T}^{n-1}} \mathbf{S} \boldsymbol{\mu}_{\varepsilon} \cdot\left(2 \boldsymbol{\mu}_{0}+\boldsymbol{\mu}_{\varepsilon}\right) d \boldsymbol{\xi} \\
-\int_{\mathbb{T}^{n-1}}\left\{\left(\mathbf{J} \boldsymbol{\partial} \boldsymbol{\lambda}_{\varepsilon}+\boldsymbol{\Omega} \boldsymbol{\lambda}_{\varepsilon}\right) \cdot \boldsymbol{\lambda}_{\varepsilon}+2\left(\boldsymbol{\mu}_{0} \cdot \mathbf{t}_{0}\right) \lambda_{\varepsilon, 2}\right\}, d \boldsymbol{\xi}
\end{aligned}
$$

Estimates (7.15) for the matrices $\mathbf{S}$ and $\mathbf{T}^{\top}=\left[\mathbf{t}_{1}, \mathbf{t}_{2}\right]$ in Lemma 7.2 imply

$$
\left|\mathbf{S}-\mathbf{S}_{0}\right| \leq c|\varepsilon|, \quad\left|\mathbf{t}_{2}\right| \leq c
$$

In its turn, estimates (8.31) in Theorem 8.4 imply

$$
\left|\boldsymbol{\mu}_{\varepsilon}\right|+\left|\boldsymbol{\partial} \boldsymbol{\mu}_{\varepsilon}+\right|\left|\boldsymbol{\lambda}_{\varepsilon}\right| \leq c|\varepsilon|\left|\boldsymbol{\mu}_{0}\right| .
$$

Combining these estimates we finally obtain

$$
\left|\mathbf{I}_{1}\right| \leq c|\varepsilon|\left|\boldsymbol{\mu}_{0}\right|^{2}
$$

Next notice that

$$
\int_{\mathbb{T}^{n-1}}\left\{\mathbf{S}_{0} \boldsymbol{\mu}_{0} \cdot \boldsymbol{\mu}_{0}-\left(\boldsymbol{\mu}_{0} \cdot \mathbf{t}_{0}\right)^{2}\right\} d \boldsymbol{\xi}=(2 \pi)^{n-1} \mathbf{K}_{0} \boldsymbol{\mu}_{0} \cdot \boldsymbol{\mu}_{0}
$$

Substituting this equality into (9.8) and using inequality (9.10) we obtain estimate (9.3) for $L_{11}$. It remains to estimate $L_{12}$. Since $k=0$ we have

$$
L_{12}=\int_{\mathbb{T}^{n-1}}\left\{\mathbf{S} \boldsymbol{\mu}^{(1)} \cdot \boldsymbol{\mu}^{(2)}-\left(\mathbf{J} \boldsymbol{\partial} \boldsymbol{\lambda}^{(2)}+\boldsymbol{\Omega} \boldsymbol{\lambda}^{(2)}\right) \cdot\left(\boldsymbol{\lambda}^{(1)}-\mathcal{C} \mathbf{e}_{1}\right)\right\} d \boldsymbol{\xi} .
$$

In view of estimates (8.31) in Theorem 8.4, we have

$$
\left|\boldsymbol{\mu}^{(1)}\right| \leq c\left|\boldsymbol{\mu}_{0}, \quad\right| \boldsymbol{\lambda}^{(1)}-\mathcal{C} \mathbf{e}_{1}|| \leq c\left|\boldsymbol{\mu}_{0}\right|, \quad\left|\boldsymbol{\partial} \boldsymbol{\lambda}^{(1)}\right| \leq c\left|\boldsymbol{\mu}_{0}\right|
$$

On the other hand, estimates (8.12) in Theorem 8.4 yield

$$
\left\|\boldsymbol{\lambda}^{(2)}\right\|_{\sigma / 4,0}+\left\|\boldsymbol{m} \boldsymbol{u}^{(2)}\right\|_{\sigma / 4,0}+\left|p^{(2)}\right| \leq c|\varepsilon| .
$$

This result and the expression (9.11) for $L_{12}$ lead to the estimate $\left|L_{12}\right| \leq$ $c|\varepsilon|\left|\boldsymbol{\mu}_{0}\right|$. This completes the proof of Theorem 


\section{Action functional. Proof of Theorem 2.2}

Theorem 7.1 constitutes the existence and uniqueness of the solution

$$
\begin{array}{r}
\mathfrak{u}=(\boldsymbol{\varphi}(\alpha, k), e(\alpha, k)), m(\alpha, k), M(\alpha, k), \\
\boldsymbol{\varphi}(\alpha, k)=\left(\boldsymbol{\beta}, \varphi_{0}, \mathbf{u}, \mathbf{w}, W_{11}, W_{12}, W_{21}\right) .
\end{array}
$$

of the main operator equation (4.16) for every $(\alpha, k) \in \mathbb{T} \times[0,1]$ and for all sufficiently small $\varepsilon$. This means that the vector

$$
\Theta(\varphi)=\left(\mathbf{u}, \mathbf{v}, \mathbf{w}, \mathbf{V}, \boldsymbol{\Lambda}, \mathbf{W}, \mathbf{R}_{i}\right)
$$

with the components $\mathbf{v}, \mathbf{V}, \mathbf{W}$, and $\mathbf{R}_{i}$ defined by relations (3.8), determines the canonical mapping $\boldsymbol{\vartheta}$ given by (3.1). By the definition of the operator $\Phi$, this mapping puts the modified Hamiltonian $H_{m}$ into the normal form (2.8). By virtue of Definition 2.1 of the normal form, the modified Hamiltonian has a weakly hyperbolic invariant torus. In the phase space, these torus has the the parametric representation

$$
\mathbf{x}(\boldsymbol{\xi})=\boldsymbol{\xi}+\mathbf{u}(\boldsymbol{\xi}), \quad \mathbf{y}(\boldsymbol{\xi})=\mathbf{v}(\boldsymbol{\xi}), \quad \mathbf{z}=\mathbf{w}(\boldsymbol{\xi}), \quad \boldsymbol{\xi} \in \mathbb{T}^{n-1},
$$

in which $\mathbf{u}, \mathbf{w}$ are the components of the vector $\boldsymbol{\varphi}(\alpha, k)$, and the component $\mathbf{v}$ of the vector $\boldsymbol{\Theta}$ is defined by the relation (3.7), i.e.,

$$
\mathbf{v}=\boldsymbol{\beta}+\mathbf{V}\left(\nabla \varphi_{0}-w_{2} \nabla w_{1}\right), \quad \mathbf{V}=\left(\mathbf{I}+\mathbf{u}^{\prime}\right)^{-\top} .
$$

By virtue of Theorem 6.2, the main operator equation (4.16) has an analytic periodic solution for all $(\alpha, k) \in \mathbb{T} \times[0,1]$ and for all sufficiently small $\varepsilon$. In other words, the modified Hamiltonian has the two-parametric family of weakly hyperbolic invariant tori labeled by $(\alpha, k)$. Notice that $H_{m}(\mathbf{x}, \mathbf{y}, \mathbf{z})=$ $H(\mathbf{x}, \mathbf{y}, \mathbf{z})+m z_{1}+2^{-1} M z_{1}^{2}$, where $m=m(\alpha, k), M=M(\alpha, k)$ are the components of the vector $\mathfrak{u}$ satisfying (4.16). Therefore, the hamiltonian $H$ has a weakly hyperbolic invariant torus if and only if

$$
m(\alpha, k)=0, M(\alpha, k)=0
$$

Relations (10.5) give the system of two scalar bifurcation equations for $(\alpha, k) \in$ $\mathbb{T}^{1} \times[0,1]$. The difficulty is that the scalars $(m, M)$ are the integral part of a solution to the complicated operator equation, and we know nothing about their properties. In order to cope with this problem, we notice that every 
Hamiltonian system has a variational formulation, and its solutions are critical points of the action functional. For quasi-periodic solutions, the action functional can be written as the Perceval functional, see [16],

$$
\Psi=\int_{\mathbf{T}^{n-1}}\left((\boldsymbol{\omega}+\boldsymbol{\partial u}) \mathbf{v}+w_{2} \boldsymbol{\partial} w_{1}-H(\boldsymbol{\xi}+\mathbf{u}, \mathbf{v}, \mathbf{w})\right) d \boldsymbol{\xi}
$$

In view of Theorem 7.1, the mappings

$$
\begin{array}{r}
\mathbb{R} \times[0,1] \ni(\alpha, k) \rightarrow \mathbf{u}(\alpha, k) \in \mathcal{A}_{\sigma / 4, d}, \\
\mathbb{R} \times[0,1] \ni(\alpha, k) \rightarrow \mathbf{v}(\alpha, k) \in \mathcal{A}_{\sigma / 4, d}, \\
\mathbb{R} \times[0,1] \ni(\alpha, k) \rightarrow \mathbf{w}(\alpha, k)-\alpha \mathbf{e}_{1} \in \mathcal{A}_{\sigma / 2, d}, \\
\mathbb{R} \times[0,1] \ni(\alpha, k) \rightarrow \mathbf{W}(\alpha, k) \in \mathcal{A}_{\sigma / 2, d}, \\
\mathbb{R} \times[0,1] \ni(\alpha, k) \rightarrow M(\alpha, k) \in \mathbb{R}^{1}, \\
\mathbb{R} \times[0,1] \ni(\alpha, k) \rightarrow m(\alpha, k)+\alpha M(\alpha, k) \in \mathbb{R}^{1},
\end{array}
$$

are continuously differentiable and $2 \pi$-periodic in $\alpha$. Moreover, they are analytic in $\mathbb{R} \times(0,1)$ and satisfy the inequalities

$$
\begin{aligned}
\left\|\partial_{\alpha}^{r} \mathbf{u}\right\|_{\sigma / 2, d}+\left\|\partial_{\alpha}^{r} \mathbf{v}\right\|_{\sigma / 2, d} & \leq c(r)|\varepsilon| \\
\left\|\partial_{\alpha}^{r}\left(\mathbf{w}-\alpha \mathbf{e}_{1}\right)\right\|_{\sigma / 2, d}++\left\|\partial_{\alpha}^{r}(\mathbf{W}-\mathbf{I})\right\|_{\sigma / 2, d} & \leq c(r)|\varepsilon|, \\
\left|\partial_{\alpha}^{r} \boldsymbol{\beta}\right|+\left|\partial_{\alpha}^{r}(m+\alpha M)\right|+\left|\partial_{\alpha}^{r}(M+k)\right| & \leq c(r)|\varepsilon|,
\end{aligned}
$$

and

$$
\begin{array}{r}
\left\|\partial_{\alpha}^{r} \partial_{k} \mathbf{u}\right\|_{\sigma / 2, d}+\left\|\partial_{\alpha}^{r} \partial_{k} \mathbf{v}\right\|_{\sigma / 2, d} \leq c|\varepsilon| \\
\left\|\partial_{\alpha}^{r} \partial_{k}\left(\mathbf{w}-\alpha \mathbf{e}_{1}\right)\right\|_{\sigma / 2, d}+\left\|\partial_{\alpha}^{r} \partial_{k}(\mathbf{W}-\mathbf{I})\right\|_{\sigma / 2, d} \leq c|\varepsilon| \\
\left|\partial_{\alpha}^{r} \partial_{k}(m+\alpha M)\right|+\left|\partial_{\alpha}^{r} \partial_{k}(M+k)\right| \leq c(r)|\varepsilon|
\end{array}
$$

where $r \geq 0$ is an arbitrary integer, the constant $c$ is independent of $\alpha, k$, and $\varepsilon$. It follows from this that the mapping $\Psi$ is a function of the variables $(\alpha, k)$, which belong to the Banach space $C^{1}\left([0,1] ; \mathcal{A}_{\sigma / 2,0}\right)$. In particular, $\Psi$ and $\partial_{k} \Psi$ are continuous in $k$ and analytic in $\alpha$ "uniformly" with respect to $k \in[0,1]$.

The following Theorem constitutes the relations between $\Psi, m, M$ and the quadratic form $\left(L_{i j}\right)$. 
Theorem 10.1. Under the above assumptions, there is $\varepsilon_{0}>0$ with the properties. For every $|\varepsilon| \leq \varepsilon_{0}$ the derivatives of the function $\Psi$ admit the representation

$$
\begin{gathered}
\partial_{\alpha} \Psi(\alpha, k)=(2 \pi)^{n-1}(m+\alpha M)+\varsigma_{1} M \\
\partial_{k} \Psi(\alpha, k)=M \partial_{k} M L_{22}, \\
\partial_{\alpha}^{2} \Psi(\alpha, k)=(2 \pi)^{n-1} M+\varsigma_{2} M+L_{11}+2 L_{12} \partial_{\alpha} M+L_{22}\left(\partial_{\alpha} M\right)^{2} .
\end{gathered}
$$

Here $L_{i j}$ are given by (9.1), the quantities $\varsigma_{i}(\alpha, k)$ satisfy the inequalities

$$
\left|\varsigma_{i}\right|+\left|\partial_{\alpha} \varsigma_{i}\right| \leq c|\varepsilon|^{2}
$$

Proof. Differentiation (10.6) with respect to the variable $\tau, \tau=\alpha, k$, and integrating by parts gives

$$
\begin{gathered}
\partial_{\tau} \Psi(\alpha, k)=\int_{\mathbf{T}^{n-1}}\left(\left(\boldsymbol{\omega}+\boldsymbol{\partial} \mathbf{u}-\nabla_{y} H(\boldsymbol{\xi}+\mathbf{u}, \mathbf{v}, \mathbf{w})\right) \cdot \partial_{\tau} \mathbf{v} d \boldsymbol{\xi}\right. \\
-\int_{\mathbf{T}^{n-1}}\left(\boldsymbol{\partial} \mathbf{v}+\nabla_{x} H(\boldsymbol{\xi}+\mathbf{u}, \mathbf{v}, \mathbf{w})\right) \cdot \partial_{\tau} \mathbf{u} d \boldsymbol{\xi}+ \\
\quad \int_{\mathbf{T}^{n-1}}\left(\boldsymbol{\partial} w_{1}-\partial_{z_{2}} H(\boldsymbol{\xi}+\mathbf{u}, \mathbf{v}, \mathbf{w})\right) \cdot \partial_{\tau} w_{2} d \boldsymbol{\xi} \\
-\int_{\mathbf{T}^{n-1}}\left(\boldsymbol{\partial} w_{2}+\partial_{z_{1}} H(\boldsymbol{\xi}+\mathbf{u}, \mathbf{v}, \mathbf{w})\right) \cdot \partial_{\tau} w_{1} d \boldsymbol{\xi}
\end{gathered}
$$

Next notice that

$$
\begin{array}{r}
\nabla_{y} H=\nabla_{y} H_{m}, \quad \nabla_{x} H=\nabla_{x} H_{m}, \quad \partial_{z_{2}} H=\partial_{z_{2}} H_{m}, \\
\left.\left.\partial_{z_{1}} H(\boldsymbol{\xi}+\mathbf{u}, \mathbf{v}, \mathbf{w})\right)+m+M w_{1}=\partial_{z_{1}} H_{m}(\boldsymbol{\xi}+\mathbf{u}, \mathbf{v}, \mathbf{w})\right) .
\end{array}
$$

Recall that the vector $\varphi$ given by (10.1) serves as a solution to operator equation (4.16). From this and Lemma 4.1 we conclude that $\mathbf{u}, \mathbf{v}$, and $\mathbf{w}$ satisfy equations (4.11), i.e.,

$$
\begin{array}{r}
\boldsymbol{\omega}+\boldsymbol{\partial} \mathbf{u}=\nabla_{y} H_{m}(\boldsymbol{\xi}+\mathbf{u}, \mathbf{v}, \mathbf{w}), \\
\boldsymbol{\partial} \mathbf{v}=-\nabla_{x} H_{m}(\boldsymbol{\xi}+\mathbf{u}, \mathbf{v}, \mathbf{w}), \quad \boldsymbol{\partial} \mathbf{w}=\mathbf{J} \nabla_{z} H_{m}(\boldsymbol{\xi}+\mathbf{u}, \mathbf{v}, \mathbf{w}) .
\end{array}
$$

Substituting these equality along with (10.15) into (10.14) we arrive at the identity

$$
\partial_{\tau} \Psi(\alpha, k)=\int_{\mathbf{T}^{n-1}}\left(m+w_{1} M\right) \partial_{\tau} w_{1} d \boldsymbol{\xi}, \quad \tau=\alpha, k .
$$


Recall the denotations

$$
\overline{w_{1}}=(2 \pi)^{-n+1} \int_{\mathbb{T}^{n-1}} w_{1} d \boldsymbol{\xi}, \quad w_{1}^{*}=w_{1}-\overline{w_{1}} .
$$

Notice that

$$
w_{1}=\alpha+w_{1}^{*}, \quad \partial_{\alpha} w_{1}=1+\partial_{\alpha} w_{1}^{*}, \quad \partial_{k} w_{1}=\partial_{k} w_{1}^{*}
$$

From this and (10.16) we obtain

$$
\begin{array}{r}
\partial_{\alpha} \Psi(\alpha, k)=(2 \pi)^{n-1}(m+\alpha M)+\varsigma_{1} M, \\
\partial_{k} \Psi(\alpha, k)=M \int_{\mathbb{T}^{n-1}} w_{1}^{*} \partial_{k} w_{1}^{*} d \boldsymbol{\xi},
\end{array}
$$

where

$$
\varsigma_{1}=\int_{\mathbb{T}^{n-1}} w_{1}^{*} \partial_{\alpha} w_{1}^{*} d \boldsymbol{\xi} .
$$

It obviously follows from estimates (10.8) that $\varsigma_{1}$ satisfies inequality (10.13). This leads to representation (10.10).

Let us prove representation (10.11). Recall formulae (7.10) which constitutes the linear algebraic relation between the vector field $\left(\partial_{\tau} \mathbf{u}, \partial_{\tau} \mathbf{v}, \partial_{\tau} \mathbf{w}\right)$ and the vector field $\left(\boldsymbol{\mu}^{(\tau)}, \boldsymbol{\lambda}^{(\tau)}, \boldsymbol{\chi}^{(\tau)}\right)$. In view of the Second Structure Theorem (3.2) this relation has the inverse given by (3.19). In particular, we have

$$
\partial_{\tau} \mathbf{w}=\mathbf{W} \boldsymbol{\lambda}^{(\tau)}+\chi_{i}^{(\tau)} \frac{\partial}{\partial \xi_{i}} \mathbf{w} .
$$

On the other hand, relation (17.24) in Corollary 7.3 yields

$$
\left(\boldsymbol{\mu}^{(k)}, \boldsymbol{\lambda}^{(k)}, \boldsymbol{\chi}^{(k)}\right)=\partial_{k} M\left(\boldsymbol{\mu}^{(2)}, \boldsymbol{\lambda}^{(2)}, \boldsymbol{\chi}^{(2)}\right)
$$

Substituting this relation into identity (10.18) with $\tau$ replaced by $k$, we obtain

$$
\partial_{k} w_{1}^{*}=\partial_{k} \mathbf{w} \cdot \mathbf{e}_{1}=\partial_{k} M\left\{\left(\mathbf{W}^{\top} \mathbf{e}_{1}\right) \cdot \boldsymbol{\lambda}^{(2)}+\nabla w_{1}^{*} \cdot \chi^{(2)}\right\} .
$$

It follows that

$$
\begin{gathered}
\int_{\mathbb{T}^{n-1}} w_{1}^{*} \partial_{k} w_{1}^{*} d \boldsymbol{\xi}=\int_{\mathbb{T}^{n-1}}\left(p^{(2)}+w_{1}^{*}\right) \partial_{k} w_{1}^{*} d \boldsymbol{\xi}= \\
\partial_{k} M \int_{\mathbb{T}^{n-1}}\left\{\left(p^{(2)}+w_{1}^{*}\right) \mathbf{W}^{\top} \mathbf{e}_{1}\right\} \cdot \boldsymbol{\lambda}^{(2)} d \boldsymbol{\xi}+\partial_{k} M \int_{\mathbb{T}^{n-1}}\left\{\left(p^{(2)}+w_{1}^{*}\right) \nabla w_{1}^{*}\right\} \cdot \boldsymbol{\chi}^{(2)} d \boldsymbol{\xi}
\end{gathered}
$$


Next, equations (7.26) imply the equalities

$$
\begin{gathered}
\left(p^{(2)}+w_{1}^{*}\right) \nabla w_{1}^{*}=-\boldsymbol{\partial} \boldsymbol{\mu}^{(2)} \\
\left(p^{(2)}+w_{1}^{*}\right) \mathbf{W}^{\top} \boldsymbol{e}_{1}=-\mathbf{J} \boldsymbol{\partial} \boldsymbol{\lambda}^{(2)}-\boldsymbol{\Omega} \boldsymbol{\lambda}^{(2)}-\mathbf{T} \boldsymbol{\mu}^{(2)} .
\end{gathered}
$$

Combining the obtained results we arrive at the identity

$$
\begin{aligned}
\int_{\mathbb{T}^{n-1}} w_{1}^{*} \partial_{k} w_{1}^{*} d \boldsymbol{\xi}=-\partial_{k} M \int_{\mathbb{T}^{n-1}}\left(\left(\mathbf{J} \boldsymbol{\partial} \boldsymbol{\lambda}^{(2)}\right.\right. \\
\left.\left.+\boldsymbol{\Omega} \boldsymbol{\lambda}^{(2)}+\mathbf{T} \boldsymbol{\mu}^{(2)}\right) \cdot \boldsymbol{\lambda}^{(2)}+\boldsymbol{\partial} \boldsymbol{\mu}^{(2)} \cdot \boldsymbol{\chi}^{(2)}\right) d \boldsymbol{\xi} .
\end{aligned}
$$

Equation (7.26c) yields the identity

$$
\begin{gathered}
-\partial_{k} M \int_{\mathbb{T}^{n-1}} \boldsymbol{\partial} \boldsymbol{\mu}^{(2)} \cdot \boldsymbol{\chi}^{(2)} d \boldsymbol{\xi}=\partial_{k} M \int_{\mathbb{T}^{n-1}} \boldsymbol{\partial} \boldsymbol{\mu}^{(2)} \cdot \boldsymbol{\chi}^{(2)} d \boldsymbol{\xi} \\
=\partial_{k} M \int_{\mathbb{T}^{n-1}}\left(\mathbf{S} \boldsymbol{\mu}^{(2)}+\mathbf{T}^{\top} \boldsymbol{\lambda}^{(2)}\right) \cdot \boldsymbol{\mu}^{(2)} d \boldsymbol{\xi}
\end{gathered}
$$

Substituting this equality into (10.20) we finally obtain

$$
\begin{array}{rl}
\int_{\mathbb{T}^{n-1}} w_{1}^{*} \partial_{k} w_{1}^{*} d \boldsymbol{\xi}=\partial_{k} & M \int_{\mathbb{T}^{n-1}}\left(\mathbf{S} \boldsymbol{\mu}^{(2)} \cdot \boldsymbol{\mu}^{(2)}\right. \\
& \left.-\left(\mathbf{J} \boldsymbol{\partial} \boldsymbol{\lambda}^{(2)}+\boldsymbol{\Omega} \boldsymbol{\lambda}^{(2)}\right) \cdot \boldsymbol{\lambda}^{(2)}\right) d \boldsymbol{\xi}=\partial_{k} M L_{22}
\end{array}
$$

It remains to notice that desired identity (10.11) obviously follows from (10.21) and identity (10.17).

Our next task is to prove identity (10.12). Differentiating identity (10.10) with respect to $\alpha$ and noting that $\overline{\partial_{\alpha}^{2} w_{1}}=0$ we obtain

$$
\partial_{\alpha}^{2} \Psi(\alpha, k)=M \int_{\mathbb{T}^{n-1}}\left(\left(\partial_{\alpha} w_{1}\right)^{2}+w_{1}^{*} \partial_{\alpha}^{2} w_{1}^{*}\right) d \boldsymbol{\xi}+R,
$$

where

$$
R=\int_{\mathbb{T}^{n-1}}\left(\partial_{\alpha} m+w_{1} \partial_{\alpha} M\right) d \boldsymbol{\xi}
$$

Since $\left(\partial_{\alpha} w_{1}\right)^{2}=1+2 \partial_{\alpha} w_{1}^{*}+\left(\partial_{\alpha} w_{1}^{*}\right)^{2}$, we have

$$
\int_{\mathbb{T}^{n-1}}\left(\left(\partial_{\alpha} w_{1}\right)^{2}+w_{1}^{*} \partial_{\alpha}^{2} w_{1}^{*}\right) d \boldsymbol{\xi}=(2 \pi)^{n-1}+\varsigma_{2},
$$


where

$$
\varsigma_{2}=\int_{\mathbb{T}^{n-1}}\left(\left(\partial_{\alpha} w_{1}^{*}\right)^{2}+w_{1}^{*} \partial_{\alpha}^{2} w_{1}^{*}\right) d \boldsymbol{\xi}
$$

Estimate (10.8) implies that $\varsigma_{2}$ satisfies inequality (10.13). It remains to calculate $R$. Equality (7.11) yields

$$
\partial_{\alpha} m+w_{1} \partial_{\alpha} M=\partial_{\alpha} m+\alpha \partial_{\alpha} M+\partial_{\alpha} M w_{1}^{*}=p^{(\alpha)}+\partial_{\alpha} M w_{1}^{*}
$$

Thus we get

$$
R=\int_{\mathbb{T}^{n-1}}\left(p^{(\alpha)}+\partial_{\alpha} M w_{1}^{*}\right) \partial_{\alpha} w_{1} d \boldsymbol{\xi} .
$$

Next, relation (10.18) with $\tau=\alpha$ gives the identity

$$
\partial_{\alpha} w_{1}=\partial_{\alpha} \mathbf{w} \cdot \mathbf{e}_{1}=\mathbf{W}^{\top} \mathbf{e}_{1} \cdot \boldsymbol{\lambda}^{(\alpha)}+\chi^{(\alpha)} \cdot \nabla \mathbf{w}_{1}^{*}
$$

Substituting this result into (10.26) we arrive at the identity

$$
\begin{aligned}
R=\int_{\mathbb{T}^{n-1}}\left(p^{(\alpha)}+\partial_{\alpha} M w_{1}^{*}\right) \mathbf{W}^{\top} & \mathbf{e}_{1} \cdot \boldsymbol{\lambda}^{(\alpha)} d \boldsymbol{\xi} \\
& +\int_{\mathbb{T}^{n-1}}\left(p^{(\alpha)}+\partial_{\alpha} M w_{1}^{*}\right) \nabla w_{1}^{*} \cdot \chi^{(\alpha)} d \boldsymbol{\xi} .
\end{aligned}
$$

Next, equations (7.13a) and (7.13b) in Lemma 7.2 imply the equalities

$$
\begin{gathered}
\left(p^{(\alpha)}+w_{1}^{*}\right) \nabla w_{1}^{*}=-\boldsymbol{\partial} \boldsymbol{\mu}^{(\alpha)} \\
\left(p^{(\alpha)}+w_{1}^{*}\right) \mathbf{W}^{\top} \mathbf{e}_{1}=-\mathbf{J} \boldsymbol{\partial} \boldsymbol{\lambda}^{(\alpha)}-\boldsymbol{\Omega} \boldsymbol{\lambda}^{(\alpha)}-\mathbf{T} \boldsymbol{\mu}^{(\alpha)},
\end{gathered}
$$

Substituting these equalities into (10.26) we arrive at the identity

$$
R=-\int_{\mathbb{T}^{n-1}}\left(\left(\mathbf{J} \boldsymbol{\partial} \boldsymbol{\lambda}^{(\alpha)}+\Omega \boldsymbol{\lambda}^{(\alpha)}+\mathbf{T} \boldsymbol{\mu}^{(2)}\right) \cdot \boldsymbol{\lambda}^{(\alpha)}+\boldsymbol{\partial} \boldsymbol{\mu}^{(\alpha)} \cdot \boldsymbol{\chi}^{(\alpha)}\right) d \boldsymbol{\xi}
$$

On the other hand, equation (17.13c) with $\tau=\alpha$ implies

$$
\begin{gathered}
-\int_{\mathbb{T}^{n-1}} \boldsymbol{\partial} \boldsymbol{\mu}^{(\alpha)} \cdot \boldsymbol{\chi}^{(\alpha)} d \boldsymbol{\xi}=\int_{\mathbb{T}^{n-1}} \boldsymbol{\partial} \boldsymbol{\mu}^{(\alpha)} \cdot \boldsymbol{\chi}^{(\alpha)} d \boldsymbol{\xi} \\
=\int_{\mathbb{T}^{n-1}}\left(\mathbf{S} \boldsymbol{\mu}^{(\alpha)}+\mathbf{T}^{\top} \boldsymbol{\lambda}^{(\alpha)}\right) \cdot \boldsymbol{\mu}^{(2)} d \boldsymbol{\xi}
\end{gathered}
$$


Substituting this equality into (10.29) we obtain

$$
R=\int_{\mathbb{T}^{n-1}}\left(\mathbf{S} \boldsymbol{\mu}^{(\alpha)} \cdot \boldsymbol{\mu}^{(\alpha)}-\left(\mathbf{J} \boldsymbol{\partial} \boldsymbol{\lambda}^{(\alpha)}+\boldsymbol{\Omega} \boldsymbol{\lambda}^{(\alpha)}\right) \cdot \boldsymbol{\lambda}^{(\alpha)}\right) d \boldsymbol{\xi}=L_{22}
$$

Combining this relation with the equality

$$
\left(\boldsymbol{\mu}^{(\alpha)}, \boldsymbol{\lambda}^{(\alpha)}\right)=\left(\boldsymbol{\mu}^{(1)}, \boldsymbol{\lambda}^{(1)}\right)+\partial_{\alpha} M\left(\boldsymbol{\mu}^{(2)}, \boldsymbol{\lambda}^{(2)}\right)
$$

and recalling formula (9.1) for $L_{i j}$ we arrive at the expression for $R$,

$$
R=L_{11}+\partial_{\alpha} M 2 L_{12}+\left(\partial_{\alpha} M\right)^{2} L_{22},
$$

Substituting this expression and equality (10.24) into (10.22) we obtain desired identity (10.13). This completes the proof of Theorem 10.1.

The following proposition is a direct consequence of this theorem.

Proposition 10.2. Let all assumptions of Theorem 10.1 be satisfied and $k=0$. Then the third derivative of $\Psi$ admits the estimate

$$
\left|\partial_{\alpha}^{3} \Psi(\alpha, 0)-(2 \pi)^{n-1} \partial_{\alpha} M\right| \leq c|\varepsilon|\left(|M|+\left|\boldsymbol{\mu}_{0}\right|+\left|\partial_{\alpha} M\right|\right) .
$$

Proof. It follows from representation (10.12) in Theorem 10.1 that

$$
\partial_{\alpha}^{3} \Psi=(2 \pi)^{n-1} \partial_{\alpha} M+\varsigma_{2} \partial_{\alpha} M+\partial_{\alpha} \varsigma_{2} M+\mathbf{I}_{1}+\mathbf{I}_{2},
$$

where

$$
\begin{gathered}
\mathbf{I}_{1}=\partial_{\alpha} L_{11}+2 \partial_{\alpha} M \partial_{\alpha} L_{12}+\left(\partial_{\alpha} M\right)^{2} \partial_{\alpha} L_{22}, \\
\mathbf{I}_{2}=2 \partial_{\alpha}^{2} M L_{12}+2\left(\partial_{\alpha} M\right) \partial_{\alpha}^{2} M L_{22}
\end{gathered}
$$

The rest of the proof is based on the following

Lemma 10.3. Under the assumptions of Theorem 10.2,

$$
\left|\partial_{\alpha} L_{11}\right| \leq c|\varepsilon|\left|\boldsymbol{\mu}_{0}\right|, \quad\left|\partial_{\alpha} L_{12}\right|+\left|\partial_{\alpha} L_{22}\right| \leq c|\varepsilon| .
$$

Proof. First we estimates the vector fields $\boldsymbol{\mu}^{(i)}$ and $\boldsymbol{\lambda}^{(i)}, i=1,2$. We begin with the observation that relations in view of the identities (7.24),

$$
\begin{array}{r}
\left(\boldsymbol{\mu}^{(2)}, \boldsymbol{\lambda}^{(2)}\right)=\partial_{k} M^{-1}\left(\boldsymbol{\mu}^{(k)}, \boldsymbol{\lambda}^{(k)}\right), \\
\left(\boldsymbol{\mu}^{(1)}, \boldsymbol{\lambda}^{(1)}\right)=\left(\boldsymbol{\mu}^{(\alpha)}, \boldsymbol{\lambda}^{(\alpha)}\right)-\partial_{\alpha} M\left(\boldsymbol{\mu}^{(2)}, \boldsymbol{\lambda}^{(2)}\right) .
\end{array}
$$


In its turn, equalities (7.10) establish the following relations between the vector fields $\boldsymbol{\mu}^{(\tau)}, \boldsymbol{\lambda}^{(\tau)}$ and the derivatives of the vector fields $\mathbf{u}, \mathbf{v}$, and $\mathbf{w}$, i.e.,

$$
\begin{gathered}
\boldsymbol{\chi}^{(\tau)}=\mathbf{V}^{\top} \partial_{\tau} \mathbf{u} \\
\boldsymbol{\lambda}^{(\tau)}=\left(W^{-1} \partial_{\tau} \mathbf{w}-\chi_{i}^{(\tau)} \mathbf{W}^{-1} \frac{\partial}{\partial \xi_{i}} \mathbf{w}\right. \\
\boldsymbol{\mu}^{(\tau)}=\mathbf{V}^{-1}\left(\partial_{\tau} \mathbf{v}+\chi_{i}^{(\tau)} \frac{\partial}{\partial \xi_{i}} \mathbf{v}-\boldsymbol{\Lambda} \boldsymbol{\lambda}^{(\tau)}\right) .
\end{gathered}
$$

These identities along with estimates (10.8) and (10.9) imply

$$
\left\|\partial_{\alpha} \boldsymbol{\mu}^{(\tau)}\right\|_{\sigma / 4,0}+\left\|\partial_{\alpha} \boldsymbol{\lambda}^{(\tau)}\right\|_{\sigma / 4,0} \leq c|\varepsilon|, \quad \tau=\alpha, k .
$$

On the other hand, inequalities (10.9) yield

$$
\left|\partial_{k} M^{-1}\right| \leq(1-c|\varepsilon|)^{-1} \leq c, \quad\left|\partial_{k} \partial_{\alpha} M\right| \leq c|\varepsilon|
$$

Inequalities (10.37)-(10.38) and the identity

$$
\partial_{\alpha}\left(\boldsymbol{\mu}^{(2)}, \boldsymbol{\lambda}^{(2)}\right)=-\left(\partial_{k} M\right)^{-2} \partial_{\alpha} \partial_{k} M\left(\boldsymbol{\mu}^{(k)}, \boldsymbol{\lambda}^{(k)}\right)+\partial_{k} M^{-1} \partial_{\alpha}\left(\boldsymbol{\mu}^{(k)}, \boldsymbol{\lambda}^{(k)}\right)
$$

lead to the estimate

$$
\left|\partial_{\alpha}\left(\boldsymbol{\mu}^{(2)}, \boldsymbol{\lambda}^{(2)}\right)\right| \leq c|\varepsilon|
$$

Next, it follows from (10.36) that

$$
\begin{array}{ll}
\partial_{\alpha}\left(\boldsymbol{\mu}^{(1)}, \boldsymbol{\lambda}^{(1)}\right)=\partial_{\alpha}\left(\boldsymbol{\mu}^{(\alpha)}, \boldsymbol{\lambda}^{(\alpha)}\right)-\partial_{\alpha}^{2} M\left(\boldsymbol{\mu}^{(2)}, \boldsymbol{\lambda}^{(2)}\right) & \\
-\partial_{\alpha} M \partial_{\alpha}\left(\boldsymbol{\mu}^{(2)}, \boldsymbol{\lambda}^{(2)}\right) .
\end{array}
$$

This relation along with inequalities (10.9) and (10.39) yields the estimate

$$
\left|\partial_{\alpha}\left(\boldsymbol{\mu}^{(1)}, \boldsymbol{\lambda}^{(1)}\right)\right| \leq c|\varepsilon|
$$

The differentiation both sides of equality (9.1) with respect to $\alpha$ leads to the representation

$$
\begin{aligned}
\partial_{\alpha} L_{i j}= & \sigma_{i j}+\int_{\mathbb{T}^{n-1}}\left\{\mathbf{S} \mu^{(i)} \cdot \partial_{\alpha} \boldsymbol{\mu}^{(j)}-\left(\mathbf{J} \boldsymbol{\partial} \boldsymbol{\lambda}^{(i)}+\boldsymbol{\Omega} \boldsymbol{\lambda}^{(i)}\right) \cdot \partial_{\alpha} \boldsymbol{\lambda}^{(j)}\right\} d \boldsymbol{\xi} \\
& +\cdot \int_{\mathbb{T}^{n-1}}\left\{\mathbf{S} \partial_{\alpha} \boldsymbol{\mu}^{(i)} \cdot \boldsymbol{\mu}^{(j)}-\left(\mathbf{J} \boldsymbol{\partial} \boldsymbol{\lambda}^{(j)}+\boldsymbol{\Omega} \boldsymbol{\lambda}^{(j)}\right) \cdot \partial_{\alpha} \boldsymbol{\lambda}^{(i)}\right\} d \boldsymbol{\xi},
\end{aligned}
$$


where

$$
\sigma_{i j}=\int_{\mathbb{T}^{n-1}} \partial_{\alpha} \mathbf{S} \boldsymbol{\mu}^{(i)} \cdot \boldsymbol{\mu}^{(i)} d \boldsymbol{\xi} .
$$

Estimate (8.12) in Theorem 8.2 and estimate (8.31) in Theorem8.4 yield the inequality. that

$$
\left|\sigma_{12}\right|+\left|\sigma_{22}\right| \leq c\left|\boldsymbol{\mu}^{(1)}\right|_{0}\left|\boldsymbol{\mu}^{(2)}\right|_{0}+\left|\boldsymbol{\mu}^{(2)}\right|_{0}^{2} \leq\left. c\left|\varepsilon^{2}, \quad\right| \sigma_{11}|\leq c| \boldsymbol{\mu}^{(1)}\right|_{0} ^{2} \leq c|\varepsilon|\left|\boldsymbol{\mu}_{0}\right|
$$

On the other hand, we have

$$
\begin{aligned}
\mid \int_{\mathbb{T}^{n-1}}\left\{\mathbf{S} \mu^{(i)} \cdot \partial_{\alpha} \boldsymbol{\mu}^{(j)}-\left(\mathbf{J} \boldsymbol{\partial} \boldsymbol{\lambda}^{(i)}+\right.\right. & \left.\left.\boldsymbol{\Omega} \boldsymbol{\lambda}^{(i)}\right) \cdot \partial_{\alpha} \boldsymbol{\lambda}^{(j)}\right\} d \boldsymbol{\xi} \mid \\
& \leq c\left(\left|\boldsymbol{\mu}^{(i)}\right|_{0}\left|\partial_{\alpha} \boldsymbol{\mu}^{(j)}\right|_{0}+\left|\boldsymbol{\lambda}^{(i)}\right|_{1}\left|\partial_{\alpha} \boldsymbol{\lambda}^{(j)}\right|_{0}\right) .
\end{aligned}
$$

Combining this inequality with estimates (10.39)-(10.40) we arrive at the estimate

$$
\begin{aligned}
\left|\int_{\mathbb{T}^{n-1}}\left\{\mathbf{S} \mu^{(i)} \cdot \partial_{\alpha} \boldsymbol{\mu}^{(j)}-\left(\mathbf{J} \boldsymbol{\partial} \boldsymbol{\lambda}^{(i)}+\boldsymbol{\Omega} \boldsymbol{\lambda}^{(i)}\right) \cdot \partial_{\alpha} \boldsymbol{\lambda}^{(j)}\right\} d \boldsymbol{\xi}\right| \\
\quad \leq c|\varepsilon|\left(\left|\boldsymbol{\mu}^{(i)}\right|_{0}+\left|\boldsymbol{\lambda}^{(i)}\right|_{1}\right) \leq c|\varepsilon|
\end{aligned}
$$

which along with (10.42) yields the estimate (10.35) for $\partial_{\alpha} L_{12}$ and $\partial_{\alpha} L_{22}$.

It remains to estimate $\partial_{\alpha} L_{11}$. Since $k=0$, we have

$$
\mathbf{J} \boldsymbol{\partial} \boldsymbol{\lambda}^{(1)}+\boldsymbol{\Omega} \boldsymbol{\lambda}^{(1)}=\mathbf{J} \boldsymbol{\partial} \boldsymbol{\lambda}^{(1)}+\boldsymbol{\lambda}^{(1)} \cdot \mathbf{e}_{2}
$$

Substituting decomposition (8.30) into this relation and using estimate (8.31) we obtain

$$
\left|\mathbf{J} \boldsymbol{\partial} \boldsymbol{\lambda}^{(1)}+\boldsymbol{\Omega} \boldsymbol{\lambda}^{(1)}\right|=\left|\mathbf{J} \boldsymbol{\partial} \boldsymbol{\lambda}_{\varepsilon}+\left(\boldsymbol{\mu}_{0} \cdot \mathbf{t}_{0}\right)+\boldsymbol{\lambda}_{\varepsilon} \cdot \mathbf{e}_{2}\right| \leq c\left|\boldsymbol{\mu}_{0}\right| .
$$

Combining this result with the estimate $\left|\boldsymbol{\mu}^{(1)}\right| \leq c\left|\boldsymbol{\mu}_{0}\right|$ and estimates (10.39) (10.40) we arrive at the inequality

$$
\left|\int_{\mathbb{T}^{n-1}}\left\{\mathbf{S} \mu^{(1)} \cdot \partial_{\alpha} \boldsymbol{\mu}^{(1)}-\left(\mathbf{J} \boldsymbol{\partial} \boldsymbol{\lambda}^{(1)}+\boldsymbol{\Omega} \boldsymbol{\lambda}^{(1)}\right) \cdot \partial_{\alpha} \boldsymbol{\lambda}^{(1)}\right\} d \boldsymbol{\xi}\right| \leq c|\varepsilon|\left|\boldsymbol{\mu}_{0}\right| .
$$

Combing this inequality with estimate (10.42) for $\sigma_{11}$ and identity (10.41) we finally obtain that $\left|\partial_{\alpha} L_{11}\right| \leq c|\varepsilon| \boldsymbol{\mu}_{0} \mid$. This completes the proof of the lemma. 
Let us turn to the proof of Proposition 10.3. Applying Lemma 10.35 we obtain that the quantity $\mathbf{I}_{1}$ given by (10.33) satisfies the inequality

$$
\left|\mathbf{I}_{1}\right| \leq c\left|\partial_{\alpha} L_{11}\right|+c\left|\partial_{\alpha} M\right|\left(\left|\partial_{\alpha} L_{12}\right|+\left|\partial_{\alpha} L_{22}\right|\right) \leq c|\varepsilon|\left|\boldsymbol{\mu}_{0}\right|+c|\varepsilon|\left|\partial_{\alpha} M\right| .
$$

On the other hand, estimates (9.2) and (9.3) in Theorem 9.4 imply the following estimate for the quantity $\boldsymbol{I}_{2}$ given by (10.34)

$$
\left|\mathbf{I}_{2}\right| \leq c\left|L_{12}\right|+c\left|\partial_{\alpha} M\right|\left|L_{22}\right| \leq c|\varepsilon|\left|\boldsymbol{\mu}_{0}\right|+c|\varepsilon|\left|\partial_{\alpha} M\right| \text {. }
$$

Substituting (10.43) and (10.44) into identity (10.32) we arrive at the estimate

$$
\begin{aligned}
& \left|\partial_{\alpha}^{3} \Psi(\alpha, 0)-(2 \pi)^{n-1} \partial_{\alpha} M\right| \\
& \quad \leq c|\varepsilon|\left|\varsigma_{2}\right|\left|\partial_{\alpha} M\right|+c|\varepsilon|\left|\partial_{\alpha} \varsigma_{2}\right||M|+c|\varepsilon|\left(\left|\partial_{\alpha} M\right|+\left|\boldsymbol{\mu}_{0}\right|\right)
\end{aligned}
$$

Recalling estimate (10.13) in Theorem 10.1 for the quantity $\varsigma_{2}$ we finally obtain the desired inequality

$$
\left|\partial_{\alpha}^{3} \Psi(\alpha, 0)-(2 \pi)^{n-1} \partial_{\alpha} M\right| \leq c|\varepsilon|\left(|M|+\left|\partial_{\alpha} M\right|+\left|\boldsymbol{\mu}_{0}\right|\right),
$$

and the proposition follows.

\subsection{Proof of Theorem 2.2}

We are now in a position to prove Theorem 2.2 which is the main result of this work. We begin with the observation that by virtue of Theorem 6.2 the main operator equation (4.16) has an analytic periodic solution $(\varphi, e, m, M)$ for all $(\alpha, k) \in \mathbb{T}^{1} \times[0,1]$ and all $|\varepsilon| \leq \varepsilon_{0}$. This solution define the vector $\boldsymbol{\Theta}(\varphi)=$ $(\mathbf{u}, \mathbf{v}, \mathbf{w}, \mathbf{V}, \mathbf{W}, \boldsymbol{\Lambda}, \mathbf{R})$ such that the corresponding canonical mapping $\boldsymbol{\vartheta}$ put the modified Hamiltonian $H_{m}=H+m z_{1}+2^{-1} M z_{1}^{2}$ into the normal form (2.8). In particular, the modified Hamiltonian has the weakly hyperbolic invariant torus for every $(\alpha, k) \in \mathbb{T}^{1} \times[0,1]$. Recall that $m$ and $M$ are functions of the variables $\alpha$ and $k$. Hence Theorem 2.2 will be proved if we prove that the bifurcation equations

$$
m(\alpha, k)=M(\alpha, k)=0
$$


have a solution $\left(\alpha_{0}, k_{0}\right) \in \mathbb{T}^{1} \times[0,1]$. We claim that such a solution can be defined as a minimizer of the action functional $\Psi(\alpha, k)$ given by (10.6), i.e.,

$$
\Psi\left(\alpha_{0}, k_{0}\right)=\min _{(\alpha, k) \in \mathbb{T}^{1} \times[0,1]} \Psi(\alpha, k) .
$$

The proof of this fact falls into the sequence of lemmas. The first lemma shows that every minimizer $\left(\alpha_{0}, k_{0}\right), k_{0}>0$, of the action functional serves as a solution to the bifurcation equations.

Lemma 10.4. Let all assumptions of Theorem (10.1) be satisfied. Furthermore, assume that

$$
\Psi\left(\alpha_{0}, k_{0}\right)=\min _{(\alpha, k) \in \mathbb{T}^{1} \times[0,1]} \Psi(\alpha, k) \text { and } \Psi\left(\alpha_{0}, 0\right)>\Psi\left(\alpha_{0}, k_{0}\right) .
$$

Then $m\left(\alpha_{0}, k_{0}\right)=M\left(\alpha_{0}, k_{0}\right)=0$.

Proof. Let us prove that $L_{22}\left(\alpha_{0}, k\right)>0$ everywhere on $(0,1)$ but countable discrete set. Notice that the mapping $[0,1] \ni k \rightarrow w_{1}^{*}\left(\alpha_{0}, k\right) \in \mathcal{A}_{\sigma / 2,0}$ is continuously differentiable on $[0,1]$ an is analytic on $(0,1)$. If $w_{1}^{*}$ is not identically equal to zero on $(0,1)$, then there is a countable discrete set $\left\{k_{i}\right\} \subset$ $(0,1)$ such that $\left|w_{1}^{*}\right|_{-1}>0$ outside of this set. It follows from this and estimate (9.2) in Theorem 9.1 that $L_{22}\left(\alpha_{0}, k\right)>0$ for $k \neq k_{i}$.

Let us show that the case when $w_{1}^{*}\left(\alpha_{0}, \cdot\right)$ identically equals zero on $(0,1)$ is impossible. In view of (9.2), the identity $w_{1}^{*}\left(\alpha_{0}, \cdot\right) \equiv 0$ on $(0,1)$ implies the identity $L_{22}\left(\alpha_{0}, \cdot\right) \equiv 0$ on $(0,1)$, and hence implies the identity $\partial_{k} \Psi\left(\alpha_{0}, \cdot\right)=$ $M \partial_{k} M L_{22}\left(\alpha_{0}, \cdot\right) \equiv 0$ on $(0,1)$. The latter is impossible since $\Psi\left(\alpha_{0}, 0\right)>$ $\Psi\left(\alpha_{0}, k_{0}\right)$. Therefore, $L_{22}\left(\alpha_{0}, k\right)>0$ for all $k \neq k_{i}$.

Let us prove that $k_{0}<1$. It follows from inequalities (10.9) and (10.1) that

$$
\left|\partial_{k} M+1\right|+|M+k| \leq c|\varepsilon| .
$$

Hence for all sufficiently small $\varepsilon$ we have

$$
\partial_{k} M \leq-1 / 3, \quad M<-1 / 3 \text { for } k \geq 1 / 2 .
$$

Since $L_{22}\left(\alpha_{0}, k\right)$ is strictly positive a.e. in $(0,1)$, it follows that $\Psi\left(\alpha_{0}, k\right)$ strictly increases on $(1 / 2,1]$. Hence $k_{0}<1$. From this and the conditions of the lemma we conclude that $0<k_{0}<1$.

Let us prove that $M\left(\alpha_{0}, k_{0}\right)=0$. Notice that for all sufficiently small $|\varepsilon|$, we have $\partial_{k} M \geq-1+c|\varepsilon|<0$. Hence $\partial_{k} M L_{22}\left(\alpha_{0}, k\right)<0$ almost everywhere on $(0,1)$. Next, identity (10.11) in Theorem 10.1 implies $\partial_{k} \Psi\left(\alpha_{0}, k\right)=$ 
$M \partial_{k} M L_{22}\left(\alpha_{0}, k\right)$. If $M\left(\alpha_{0}, k_{0}\right) \neq 0$, then the function $\Psi\left(\alpha_{0}, k\right)$ is strictly monotone in a neighborhood of $k_{0}$. This contradicts to the assumption that $\left(\alpha_{0}, k_{0}\right)$ is a minimizer of $\Psi$. Hence $M\left(\alpha_{0}, k_{0}\right)$ equals zero.

It remains to prove that $m\left(\alpha_{0}, k_{0}\right)=0$. To this end, notice that identity (10.10) in Theorem 10.1 along with the equality $M\left(\alpha_{0}, k_{0}\right)=0$ yields

$$
0=\partial_{\alpha} \Psi\left(\alpha_{0}, k_{0}\right)=(2 \pi)^{n-1}(m+\alpha M)+\varsigma_{1} M=(2 \pi)^{n-1} m\left(\alpha_{0}, k_{0}\right),
$$

which completes the proof.

Lemma 10.5. Let all assumptions of Theorem (10.1) be satisfied. Furthermore, assume that

$$
\Psi\left(\alpha_{0}, 0\right)=\min _{(\alpha, k) \in \mathbb{T}^{1} \times[0,1]} \Psi(\alpha, k) \text { and } \mathbf{K}_{0} \boldsymbol{\mu}_{0} \cdot \boldsymbol{\mu}_{0}<0 .
$$

Then $m\left(\alpha_{0}, 0\right)=M\left(\alpha_{0}, 0\right)=0$.

Proof. We split the proof into two steps.

Step 1. First we prove the lemma under the additions assumptions that

$$
\left\|w_{1}^{*}\left(\alpha_{0}, k\right)\right\|_{\sigma / 2,0}>0 \text { for a.e. } k \in(0,1) .
$$

Let us prove that $M\left(\alpha_{0}, 0\right)=0$. Suppose, contrary to our claim, that $M\left(\alpha_{0}, 0\right) \neq 0$. Notice that $\partial_{k} M \leq-1+c|\varepsilon|<0$. On the other hand, estimate (9.2) in Theorem 9.1 yields $L_{22} \geq c^{-1}\left|w_{1}^{*}\right|_{-1}$. Hence $\partial_{k} M L_{22}\left(\alpha_{0}, k\right)<0$ almost everywhere on $(0,1)$. If $M\left(\alpha_{0}, 0\right)>0$, then $\Psi\left(\alpha_{0}, k\right)$ strictly decreases in a neighborhood of $k=0$. This contradicts to the assumptions that $\left(\alpha_{0}, 0\right)$ is a minimizer of $\Psi$. Hence $M\left(\alpha_{0}, 0\right) \leq 0$. Next, representation (10.12) in Theorem 10.1 yields the inequality

$$
\begin{aligned}
0 \leq \partial_{\alpha}^{2} \Psi\left(\alpha_{0}, 0\right)= & (2 \pi)^{n-1} M\left(\alpha_{0}, 0\right)+\varsigma_{2} M\left(\alpha_{0}, 0\right) \\
& +L_{11}\left(\alpha_{0}, 0\right)+2 L_{12} \partial_{\alpha} M\left(\alpha_{0}, 0\right)+L_{22} \partial_{\alpha} M\left(\alpha_{0}, 0\right)^{2}
\end{aligned}
$$

In view of Theorem 10.1, we have $\left|\varsigma_{2}\right| \leq c|\varepsilon|$. Hence the inequality

$$
\left|M\left(\alpha_{0}, 0\right)\right|=-M\left(\alpha_{0}, 0\right) \leq L_{11}\left(\alpha_{0}, 0\right)+2 L_{12} \partial_{\alpha} M\left(\alpha_{0}, 0\right)+L_{22} \partial_{\alpha} M\left(\alpha_{0}, 0\right)^{2}
$$

holds true for all sufficiently small $\varepsilon$. Next, estimate (9.3) in Theorem (9.1) implies the inequality

$$
\begin{aligned}
L_{11}+2\left|\partial_{\alpha} M\right|\left|L_{12}\right| \leq(2 \pi)^{n-1} \mathbf{K}_{0} \boldsymbol{\mu}_{0} \cdot \boldsymbol{\mu}_{0}+c|\varepsilon|\left|\boldsymbol{\mu}_{0}\right|^{2}+c|\varepsilon|\left|\boldsymbol{\mu}_{0}\right|\left|\partial_{\alpha} M\right| \\
\leq(2 \pi)^{n-1} \mathbf{K}_{0} \boldsymbol{\mu}_{0} \cdot \boldsymbol{\mu}_{0}+c|\varepsilon|\left|\boldsymbol{\mu}_{0}\right|^{2}+c\left|\varepsilon \| \partial_{\alpha} M\right|^{2} .
\end{aligned}
$$


Recall that

$$
-c^{-1}\left|\boldsymbol{\mu}_{0}\right|^{2} \leq\left|K_{0} \boldsymbol{\mu}_{0} \cdot \boldsymbol{\mu}_{0}\right| \leq-c\left|\boldsymbol{\mu}_{0}\right|^{2}
$$

for some positive constant $c$ independent of $\varepsilon$. From this we conclude that

$$
L_{11}+2\left|\partial_{\alpha} M\right|\left|L_{12}\right| \leq \mathbf{K}_{0} \boldsymbol{\mu}_{0} \cdot \boldsymbol{\mu}_{0}+c|\varepsilon|\left|\partial_{\alpha} M\right|^{2}
$$

for all sufficiently small $\varepsilon$. Substituting this inequality into (10.51) and noting that $L_{22} \leq c \varepsilon^{2}$ we conclude that

$$
\left|M\left(\alpha_{0}, 0\right)\right|-\mathbf{K}_{0} \boldsymbol{\mu}_{0} \cdot \boldsymbol{\mu}_{0} \leq c|\varepsilon|\left|\partial_{\alpha} M\right|^{2},
$$

which gives

$$
\left|M\left(\alpha_{0}, 0\right)\right|+\left|\boldsymbol{\mu}_{0}\right|^{2} \leq c|\varepsilon|\left|\partial_{\alpha} M\right|^{2},
$$

It follows from representation (10.50) and estimates (9.2), (9.3) in Theorem 9.1 that

$$
\begin{aligned}
0 \leq \partial_{\alpha}^{2} \Psi\left(\alpha_{0}, 0\right) \leq c\left|M\left(\alpha_{0}, 0\right)\right| & +\left|L_{11}\left(\alpha_{0}, 0\right)\right|+ \\
2\left|L_{12}\right|\left|\partial_{\alpha} M\left(\alpha_{0}, 0\right)\right|+\left|L_{22}\right| & \partial_{\alpha} M\left(\alpha_{0}, 0\right)^{2} \leq c\left|M\left(\alpha_{0}, 0\right)\right|+c\left|\boldsymbol{\mu}_{0}\right|^{2} \\
& +c|\varepsilon|\left|\boldsymbol{\mu}_{0}\right|\left|\partial_{\alpha} M\left(\alpha_{0}, 0\right)\right|+c|\varepsilon|\left|\partial_{\alpha} M\left(\alpha_{0}, 0\right)\right|^{2}
\end{aligned}
$$

Substituting estimate (10.53) into the right hand side of this inequality we obtain

$$
0 \leq \partial_{\alpha}^{2} \Psi\left(\alpha_{0}, 0\right) \leq c|\varepsilon|\left|\partial_{\alpha} M\left(\alpha_{0}, 0\right)\right|^{2} .
$$

Let us estimate the third derivatives of $\Psi$. Combining inequality (10.31) in Proposition 10.2 and inequality (10.53) we obtain

$$
\left|\partial_{\alpha}^{3} \Psi\left(\alpha_{0}, 0\right)-(2 \pi)^{n-1} \partial_{\alpha} M\left(\alpha_{0}, 0\right)\right| \leq c|\varepsilon|\left|\partial_{\alpha} M\left(\alpha_{0}, 0\right)\right| .
$$

It follows that the inequality

$$
\left|\partial_{\alpha}^{3} \Psi\left(\alpha_{0}, 0\right)\right| \geq\left|\partial_{\alpha} M\left(\alpha_{0}, 0\right)\right|
$$

holds for all small $\varepsilon$. Since $\Psi_{0}\left(\alpha_{0}, 0\right)$ is analytic and takes the minimum at point $\alpha_{0}$ we have the Taylor expansion

$$
0 \leq \Psi\left(\alpha_{0}+t, 0\right)-\Psi\left(\alpha_{0}, 0\right)=A t^{2}+B t^{3}+C t^{4},
$$

where

$$
A=2^{-1} \partial_{\alpha}^{2} \Psi\left(\alpha_{0}, 0\right), \quad B=6^{-1} \partial_{\alpha}^{2} \Psi\left(\alpha_{0}, 0\right), \quad,|C| \leq c_{4},
$$


where $c_{4}$ is independent of $\varepsilon$. Notice that this relation holds true for all $t \in \mathbb{R}^{1}$. Estimates (10.54) and (10.55) imply the inequalities

$$
|A| \leq c_{2}|\varepsilon|\left|\partial_{\alpha} M\right|^{2}, \quad|B| \geq 6^{-1}\left|\partial_{\alpha} M\right|
$$

Now set $t=-\delta B$, where $\delta$ are an arbitrary positive number. We have

$$
\begin{aligned}
0 \leq \Psi\left(\alpha_{0}+t, 0\right)-\Psi\left(\alpha_{0}, 0\right)=A t^{2}+B t^{3}+C t^{4}= \\
B^{4} \delta^{3}\left(A B^{-2} \delta^{-1}-1+\delta C\right)
\end{aligned}
$$

Notice that $\left|A B^{-2}\right| \leq c|\varepsilon|$. Obviously, the right hand side of (10.57) is negative for $\delta=|\varepsilon|^{1 / 2}$ and small $\varepsilon$. This contradiction proves the equality $M\left(\alpha_{0}, 0\right)=0$. It remains to note that the equality $m\left(\alpha_{0}, k\right)=0$ obviously follows from the relations

$$
0=\partial_{\alpha} \Psi\left(\alpha_{0}, 0\right)=(2 \pi)^{n-1}(m+\alpha M)+\varsigma_{1} M=(2 \pi)^{n-1} m\left(\alpha_{0}, 0\right) .
$$

Step 2. It remains to consider the degenerate case when $w_{1}^{*}\left(\alpha_{0}, k\right)=0$ for all $k \in[0,1]$. In view of estimate (8.12) we have in this case

$$
\boldsymbol{\lambda}^{(2)}\left(\alpha_{0}, k\right)=0, \quad \boldsymbol{\mu}^{(2)}\left(\alpha_{0}, k\right)=0 \text { for all } k \in[0,1] .
$$

Recalling representation (9.1) for $L_{i j}$ we conclude that

$$
L_{12}\left(\alpha_{0}, k\right)=0, \quad L_{22}\left(\alpha_{0}, k\right)=0 \text { for all } k \in[0,1] .
$$

It follows from this and formula (10.11) that $\partial_{k} \Psi\left(\alpha_{0}, k\right)=0$ and hence

$$
\Psi\left(\alpha_{0}, k\right)=\min _{(\alpha, l) \in \mathbb{T}^{1} \times[0,1]} \Psi(\alpha, l) \text { for all } k \in[0,1] .
$$

In other words, $\Psi$, the whole segment $\left\{\alpha_{0}\right\} \times[0,1]$ consists of the minimizers of $\Psi$.

Let us prove that there is $k_{0} \in[0,1]$ such that $M\left(\alpha_{0}, k_{0}\right)=0$. We begin with the observation that $\left|M\left(\alpha_{0}, 1\right)+1\right| \leq c|\varepsilon|$. Hence, $M\left(\alpha_{0}, 1\right)<0$ for all sufficiently small $\varepsilon$. Therefore, it suffices to prove that $M\left(\alpha_{0}, 0\right) \geq 0$. Suppose, contrary to our claim, that $M\left(\alpha_{0}, 0\right)<0$ Since $\left(\alpha_{0}, 0\right)$ is a minimizer of $\Psi$ and $L_{12}=L_{22}=0$ for $\alpha=\alpha_{0}$, representation (10.12) in Theorem 10.1 implies the inequality

$$
0 \leq \partial_{\alpha}^{2} \Psi\left(\alpha_{0}, 0\right)=(2 \pi)^{n-1} M\left(\alpha_{0}, 0\right)+\varsigma_{2} M\left(\alpha_{0}, 0\right)+L_{11}\left(\alpha_{0}, 0\right) .
$$


It follows from this, estimate (9.3) in Theorem 9.1, and the inequality $\left|\varsigma_{2}\right| \leq$ $c|\varepsilon|$ that

$$
\begin{aligned}
0 \leq \partial_{\alpha}^{2} \Psi\left(\alpha_{0}, 0\right)=(2 \pi)^{n-1} M\left(\alpha_{0}, 0\right)+ \\
\mathbf{K}_{0} \boldsymbol{\mu}_{0} \cdot \boldsymbol{\mu}_{0}+c|\varepsilon|\left|M\left(\alpha_{0}, 0\right)\right|+c|\varepsilon|\left|\boldsymbol{\mu}_{0}\right|^{2} .
\end{aligned}
$$

Recall that $M\left(\alpha_{0}, 0\right)<0$ and $\mathbf{K}_{0} \boldsymbol{\mu}_{0} \cdot \boldsymbol{\mu}_{0} \leq-c\left|\boldsymbol{\mu}_{0}\right|^{2}$. It follows from this that the right hand side of (10.60) is negative for all sufficiently small $\varepsilon$. The contradiction prove the inequality $M\left(\alpha_{0}, 0\right) \geq 0$. Since $M\left(\alpha_{0}, 1\right)$ is negative, there is $k_{0}$ such that $M\left(\alpha_{0}, k_{0}\right)=0$. On the other hand, $\Psi$ takes the minimum at the point $\left(\alpha_{0}, k_{0}\right)$. It follows from this that $\partial_{\alpha} \Psi\left(\alpha_{0}, k_{0}\right)=$ $(2 \pi)^{n-1} m\left(\alpha_{0}, k_{0}\right)=0$. This completes the proof of the lemma.

It remains to note that the statement of Theorem 2.2 is a straightforward consequence of Lemmas 10.4 and 10.5.

\section{A Proof of Theorem 3.1}

Let $\vartheta$ is defined by (3.1). It follows that

$$
\boldsymbol{\vartheta}^{\prime}=\left(\begin{array}{ccc}
A_{11} & 0 & 0 \\
A_{21} & A_{22} & A_{23} \\
A_{31} & 0 & A_{33}
\end{array}\right), \quad \boldsymbol{\vartheta}^{\prime \top}=\left(\begin{array}{ccc}
A_{11}^{\top} & A_{21}^{\top} & A_{31}^{\top} \\
0 & A_{22}^{\top} & 0 \\
0 & A_{23}^{\top} & A_{33}^{\top}
\end{array}\right),
$$

where

$$
\begin{gathered}
A_{11}=\mathbf{I}+\mathbf{u}^{\prime}, \quad A_{22}=\mathbf{V}, \quad A_{33}=\mathbf{W}, \\
A_{21}=\mathbf{v}_{\xi}^{\prime}+(\mathbf{V} \boldsymbol{\eta})_{\xi}^{\prime}+(\boldsymbol{\Lambda} \boldsymbol{\zeta})_{\xi}^{\prime}+\frac{1}{2}\left(\boldsymbol{\zeta}^{\top} \mathbf{R} \boldsymbol{\zeta}\right)_{\xi}^{\prime}, \\
A_{31}=\mathbf{w}^{\prime}+(\mathbf{W} \boldsymbol{\zeta})_{\xi}^{\prime}, \quad A_{23}=\boldsymbol{\Lambda}+\mathbf{R} \boldsymbol{\zeta} .
\end{gathered}
$$

Here notation $\mathbf{R} \boldsymbol{\zeta}$ stands for $(n-1) \times 2$ matrix with the entries

$$
\{\mathbf{R} \zeta\}_{i p}=\left\{\mathbf{R}_{i}\right\}_{p q} \zeta_{q}, \quad 1 \leq i \leq n-1, \quad p=1,2 .
$$

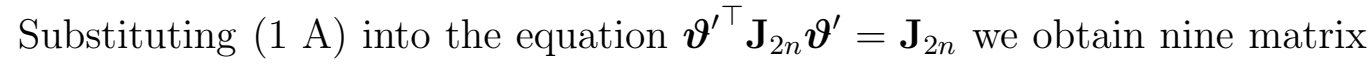
equations. Four of those are nontrivial:

$$
\begin{gathered}
A_{11}^{\top} A_{22}=\mathbf{I}, \quad A_{33}^{\top} \mathbf{J} A_{33}=\mathbf{J}, \\
A_{11}^{\top} A_{23}+A_{31}^{\top} \mathbf{J} A_{33}=0, \\
A_{11}^{\top} A_{21}-A_{21}^{\top} A_{11}+A_{31}^{\top} \mathbf{J} A_{31}=0 .
\end{gathered}
$$


The remaining five equations are either trivial or can be obtained from $(4 \mathrm{~A})-(6 \mathrm{~A})$ by transposition. Equations (4A) along with (2A) give

$$
\begin{array}{r}
\mathbf{V}=\left(\mathbf{I}_{n-1}+\mathbf{u}^{\prime}\right)^{-\top}, \\
\mathbf{W}^{\top} \mathbf{J W}=\mathbf{J} \text { or equivalently } \operatorname{det} \mathbf{W}=1 .
\end{array}
$$

In view of $(2 \mathrm{~A})$ we can rewrite equation $(5 \mathrm{~A})$ in the form

$$
\boldsymbol{\Lambda}+\mathbf{R} \boldsymbol{\zeta}=-\boldsymbol{V}\left(\mathbf{w}_{\xi}^{\prime}+(\mathbf{W} \boldsymbol{\zeta})_{\xi}^{\prime}\right)^{\top} \mathbf{J W} \text { for all } \boldsymbol{\zeta} \in \mathbb{R}^{2} .
$$

It follows that

$$
\boldsymbol{\Lambda}=-\boldsymbol{V}\left(\mathbf{w}_{\xi}^{\prime}\right)^{\top} \mathbf{J W}
$$

and

$$
\mathbf{R} \boldsymbol{\zeta}=-\boldsymbol{V}(\mathbf{W} \boldsymbol{\zeta})_{\xi}^{\prime \top} \mathbf{J W} \text { for all } \boldsymbol{\zeta} \in \mathbb{R}^{2} .
$$

Recalling the identities

$$
\{\mathbf{R} \boldsymbol{\zeta}\}_{i j}=R_{i, j q} \zeta_{q}, \quad\left\{(\mathbf{W} \boldsymbol{\zeta})_{\xi}^{\prime \top}\right\}_{k p}=\frac{\partial}{\partial \xi_{k}}\left(W_{p q} \zeta_{q}\right)
$$

we obtain

$$
R_{i, q j} \zeta_{q}=-V_{i k} \frac{\partial}{\partial \xi_{k}}\left(W_{p q} \zeta_{q}\right)\{\mathbf{J W}\}_{p j} \text { for all } \boldsymbol{\zeta} \in \mathbb{R}^{2} .
$$

Since the matrix $\mathbf{R}_{i}$ is symmetric, we obtain

$$
\mathbf{R}_{i}=-V_{i k} \frac{\partial}{\partial \xi_{k}}\left(\mathbf{W}^{\top}\right) \mathbf{J} \mathbf{W} .
$$

Let us turn to equation (6A) . It is equivalent to the system of four matrix equations

$$
\begin{gathered}
\frac{1}{2} \mathbf{V}^{-1}\left(\boldsymbol{\zeta}^{\top} \mathbf{R} \boldsymbol{\zeta}\right)_{\xi}^{\prime}-\frac{1}{2}\left(\boldsymbol{\zeta}^{\top} \mathbf{R} \boldsymbol{\zeta}\right)_{\xi}^{\prime \top} \mathbf{V}^{-\top}+(\mathbf{W} \boldsymbol{\zeta})_{\xi}^{\prime \top} \mathbf{J}(\mathbf{W} \boldsymbol{\zeta})_{\xi}^{\prime}=0, \\
\mathbf{V}^{-1}(\boldsymbol{\Lambda} \boldsymbol{\zeta})_{\xi}^{\prime}-(\boldsymbol{\Lambda} \boldsymbol{\zeta})_{\xi}^{\prime \top} \mathbf{V}^{-\top}+(\mathbf{w})_{\xi}^{\prime \top} \mathbf{J}(\mathbf{W} \boldsymbol{\zeta})_{\xi}^{\prime}+(\mathbf{W} \boldsymbol{\zeta})_{\xi}^{\prime \top} \mathbf{J}(\mathbf{w})_{\xi}^{\prime}=0 \\
\mathbf{V}^{-1}(\mathbf{V} \boldsymbol{\eta})_{\xi}^{\prime}-(\mathbf{V} \boldsymbol{\eta})_{\xi}^{\prime \top} \mathbf{V}^{-\top}=0 \\
\mathbf{V}^{-1}(\mathbf{v})_{\xi}^{\prime}-(\mathbf{v})_{\xi}^{\prime \top} \mathbf{V}^{-\top}+(\mathbf{w})_{\xi}^{\prime \top} \mathbf{J}(\mathbf{w})_{\xi}^{\prime}=0,
\end{gathered}
$$


which hold true for all $\zeta \in \mathbb{R}^{2}$ and for all $\boldsymbol{\eta} \in \mathbb{R}^{n-1}$. Let us proof that equations $(10 \mathrm{~A})-(12 \mathrm{~A})$ are consequence of $(7 \mathrm{~A})-(9 \mathrm{~A})$. We start with equation $(10 \mathrm{~A})$. It follows from $(7 \mathrm{~A})$ that

$$
\mathbf{x}=\boldsymbol{\xi}+\mathbf{u}(\boldsymbol{\xi}), \quad \mathbf{V}=\left(\mathbf{I}+\mathbf{u}^{\prime}\right)^{-\top} \equiv\left(\mathbf{x}_{\xi}^{\prime}\right)^{-\top}=\left(\boldsymbol{\xi}_{x}^{\prime}\right)^{\top},
$$

and hence

$$
\frac{\partial}{\partial x_{i}}=V_{i k} \frac{\partial}{\partial \xi_{k}}, \quad \nabla_{x}=\mathbf{V} \nabla_{\xi} .
$$

Multiplying both sides of equation $(10 \mathrm{~A})$ by $\mathbf{V}^{\top}$ from the right and by $\mathbf{V}$ from the left we can rewrite this equation in the equivalent form

$$
\frac{1}{2}\left(\boldsymbol{\zeta}^{\top} \mathbf{R} \boldsymbol{\zeta}\right)_{x}^{\prime}-\frac{1}{2}\left(\boldsymbol{\zeta}^{\top} \mathbf{R} \boldsymbol{\zeta}\right)_{x}^{\prime \top}+(\mathbf{W} \boldsymbol{\zeta})_{x}^{\prime \top} \mathbf{J}(\mathbf{W} \boldsymbol{\zeta})_{x}^{\prime}=0
$$

In view of $(9 \mathrm{~A})$, we have

$$
\boldsymbol{\zeta}^{\top} \mathbf{R}_{i} \boldsymbol{\zeta}=-\boldsymbol{\zeta}^{\top} V_{i k} \frac{\partial}{\partial \xi_{k}}\left(\mathbf{W}^{\top}\right) \mathbf{J W} \boldsymbol{\zeta}=-\boldsymbol{\zeta}^{\top} \frac{\partial}{\partial x_{i}}\left(\mathbf{W}^{\top}\right) \mathbf{J W} \boldsymbol{\zeta},
$$

which implies

$$
\left\{\left(\boldsymbol{\zeta}^{\top} \mathbf{R}_{i} \boldsymbol{\zeta}\right)_{x}^{\prime}\right\}_{i j}=-\boldsymbol{\zeta}^{\top} \frac{\partial}{\partial x_{j}}\left(\frac{\partial \mathbf{W}^{\top}}{\partial x_{i}} \mathbf{J W}\right) \boldsymbol{\zeta}
$$

Thus we get

$$
\begin{gathered}
\frac{1}{2}\left\{\left(\boldsymbol{\zeta}^{\top} \mathbf{R} \boldsymbol{\zeta}\right)_{x}^{\prime}-\left(\boldsymbol{\zeta}^{\top} \mathbf{R} \boldsymbol{\zeta}_{x}^{\prime}{ }_{x}^{\top}\right\}_{i j}=-\frac{1}{2} \boldsymbol{\zeta}^{\top} \frac{\partial}{\partial x_{j}}\left(\frac{\partial \mathbf{W}^{\top}}{\partial x_{i}} \mathbf{J W}\right) \boldsymbol{\zeta}+\right. \\
\frac{1}{2} \boldsymbol{\zeta}^{\top} \frac{\partial}{\partial x_{i}}\left(\frac{\partial \mathbf{W}^{\top}}{\partial x_{j}} \mathbf{J W}\right) \boldsymbol{\zeta}=\frac{1}{2} \boldsymbol{\zeta}^{\top}\left(\frac{\partial \mathbf{W}^{\top}}{\partial x_{j}} \mathbf{J} \frac{\partial \mathbf{W}}{\partial x_{i}}-\frac{\partial \mathbf{W}^{\top}}{\partial x_{i}} \mathbf{J} \frac{\partial \mathbf{W}}{\partial x_{j}}\right) \boldsymbol{\zeta} .
\end{gathered}
$$

On the other hand, we have

$$
\left\{(\mathbf{W} \boldsymbol{\zeta})_{x}^{\prime \top} \mathbf{J}(\mathbf{W} \boldsymbol{\zeta})_{x}^{\prime}\right\}_{i j}=\boldsymbol{\zeta}^{\top}\left(\frac{\partial \mathbf{W}^{\top}}{\partial x_{i}} \mathbf{J} \frac{\partial \mathbf{W}}{\partial x_{j}}\right) \boldsymbol{\zeta} .
$$

Noting that $\boldsymbol{\zeta}^{\top} \mathbf{A} \boldsymbol{\zeta}=\boldsymbol{\zeta}^{\top} \mathbf{A}^{\top} \boldsymbol{\zeta}$ for every matrix $\mathbf{A}$ and setting

$$
\mathbf{A}=\frac{\partial \mathbf{W}^{\top}}{\partial x_{i}} \mathbf{J} \frac{\partial \mathbf{W}}{\partial x_{j}}, \quad \mathbf{A}^{\top}=-\frac{\partial \mathbf{W}^{\top}}{\partial x_{j}} \mathbf{J} \frac{\partial \mathbf{W}}{\partial x_{i}},
$$


we arrive at the identity

$$
\left\{(\mathbf{W} \boldsymbol{\zeta})_{x}^{\prime \top} \mathbf{J}(\mathbf{W} \boldsymbol{\zeta})_{x}^{\prime}\right\}_{i j}=\frac{1}{2} \boldsymbol{\zeta}^{\top}\left(\frac{\partial \mathbf{W}^{\top}}{\partial x_{i}} \mathbf{J} \frac{\partial \mathbf{W}}{\partial x_{j}}-\frac{\partial \mathbf{W}^{\top}}{\partial x_{j}} \mathbf{J} \frac{\partial \mathbf{W}}{\partial x_{i}}\right) \boldsymbol{\zeta} .
$$

Combining (16 A ) and (17 A ) gives (15A). Hence (7A) and (9A) imply $\left(\begin{array}{ll}10 & \mathrm{~A}\end{array}\right)$. Let us turn to equation (11 A $)$. Arguing as before we can rewrite it in the equivalent form

$$
(\boldsymbol{\Lambda} \boldsymbol{\zeta})_{x}^{\prime}-(\boldsymbol{\Lambda} \boldsymbol{\zeta})_{x}^{\prime \top}+(\mathbf{w})_{x}^{\prime \top} \mathbf{J}(\mathbf{W} \boldsymbol{\zeta})_{x}^{\prime}+(\mathbf{W} \boldsymbol{\zeta})_{x}^{\prime \top} \mathbf{J}(\mathbf{w})_{x}^{\prime}=0
$$

Next, equality (8 A $)$ yields

$$
\Lambda=-\mathbf{V} \nabla_{\xi} \mathbf{w} \mathbf{J W}=-\nabla_{x} \mathbf{w} \mathbf{J W}
$$

which leads to

$$
\left\{(\boldsymbol{\Lambda} \boldsymbol{\zeta})_{x}^{\prime}\right\}_{i j}=-\frac{\partial}{\partial x_{j}}\left(\frac{\partial \mathbf{w}^{\top}}{\partial x_{i}}(\mathbf{J} \mathbf{W}) \boldsymbol{\zeta}\right)
$$

and hence

$$
\left.\left.\left\{(\boldsymbol{\Lambda} \boldsymbol{\zeta})_{x}^{\prime}-(\boldsymbol{\Lambda} \boldsymbol{\zeta})_{x}^{\prime \top}\right\}_{i j}=\frac{\partial \mathbf{w}^{\top}}{\partial x_{j}} \mathbf{J} \frac{\partial}{\partial x_{i}}(\mathbf{W} \boldsymbol{\zeta})\right)-\frac{\partial \mathbf{w}^{\top}}{\partial x_{i}} \mathbf{J} \frac{\partial}{\partial x_{j}}(\mathbf{W} \boldsymbol{\zeta})\right) .
$$

This relations can be rewritten in the matrix form

$$
(\boldsymbol{\Lambda} \boldsymbol{\zeta})_{x}^{\prime}-(\boldsymbol{\Lambda} \boldsymbol{\zeta})_{x}^{\prime \top}=-\left(\mathbf{w}_{x}^{\prime}\right)^{\top} \mathbf{J}(\mathbf{W} \boldsymbol{\zeta})_{x}^{\prime}+\left(\left(\mathbf{w}_{x}^{\prime}\right)^{\top} \mathbf{J}(\mathbf{W} \boldsymbol{\zeta})_{x}^{\prime}\right)^{\top} .
$$

Notice that

$$
\left(\left(\mathbf{w}_{x}^{\prime}\right)^{\top} J(\mathbf{W} \boldsymbol{\zeta})_{x}^{\prime}\right)^{\top}=(\mathbf{W} \boldsymbol{\zeta})_{x}^{\prime \top} \mathbf{J}^{\top} w_{x}^{\prime}=-(\mathbf{W} \boldsymbol{\zeta})_{x}^{\prime \top} \mathbf{J}^{\top} w_{x}^{\prime}
$$

Combining this result with $(19 \mathrm{~A})$ we arrive at equation $(18 \mathrm{~A})$. Since the latter is equivalent to (11A $)$, we obtain that $(\underline{11 A})$ follows from (17A $)$ $(\underline{8 \mathrm{~A}})$. Now consider the equation (12A) . In view of identities $(\underline{14 \mathrm{~A}})$ it can be rewritten in the equivalent form

$$
(\mathbf{V} \boldsymbol{\eta})_{\xi}^{\prime} \mathbf{V}^{\top}-\mathbf{V}(\mathbf{V} \boldsymbol{\eta})_{\xi}^{\prime \top} \equiv(\mathbf{V} \boldsymbol{\eta})_{x}^{\prime}-(\mathbf{V} \boldsymbol{\eta})_{x}^{\prime \top}=0
$$

On the other hand, formula $(7 \mathrm{~A})$ yields

$$
\{\mathbf{V} \boldsymbol{\eta}\}_{i}=\frac{\partial \boldsymbol{\xi}^{\top}}{\partial x_{i}} \cdot \boldsymbol{\eta}
$$


Hence

$$
\left\{(\mathbf{V} \boldsymbol{\eta})_{x}^{\prime}\right\}_{i j}=\frac{\partial^{2} \boldsymbol{\xi}^{\top}}{\partial x_{i} \partial x_{j}} \cdot \boldsymbol{\eta}
$$

Hence the matrix $(\mathbf{V} \boldsymbol{\eta})_{x}^{\prime}$ is symmetric, and (20 A) is a straightforward consequence of $(7 \mathrm{~A})$. Thus we show that equations $(10 \mathrm{~A})-(12 \mathrm{~A})$ directly follows from ( $(7 \mathrm{~A})-(9 \mathrm{~A})$. Let us consider equation $(13 \mathrm{~A})$. We prove that it is not trivial and leads to the desired representation $\mathbf{v}$. It follows from (7A) that equation (13 $\mathrm{A}$ ) can be written in the form

$\frac{\partial}{\partial \xi_{i}}\left(\xi_{k}+u_{k}\right) \frac{\partial v_{k}}{\partial \xi_{j}}-\frac{\partial}{\partial \xi_{j}}\left(\xi_{k}+u_{k}\right) \frac{\partial v_{k}}{\partial \xi_{i}}+\frac{\partial w_{1}}{\partial \xi_{i}} \frac{\partial w_{2}}{\partial \xi_{j}}-\frac{\partial w_{1}}{\partial \xi_{j}} \frac{\partial w_{2}}{\partial \xi_{i}}=0, \quad 1 \leq i, j \leq n-1$,

which is equivalent to

$$
d\left(\xi_{k}+u_{k}\right) \wedge d v_{k}+d w_{1} \wedge w_{2}=0 .
$$

Next, multiplying (13 A) by $\mathbf{V}$ from the left and by $\mathbf{V}^{\top}$ from the right we obtain

$$
\mathbf{v}_{\xi}^{\prime} \mathbf{V}^{\top}-\mathbf{V} \mathbf{v}_{\xi}^{\prime \top}+\mathbf{V}\left(\mathbf{w}_{\xi}^{\prime}\right)^{\top} \mathbf{J} \mathbf{w}_{\xi}^{\prime} \mathbf{V}^{\top} \equiv \mathbf{v}_{x}-\mathbf{v}_{x}^{\prime \top}+\left(\mathbf{w}_{x}^{\prime}\right)^{\top} \mathbf{J} \mathbf{w}_{x}^{\prime}=0
$$

This means that

$$
\begin{array}{r}
\frac{\partial v_{i}}{\partial x_{j}}-\frac{\partial v_{j}}{\partial x_{i}}+\frac{\partial w_{1}}{\partial x_{i}} \frac{\partial w_{2}}{\partial x_{j}}-\frac{\partial w_{1}}{\partial x_{j}} \frac{\partial w_{2}}{\partial x_{i}}= \\
\frac{\partial}{\partial x_{j}}\left(v_{i}+w_{2} \frac{\partial w_{1}}{\partial x_{i}}\right)-\frac{\partial}{\partial x_{i}}\left(v_{j}+w_{2} \frac{\partial w_{1}}{\partial x_{j}}\right)=0
\end{array}
$$

Since $\mathbf{v}$ and $\mathbf{w}$ are analytic an $2 \pi$-periodic, it follows that there exist analytic $2 \pi$-periodic function $\varphi_{0}$ with zero mean value and a constant $\boldsymbol{\beta} \in \mathbb{R}^{n-1}$ such that

$$
\beta_{i}+\frac{\partial \varphi_{0}}{\partial x_{i}}=v_{i}+w_{2} \frac{\partial w_{1}}{\partial x_{i}}
$$

this leads to the desired representation for $\mathbf{v}$

$$
\mathbf{v}=\beta+\nabla_{x} \varphi_{0}-w_{2} \nabla_{x} w_{2}=\beta+\mathbf{V}\left(\nabla_{\xi} \varphi_{0}-w_{2} \nabla_{\xi} w_{2}\right)
$$

Formulae $(\underline{\mathrm{A}} \mathrm{A})-(\underline{\mathrm{A}})$ and $(21 \mathrm{~A})$ give general solution to equation $\boldsymbol{\vartheta}^{\prime \top} \mathbf{J}_{2 n} \boldsymbol{\vartheta}^{\prime}=$ $\mathbf{J}_{2 n}$ and completely determine the totality of canonical mappings $\boldsymbol{\vartheta}$. 


\section{B Proof of Theorem 3.2}

\section{B.1 Proof of $(\mathbf{i})$.}

Choose an arbitrary analytic

$$
\boldsymbol{\varphi}=\left(\beta, \varphi_{0}, \mathbf{u}, \mathbf{w}, W_{11}, W_{12}, W_{21}\right)
$$

and consider the vector field

$$
\boldsymbol{\Theta}(\boldsymbol{\varphi})=\left(\mathbf{u}, \mathbf{v}, \mathbf{w}, \mathbf{V}, \boldsymbol{\Lambda}, \mathbf{W}, \mathbf{R}_{i}\right)
$$

defined by (3.8). Next, choose an arbitrary

$$
\boldsymbol{\Upsilon}=\left(\boldsymbol{\nu}, \psi_{0}, \boldsymbol{\chi}, \boldsymbol{\lambda}, \Gamma_{11}, \Gamma_{12}, \Gamma_{21}\right) \in X_{\sigma, d-1}(r),
$$

and set $\boldsymbol{\mu}=\boldsymbol{\nu}+\nabla \psi_{0}, \Gamma_{22}=-\Gamma_{11}$. Our task is to find the vector field

$$
\delta \boldsymbol{\varphi}=\left(\delta \beta, \delta \varphi_{0}, \delta \mathbf{u}, \delta \mathbf{w}, \delta W_{11}, \delta W_{12}, \delta W_{21}\right)
$$

such that the corresponding mapping

$$
\delta \boldsymbol{\Theta}=\left(\delta \mathbf{u}, \delta \mathbf{v}, \delta \mathbf{w}, \delta \mathbf{V}, \delta \mathbf{W}, \delta \boldsymbol{\Lambda}, \delta \mathbf{R}_{i}\right) .
$$

given by (3.12) and (3.13), satisfies the equations

$$
\begin{gathered}
\delta \mathbf{u}(\boldsymbol{\xi})=\chi_{i}(\boldsymbol{\xi}) \frac{\partial}{\partial \xi_{i}}(\boldsymbol{\xi}+\mathbf{u}(\boldsymbol{\xi})) \Leftrightarrow \boldsymbol{\chi}=\mathbf{V}^{\top} \delta \mathbf{u}, \\
\delta \mathbf{v}=\chi_{i} \frac{\partial}{\partial \xi_{i}} \mathbf{v}+\mathbf{V} \boldsymbol{\mu}+\boldsymbol{\Lambda} \boldsymbol{\lambda} \\
\delta \mathbf{w}=\chi_{i} \frac{\partial}{\partial \xi_{i}} \mathbf{W}+\mathbf{W} \boldsymbol{\lambda} \\
\delta \mathbf{W}=\chi_{i} \frac{\partial}{\partial \xi_{i}} \mathbf{W}+\mathbf{W} \boldsymbol{\Gamma}
\end{gathered}
$$

and

$$
\begin{gathered}
\delta \mathbf{V}=\chi_{i} \frac{\partial}{\partial \xi_{i}} \mathbf{V}-\mathbf{V} \nabla_{\xi} \boldsymbol{\chi} \\
\delta \boldsymbol{\Lambda}=\chi_{i} \frac{\partial}{\partial \xi_{i}} \boldsymbol{\Lambda}+\mathbf{V} \nabla_{\xi}(\mathbf{J} \boldsymbol{\lambda})+\boldsymbol{\lambda}^{\top} \mathbf{R}-\boldsymbol{\Lambda} \boldsymbol{\Gamma} \\
\delta \mathbf{R}_{i}=\chi_{p} \frac{\partial}{\partial \xi_{p}} \mathbf{R}_{i}+\mathbf{R}_{i} \boldsymbol{\Gamma}+\left(\mathbf{R}_{i} \boldsymbol{\Gamma}\right)^{\top}+V_{i k} \frac{\partial}{\partial \xi_{k}}(\mathbf{J} \boldsymbol{\Gamma}),
\end{gathered}
$$


where $\boldsymbol{\lambda}^{\top} \mathbf{R}$ is $(n-1) \times 2$ matrix with the entries $\left(\boldsymbol{\lambda}^{\top} \mathbf{R}\right)_{i j}=R_{i, j p} \lambda_{p}$. Recall that by definition of $\Upsilon$,

$$
\boldsymbol{\mu}=\boldsymbol{\nu}+\nabla \psi_{0}
$$

Notice that relation (6B)-(7B) coincide with desired relations (3.19a) - (3.19g) in Theorem 3.2. In order to obtain the full system of relations (3.19) we have supplement (6B)-(7B) with the expression (3.19h) for $\delta \boldsymbol{\beta}$ and $\delta \varphi_{0}$ :

$$
\delta \boldsymbol{\beta}=\boldsymbol{\nu}, \quad \delta \varphi_{0}=\psi_{0}+w_{2} \delta w_{1}+\chi_{i} \frac{\partial}{\partial \xi_{i}} \varphi_{0}-w_{2} \chi_{i} \frac{\partial}{\partial \xi_{i}} w_{1}-\boldsymbol{\nu} \cdot \mathbf{u},
$$

where $\boldsymbol{\nu}$ and $\psi_{0}$ are the components of the given vector $\boldsymbol{\Upsilon}$. The system of equalities (6B) $-(8 \mathrm{~B})$ is equivalent to (3.19). It is important to note that the left hand sides of these equalities are the components of vector fields $\delta \boldsymbol{\varphi}$ and $\delta \Theta$. They are not independent and should satisfy relations (3.13) which constitutes the connection between $\delta \Theta \delta \boldsymbol{\varphi}$. Hence, relations (3.13) give the extra five equations

$$
\begin{gathered}
\delta \mathbf{V}=-\mathbf{V} \nabla_{\xi} \delta \mathbf{u} \mathbf{V} \\
\delta W_{22}=\frac{1}{W_{11}}\left(W_{12} \delta W_{21}+W_{21} \delta W_{12}-W_{22} \delta W_{11}\right), \\
\delta \mathbf{v}=\delta \beta+\mathbf{V}\left(\nabla \delta \varphi_{0}-w_{2} \nabla \delta w_{1}-\delta w_{2} \nabla w_{1}\right)+\delta \mathbf{V}\left(\nabla \varphi_{0}-w_{2} \nabla w_{1}\right), \\
\delta \boldsymbol{\Lambda}=-\delta \mathbf{V} \nabla \mathbf{w} \mathbf{J}-\mathbf{V} \nabla(\delta \mathbf{w}) \mathbf{J} \mathbf{W}-\mathbf{W} \nabla \mathbf{w} \mathbf{J} \delta \mathbf{W} \\
\delta \mathbf{R}_{i}=-\delta V_{i k} \frac{\partial}{\partial \xi_{k}}\left(\mathbf{W}^{\top}\right) \mathbf{J} \mathbf{W}-V_{i k} \frac{\partial}{\partial \xi_{k}}\left(\delta \mathbf{W}^{\top}\right) \mathbf{J} \mathbf{W}-V_{i k} \frac{\partial}{\partial \xi_{k}}\left(\mathbf{W}^{\top}\right) \mathbf{J} \delta \mathbf{W} .
\end{gathered}
$$

The obtained extended system (6B)-(9B) is overdetermined and we have to prove that it satisfies compatibility conditions. The assertion (i) of Theorem 3.2 will be proved if we prove that system (9B) is a consequence of equations $(6 \mathrm{~B})-(\underline{8 B})$. The proof falls into five steps.

Step 1. Let us show that equalities $(6 \mathrm{~B})$ and $(7 \mathrm{~B})$ yield (9Ba). We begin with the observation that equality (6Ba) along with relation (3.4a) in Theorem 3.1 implies $\boldsymbol{\chi}=\mathbf{V}^{\top} \delta \mathbf{u}$. From this we obtain

$$
\chi_{k} \frac{\partial}{\partial \xi_{k}} \mathbf{V}=V_{n k} \delta u_{n} \frac{\partial}{\partial \xi_{k}} \mathbf{V}
$$


Next we have

$$
\{\mathbf{V} \nabla \boldsymbol{\chi}\}_{i j}=V_{i k} \frac{\partial}{\partial \xi_{k}}\left(V_{n j} \delta u_{n}\right)=V_{i k} \frac{\partial V_{n j}}{\partial \xi_{k}} \delta u_{n}+V_{i k} V_{n j} \frac{\partial \delta u_{n}}{\partial \xi_{k}}
$$

Combining this result with $(10 \mathrm{~B})$ we arrive at

$$
\left\{\chi_{k} \frac{\partial \mathbf{V}}{\partial \xi_{k}}-\mathbf{V} \nabla \boldsymbol{\chi}\right\}_{i j}=\left(\frac{V_{n k} \partial V_{i j}}{\partial \xi_{k}}-V_{i k} \frac{\partial V_{n j}}{\partial \xi_{k}}\right)-V_{i k} V_{n j} \frac{\partial \delta u_{n}}{\partial \xi_{k}} .
$$

Next notice that $V_{n k} \partial / \partial \xi_{k}=\partial / \partial x_{n}$ and $V_{i j}=\partial \xi_{j} / \partial x_{i}$. It follows that

$$
V_{n k} \frac{\partial V_{i j}}{\partial \xi_{k}}-V_{i k} \frac{\partial V_{n j}}{\partial \xi_{k}}=\frac{\partial}{\partial x_{n}}\left(\frac{\partial \xi_{j}}{\partial x_{i}}\right)-\frac{\partial}{\partial x_{i}}\left(\frac{\partial \xi_{j}}{\partial x_{n}}\right)=0
$$

Substituting this equality into (11B) we arrive at the equality

$$
\chi_{k} \frac{\partial \mathbf{V}}{\partial \xi_{k}}-\mathbf{V} \nabla \boldsymbol{\xi}=-\mathbf{V} \nabla \delta \mathbf{u} \mathbf{V}
$$

which along with (7Ba) implies (9Ba).

Step 2. Let us prove that (6B) and (7B) imply (9Bb). Notice that $(9 \mathrm{Bb})$ is equivalent to the equality

$$
\delta \mathbf{W}^{\top} \mathbf{J W}+\mathbf{W}^{\top} \mathbf{J} \delta \mathbf{W}=0 .
$$

It follows from (6Bd $)$ that

$$
\begin{array}{r}
\delta \mathbf{W}^{\top} \mathbf{J W}+\mathbf{W}^{\top} \mathbf{J} \delta \mathbf{W}=\chi_{i}\left(\frac{\partial}{\partial \xi_{i}} \mathbf{W}^{\top} \mathbf{J W}+\mathbf{W}^{\top} \mathbf{J} \frac{\partial}{\partial \xi_{i}} \mathbf{W}\right)+ \\
(\mathbf{W} \boldsymbol{\Gamma})^{\top} \mathbf{J W}+\mathbf{W}^{\top} \mathbf{J W} \boldsymbol{\Gamma} .
\end{array}
$$

Next, relation (3.4b) in Theorem 3.1 yields $\mathbf{W}^{\top} \mathbf{J W}=\mathbf{J}$. It follows that

$$
\frac{\partial}{\partial \xi_{i}} \mathbf{W}^{\top} \mathbf{J} \mathbf{W}+\mathbf{W}^{\top} \mathbf{J} \frac{\partial}{\partial \xi_{i}} \mathbf{W}=0
$$

and

$$
(\mathbf{W} \boldsymbol{\Gamma})^{\top} \mathbf{J W}+\mathbf{W}^{\top} \mathbf{J W} \boldsymbol{\Gamma}=\boldsymbol{\Gamma}^{\top} \mathbf{J}+\mathbf{J} \boldsymbol{\Gamma}=\mathbf{J} \boldsymbol{\Gamma}-(\mathbf{J} \boldsymbol{\Gamma})^{\top}=0
$$

since the matrix $\mathbf{J} \boldsymbol{\Gamma}$ is symmetric and $\mathbf{J}^{\top}=-\mathbf{J}$. Substituting these relations into (13B) we obtain (12B) and the assertion follows. 
Step 3. Let us prove that equalities $(6 \mathrm{~B})-(8 \mathrm{~B})$ imply (9Bc). It is necessary to prove that the right hand side of (6Bb) coincide with the right hand side of $(\overline{\mathrm{BC}})$. Let us calculate step by step all terms in the right hand side of (6Bb). Relation (6Ba) and the identity $V_{i k} \partial / \partial \xi_{k}=\partial / \partial x_{i}$ imply

$$
\chi_{i} \frac{\partial}{\partial \xi_{i}} \mathbf{v}=\delta u_{i} \frac{\partial}{\partial x_{i}} \mathbf{v}
$$

On the other hand, relation (3.4f) in Theorem 3.1 yields

$$
\mathbf{v}=\boldsymbol{\beta}+\nabla_{x} \varphi_{0}-w_{2} \nabla_{x} w_{1}
$$

Combining these results we arrive at

$$
\chi_{i} \frac{\partial}{\partial \xi_{i}} \mathbf{v}=\delta u_{i} \frac{\partial}{\partial x_{i}}\left(\nabla_{x} \varphi_{0}-w_{2} \nabla_{x} w_{1}\right) .
$$

Next, relation (3.4c) in Theorem 3.1 implies

$$
\Lambda=-\mathbf{V} \nabla_{\xi} \mathbf{w} \mathbf{J} \mathbf{W}=-\nabla_{x} \mathbf{w} \mathbf{J W} .
$$

In its turn, it follows from (6.68) that

$$
\boldsymbol{\lambda}=\mathbf{W}^{-1} \delta \mathbf{w}-\chi_{i} \mathbf{W}^{-1} \frac{\partial}{\partial \xi_{i}} \mathbf{w}=\mathbf{W}^{-1} \delta \mathbf{w}-\delta u_{i} \mathbf{W}^{-1} \frac{\partial}{\partial x_{i}} \mathbf{w} .
$$

Thus we get

$$
\begin{array}{r}
\boldsymbol{\Lambda} \boldsymbol{\lambda}=\nabla_{x} \mathbf{w} \mathbf{J}\left(\delta u_{i} \frac{\partial}{\partial x_{i}} \mathbf{w}\right)-\nabla_{x} \mathbf{w} \mathbf{J} \delta \mathbf{w}=\nabla_{x}\left(w_{2} \delta w_{1}\right)- \\
\left(\delta w_{2} \nabla_{x} w_{1}+w_{2} \nabla_{x} \delta w_{1}\right)+\left(\delta u_{i} \frac{\partial}{\partial x_{i}} w_{2}\right) \nabla_{x} w_{1}-\left(\delta u_{i} \frac{\partial}{\partial x_{i}} w_{1}\right) \nabla_{x} w_{2}
\end{array}
$$

Combining this result with $(15 \mathrm{~B})$ we arrive at

$$
\begin{array}{r}
\chi_{i} \frac{\partial}{\partial \xi_{i}} \mathbf{v}+\boldsymbol{\Lambda} \boldsymbol{\lambda}=\nabla_{x}\left(w_{2} \delta w_{1}\right)-\left(\delta w_{2} \nabla_{x} w_{1}+w_{2} \nabla_{x} \delta w_{1}\right)+ \\
\delta u_{i} \nabla_{x}\left(\frac{\partial}{\partial x_{i}} \varphi_{0}-w_{2} \frac{\partial}{\partial x_{i}} w_{1}\right) .
\end{array}
$$

Next notice that

$$
\begin{gathered}
\delta u_{i} \nabla_{x}\left(\frac{\partial}{\partial x_{i}} \varphi_{0}-w_{2} \frac{\partial}{\partial x_{i}} w_{1}\right)= \\
\nabla_{x}\left(\delta u_{i}\left(\frac{\partial}{\partial x_{i}} \varphi_{0}-w_{2} \frac{\partial}{\partial x_{i}} w_{1}\right)\right)-\nabla_{x}(\delta \mathbf{u})\left(\nabla_{x} \varphi_{0}-w_{2} \nabla_{x} w_{1}\right) .
\end{gathered}
$$


Hence we can rewrite $(\underline{17 \mathrm{~B}})$ in the form

$$
\begin{array}{r}
\chi_{i} \frac{\partial}{\partial \xi_{i}} \mathbf{v}+\boldsymbol{\Lambda} \boldsymbol{\lambda}=\nabla_{x}\left(w_{2} \delta w_{1}+\delta u_{i} \frac{\partial}{\partial x_{i}} \varphi_{0}-\delta u_{i} w_{2} \frac{\partial}{\partial x_{i}} w_{1}\right)- \\
\left(\delta w_{2} \nabla_{x} w_{1}+w_{2} \nabla_{x} \delta w_{1}\right)-\nabla_{x}(\delta \mathbf{u})\left(\nabla_{x} \varphi_{0}-w_{2} \nabla_{x} w_{1}\right) .
\end{array}
$$

On the other hand, the identity $\nabla_{x}=\mathbf{V} \nabla_{\xi}$ and relation (9Ba), which we have been proved in Step 1, imply

$$
\nabla_{x}(\delta \mathbf{u})\left(\nabla_{x} \varphi_{0}-w_{2} \nabla_{x} w_{1}\right)=-\delta \mathbf{V}\left(\nabla_{\xi} \varphi_{0}-w_{2} \nabla_{\xi} w_{1}\right)
$$

Moreover, as it was mentioned above, we have $\delta u_{k} \partial / \partial x_{k}=\chi_{k} \partial / \partial \xi_{k}$. It follows from this and (18B) that

$$
\begin{array}{r}
\chi_{i} \frac{\partial}{\partial \xi_{i}} \mathbf{v}+\boldsymbol{\Lambda} \boldsymbol{\lambda}=\mathbf{V} \nabla_{\xi}\left(w_{2} \delta w_{1}+\chi_{i} \frac{\partial}{\partial \xi_{i}} \varphi_{0}-\chi_{i} w_{2} \frac{\partial}{\partial \xi_{i}} w_{1}\right)- \\
\mathbf{V}\left(\delta w_{2} \nabla_{\xi} w_{1}+w_{2} \nabla_{\xi} \delta w_{1}\right)+\delta \mathbf{V}\left(\nabla_{\xi} \varphi_{0}-w_{2} \nabla_{\xi} w_{1}\right) .
\end{array}
$$

Let us consider the term $\mathbf{V} \boldsymbol{\mu}$ in expression (6Bb) for $\mathbf{v}$. It follows from (7Bd) that

$$
\mathbf{V} \boldsymbol{\mu}=\mathbf{V} \boldsymbol{\nu}+\mathbf{V} \nabla_{\xi} \psi_{0} .
$$

Next notice that

$\mathbf{V} \boldsymbol{\nu}=\mathbf{V} \nabla_{\xi}(\boldsymbol{\nu} \cdot \boldsymbol{\xi})=\nabla_{x}(\boldsymbol{\nu} \cdot \boldsymbol{\xi})=\nabla_{x}(\boldsymbol{\nu} \mathbf{x}-\boldsymbol{\nu} \cdot \mathbf{u})=\boldsymbol{\nu}-\nabla_{x}(\boldsymbol{\nu} \cdot \mathbf{u})=\boldsymbol{\nu}-\mathbf{V} \nabla_{\xi}(\boldsymbol{\nu} \cdot \mathbf{u})$.

Thus we get

$$
\mathbf{V} \boldsymbol{\mu}=\boldsymbol{\nu}+\mathbf{V} \nabla_{\xi}\left(\psi_{0}-\boldsymbol{\nu} \cdot \mathbf{u}\right) .
$$

Combining this result with (19B) and recalling formulae (6Bb) for $\delta \mathbf{v}$ and (8B) for $\varphi_{0}$ we finally obtain

$$
\begin{array}{r}
\delta \mathbf{v}=\chi_{i} \frac{\partial}{\partial \xi_{i}} \mathbf{v}+\boldsymbol{\Lambda} \boldsymbol{\lambda}+\mathbf{V} \boldsymbol{\mu}=\boldsymbol{\nu}+\mathbf{V} \nabla \delta \varphi_{0}- \\
\mathbf{V}\left(\delta w_{2} \nabla_{\xi} w_{1}+w_{2} \nabla_{\xi} \delta w_{1}\right)+\delta \mathbf{V}\left(\nabla_{\xi} \varphi_{0}-w_{2} \nabla_{\xi} w_{1}\right),
\end{array}
$$

which obviously yields (9Bc). 
Step 4. Let us prove that equalities (6B) $-(7 \mathrm{~B})$ imply (9Bd $)$. It follows from $(7 \mathrm{Bb})$ that

$$
\delta \boldsymbol{\Lambda}=\chi_{i} \frac{\partial \boldsymbol{\Lambda}}{\partial \xi_{i}}+\mathbf{V} \nabla_{\xi}(\mathbf{J} \boldsymbol{\lambda})+\boldsymbol{\lambda}^{\top} \mathbf{R}-\boldsymbol{\Lambda} \boldsymbol{\Gamma} .
$$

Arguing as in the proof of (14B) and using the identity $V_{i k} \partial / \partial \xi_{k}=\partial / \partial x_{i}$ we obtain

$$
\chi_{i} \frac{\partial \boldsymbol{\Lambda}}{\partial \xi_{i}}=\delta u_{i} \frac{\partial \boldsymbol{\Lambda}}{\partial x_{i}}, \quad \mathbf{V} \nabla_{\xi}(\mathbf{J} \boldsymbol{\lambda})=\nabla_{x}(\mathbf{J} \boldsymbol{\lambda}) .
$$

Thus we get

$$
\delta \boldsymbol{\Lambda}=\delta u_{i} \frac{\partial \boldsymbol{\Lambda}}{\partial x_{i}}+\nabla_{x}(\mathbf{J} \boldsymbol{\lambda})+\boldsymbol{\lambda}^{\top} \mathbf{R}-\boldsymbol{\Lambda} \boldsymbol{\Gamma} .
$$

Next, it follows from the relation $(3.4 \mathrm{~d})$ in Theorem 3.1 that

$$
\mathbf{R}_{i}=-V_{i k} \frac{\partial \mathbf{W}^{\top}}{\partial \xi_{k}} \mathbf{J} \mathbf{W}=-\frac{\partial \mathbf{W}^{\top}}{\partial x_{i}} \mathbf{J} \mathbf{W}
$$

Here $\boldsymbol{\lambda}^{\top} \mathbf{R}$ is $(n-1) \times 2$-matrix with the rows $\boldsymbol{\lambda}^{\top} \mathbf{R}_{i}, 1 \leq i \leq n-1$. It follows from (23B) that

$$
\boldsymbol{\lambda}^{\top} \mathbf{R}_{i}=-\boldsymbol{\lambda}^{\top} \frac{\partial \mathbf{W}^{\top}}{\partial x_{i}} \mathbf{J} \mathbf{W}=-\frac{\partial}{\partial x_{i}}\left(\boldsymbol{\lambda}^{\top} \mathbf{W}^{\top}\right) \mathbf{J W}+\frac{\partial \boldsymbol{\lambda}^{\top}}{\partial x_{i}} \mathbf{W}^{\top} \mathbf{J} \mathbf{W}
$$

Noting that in view of relation (3.4b) in Theorem 3.1, we have $\mathbf{W}^{\top} \mathbf{J W}=$ $\mathbf{J}=-\mathbf{J}^{\top}$, we obtain the expression for the rows $\boldsymbol{\lambda}^{\top} \mathbf{R}_{i}$ of the matrix $\boldsymbol{\lambda}^{\top} \mathbf{R}$

$$
\boldsymbol{\lambda}^{\top} \mathbf{R}_{i}=-\frac{\partial}{\partial x_{i}}(\mathbf{W} \boldsymbol{\lambda})^{\top} \mathbf{J} \mathbf{W}-\frac{\partial}{\partial x_{i}}(\mathbf{J} \boldsymbol{\lambda})^{\top}
$$

which leads to

$$
\boldsymbol{\lambda}^{\top} \mathbf{R}=-\nabla_{x}(\mathbf{W} \boldsymbol{\lambda}) \mathbf{J} \mathbf{W}-\nabla_{x}(\mathbf{J} \boldsymbol{\lambda}) .
$$

On the other hand, equality (6.68) yields

$$
\boldsymbol{\lambda}=\mathbf{W}^{-1}\left(\delta \mathbf{w}-\chi_{i} \frac{\partial \mathbf{w}}{\partial \xi_{i}}\right)=\mathbf{W}^{-1}\left(\delta \mathbf{w}-\delta u_{i} \frac{\partial \mathbf{w}}{\partial x_{i}}\right)
$$

Substituting this relation into the latter identity we arrive at

$$
\boldsymbol{\lambda}^{\top} \mathbf{R}=-\nabla_{x}(\delta \mathbf{w}) \mathbf{J W}+\nabla_{x}\left(\delta u_{i} \frac{\partial \mathbf{w}}{\partial x_{i}}\right) \mathbf{J W}-\nabla_{x}(\mathbf{J} \boldsymbol{\lambda}) .
$$


Let us calculate $\Lambda \boldsymbol{\Gamma}$. Equalities (6Bd), the identity $\mathbf{V} \nabla_{\xi}=\nabla_{x}$, and equality (9Ba), which we proved in Step 1, implies

$$
\boldsymbol{\Gamma}=\mathbf{W}^{-1} \delta \mathbf{W}-\chi_{i} \mathbf{W}^{-1} \frac{\partial \mathbf{W}}{\partial \xi_{i}}=\mathbf{W}^{-1} \delta \mathbf{W}-\delta u_{i} \mathbf{W}^{-1} \frac{\partial \mathbf{W}}{\partial x_{i}} .
$$

On the other hand, equality (3.4c) yields $\boldsymbol{\Lambda}=-\nabla_{x}(\mathbf{w}) \mathbf{J W}$. Thus we get

$$
\boldsymbol{\Lambda} \boldsymbol{\Gamma}=-\nabla_{x}(\mathbf{w}) \mathbf{J} \delta \mathbf{W}+\delta u_{i}\left(\nabla_{x} \mathbf{w}\right) \mathbf{J} \frac{\partial \mathbf{W}}{\partial x_{i}} .
$$

Substituting this result along with $(24 \mathrm{~B})$ into $(22 \mathrm{~B})$ we arrive at

$$
\begin{array}{r}
\delta \boldsymbol{\Lambda}=\delta u_{i}\left(\frac{\partial \boldsymbol{\Lambda}}{\partial x_{i}}+\nabla_{x} \mathbf{w} \mathbf{J} \frac{\partial \mathbf{W}}{\partial x_{i}}\right)+\nabla_{x}\left(\delta u_{i} \frac{\partial \mathbf{w}}{\partial x_{i}}\right) \mathbf{J W}- \\
\nabla_{x}(\delta \mathbf{w}) \mathbf{J W}-\nabla_{x} \mathbf{w} \mathbf{J}(\delta \mathbf{W}) .
\end{array}
$$

Let us calculate separately the term containing $\delta u_{i}$. We begin with the observation that

$$
\nabla_{x}\left(\delta u_{i} \frac{\partial \mathbf{w}}{\partial x_{i}}\right)=\delta u_{i} \frac{\partial}{\partial x_{i}} \nabla_{x} \mathbf{w}+\nabla_{x}(\delta \mathbf{u}) \nabla_{x} \mathbf{w} .
$$

Thus we get

$$
\nabla_{x}\left(\delta u_{i} \frac{\partial \mathbf{w}}{\partial x_{i}}\right) \mathbf{J W}=\delta u_{i} \frac{\partial}{\partial x_{i}}\left(\nabla_{x} \mathbf{w}\right) \mathbf{J W}+\nabla_{x}(\delta \mathbf{u}) \nabla_{x} \mathbf{w} \mathbf{J W} .
$$

Next, the identity $\boldsymbol{\Lambda}=-\nabla_{x} \mathbf{w} \mathbf{J} \mathbf{W}$ implies

$$
\delta u_{i} \frac{\partial \boldsymbol{\Lambda}}{\partial x_{i}}=-\delta u_{i} \frac{\partial}{\partial x_{i}}\left(\nabla_{x} \mathbf{w}\right) \mathbf{J W}-\delta u_{i} \nabla_{x} \mathbf{w} \mathbf{J} \frac{\partial \mathbf{W}}{\partial x_{i}}
$$

Combining this result with $(27 \mathrm{~B})$ we obtain

$$
\delta u_{i}\left(\frac{\partial}{\partial x_{i}} \boldsymbol{\Lambda}+\nabla_{x} \mathbf{w} \mathbf{J} \frac{\partial \mathbf{W}}{\partial x_{i}}\right)+\nabla_{x}\left(\delta u_{i} \frac{\partial \mathbf{w}}{\partial x_{i}}\right) \mathbf{J} \mathbf{W}=\nabla_{x}(\delta \mathbf{u}) \nabla_{x} \mathbf{w} \mathbf{J W} .
$$

Substituting this result in (26B) we obtain

$$
\delta \boldsymbol{\Lambda}=-\nabla_{x}(\delta \mathbf{w}) \mathbf{J W}-\nabla_{x} \mathbf{w} \mathbf{J} \delta \mathbf{W}+\nabla_{x}(\delta \mathbf{u}) \nabla_{x} \mathbf{w} \mathbf{J W} .
$$

Next, relation (9Ba), which was proved in Step 1, and the identity $\mathbf{V} \nabla_{\xi}=\nabla_{x}$ imply

$$
\begin{aligned}
\nabla_{x}(\delta \mathbf{u}) \nabla_{x} \mathbf{w} \mathbf{J W} & =\left(\mathbf{V} \nabla_{\xi} \delta \mathbf{u}\right) \nabla_{x} \mathbf{w} \mathbf{J W}=-\delta \mathbf{V} \nabla_{x} \mathbf{w} \mathbf{J} \mathbf{W} \\
\nabla_{x}(\delta \mathbf{w}) & =\mathbf{V} \nabla_{\xi}(\delta \mathbf{w}), \quad \nabla_{x} \mathbf{w}=\mathbf{V} \nabla_{\xi} \mathbf{w} .
\end{aligned}
$$

Substituting these equalities into (28B) gives desired relation (9Bd) and the assertion follows. 
Step 5. It remains to prove that equalities (6B)-(7B) imply (9Be). We proved yet that $\delta \mathbf{V}$ satisfies relation (9Ba), i.e.,

$$
\delta V_{i k}=-V_{i n}\left(\frac{\partial}{\partial \xi_{n}} \delta u_{p}\right) V_{p k}
$$

It follows from this (9Ba) and the identity $\mathbf{V} \nabla_{\xi}=\nabla_{x}$ that

$$
\delta V_{i k} \partial / \partial \xi_{k}=-V_{i n}\left(\frac{\partial}{\partial \xi_{n}} \delta u_{p}\right) V_{p k} \partial / \partial \xi_{k}=-\left(\frac{\partial}{\partial x_{i}} \delta u_{p}\right) \partial / \partial x_{p}
$$

It follows from this that relation (9Be $)$ can be rewritten in the equivalent form

$$
\delta \mathbf{R}_{i}=\left(\frac{\partial}{\partial x_{i}} \delta u_{k}\right) \frac{\partial}{\partial x_{k}}\left(\mathbf{W}^{\top}\right) \mathbf{J} \mathbf{W}-\frac{\partial}{\partial x_{i}}\left(\delta \mathbf{W}^{\top}\right) \mathbf{J} \mathbf{W}-\frac{\partial}{\partial x_{i}}\left(\mathbf{W}^{\top}\right) \mathbf{J} \delta \mathbf{W} .
$$

Hence it suffices to prove that relations $(6 \mathrm{~B})-(7 \mathrm{~B})$ yields $(29 \mathrm{~B})$. To this end notice that relation (6Ba) and the identity $V_{i k} \partial / \partial \xi_{k}=\partial / \partial x_{i}$ imply

$$
\chi_{k} \frac{\partial}{\partial \xi_{k}} \mathbf{R}_{i}=\delta u_{k} \frac{\partial}{\partial x_{k}} \mathbf{R}_{i}, \quad V_{i k} \frac{\partial}{\partial \xi_{k}}(\mathbf{J} \boldsymbol{\Gamma})=\frac{\partial}{\partial x_{i}}(\mathbf{J} \boldsymbol{\Gamma}) .
$$

Using these identities we can rewrite relation (7Bc) in the form

$$
\delta \mathbf{R}_{i}=\delta u_{k} \frac{\partial}{\partial x_{k}} \mathbf{R}_{i}+\frac{\partial}{\partial x_{i}}(\mathbf{J} \boldsymbol{\Gamma})+\mathbf{R}_{i} \boldsymbol{\Gamma}+\left(\mathbf{R}_{i} \boldsymbol{\Gamma}\right)^{\top} .
$$

Let us calculate all terms in the right hand side. It follows from (6Bd $)$ and (6Ba) that

$$
\delta \mathbf{W}=\chi_{i} \frac{\partial}{\partial \xi_{i}} \mathbf{W}+\mathbf{W} \boldsymbol{\Gamma}=\delta u_{k} \frac{\partial}{\partial x_{k}} \mathbf{W}+\mathbf{W} \boldsymbol{\Gamma}
$$

or equivalently

$$
\boldsymbol{\Gamma}=\mathbf{W}^{-1} \delta \mathbf{W}-\delta u_{k} \mathbf{W}^{-1} \frac{\partial}{\partial x_{k}} \mathbf{W}
$$

Since $\mathbf{J W}^{-1}=\mathbf{W}^{\top} \mathbf{J}$ we conclude from this that

$$
\mathbf{J} \boldsymbol{\Gamma}=\mathbf{W}^{\top} \mathbf{J} \delta \mathbf{W}-\delta u_{k} \mathbf{W}^{\top} \frac{\partial}{\partial x_{k}} \mathbf{W},
$$


which leads to the equality

$$
\begin{array}{r}
\frac{\partial}{\partial x_{i}}(\mathbf{J} \boldsymbol{\Gamma})=\frac{\partial \mathbf{W}^{\top}}{\partial x_{i}} \mathbf{J} \delta \mathbf{W}+\mathbf{W}^{\top} \mathbf{J} \frac{\partial \delta \mathbf{W}}{\partial x_{i}}- \\
\delta u_{k}\left(\frac{\partial \mathbf{W}^{\top}}{\partial x_{i}} \mathbf{J} \frac{\partial \mathbf{W}}{\partial x_{k}}+\mathbf{W}^{\top} \mathbf{J} \frac{\partial^{2} \mathbf{W}}{\partial x_{i} \partial x_{k}}\right)-\frac{\partial \delta u_{k}}{\partial x_{i}} \mathbf{W}^{\top} \mathbf{J} \frac{\partial \mathbf{W}}{\partial x_{k}} .
\end{array}
$$

Next, equality (23B) implies

$$
\delta u_{k} \frac{\partial}{\partial x_{k}} \mathbf{R}_{i}=-\delta u_{k}\left(\frac{\partial^{2} \mathbf{W}^{\top}}{\partial x_{i} \partial x_{k}} \mathbf{J W}+\frac{\partial \mathbf{W}^{\top}}{\partial x_{i}} \mathbf{J} \frac{\partial \mathbf{W}}{\partial x_{k}}\right)
$$

Notice that relations (23B $)$ and $(\underline{25 \mathrm{~B}})$ imply

$$
\begin{array}{r}
\mathbf{R}_{i} \boldsymbol{\Gamma}+\left(\mathbf{R}_{i} \boldsymbol{\Gamma}\right)^{\top}=\delta \mathbf{W}^{\top} \mathbf{J} \frac{\partial \mathbf{W}}{\partial x_{i}}-\frac{\partial \mathbf{W}^{\top}}{\partial x_{i}} \mathbf{J} \delta \mathbf{W}^{\top}+ \\
\delta u_{k}\left(\frac{\partial \mathbf{W}^{\top}}{\partial x_{i}} \mathbf{J} \frac{\partial \mathbf{W}}{\partial x_{k}}-\frac{\partial \mathbf{W}^{\top}}{\partial x_{k}} \mathbf{J} \frac{\partial \mathbf{W}}{\partial x_{i}}\right) .
\end{array}
$$

Substituting (31B $-(\underline{34 \mathrm{~B}})$ into $(\underline{30 \mathrm{~B}})$ we obtain

$\delta \mathbf{R}_{i}=-\frac{\partial \delta u_{k}}{\partial x_{i}} \mathbf{W}^{\top} \mathbf{J} \frac{\partial \mathbf{W}}{\partial x_{k}}+\mathbf{W}^{\top} \mathbf{J} \frac{\partial \delta \mathbf{W}}{\partial x_{i}}+\delta \mathbf{W}^{\top} \mathbf{J} \frac{\partial \mathbf{W}}{\partial x_{i}}-\delta u_{k} \frac{\partial^{2}}{\partial x_{i} \partial x_{k}}\left(\mathbf{W}^{\top} \mathbf{J W}\right)$.

On the other hand, the identity $\mathbf{W}^{\top} \mathbf{J W}=\mathbf{J}$ implies

$$
\frac{\partial^{2}}{\partial x_{i} \partial x_{k}}\left(\mathbf{W}^{\top} \mathbf{J W}\right)=0, \quad \mathbf{W}^{\top} \mathbf{J} \frac{\partial \mathbf{W}}{\partial x_{k}}+\frac{\partial \mathbf{W}^{\top}}{\partial x_{k}} \mathbf{J W}=0 .
$$

Thus we get

$$
\delta \mathbf{R}_{i}=\frac{\partial \delta u_{k}}{\partial x_{i}} \frac{\partial \mathbf{W}^{\top}}{\partial x_{k}} \mathbf{J} \mathbf{W}+\mathbf{W}^{\top} \mathbf{J} \frac{\partial \delta \mathbf{W}}{\partial x_{i}}+\delta \mathbf{W}^{\top} \mathbf{J} \frac{\partial \mathbf{W}}{\partial x_{i}}
$$

Relation (9Bb), which was proved in Step 2, yields $\delta \mathbf{W}^{\top} \mathbf{J W}+\mathbf{W}^{\top} \mathbf{J} \delta \mathbf{W}=0$ differentiation both sides of this equality gives

$$
\mathbf{W}^{\top} \mathbf{J} \frac{\partial \delta \mathbf{W}}{\partial x_{i}}+\delta \mathbf{W}^{\top} \mathbf{J} \frac{\partial \mathbf{W}}{\partial x_{i}}=-\frac{\partial}{\partial x_{i}}\left(\delta \mathbf{W}^{\top}\right) \mathbf{J} \mathbf{W}-\frac{\partial}{\partial x_{i}}\left(\mathbf{W}^{\top}\right) \mathbf{J} \delta \mathbf{W} .
$$

Substituting this relation into (34B) we obtain desired identity (29B), and the assertion follows. 


\section{B.2 Proof of (ii).}

Choose an arbitrary analytic

$$
\boldsymbol{\varphi}=\left(\beta, \varphi_{0}, \mathbf{u}, \mathbf{w}, W_{11}, W_{12}, W_{21}\right)
$$

and consider the vector field

$$
\Theta(\varphi)=\left(\mathbf{u}, \mathbf{v}, \mathbf{w}, \mathbf{V}, \boldsymbol{\Lambda}, \mathbf{W}, \mathbf{R}_{i}\right)
$$

defined by (3.8). Let $\boldsymbol{\theta}$ be a corresponding canonical mapping defined by (3.1). Next choose an arbitrary vector field

$$
\delta \boldsymbol{\varphi}=\left(\delta \beta, \delta \varphi_{0}, \delta \mathbf{u}, \delta \mathbf{w}, \delta W_{11}, \delta W_{12}, \delta W_{21}\right) \in X_{\sigma, d-1}
$$

and the corresponding vector field

$$
\delta \boldsymbol{\Theta}=\left(\delta \mathbf{u}, \delta \mathbf{v}, \delta \mathbf{w}, \delta \mathbf{V}, \delta \mathbf{W}, \delta \boldsymbol{\Lambda}, \delta \mathbf{R}_{i}\right) .
$$

Let us consider the associated mapping

$$
\delta \boldsymbol{\theta}=\left(\begin{array}{c}
\delta \mathbf{u}(\xi) \\
\delta \mathbf{v}(\boldsymbol{\xi})+\delta \mathbf{V}(\boldsymbol{\xi}) \boldsymbol{\eta}+\delta \boldsymbol{\Lambda}(\boldsymbol{\xi}) \boldsymbol{\zeta}+\boldsymbol{\zeta}^{\top} \delta \mathbf{R}(\boldsymbol{\xi}) \boldsymbol{\zeta} \\
\delta \mathbf{w}(\boldsymbol{\xi})+\delta \mathbf{W}(\boldsymbol{\xi}) \boldsymbol{\zeta}
\end{array}\right)
$$

with the coefficients given by (3.13), i. e.,

$$
\begin{gathered}
\delta \mathbf{V}=-\mathbf{V} \delta \nabla_{\xi} \delta \mathbf{u} \mathbf{V}, \quad \delta W_{22}=\frac{1}{W_{11}}\left(W_{12} \delta W_{21}+W_{21} \delta W_{12}-W_{22} \delta W_{11}\right), \\
\delta \mathbf{W}=\left(\begin{array}{cc}
\delta W_{11} & \delta W_{12} \\
\delta W_{21} & \delta W_{22}
\end{array}\right), \\
\delta \mathbf{v}=\delta \beta+\mathbf{V}\left(\nabla \delta \varphi_{0}-w_{2} \nabla \delta w_{1}-\delta w_{2} \nabla w_{1}\right)+\delta \mathbf{V}\left(\nabla \varphi_{0}-w_{2} \nabla w_{1}\right), \\
\delta \boldsymbol{\Lambda}=\delta \mathbf{V} \nabla w \mathbf{J W}-\mathbf{V} \nabla(\delta \mathbf{W}) \mathbf{J} \mathbf{W}-\mathbf{W} \nabla \mathbf{W} \mathbf{J} \delta \mathbf{W} \\
\delta \mathbf{R}_{i}=-\delta V_{i k} \frac{\partial}{\partial \xi_{k}}\left(\mathbf{W}^{\top}\right) \mathbf{J} \mathbf{W}-V_{i k} \frac{\partial}{\partial \xi_{k}}\left(\delta \mathbf{W}^{\top}\right) \mathbf{J} \mathbf{W}-V_{i k} \frac{\partial}{\partial \xi_{k}}\left(\mathbf{W}^{\top}\right) \mathbf{J} \delta \mathbf{W}
\end{gathered}
$$

Our task is to find to find a vector field

$$
\boldsymbol{\Upsilon}=\left(\boldsymbol{\nu}, \psi_{0}, \boldsymbol{\chi}, \boldsymbol{\lambda}, \Gamma_{11}, \Gamma_{12}, \Gamma_{21}\right) \in X_{\sigma, d-1}(r) .
$$

such that the corresponding vector field

$$
\mathfrak{Z}:=\delta \boldsymbol{\Theta} \equiv D_{\varphi} \boldsymbol{\Theta}\left(\boldsymbol{\varphi}_{0}\right)[\boldsymbol{\Upsilon}],
$$




$$
\mathfrak{Z} \equiv\left(\boldsymbol{\chi}, \boldsymbol{\mu}, \boldsymbol{\lambda},-\nabla \boldsymbol{\chi}, \boldsymbol{\Gamma}, \nabla(\mathbf{J} \boldsymbol{\lambda}), \partial_{\xi_{i}}(\mathbf{J} \boldsymbol{\Gamma})\right)
$$

with

$$
\boldsymbol{\mu}=\boldsymbol{\nu}+\nabla \psi_{0}, \quad \operatorname{Tr} \boldsymbol{\Gamma}=0 .
$$

satisfies the relations (3.20), i.e.,

$$
\begin{aligned}
& \chi=\mathbf{V}^{\top} \delta \mathbf{u} \\
& \boldsymbol{\lambda}=\mathbf{W}^{-1} \delta \mathbf{w}-\chi_{i} \mathbf{W}^{-1} \frac{\partial}{\partial \xi_{i}} \mathbf{w} \\
& \boldsymbol{\Gamma}=\mathbf{W}^{-1} \delta \mathbf{W}-\chi_{i} \mathbf{W}^{-1} \frac{\partial}{\partial \xi_{i}} \mathbf{W}, \\
& \boldsymbol{\mu}=\mathbf{V}^{-1}\left(\delta \mathbf{W}+\chi_{i} \frac{\partial}{\partial \xi_{i}} \mathbf{v}-\boldsymbol{\Lambda} \boldsymbol{\lambda}\right) \\
& \nabla \psi_{0}=\boldsymbol{\mu}-\boldsymbol{\nu}, \quad \boldsymbol{\nu}=\frac{1}{(2 \pi)^{n-1}} \int_{\mathbb{T}^{n-1}} \boldsymbol{\mu} d \boldsymbol{\xi}=\delta \boldsymbol{\beta} .
\end{aligned}
$$

In order to prove (44B), we consider the associated canonical mapping

$$
\boldsymbol{\pi}=\left(\begin{array}{c}
\boldsymbol{\chi}(\boldsymbol{\xi}) \\
\boldsymbol{\mu}-\left(\nabla_{\xi} \boldsymbol{\chi}\right) \boldsymbol{\eta}+\nabla_{\xi}(\mathbf{J} \boldsymbol{\lambda}) \boldsymbol{\zeta}+\frac{1}{2} \nabla_{\xi}(\mathbf{J} \boldsymbol{\Gamma} \cdot \boldsymbol{\zeta}) \\
\boldsymbol{\lambda}+\boldsymbol{\Gamma} \boldsymbol{\zeta}
\end{array}\right)
$$

Notice that relations (44B) can be written in the form

$$
\delta \boldsymbol{\theta}=\boldsymbol{\theta}^{\prime} \boldsymbol{\pi} .
$$

Here the Jacobi matrix $\boldsymbol{\theta}^{\prime}$ is defined by $(\underline{1 \mathrm{~A}})-(2 \mathrm{~A})$. Since $\boldsymbol{\theta}$ and $\delta \boldsymbol{\theta}$ are given, it suffices to prove that a solution $\boldsymbol{\pi}$ to (46B) admits representation (45B). Since, in view of (3.3), the matrix $\boldsymbol{\theta}^{\prime}$ is symplectic, a solution of (46B) is given by

$$
\boldsymbol{\pi}=-\mathbf{J}_{2 n} \boldsymbol{\vartheta}^{\prime \top} \mathbf{J}_{2 n} \delta \boldsymbol{\vartheta} .
$$

Here the matrix $\mathbf{J}_{2 n}$ is defined by (3.3). Substituting (39B) into (47B) we conclude that the mapping $\boldsymbol{\pi}$ admits the representation

$$
\boldsymbol{\pi}=\left(\begin{array}{c}
\boldsymbol{\chi}(\boldsymbol{\xi}) \\
\boldsymbol{\mu}(\boldsymbol{\xi})+\mathbf{A}(\boldsymbol{\xi}) \boldsymbol{\eta}+\mathbf{B}(\boldsymbol{\xi}) \boldsymbol{\zeta}+\frac{1}{2} \boldsymbol{\zeta}^{\top} \boldsymbol{C}(\boldsymbol{\xi}) \boldsymbol{\zeta} \\
\boldsymbol{\lambda}(\boldsymbol{\xi})+\boldsymbol{\Gamma}(\boldsymbol{\xi}) \boldsymbol{\zeta}
\end{array}\right)
$$


Here $\boldsymbol{\chi}, \boldsymbol{\mu}, \boldsymbol{\lambda}$ are $2 \pi$ - periodic vector fields, $\mathbf{A}, \mathbf{B}$ and $\boldsymbol{\Gamma}$ are $2 \pi$ periodic matrices, and a vector-valued function $\boldsymbol{\zeta}^{\top} \boldsymbol{C}(\boldsymbol{\xi}) \boldsymbol{\zeta}$ is in the form

$$
\boldsymbol{\zeta}^{\top} \boldsymbol{C}(\boldsymbol{\xi}) \boldsymbol{\zeta}=\left(\boldsymbol{\zeta}^{\top} \mathrm{C}_{1}(\boldsymbol{\xi}) \boldsymbol{\zeta}, \ldots, \boldsymbol{\zeta}^{\top} \mathrm{C}_{n-1}(\boldsymbol{\xi}) \boldsymbol{\zeta}\right)
$$

where $\mathbf{C}_{i}$ are $2 \pi$-periodic matrices. It follows directly from (48B), (39B), and expression (3.3) for the Jacobi matrix $\boldsymbol{\theta}^{\prime}$ that

$$
\begin{array}{r}
\boldsymbol{\chi}=\left(\mathbf{I}_{n-1}+\mathbf{u}^{\prime}\right)^{-1} \delta \mathbf{u}, \\
\boldsymbol{\lambda}=\mathbf{W}^{-1} \delta \mathbf{W}-\chi_{i} \mathbf{W}^{-1} \frac{\partial}{\partial \xi_{i}} \mathbf{W}, \\
\boldsymbol{\mu}=\mathbf{V}^{-1}\left(\delta \mathbf{v}-\chi_{i} \frac{\partial}{\partial \xi_{i}} \mathbf{v}-\boldsymbol{\Lambda} \boldsymbol{\lambda}\right), \\
\boldsymbol{\Gamma}=\mathbf{W}^{-1} \delta \mathbf{w}-\chi_{i} \mathbf{W}^{-1} \frac{\partial}{\partial \xi_{i}} \mathbf{W} .
\end{array}
$$

Hence it remains to prove that

$$
\begin{gathered}
\mathbf{A}=-\nabla \boldsymbol{\chi}, \quad \mathbf{B}=\nabla(\mathbf{J} \boldsymbol{\lambda}) \\
\mathbf{C}_{i}=\frac{\partial}{\partial \xi_{i}}(\mathbf{J} \boldsymbol{\Gamma}), \quad \mathbf{J} \boldsymbol{\Gamma}+\boldsymbol{\Gamma}^{\top} \mathbf{J}=0, \\
\boldsymbol{\mu}=\boldsymbol{\nu}+\nabla \psi_{0} .
\end{gathered}
$$

To this end notice that $\boldsymbol{\theta}^{\prime \top} \mathbf{J}_{2 n} \boldsymbol{\theta}^{\prime}=\mathbf{J}_{2 n}$, which yields

$$
(\delta \boldsymbol{\theta})^{\prime \top} \mathbf{J}_{2 n} \boldsymbol{\theta}^{\prime}+\boldsymbol{\theta}^{\prime \top} \mathbf{J}_{2 n}(\delta \boldsymbol{\theta})^{\prime}=0 .
$$

Now set $\boldsymbol{\sigma}=(\boldsymbol{\xi}, \boldsymbol{\eta}, \boldsymbol{\zeta}) \in R^{2 n}$ and notice that the vector-valued function $\boldsymbol{\pi}$ takes it value in $\mathbb{R}^{2 n}$. Denote by $\pi_{k}$ the components of $\boldsymbol{\pi}$. Differentiation of the equality $\delta \boldsymbol{\theta}=\boldsymbol{\theta}^{\prime} \boldsymbol{\pi}$ leads to the identity

$$
\delta \boldsymbol{\theta}^{\prime}=\boldsymbol{\theta}^{\prime} \boldsymbol{\pi}^{\prime}+\pi_{k} \frac{\partial}{\partial \sigma_{k}} \boldsymbol{\theta}^{\prime} .
$$

Substituting this result in $(10 \mathrm{~B})$ we arrive at

$$
\boldsymbol{\pi}^{\prime \top} \boldsymbol{\theta}^{\prime \top} \mathbf{J}_{2 n} \boldsymbol{\theta}^{\prime}+\boldsymbol{\theta}^{\prime \top} \mathbf{J}_{2 n} \boldsymbol{\theta}^{\prime} \boldsymbol{\pi}^{\prime}+\pi_{k}\left\{\boldsymbol{\theta}^{\prime \top} \mathbf{J}_{2 n} \frac{\partial}{\partial \sigma_{k}} \boldsymbol{\theta}^{\prime}+\frac{\partial}{\partial \sigma_{k}} \boldsymbol{\theta}^{\prime \top} \mathbf{J}_{2 n} \boldsymbol{\theta}^{\prime}\right\} .=0 .
$$


Notice that

$$
\boldsymbol{\theta}^{\prime \top} \mathbf{J}_{2 n} \frac{\partial}{\partial \sigma_{k}} \boldsymbol{\theta}^{\prime}+\frac{\partial}{\partial \sigma_{k}} \boldsymbol{\theta}^{\prime \top} \mathbf{J}_{2 n} \boldsymbol{\theta}^{\prime}=\frac{\partial}{\partial \sigma_{k}}\left(\boldsymbol{\theta}^{\prime \top} \mathbf{J}_{2 n} \boldsymbol{\theta}^{\prime}\right) \equiv \frac{\partial}{\partial \sigma_{k}} \mathbf{J}_{2 n}=0
$$

Recalling that $\boldsymbol{\theta}^{\prime \top} \mathbf{J}_{2 n} \boldsymbol{\theta}^{\prime}=\mathbf{J}_{2 n}$ we obtain

$$
\boldsymbol{\pi}^{\prime \top} \mathbf{J}_{2 n}+\mathbf{J}_{2 n} \boldsymbol{\pi}^{\prime}=0 .
$$

It follows from (48B) that

$$
\boldsymbol{\pi}^{\prime}=\left(\begin{array}{ccc}
P_{11}, & 0, & 0 \\
P_{21}, & P_{22}, & P_{23} \\
P_{31}, & 0, & P_{33}
\end{array}\right), \quad \mathbf{J}_{2 n}=\left(\begin{array}{ccc}
0 & \mathbf{I}_{n-1} & 0 \\
-\mathbf{I}_{n-1} & 0 & 0 \\
0 & 0 & \mathbf{J}
\end{array}\right)
$$

where

$$
\begin{gathered}
P_{11}=\chi^{\prime}, \quad P_{21}\left(\boldsymbol{\mu}+\mathbf{A} \boldsymbol{\eta}+\mathbf{B} \boldsymbol{\zeta}+\frac{1}{2} \boldsymbol{\zeta}^{\top} \boldsymbol{C} \boldsymbol{\zeta}\right)_{\xi}^{\prime} \\
P_{22}=\mathbf{A} \quad P_{23}=\mathbf{B}+\mathbf{C} \boldsymbol{\zeta}, \\
P_{31}=\lambda_{\xi}^{\prime}+(\boldsymbol{\Gamma} \boldsymbol{\zeta})_{\xi}^{\prime}, \quad P_{33}=\boldsymbol{\Gamma},
\end{gathered}
$$

Here $\mathbf{C} \boldsymbol{\lambda}$ is $(n-1) \times 2$ matrix with the entries $(\mathbf{C} \boldsymbol{\lambda})_{i j}=C_{i, j p} \lambda_{p}$. Substituting $(56 \mathrm{~B})$ into (55B) we obtain four nontrivial matrix equations

$$
\begin{gathered}
P_{22}=-P_{11}^{\top}, \quad P_{23}=\left(\mathbf{J} P_{31}^{\top},\right. \\
P_{33}^{\top} \mathbf{J}+\mathbf{J} P_{33}=0, \quad P_{21}=P_{21}^{\top} .
\end{gathered}
$$

The first equation gives $\mathbf{A}=-\nabla \boldsymbol{\chi}$. The second gives two nontrivial relation

$$
\mathbf{B}=(\mathbf{J} \boldsymbol{\lambda})_{\xi}^{\prime \top} \equiv \nabla(\mathbf{J} \boldsymbol{\lambda}), \quad \mathbf{C} \boldsymbol{\zeta}=\nabla_{\xi}(\mathbf{J} \boldsymbol{\Gamma} \boldsymbol{\zeta})
$$

The latter equality is equivalent to relations $\mathbf{C}_{i}=\partial(\mathbf{J} \boldsymbol{\Gamma}) / \partial \xi_{i}$. Finally notice that relation $P_{21}=P_{21}^{\top}$ gives $\boldsymbol{\mu}_{\xi}^{\prime}=\left(\boldsymbol{\mu}_{\xi}^{\prime}\right)^{\top}$. In other words, the Jacobi matrix of the vector-field $\boldsymbol{\mu}$ is symmetric. It follows that the vector field $\boldsymbol{\mu}$ is potential. Hence it has the representation $\boldsymbol{\mu}=\boldsymbol{\nu}+\nabla \psi_{0}$, where $\boldsymbol{\nu}$ is a constant vector and $\psi_{0}$ is $2 \pi$-periodic function with zero mean. Therefore the matrices $\mathbf{A}, \mathbf{B}, \boldsymbol{\Gamma}$ and the vector field $\boldsymbol{\mu}$ satisfy conditions (51B)-(53B). This completes the proof of Theorem 3.2 . 


\section{Proof of Lemma 4.1}

Equation (4.9b) along with the equality $\mathbf{V}^{-1}=\nabla(\boldsymbol{\xi}+\mathbf{u})$ imply

$$
\boldsymbol{\omega}^{\top} \nabla(\boldsymbol{\xi}+\mathbf{u})=\nabla_{y} H_{m}(\boldsymbol{\xi}+\mathbf{u}, \mathbf{v}, \mathbf{w}),
$$

which yields the first relation in (4.11). Next, recalling formula (4.9f) for $\boldsymbol{\Lambda}$ , we obtain

$$
\left(\frac{\partial H_{m}}{\partial \mathbf{y}}(\boldsymbol{\xi}+\mathbf{u}, \mathbf{v}, \mathbf{w}) \boldsymbol{\Lambda}\right)_{q}=-\frac{\partial H_{m}}{\partial y_{i}}(\boldsymbol{\xi}+\mathbf{u}, \mathbf{v}, \mathbf{w}) V_{i j} \frac{\partial w_{k}}{\partial \xi_{j}} J_{k p} W_{p q},
$$

We also have

$$
\left(\frac{\partial H_{m}}{\partial \mathbf{z}}(\boldsymbol{\xi}+\mathbf{u}, \mathbf{v}, \mathbf{w}) W\right)_{q}=\frac{\partial H_{m}}{\partial z_{p}}(\boldsymbol{\xi}+\mathbf{u}, \mathbf{v}, \mathbf{w}) W_{p q}
$$

From this and (4.9c) we obtain

$$
\left\{-\frac{\partial H_{m}}{\partial y_{i}}(\boldsymbol{\xi}+\mathbf{u}, \mathbf{v}, \mathbf{w}) V_{i j} \frac{\partial w_{k}}{\partial \xi_{j}} J_{k p}+\frac{\partial H_{m}}{\partial z_{p}}(\boldsymbol{\xi}+\mathbf{u}, \mathbf{v}, \mathbf{w})\right\} W_{p q}=0 .
$$

Notice that in view of (4.9b), we have $\partial H_{m} / \partial y_{i} V_{i j}=\omega_{j}$. Thus we get

$$
\left\{-\boldsymbol{\partial} \mathbf{w}^{\top} \mathbf{J}+\frac{\partial H_{m}}{\partial \mathbf{z}}\right\} \mathbf{W}=0
$$

which yields the third inequality in (4.11). In order to derive the second equality in (4.11) we differentiate both sides of (4.9a) with respect to $x_{i}$ to obtain

$$
\frac{\partial H_{m}}{\partial x_{i}}(\xi+\mathbf{u}, \mathbf{v}, \mathbf{w})+\frac{\partial H_{m}}{\partial y_{n}}(\xi+\mathbf{u}, \mathbf{v}, \mathbf{w}) \frac{\partial v_{n}}{\partial x_{i}}+\frac{\partial H_{m}}{\partial z_{p}}(\xi+\mathbf{u}, \mathbf{v}, \mathbf{w}) \frac{\partial w_{p}}{\partial x_{i}}=0
$$

Since $\mathbf{V}=\left(\mathbf{I}+\mathbf{u}^{\prime}\right)^{-\top}$, we have $\nabla_{x}=\mathbf{V} \nabla_{\xi}$. It follows that

$$
\frac{\partial}{\partial y_{n}} H_{m}(\xi+\mathbf{u}, \mathbf{v}, \mathbf{w}) \frac{\partial}{\partial x_{n}}=\frac{\partial}{\partial y_{n}} H_{m}(\xi+\mathbf{u}, \mathbf{v}, \mathbf{w}) V_{n j} \frac{\partial}{\partial \xi_{j}}
$$

From this and (4.9b) we obtain

$$
\frac{\partial}{\partial y_{n}} H_{m}(\xi+\mathbf{u}, \mathbf{v}, \mathbf{w}) \frac{\partial}{\partial x_{n}}=\omega_{i} \frac{\partial}{\partial \xi_{i}}=\boldsymbol{\partial}
$$


On the other hand, relation (4.9f) implies $\mathbf{v}=\boldsymbol{\beta}+\nabla_{x} \varphi_{0}-w_{1} \nabla_{x} w_{2}$. Thus we get

$$
\frac{\partial v_{n}}{\partial x_{i}}=\frac{\partial^{2} \varphi_{0}}{\partial x_{n} \partial x_{i}}-w_{1} \frac{\partial^{2} w_{2}}{\partial x_{n} \partial x_{i}}-\frac{\partial w_{1}}{\partial x_{n}} \frac{\partial w_{2}}{\partial x_{i}} .
$$

Combining this identity with $(2 \mathrm{C})$ we arrive at

$$
\begin{array}{r}
\frac{\partial}{\partial y_{n}} H_{m}(\xi+\mathbf{u}, \mathbf{v}, \mathbf{w}) \frac{\partial v_{n}}{\partial x_{i}}=\boldsymbol{\partial} \frac{\partial \varphi_{0}}{\partial x_{i}} \\
-w_{2} \boldsymbol{\partial} \frac{\partial w_{1}}{\partial x_{i}}-\boldsymbol{\partial} w_{1} \frac{\partial w_{2}}{\partial x_{i}}= \\
\boldsymbol{\partial} v_{i}-\boldsymbol{\partial} w_{1} \frac{\partial w_{2}}{\partial x_{i}}+\boldsymbol{\partial} w_{2} \frac{\partial w_{1}}{\partial x_{i}}
\end{array}
$$

From this and the latter relation in (4.11) we obtain

$$
\begin{array}{r}
\frac{\partial H_{m}}{\partial y_{n}}(\xi+\mathbf{u}, \mathbf{v}, \mathbf{w}) \frac{\partial v_{n}}{\partial x_{i}}= \\
\boldsymbol{\partial} v_{i}-\frac{\partial H_{m}}{\partial z_{2}} \frac{\partial w_{2}}{\partial x_{i}}-\frac{\partial H_{m}}{\partial z_{1}} \frac{\partial w_{1}}{\partial x_{i}}
\end{array}
$$

Substituting this equality into (1C) we obtain the second equality in (4.11).

\section{Proof of Theorem 4.7}

Direct calculations lead to the following formulae

$$
\begin{gathered}
\delta \Phi_{1}=\frac{\partial H_{m}}{\partial \mathbf{x}}(\boldsymbol{\xi}+\mathbf{u}, \mathbf{v}, \mathbf{w}) \delta \mathbf{u}+ \\
\frac{\partial H_{m}}{\partial \mathbf{y}}(\boldsymbol{\xi}+\mathbf{u}, \mathbf{v}, \mathbf{w}) \delta \mathbf{v}+\frac{\partial H_{m}}{\partial \mathbf{z}}(\boldsymbol{\xi}+\mathbf{u}, \mathbf{v}, \mathbf{w}) \delta \mathbf{w} \\
\delta \Phi_{2}=\left\{\frac{\partial H_{m}}{\partial \mathbf{y}}(\boldsymbol{\xi}+\mathbf{u}, \mathbf{v}, \mathbf{w}) \delta \boldsymbol{\Lambda}+\frac{\partial H_{m}}{\partial \mathbf{z}}(\boldsymbol{\xi}+\mathbf{u}, \mathbf{v}, \mathbf{w}) \delta \mathbf{W}\right\}^{\top}+ \\
\left\{(\delta \mathbf{u})^{\top}\left(\frac{\partial^{2} H_{m}}{\partial \mathbf{x} \partial \mathbf{y}}(\boldsymbol{\xi}+\mathbf{u}, \mathbf{v}, \mathbf{w}) \boldsymbol{\Lambda}+\frac{\partial^{2} H_{m}}{\partial \mathbf{x} \partial \mathbf{z}}(\boldsymbol{\xi}+\mathbf{u}, \mathbf{v}, \mathbf{w}) \mathbf{W}\right)\right\}^{\top}+ \\
\left\{(\delta \mathbf{v})^{\top}\left(\frac{\partial^{2} H_{m}}{\partial y^{2}}(\boldsymbol{\xi}+\mathbf{u}, \mathbf{v}, \mathbf{w}) \boldsymbol{\Lambda}+\frac{\partial^{2} H_{m}}{\partial \mathbf{y} \partial \mathbf{z}}(\boldsymbol{\xi}+\mathbf{u}, \mathbf{v}, \mathbf{w}) \mathbf{W}\right)\right\}^{\top}+ \\
\left\{(\delta \mathbf{w})^{\top}\left(\frac{\partial^{2} H_{m}}{\partial \mathbf{z} \partial \mathbf{y}}(\boldsymbol{\xi}+\mathbf{u}, \mathbf{v}, \mathbf{w}) \boldsymbol{\Lambda}+\frac{\partial^{2} H_{m}}{\partial \mathbf{z}^{2}}(\boldsymbol{\xi}+\mathbf{u}, \mathbf{v}, \mathbf{w}) \mathbf{W}\right)\right\}^{\top},
\end{gathered}
$$




$$
\begin{gathered}
\delta \Phi_{3}=\left\{\frac{\partial H_{m}}{\partial \mathbf{y}}(\boldsymbol{\xi}+\mathbf{u}, \mathbf{v}, \mathbf{w}) \delta \mathbf{V}+(\delta \mathbf{u})^{\top} \frac{\partial^{2} H_{m}}{\partial \mathbf{x} \partial \mathbf{y}}(\boldsymbol{\xi}+\mathbf{u}, \mathbf{v}, \mathbf{w})\right\}^{\top}+ \\
\left\{(\delta \mathbf{v})^{\top} \frac{\partial^{2} H_{m}}{\partial \mathbf{y}^{2}}(\boldsymbol{\xi}+\mathbf{u}, \mathbf{v}, \mathbf{w}) \mathbf{V}+(\delta \mathbf{w})^{\top} \frac{\partial^{2} H_{m}}{\partial \mathbf{z} \partial \mathbf{y}}(\boldsymbol{\xi}+\mathbf{u}, \mathbf{v}, \mathbf{w}) \mathbf{V}\right\}^{\top} \\
\delta \Phi_{4}=\frac{\partial H_{m}}{\partial y_{i}}(\boldsymbol{\xi}+\mathbf{u}, \mathbf{v}, \mathbf{w}) \delta \mathbf{R}_{i}+(\mathbf{A}+\mathbf{B}+\mathbf{C})+(\mathbf{A}+\mathbf{B}+\mathbf{C})^{\top}+ \\
(\delta \mathbf{u})^{\top} \mathbf{P}^{(x)}+(\delta \mathbf{v})^{\top} \mathbf{P}^{(y)}+(\delta \mathbf{w})^{\top} \mathbf{P}^{(z)} \\
\delta \Phi_{5}=\frac{1}{(2 \pi)^{n-1}} \int_{\mathbb{T}^{n-1}} \delta w_{1} d \boldsymbol{\xi}
\end{gathered}
$$

where

$$
\begin{array}{r}
\mathbf{A}=\delta \boldsymbol{\Lambda}^{\top} \frac{\partial^{2} H_{m}}{\partial \mathbf{y}^{2}}(\boldsymbol{\xi}+\mathbf{u}, \mathbf{v}, \mathbf{w}) \boldsymbol{\Lambda}, \\
\mathbf{B}=\delta \mathbf{W}^{\top} \frac{\partial^{2} H_{m}}{\partial \mathbf{z} \partial \mathbf{y}}(\boldsymbol{\xi}+\mathbf{u}, \mathbf{v}, \mathbf{w}) \boldsymbol{\Lambda}+\mathbf{W}^{\top} \frac{\partial^{2} H_{m}}{\partial \mathbf{z} \partial \mathbf{y}}(\boldsymbol{\xi}+\mathbf{u}, \mathbf{v}, \mathbf{w}) \delta \boldsymbol{\Lambda}, \\
\mathbf{C}=\delta \mathbf{W}^{\top} \frac{\partial^{2} H_{m}}{\partial \mathbf{z}^{2}}(\boldsymbol{\xi}+\mathbf{u}, \mathbf{v}, \mathbf{w}) \mathbf{W}, \\
\mathbf{P}^{(\tau)}=\boldsymbol{\Lambda}^{\top} \frac{\partial^{3} H_{m}}{\partial \boldsymbol{\tau} \partial \mathbf{y}^{2}}(\boldsymbol{\xi}+\mathbf{u}, \mathbf{v}, \mathbf{w}) \boldsymbol{\Lambda}+\boldsymbol{\Lambda}^{\top} \frac{\partial^{3} H_{m}}{\partial \boldsymbol{\tau} \partial \mathbf{y} \partial \mathbf{z}}(\boldsymbol{\xi}+\mathbf{u}, \mathbf{v}, \mathbf{w}) \mathbf{W}+ \\
\mathbf{W}^{\top} \frac{\partial^{3} H_{m}}{\partial \tau \partial \mathbf{z} \partial \mathbf{y}}(\boldsymbol{\xi}+\mathbf{u}, \mathbf{v}, \mathbf{w}) \boldsymbol{\Lambda}+\mathbf{W}^{\top} \frac{\partial^{3} H_{m}}{\partial \boldsymbol{\tau} \partial \mathbf{z}^{2}}(\boldsymbol{\xi}+\mathbf{u}, \mathbf{v}, \mathbf{w}) \mathbf{W},
\end{array}
$$

where $\boldsymbol{\tau}=\mathbf{x}, \mathbf{y}, \mathbf{z}$. The variations $\delta \mathbf{V}, \delta \mathbf{W}, \delta \boldsymbol{\Lambda}$, and $\delta \mathbf{v}$ are defined in terms of the components of $\delta \boldsymbol{\varphi}$ by relations (3.13).

In view of the Second Structure Theorem (3.2) the mapping (4.22) has the inverse given by formulae (3.19). In particular, we have

$$
\begin{gathered}
\delta \mathbf{u}=\chi_{i} \frac{\partial}{\partial \xi_{i}}(\boldsymbol{\xi}+\mathbf{u}), \quad \delta \mathbf{v}=\mathbf{V} \boldsymbol{\mu}+\boldsymbol{\Lambda} \boldsymbol{\lambda}+\chi_{i} \frac{\partial}{\partial \xi_{i}} \mathbf{v} \\
\delta \mathbf{w}=\mathbf{W} \boldsymbol{\lambda}+\chi_{i} \frac{\partial}{\partial \xi_{i}} \mathbf{w}
\end{gathered}
$$

Substituting these equalities in representation (1Da) for $\delta \Phi_{1}$ we arrive at

$$
\begin{array}{r}
\delta \Phi_{1}=\frac{\partial H_{m}}{\partial \mathbf{y}} \mathbf{V} \boldsymbol{\mu}+\left(\frac{\partial H_{m}}{\partial \mathbf{x}} \frac{\partial}{\partial \xi_{i}}(\mathrm{Id}+\mathbf{u})+\frac{\partial H_{m}}{\partial \mathbf{y}} \frac{\partial \mathbf{v}}{\partial \xi_{i}}+\frac{\partial H_{m}}{\partial \mathbf{z}} \frac{\partial \mathbf{w}}{\partial \xi_{i}}\right) \chi_{i}+ \\
\left(\frac{\partial H_{m}}{\partial \mathbf{y}} \boldsymbol{\Lambda}+\frac{\partial H_{m}}{\partial \mathbf{z}} \mathbf{W}\right) \boldsymbol{\lambda}
\end{array}
$$


Here the derivatives of $H_{m}$ are calculated at the point $(\boldsymbol{\xi}+\mathbf{u}, \mathbf{v}, \mathbf{w})$. It follows from formula (4.9a) for $\Phi_{1}$ that

$$
\frac{\partial H_{m}}{\partial \mathbf{x}} \frac{\partial}{\partial \xi_{i}}(\mathrm{Id}+\mathbf{u})+\frac{\partial H_{m}}{\partial \mathbf{y}} \frac{\partial \mathbf{v}}{\partial \xi_{i}}+\frac{\partial H_{m}}{\partial \mathbf{z}} \frac{\partial \mathbf{w}}{\partial \xi_{i}}=\frac{\partial \Phi_{1}}{\partial \xi_{i}}
$$

Next, formula (4.9c) for $\Phi_{3}$ and the equality $\boldsymbol{\mu}=\nabla \psi_{0}+\delta \boldsymbol{\beta}$ imply

$$
\frac{\partial H_{m}}{\partial \mathbf{y}} \mathbf{V} \boldsymbol{\mu}=\boldsymbol{\omega}^{\top} \boldsymbol{\mu}+\left(\frac{\partial H_{m}}{\partial \mathbf{y}} \mathbf{V}-\boldsymbol{\omega}^{\top}\right) \boldsymbol{\mu}=\boldsymbol{\partial} \psi_{0}+\boldsymbol{\omega}^{\top} \cdot \delta \boldsymbol{\beta}+\Phi_{3}^{\top} \boldsymbol{\mu} .
$$

Formula (4.9b) for $\Phi_{2}$ yields

$$
\left(\frac{\partial H_{m}}{\partial \mathbf{y}} \boldsymbol{\Lambda}+\frac{\partial H_{m}}{\partial \mathbf{z}} \mathbf{W}\right) \lambda=\Phi_{2}^{\top} \boldsymbol{\lambda} .
$$

Substituting (4D)-(6D) into (3D) we obtain the desired representation (4.23a) for $\delta \Phi_{1}$. Let us turn to the representation for $\delta \Phi_{2}$. Equalities (3.19d), (3.19f) in the second structural theorem imply

$$
\begin{gathered}
\delta \boldsymbol{\Lambda}=\mathbf{V} \nabla_{\xi}(\mathbf{J} \boldsymbol{\lambda})+\chi_{i} \frac{\partial}{\partial \xi_{i}} \boldsymbol{\Lambda}+\boldsymbol{\lambda}^{\top} \mathbf{R}+\boldsymbol{\Lambda} \boldsymbol{\Gamma}, \\
\delta \mathbf{W}=\mathbf{W} \boldsymbol{\Gamma}+\chi_{i} \frac{\partial}{\partial \xi_{i}} \mathbf{W} .
\end{gathered}
$$

Substituting these relations along with equalities (2D) into (1Db) we obtain

$$
\begin{aligned}
& \delta \Phi_{2}^{\top}=\frac{\partial H_{m}}{\partial \mathbf{y}} \mathbf{V} \nabla_{\xi}(\mathbf{J} \boldsymbol{\lambda})+\frac{\partial H_{m}}{\partial \mathbf{y}}\left(\boldsymbol{\lambda}^{\top} \mathbf{R}\right)+ \\
& \chi_{i} \frac{\partial H_{m}}{\partial \mathbf{y}} \frac{\partial \boldsymbol{\Lambda}}{\partial \xi_{i}}+\frac{\partial H_{m}}{\partial \mathbf{y}} \boldsymbol{\Lambda} \boldsymbol{\Gamma}+\chi_{i} \frac{\partial H_{m}}{\partial \mathbf{z}} \frac{\partial \mathbf{W}}{\partial \xi_{i}}+\frac{\partial H_{m}}{\partial \mathbf{z}} \mathbf{W} \boldsymbol{\Gamma}+ \\
& \chi_{i} \frac{\partial}{\partial \xi_{i}}(\boldsymbol{\xi}+\mathbf{u})^{\top}\left(\frac{\partial^{2} H_{m}}{\partial \boldsymbol{x} \partial \boldsymbol{y}} \boldsymbol{\Lambda}+\frac{\partial^{2} H_{m}}{\partial \boldsymbol{x} \partial \boldsymbol{z}} \mathbf{W}\right)+ \\
& \chi_{i} \frac{\partial \mathbf{v}^{\top}}{\partial \xi_{i}}\left(\frac{\partial^{2} H_{m}}{\partial \boldsymbol{y}^{2}} \boldsymbol{\Lambda}+\frac{\partial^{2} H_{m}}{\partial \boldsymbol{y} \partial \boldsymbol{z}} \mathbf{W}\right)+\chi_{i} \frac{\partial \mathbf{w}^{\top}}{\partial \xi_{i}}\left(\frac{\partial^{2} H_{m}}{\partial \boldsymbol{z} \partial \boldsymbol{y}} \boldsymbol{\Lambda}+\frac{\partial^{2} H_{m}}{\partial \boldsymbol{z}^{2}} \mathbf{W}\right)+ \\
& \boldsymbol{\lambda}^{\top} \boldsymbol{\Lambda}^{\top}\left(\frac{\partial^{2} H_{m}}{\partial \boldsymbol{y}^{2}} \boldsymbol{\Lambda}+\frac{\partial^{2} H_{m}}{\partial \boldsymbol{y} \partial \boldsymbol{z}} \mathbf{W}\right)+\boldsymbol{\lambda}^{\top} \mathbf{W}^{\top}\left(\frac{\partial^{2} H_{m}}{\partial \boldsymbol{z} \partial \boldsymbol{y}} \boldsymbol{\Lambda}+\frac{\partial^{2} H_{m}}{\partial \boldsymbol{z}^{2}} \mathbf{W}\right)+ \\
& \boldsymbol{\mu}^{\top} \mathbf{V}^{\top}\left(\frac{\partial^{2} H_{m}}{\partial \boldsymbol{y}^{2}} \boldsymbol{\Lambda}+\frac{\partial^{2} H_{m}}{\partial \boldsymbol{y} \partial \boldsymbol{z}} \mathbf{W}\right)
\end{aligned}
$$


Since $\partial H_{m} / \partial \mathbf{y}$ is the row vector with the components $\partial H_{m} / \partial y_{i}$, we have

$$
\frac{\partial H_{m}}{\partial \mathbf{y}}\left(\boldsymbol{\lambda}^{\top} \mathbf{R}\right)=\boldsymbol{\lambda}^{\top}\left(\frac{\partial H_{m}}{\partial y_{i}} \mathbf{R}_{i}\right) .
$$

Recall that the matrices $\mathbf{R}_{i}$ and $\Phi_{4}$ are symmetric. From this and formula (4.9d), we conclude that

$$
\begin{gathered}
\frac{\partial H_{m}}{\partial \mathbf{y}}\left(\boldsymbol{\lambda}^{\top} \mathbf{R}\right)+\boldsymbol{\lambda}^{\top} \boldsymbol{\Lambda}^{\top}\left(\frac{\partial^{2} H_{m}}{\partial \boldsymbol{y}^{2}} \boldsymbol{\Lambda}+\frac{\partial^{2} H_{m}}{\partial \boldsymbol{y} \partial \boldsymbol{z}} \mathbf{W}\right) \\
+\boldsymbol{\lambda}^{\top} \mathbf{W}^{\top}\left(\frac{\partial^{2} H_{m}}{\partial \boldsymbol{z} \partial \boldsymbol{y}} \boldsymbol{\Lambda}+\frac{\partial^{2} H_{m}}{\partial \boldsymbol{z}^{2}} \mathbf{W}\right)=\boldsymbol{\lambda}^{\top} \Phi_{4}^{\top}
\end{gathered}
$$

Next, it follows from (4.9b) that

$$
\begin{aligned}
& \frac{\partial H_{m}}{\partial \mathbf{y}} \frac{\partial \boldsymbol{\Lambda}}{\partial \xi_{i}}+\frac{\partial H_{m}}{\partial \mathbf{z}} \frac{\partial \mathbf{W}}{\partial \xi_{i}}+\frac{\partial}{\partial \xi_{i}}(\boldsymbol{\xi}+\mathbf{u})^{\top}\left(\frac{\partial^{2} H_{m}}{\partial \boldsymbol{x} \partial \boldsymbol{y}} \boldsymbol{\Lambda}+\frac{\partial^{2} H_{m}}{\partial \boldsymbol{x} \partial \boldsymbol{z}} \mathbf{W}\right)+ \\
& \frac{\partial \mathbf{v}^{\top}}{\partial \xi_{i}}\left(\frac{\partial^{2} H_{m}}{\partial \boldsymbol{y}^{2}} \boldsymbol{\Lambda}+\frac{\partial^{2} H_{m}}{\partial \boldsymbol{y} \partial \boldsymbol{z}} \mathbf{W}\right)+\frac{\partial \mathbf{w}^{\top}}{\partial \xi_{i}}\left(\frac{\partial^{2} H_{m}}{\partial \boldsymbol{z} \partial \boldsymbol{y}}+\frac{\partial^{2} H_{m}}{\partial \boldsymbol{z}^{2}} \mathbf{W}\right)=\frac{\partial \Phi_{2}^{\top}}{\partial \xi_{i}} .
\end{aligned}
$$

We also have

$$
\frac{\partial H_{m}}{\partial \mathbf{y}} \boldsymbol{\Lambda} \boldsymbol{\Gamma}+\frac{\partial H_{m}}{\partial \mathbf{z}} \mathbf{W} \boldsymbol{\Gamma}=\Phi_{2}^{\top} \boldsymbol{\Gamma} .
$$

Substituting (9D)-(11D) into (8D) we obtain

$$
\begin{array}{r}
\delta \Phi_{2}^{\top}=\frac{\partial H_{m}}{\partial \mathbf{y}} \mathbf{V} \nabla_{\xi}(\mathbf{J} \boldsymbol{\lambda})+\boldsymbol{\mu}^{\top} \mathbf{V}^{\top}\left(\frac{\partial^{2} H_{m}}{\partial \boldsymbol{y}^{2}} \boldsymbol{\Lambda}+\frac{\partial^{2} H_{m}}{\partial \boldsymbol{y} \partial \boldsymbol{z}} \mathbf{W}\right)+ \\
\chi_{i} \frac{\partial \Phi_{2}^{\top}}{\partial \xi_{i}}+\Phi_{2}^{\top} \boldsymbol{\Gamma}+\boldsymbol{\lambda}^{\top} \Phi_{4}^{\top}
\end{array}
$$

Expression (4.25) for the matrix $\mathbf{T}$ implies

$$
\boldsymbol{\mu}^{\top} \mathbf{V}^{\top}\left(\frac{\partial^{2} H_{m}}{\partial \boldsymbol{y}^{2}} \boldsymbol{\Lambda}+\frac{\partial^{2} H_{m}}{\partial \boldsymbol{y} \partial \boldsymbol{z}} \mathbf{W}\right)=\boldsymbol{\mu}^{\top} \boldsymbol{T}^{\top}
$$

On the other hand, formula (4.9c) for the operator $\Phi_{3}$ yields

$$
\begin{array}{r}
\frac{\partial H_{m}}{\partial \mathbf{y}} \mathbf{V} \nabla_{\xi}(\mathbf{J} \boldsymbol{\lambda})=\boldsymbol{\omega}^{\top} \nabla_{\xi}(\mathbf{J} \boldsymbol{\lambda})+\Phi_{3}^{\top} \nabla_{\xi}(\mathbf{J} \boldsymbol{\lambda})= \\
(\boldsymbol{\partial}(\mathbf{J} \boldsymbol{\lambda}))^{\top}+\left((\mathbf{J} \boldsymbol{\lambda})_{\xi}^{\prime} \Phi_{3}\right)^{\top}=(\mathbf{J} \boldsymbol{\partial} \boldsymbol{\lambda})^{\top}+\left((\mathbf{J} \boldsymbol{\lambda})_{\xi}^{\prime} \Phi_{3}\right)^{\top} .
\end{array}
$$


Substituting this identity along with (13D) into (12D) we arrive at desired representation (4.23b) for $\delta \Phi_{2}$.

Our next task is to prove identity (4.23c) for $\delta \Phi_{3}$. It follows from identity (3.19e) in the Second Structural Theorem that

$$
\delta \mathbf{V}=-\mathbf{V} \nabla_{\xi} \boldsymbol{\chi}+\chi_{i} \frac{\partial \mathbf{V}}{\partial \xi_{i}}
$$

Substituting this identity along with (2D) into formula (1Dc) for $\delta \Phi_{3}$ we obtain

$$
\begin{gathered}
\delta \Phi_{3}^{\top}=-\frac{\partial H_{m}}{\partial \mathbf{y}} \mathbf{V} \nabla_{\xi} \boldsymbol{\chi}+\chi_{i} \frac{\partial H_{m}}{\partial \mathbf{y}} \frac{\partial \mathbf{V}}{\partial \xi_{i}}+ \\
\chi_{i} \frac{\partial}{\partial \xi_{i}}(\boldsymbol{\xi}+\mathbf{u})^{\top} \frac{\partial^{2} H_{m}}{\partial \mathbf{x} \partial \mathbf{y}}+\chi_{i} \frac{\partial \mathbf{v}^{\top}}{\partial \xi_{i}} \frac{\partial^{2} H_{m}}{\partial \mathbf{y}^{2}}+\chi_{i} \frac{\partial \mathbf{w}^{\top}}{\partial \xi_{i}} \frac{\partial^{2} H_{m}}{\partial \mathbf{z} \partial \mathbf{y}}+ \\
\boldsymbol{\mu}^{\top} \mathbf{V}^{\top} \frac{\partial^{2} H_{m}}{\partial \mathbf{y}^{2}} \mathbf{V}+\boldsymbol{\lambda}^{\top} \boldsymbol{\Lambda}^{\top} \frac{\partial^{2} H_{m}}{\partial \mathbf{y}^{2}} \mathbf{V}+\boldsymbol{\lambda}^{\top} \mathbf{W}^{\top} \frac{\partial^{2} H_{m}}{\partial \mathbf{z} \partial \mathbf{y}} \mathbf{V}
\end{gathered}
$$

It follows from formula (4.9c) for $\Phi_{3}$ that

$$
-\frac{\partial H_{m}}{\partial \mathbf{y}} \mathbf{V} \nabla_{\xi} \boldsymbol{\chi}=-\boldsymbol{\omega}^{\top} \nabla_{\xi} \boldsymbol{\chi}-\Phi_{3}^{\top} \nabla_{\xi} \boldsymbol{\chi}=-(\boldsymbol{\partial} \chi)^{\top}-\left(\boldsymbol{\chi}_{\xi}^{\prime} \Phi_{3}\right)^{\top}
$$

Next, we have

$$
\begin{gathered}
\chi_{i} \frac{\partial H_{m}}{\partial \mathbf{y}} \frac{\partial \mathbf{V}}{\partial \xi_{i}}+\chi_{i} \frac{\partial}{\partial \xi_{i}}(\boldsymbol{\xi}+\mathbf{u})^{\top} \frac{\partial^{2} H_{m}}{\partial \mathbf{x} \partial \mathbf{y}}+ \\
\chi_{i} \frac{\partial \mathbf{v}^{\top}}{\partial \xi_{i}} \frac{\partial^{2} H_{m}}{\partial \mathbf{y}^{2}}+\chi_{i} \frac{\partial \mathbf{w}^{\top}}{\partial \xi_{i}} \frac{\partial^{2} H_{m}}{\partial \mathbf{z} \partial \mathbf{y}}=\chi_{i} \frac{\partial \Phi_{3}^{\top}}{\partial \xi_{i}}
\end{gathered}
$$

On the other hand, formulae (4.25) for $\mathbf{S}$ and $\mathbf{T}$ yield

$$
\boldsymbol{\mu}^{\top} \mathbf{V}^{\top} \frac{\partial^{2} H_{m}}{\partial \mathbf{y}^{2}} \mathbf{V}+\boldsymbol{\lambda}^{\top} \boldsymbol{\Lambda}^{\top} \frac{\partial^{2} H_{m}}{\partial \mathbf{y}^{2}} \mathbf{V}+\boldsymbol{\lambda}^{\top} \mathbf{W}^{\top} \frac{\partial^{2} H_{m}}{\partial \mathbf{z} \partial \mathbf{y}} \mathbf{V}=(\mathbf{S} \boldsymbol{\mu})^{\top}+\left(\mathbf{T}^{\top} \boldsymbol{\lambda}\right)^{\top} .
$$

Substituting (15D)-(17D) into (14D) we arrive at the desired identity (1Dc).

It remains to prove representation (1Dd) for $\delta \Phi_{4}$. Recall that this relation includes the matrices $\mathbf{A}, \mathbf{B}, \mathbf{C}$, given by (1Df), and the matrices $\mathbf{P}^{(\tau)}$, $\boldsymbol{\tau}=\mathbf{x}, \mathbf{y}, \mathbf{z}$ given by (1Dg). Substituting representations (7D) into (1Df) we obtain

$$
\mathbf{A}=\boldsymbol{\Lambda}^{\top} \frac{\partial^{2} H_{m}}{\partial \mathbf{y}^{2}} \boldsymbol{\Lambda}+\chi_{i} \frac{\partial \boldsymbol{\Lambda}^{\top}}{\partial \xi_{i}} \frac{\partial^{2} H_{m}}{\partial \mathbf{y}^{2}} \boldsymbol{\Lambda}+(\mathbf{J} \boldsymbol{\lambda})_{\xi}^{\prime} \mathbf{V}^{\top} \frac{\partial^{2} H_{m}}{\partial \mathbf{y}^{2}} \boldsymbol{\Lambda}+\left(\boldsymbol{\lambda}^{\top} \mathbf{R}\right)^{\top} \frac{\partial^{2} H_{m}}{\partial \mathbf{y}^{2}} \boldsymbol{\Lambda}
$$




$$
\begin{gathered}
\mathbf{B}=\chi_{i} \frac{\partial \mathbf{W}^{\top}}{\partial \xi_{i}} \frac{\partial^{2} H_{m}}{\partial \mathbf{z y}} \boldsymbol{\Lambda}+\boldsymbol{\Gamma}^{\top} \frac{\partial^{2} H_{m}}{\partial \mathbf{z y}} \boldsymbol{\Lambda}+ \\
\mathbf{W}^{\top} \frac{\partial^{2} H_{m}}{\partial \mathbf{z y}} \mathbf{V} \nabla_{\xi}(\mathbf{J} \boldsymbol{\lambda})+\chi_{i} \mathbf{W}^{\top} \frac{\partial^{2} H_{m}}{\partial \mathbf{z y}} \frac{\partial \boldsymbol{\Lambda}}{\partial \xi_{i}}+\mathbf{W}^{\top} \frac{\partial^{2} H_{m}}{\partial \mathbf{z y}}\left(\boldsymbol{\lambda}^{\top} \mathbf{R}\right)+\mathbf{W}^{\top} \frac{\partial^{2} H_{m}}{\partial \mathbf{z y}} \boldsymbol{\Lambda} \boldsymbol{\Gamma} \\
\mathbf{C}=\chi_{i} \frac{\partial \mathbf{W}^{\top}}{\partial \xi_{i}} \frac{\partial^{2} H_{m}}{\partial \mathbf{z y}} \mathbf{W}+\boldsymbol{\Gamma}^{\top} W^{\top} \frac{\partial^{2} H_{m}}{\partial \mathbf{z y}} \mathbf{W}
\end{gathered}
$$

Next, expression (1Dg) for $\mathbf{P}^{(\tau)}$ and representations (2D) implies

$$
\begin{gathered}
\delta \mathbf{u}^{\top} \mathbf{P}^{(x)}+\delta \mathbf{v}^{\top} \mathbf{P}^{(y)}+\delta \mathbf{w}^{\top} \mathbf{P}^{(z)}=\chi_{i}\left(\frac{\partial \mathbf{u}^{\top}}{\partial \xi_{i}} \mathbf{P}^{(x)}+\frac{\partial \mathbf{v}^{\top}}{\partial \xi_{i}} \mathbf{P}^{(y)}+\frac{\partial \mathbf{w}^{\top}}{\partial \xi_{i}} \mathbf{P}^{(z)}\right)+ \\
\boldsymbol{\mu}^{\top} \mathbf{P}^{(y)}+\boldsymbol{\lambda}^{\top}\left(\boldsymbol{\Lambda}^{\top} \mathbf{P}^{(y)}+\mathbf{W}^{\top} \mathbf{P}^{(z)}\right)
\end{gathered}
$$

Substituting (18D) (21D) into (1Dd) and recalling the representation

$$
\delta \mathbf{R}_{i}=\frac{\partial}{\partial \xi_{i}}(\mathbf{J} \boldsymbol{\Gamma})+\mathbf{R}_{i} \boldsymbol{\Gamma}+\left(\mathbf{R}_{i} \boldsymbol{\Gamma}\right)^{\top}+\chi_{i} \frac{\partial}{\partial \xi_{i}} \mathbf{R}_{i}
$$

in the second structural Theorem 3.2 we arrive at the identity

$$
\begin{gathered}
\delta \Phi_{4}=\frac{\partial H_{m}}{\partial y_{i}} V_{i k} \frac{\partial}{\partial \xi_{k}}(\mathbf{J} \boldsymbol{\Gamma})+\mathbf{Q} \boldsymbol{\Gamma}+\boldsymbol{\Gamma}^{\top} \mathbf{Q}^{\top}+ \\
\chi_{k} \mathbf{N}_{k}+\mathbf{U}+\mathbf{U}^{\top}+\mathbf{K}+\mathbf{K}^{\top}+\boldsymbol{\Sigma} .
\end{gathered}
$$

Here

$$
\begin{aligned}
& \mathbf{Q}=\frac{\partial H_{m}}{\partial y_{i}} \mathbf{R}_{i}+\Lambda^{\top} \frac{\partial^{2} H_{m}}{\partial \mathbf{y}^{2}} \boldsymbol{\Lambda}+ \\
& \mathbf{W}^{\top} \frac{\partial^{2} H_{m}}{\partial \mathbf{z} \partial \mathbf{y}} \boldsymbol{\Lambda}+\left(\mathbf{W}^{\top} \frac{\partial^{2} H_{m}}{\partial \mathbf{z} \partial \mathbf{y}} \boldsymbol{\Lambda}\right)^{\top}+\mathbf{W}^{\top} \frac{\partial^{2} H_{m}}{\partial \mathbf{z}^{2}} \mathbf{W}=\boldsymbol{\Omega}+\Phi_{4}, \\
& \mathbf{N}_{k}=\frac{\partial H_{m}}{\partial y_{i}} \frac{\partial \mathbf{R}_{i}}{\partial \xi_{k}}+\boldsymbol{\Lambda}^{\top} \frac{\partial^{2} H_{m}}{\partial \mathbf{y}^{2}} \frac{\partial \boldsymbol{\Lambda}}{\partial \xi_{k}}+\frac{\partial \boldsymbol{\Lambda}^{\top}}{\partial \xi_{k}} \frac{\partial^{2} H_{m}}{\partial \mathbf{y}^{2}} \boldsymbol{\Lambda}+ \\
& \mathbf{W}^{\top} \frac{\partial^{2} H_{m}}{\mathbf{z} \partial \mathbf{y}} \frac{\partial \boldsymbol{\Lambda}}{\partial \xi_{k}}+\frac{\partial \mathbf{W}^{\top}}{\partial \xi_{k}} \frac{\partial^{2} H_{m}}{\partial \mathbf{z} \partial \mathbf{y}} \boldsymbol{\Lambda}+ \\
& \left(\mathbf{W}^{\top} \frac{\partial^{2} H_{m}}{\partial \mathbf{z} \partial \mathbf{y}} \frac{\partial \boldsymbol{\Lambda}}{\partial \xi_{k}}+\frac{\partial \mathbf{W}^{\top}}{\partial \xi_{k}} \frac{\partial^{2} H_{m}}{\partial \mathbf{z y}} \boldsymbol{\Lambda}\right)^{\top}+ \\
& \mathbf{W}^{\top} \frac{\partial^{2} H_{m}}{\partial \mathbf{z}^{2}} \frac{\partial \mathbf{W}}{\partial \xi_{k}}+\frac{\partial \mathbf{W}^{\top}}{\partial \xi_{k}} \frac{\partial^{2} H_{m}}{\partial \mathbf{z}^{2}} \mathbf{W}+ \\
& +\frac{\partial \mathbf{u}^{\top}}{\partial \xi_{i}} \mathbf{P}^{(x)}+\frac{\partial \mathbf{v}^{\top}}{\partial \xi_{i}} \mathbf{P}^{(y)}+\frac{\partial \mathbf{w}^{\top}}{\partial \xi_{i}} \mathbf{P}^{(z)}=\frac{\partial \Phi_{4}}{\partial \xi_{k}} .
\end{aligned}
$$


Next notice that in view of (4.9c),

$$
\frac{\partial}{\partial \xi_{i}}(\mathbf{J} \boldsymbol{\Gamma})=\boldsymbol{\partial}(\mathbf{J} \boldsymbol{\Gamma})+\Phi_{3, k} \frac{\partial}{\partial \xi_{k}}(\mathbf{J} \boldsymbol{\Gamma})
$$

Substituting this relation along with (4.23), (4.25) into (4.22) we arrive at the identity

$$
\begin{gathered}
\delta \Phi_{4}=\boldsymbol{\partial}(\mathbf{J} \boldsymbol{\Gamma})+\boldsymbol{\Omega} \boldsymbol{\Gamma}+\boldsymbol{\Gamma}^{\top} \boldsymbol{\Omega}^{\top}+ \\
\Phi_{4} \boldsymbol{\Gamma}+\boldsymbol{\Gamma}^{\top} \Phi_{4}^{\top}+\chi_{k} \frac{\partial \Phi_{4}}{\partial \xi_{k}}+\Phi_{3, k} \frac{\partial}{\partial \xi_{k}}(\mathbf{J} \boldsymbol{\Gamma})+ \\
\mathbf{U}+\mathbf{U}^{\top}+\mathbf{K}+\mathbf{K}^{\top}+\boldsymbol{\Sigma} .
\end{gathered}
$$

Notice that $\mathbf{U}$ is the matrix-valued linear form of $\partial \lambda_{i} / \partial \xi_{j}$. Hence it admits the representation

$$
\mathbf{U}+\mathbf{U}^{\top}=\frac{\partial \lambda_{i}}{\partial \xi_{j}} \mathbf{U}_{i j}
$$

where $\mathbf{U}_{i j}$ are symmetric matrix-valued functions. Next, $\mathbf{K}$ and $\boldsymbol{\Sigma}$ are matrix-valued linear form of $\lambda_{i}$ and $\mu_{j}$. Hence there are symmetric matrixvalued functions $\mathbf{E}_{i}$ and $\mathbf{K}_{i}$ such that

$$
\mathbf{K}+\mathbf{K}^{\top}+\boldsymbol{\Sigma}=\mu_{i} \boldsymbol{E}_{i}+\lambda_{i} \mathbf{K}_{i}
$$

Substituting (4.27) and (4.28) into (4.26) we obtain the desired representation (4.24d).

\section{E Proof of Theorems 5.1 and 8.1}

\section{E.1 Proof of Theorem 5.1}

This section is devoted to the proof of solvability of the following problem

$$
\begin{gathered}
\boldsymbol{\partial} \psi_{0}+\delta q+\delta p \cdot\left(w_{1}-\alpha\right)+\frac{1}{2} \delta M\left(w_{1}-\alpha\right)^{2}=F_{1}, \\
\mathbf{J} \boldsymbol{\partial} \boldsymbol{\lambda}+\mathbf{T} \boldsymbol{\mu}+\mathbf{W}^{\top} \delta \mathbf{p}+\delta M\left(w_{1}-\alpha\right) \mathbf{W}^{\top} \mathbf{e}_{1}=F_{2}, \quad \boldsymbol{\mu}=\nabla \psi_{0}+\delta \boldsymbol{\beta} \\
-\boldsymbol{\partial} \boldsymbol{\chi}+\mathbf{S} \boldsymbol{\mu}+\mathbf{T}^{\top} \boldsymbol{\lambda}=F_{3}, \\
\boldsymbol{\partial}(\mathbf{J} \boldsymbol{\Gamma})+\boldsymbol{\Omega} \boldsymbol{\Gamma}+(\boldsymbol{\Omega} \boldsymbol{\Gamma})^{\top}+\mathbf{U}_{i j} \frac{\partial \lambda_{i}}{\partial \xi_{j}}+\mu_{i} \mathbf{E}_{i}+\lambda_{i} \mathbf{K}_{i}+ \\
+\mathbf{W}^{\top} \delta \mathbf{M W}=F_{4}, \quad \Gamma_{11}=-\Gamma_{22},
\end{gathered}
$$




$$
\begin{gathered}
\frac{1}{(2 \pi)^{n-1}} \int_{\mathbb{T}^{n-1}}\left[\psi_{0}-\delta \boldsymbol{\beta} \cdot \mathbf{u}+w_{2}(\mathbf{W} \boldsymbol{\lambda})_{1}\right] d \xi=f_{1} \\
\frac{1}{(2 \pi)^{n-1}} \int_{\mathbb{T}^{n-1}} \mathbf{V}^{-\top} \chi d \xi=f_{3}, \quad \frac{1}{(2 \pi)^{n-1}} \int_{\mathbb{T}^{n-1}}(\mathbf{W} \boldsymbol{\Gamma})_{12} d \xi=f_{4} \\
\frac{1}{(2 \pi)^{n-1}} \int_{\mathbb{T}^{n-1}}\left((\mathbf{W} \boldsymbol{\lambda})_{1}+\boldsymbol{\chi} \cdot \nabla w_{1}\right) d \boldsymbol{\xi}=f_{5}
\end{gathered}
$$

The following theorem constitutes the existence and uniqueness of solutions to problem (1E)

Theorem E.1. Let a fixed $\sigma \in[1 / 2,1], d \geq 2$, and the matrix $\mathbf{K}_{0}=\mathbf{S}_{0}-$ $\mathbf{t}_{0} \otimes \mathbf{t}_{0}$ given by (2.11), satisfies the condition det $\mathbf{K}_{0} \neq 0$. Furthermore, assume that

$$
\left\|w_{1}-\alpha\right\|_{\sigma, 0}+\|\mathbf{W}-\mathbf{I}\|_{\sigma_{0}}+\left\|\mathbf{u}^{\prime}-\mathbf{I}\right\|_{\sigma, 0} \leq c r
$$

and

$$
\begin{gathered}
\left\|\mathbf{S}-\mathbf{S}_{0}\right\|_{\sigma, 0}+\left\|\mathbf{T}-\mathbf{T}_{0}\right\|_{\sigma, 0} \leq c(r+|\varepsilon|), \\
\left\|\mathbf{U}_{i j}\right\|_{\sigma, 0}+\left\|\mathbf{E}_{i}\right\|_{\sigma, 0}+\left\|\mathbf{K}_{i}\right\|_{\sigma, 0} \leq c .
\end{gathered}
$$

Then there are $\varepsilon_{0}>0$ and $r_{0}>0$ with the following properties. For every

$$
\begin{gathered}
r \leq r_{0} \quad|\varepsilon| \leq \varepsilon_{0}, \\
0 \leq \sigma_{0}<\sigma_{1}<\sigma, \quad 1 / 4 \geq \sigma_{1}, \sigma \in[1 / 4,1)
\end{gathered}
$$

and for all

$\mathbf{F}=\left(F_{1}, F_{2}, F_{3}, F_{4}, f_{1}, f_{3}, f_{4}, f_{5}\right) \in \mathcal{A}_{\sigma_{1}, 0} \times \mathcal{A}_{\sigma_{1}, 0}^{2} \times \mathcal{A}_{\sigma_{1}, 0}^{n-1} \times \mathcal{A}_{\sigma_{1}, 0}^{4} \times \mathbb{C} \times \mathbb{C}^{n-1} \mathbb{C} \times \mathbb{C}$, with $F_{4}=F_{4}^{\top}$, problem $(\underline{1 \mathrm{E}})$ has a unique solution

$$
\left(\psi_{0}, \boldsymbol{\lambda}, \boldsymbol{\chi}, \boldsymbol{\Gamma}, \delta \boldsymbol{\beta}, q, p, \delta M\right) \in \mathcal{A}_{\sigma_{0}, 0} \times \mathcal{A}_{\sigma_{0}, 0}^{2} \times \mathcal{A}_{\sigma, 0}^{n-1} \times \mathcal{A}_{\sigma_{0}, 0}^{4} \times \mathbb{C}^{n-1} \times \mathbb{C}^{3} .
$$

This solution admits the estimate

$$
\left\|\left(\psi_{0}, \boldsymbol{\lambda}, \boldsymbol{\chi}, \boldsymbol{\Gamma}\right)\right\|_{\sigma_{0}, 0}+|(\delta \boldsymbol{\beta}, q, p, \delta M)| \leq c\left(\sigma_{1}-\sigma_{0}\right)^{-8 n-12}\|\mathbf{F}\|_{\sigma_{1}, 0}
$$

where the constant $c$ is independent of $\varepsilon_{0}, r_{0}$, and $\sigma_{i}$. 
Notice that identity $\mathbf{V}=\left(\mathbf{I}+\mathbf{u}^{\prime}\right)^{-\top}$ implies that

$$
\left\|\mathbf{V}^{-\top}-\mathbf{I}\right\|_{\sigma_{0}} \leq c r .
$$

The rest of the section is devoted to the proof of this theorem. Our strategy is the following. First we prove the existence and uniqueness of solutions to a triangle truncated problem. Then we prove the solvability of equations (5.6) using the contraction mapping principle. Our considerations are based on the following existence and uniqueness results for model differential equations with constant coefficients

Equations with constant coefficients Recall the denotations

$$
\overline{\boldsymbol{\mu}}=\frac{1}{(2 \pi)^{n-1}} \int_{\mathbb{T}^{n-1}} \boldsymbol{\mu}(\boldsymbol{\xi}) d \boldsymbol{\xi}, \quad \boldsymbol{\mu}^{*}=\boldsymbol{\mu}-\overline{\boldsymbol{\mu}}
$$

Lemma E.2. For every $g \in \mathcal{A}_{\sigma_{1}, 0}$ with $\bar{g}=0$, the equations

$$
\partial \psi_{0}=g, \quad \bar{\psi}_{0}=0
$$

have a unique solution $\psi_{0} \in \mathcal{A}_{\sigma_{0}, 0}$ such that

$$
\left\|\psi_{0}\right\|_{\sigma_{0}, 0} \leq c\left(\sigma_{1}-\sigma_{0}\right)^{-\tau}\|g\|_{\sigma_{1}, 0}, \quad\left|\psi_{0}\right|_{s} \leq c|g|_{s+\tau}
$$

where $\tau=n+1$ and $0 \leq \sigma_{0}<\sigma_{1}$ are arbitrary numbers.

Proof. Substituting the decomposition

$$
\psi_{0}(\xi)=\sum_{\mathbf{s} \in \mathbb{Z}^{n-1} \backslash\{0\}} \widehat{\psi_{0}}(\mathbf{s}) e^{i \mathbf{s} \boldsymbol{\xi}}, \quad \widehat{\psi_{0}}(\mathbf{s})=(2 \pi)^{\frac{1-n}{2}} \int_{\mathbb{T}^{n-1}} \psi_{0}(\boldsymbol{\xi}) e^{-i \mathbf{s} \boldsymbol{\xi}} d \boldsymbol{\xi}
$$

into equation (65Ea) we can rewrite this equation into the equivalent form

$$
\widehat{\psi_{0}}(\mathbf{s})=(i \boldsymbol{\omega} \cdot \mathbf{s})^{-1} \widehat{g}(\mathbf{s}) \text { for } \mathbf{s} \in \mathbb{Z}^{n-1} \backslash\{0\}, \quad \widehat{\psi_{0}}(0)=0
$$

The diophantine condition implies

$$
\left|(\boldsymbol{\omega} \cdot \mathbf{s})^{-1}\right| \leq c|\mathbf{s}|^{-n}
$$

which gives

$$
\left|\widehat{\psi_{0}}(\mathbf{s})\right| \leq(1+|\mathbf{s}|)^{n}|\widehat{g}(\mathbf{s})|
$$


It is well-known that for every $\sigma \in[0,1)$ and $\sigma^{\prime} \in(0, \sigma)$, and for every measurable function $g: \mathbb{T}^{n-1} \rightarrow \mathbb{C}$, we have

$$
\sup _{\mathbf{s} \in \mathbb{Z}^{n-1}} e^{\sigma|\mathbf{s}|}|\widehat{g}(\mathbf{s})| \leq\|g\|_{\sigma, 0}, \quad\|g\|_{\sigma^{\prime}, 0} \leq \frac{c}{\sigma-\sigma^{\prime}} \sup _{\mathbf{s} \in \mathbb{Z}^{n-1}} e^{\sigma|\mathbf{s}|}|\widehat{g}(\mathbf{s})|
$$

where $c$ is independent of $g, \sigma$, and $\sigma^{\prime}$. ¿From this and (10E) that

$$
\begin{array}{r}
e^{\left(\sigma_{0}+\sigma_{1}\right)|\mathbf{s}| / 2}\left|\widehat{\psi_{0}}(\mathbf{s})\right| \leq c(1+|\mathbf{s}|)^{n} e^{\left(\sigma_{0}+\sigma_{1}\right)|\mathbf{s}| / 2}|| \widehat{g}(\mathbf{s}) \mid \leq \\
c\left(\sigma_{1}-\sigma_{0}\right)^{-n} e^{\sigma_{1}|\mathbf{s}|}|| \widehat{g}(\mathbf{s}) \mid \leq c\left(\sigma_{1}-\sigma_{0}\right)^{-\tau}\|g\|_{\sigma_{1}, 0}
\end{array}
$$

It follows that

$$
\left\|\psi_{0}\right\|_{\sigma_{0}, 0} \leq \frac{c}{\sigma_{1}-\sigma_{0}} \sup _{\mathbf{s} \in \mathbb{Z}^{n-1}} e^{\left(\sigma_{0}+\sigma_{1}\right)|\mathbf{s}| / 2}\left|\widehat{\psi_{0}}(\mathbf{s})\right| \leq c\left(\sigma_{1}-\sigma_{0}\right)^{-\tau}\|g\|_{\sigma_{1}, 0}
$$

Thus we get the first estimate in (7E). The second obviously follows from (10E) and the definition of the Sobolev space $H_{r}$.

Lemma E.3. Let $0 \leq \sigma_{0}<\sigma_{1}$. Let $\mathbf{H} \in \mathcal{A}_{\sigma_{1}, 0}$ satisfies the condition $\overline{\mathbf{H}}=0$. Then the equation

$$
\mathbf{J} \partial \boldsymbol{\lambda}+\boldsymbol{\Omega} \boldsymbol{\lambda}=\mathbf{H}
$$

has a unique solution $\boldsymbol{\lambda} \in \mathcal{A}_{\sigma_{0}, 0}$, satisfying the condition $\overline{\boldsymbol{\lambda}}=0$. This solution admits the estimates

$$
\begin{gathered}
\|\boldsymbol{\lambda}\|_{\sigma_{0}, 0} \leq c\left(\sigma_{1}-\sigma_{0}\right)^{-2 n-3}\|\mathbf{H}\|_{\sigma_{1}, 0} . \\
|\boldsymbol{\lambda}|_{s} \leq c|\mathbf{H}|_{s+2 n+3} .
\end{gathered}
$$

Proof. Equation (12E) is equivalent to the following system of the linear algebraic equations for the Fourier coefficients $\widehat{\lambda}(\mathbf{s}), \mathbf{s} \in \mathbb{Z}^{n-1}$, of $\boldsymbol{\lambda}$,

$$
i(\boldsymbol{\omega} \cdot \mathbf{s}) \mathbf{J} \widehat{\boldsymbol{\lambda}}(\mathbf{s})+\boldsymbol{\Omega} \widehat{\boldsymbol{\lambda}}(\mathbf{s})=\widehat{\mathbf{H}}(\mathbf{s}), \quad \mathbf{s} \in \mathbb{Z}^{n-1} \backslash\{0\}, \quad \widehat{\boldsymbol{\lambda}}(0)=0 .
$$

This equality along with expressions (2.6) and (2.9) for the matrices $\mathbf{J}$ and $\Omega$ implies

$\widehat{\lambda_{1}}(\mathbf{s})=-\left(k+(\boldsymbol{\omega} \cdot \mathbf{s})^{2}\right)^{-1}\left(\widehat{H_{1}}(\mathbf{s})-i(\boldsymbol{\omega} \cdot \mathbf{s}) \widehat{H_{2}}(\mathbf{s})\right), \widehat{\lambda_{1}}(\mathbf{s})=\widehat{H_{2}}(\mathbf{s})+i(\boldsymbol{\omega} \cdot \mathbf{s}) \widehat{\lambda_{1}}(\mathbf{s})$.

Recall that $\operatorname{Re} k \geq 0$. From this and the diophantine estimate (9E) we conclude that

$$
|\widehat{\boldsymbol{\lambda}}(\mathbf{s})| \leq c|\mathbf{s}|^{2 n+2}|\widehat{\mathbf{H}}(\mathbf{s})|, \quad \mathbf{s} \in \mathbb{Z}^{n-1}
$$


Thus we get

$$
\begin{gathered}
\|\boldsymbol{\lambda}\|_{\sigma_{0}, 0} \leq c\left(\sigma_{1}-\sigma_{0}\right)^{-1} \sup _{\mathbf{s} \in \mathbb{Z}^{n-1}} e^{\left(\sigma_{0}+\sigma_{1}\right)|\mathbf{s}| / 2}|\widehat{\boldsymbol{\lambda}}(\mathbf{s})| \\
c\left(\sigma_{1}-\sigma_{0}\right)^{-2 n-3} \sup _{\mathbf{s} \in \mathbb{Z}^{n-1}} e^{\left(\sigma_{1}|\mathbf{s}|\right.}|\widehat{\mathbf{H}}(\mathbf{s})| \leq c\left(\sigma_{1}-\sigma_{0}\right)^{-2 n-3}\|\mathbf{H}\|_{\sigma_{1}, 0},
\end{gathered}
$$

and the lemma follows.

Now we consider the matrix differential equation

$$
\begin{gathered}
\boldsymbol{\partial}(\mathbf{J} \boldsymbol{\Gamma})+\boldsymbol{\Omega} \boldsymbol{\Gamma}+(\boldsymbol{\Omega} \boldsymbol{\Gamma})^{\top}+\delta \mathbf{M}=\mathbf{G}, \\
\Gamma_{11}=-\Gamma_{22}, \quad \int_{\mathbb{T}^{n-1}} \Gamma_{12} d \xi=f,
\end{gathered}
$$

Her $\delta \mathbf{M}=\delta M \operatorname{diag}(1,0)$ is the unknown matrix.

Lemma E.4. For all $(\mathbf{G}, f) \in A_{\sigma_{1}, 0} \times \mathbb{C}$ and for all $0 \leq \sigma_{0}<\sigma_{1}$, equations (15E)-(16E) have a unique solution $(\boldsymbol{\Gamma}, \delta M) \in \mathcal{A}_{\sigma_{0}, 0}^{4} \times \mathbb{C}$. This solution admits the estimate

$$
\|\boldsymbol{\Gamma}\|_{\sigma_{0}, 0}+|\delta \mathbf{M}| \leq c\left(\sigma_{1}-\sigma_{0}\right)^{-3 n-2}\left(\|\mathbf{G}\|_{\sigma_{1}, 0}+|f|\right) .
$$

Proof. Rewrite equations (15E)-(16E) in the form

$$
\begin{array}{r}
\Omega \bar{\Gamma}+(\Omega \overline{\boldsymbol{\Gamma}})^{\top}+\delta \mathbf{M}=\overline{\mathbf{G}}, \\
\bar{\Gamma}_{11}=-\bar{\Gamma}_{22}, \quad \bar{\Gamma}_{12}=f_{4}, \quad \delta \mathbf{M}=\operatorname{diag}(\delta M, 0) . \\
\boldsymbol{\partial}\left(\mathbf{J} \Gamma^{*}\right)+\boldsymbol{\Omega} \Gamma^{*}+\left(\Omega \Gamma^{*}\right)^{\top}=\mathbf{G}^{*}, \quad \Gamma_{11}^{*}=-\Gamma_{22}^{*} .
\end{array}
$$

The unique solution to equations (18E) is given by

$$
\overline{\boldsymbol{\Gamma}}=\left(\begin{array}{cc}
-\bar{G}_{22} / 2, & f_{4} \\
k f_{4}+\bar{G}_{21} & \bar{G}_{22} / 2
\end{array}\right), \quad \delta \mathbf{M}=\left(\begin{array}{cc}
\bar{G}_{11}-k \bar{G}_{22}, & 0 \\
0 & 0
\end{array}\right) .
$$

It follows that

$$
|\overline{\boldsymbol{\Gamma}}|+|\delta M| \leq c|\mathbf{G}| .
$$

Next, using the Fourier transform we can write equation (19E) in the form of the matrix equation

$i(\boldsymbol{\omega} \cdot \boldsymbol{s}) \widehat{\mathbf{J} \boldsymbol{\Gamma}^{*}}(\mathbf{s})+\boldsymbol{\Omega} \widehat{\Gamma^{*}}(\mathbf{s})+\left(\widehat{\Omega} \widehat{\Gamma^{*}}(\mathbf{s})\right)^{\top}=\widehat{\boldsymbol{G}^{*}}(\mathbf{s}), \quad \widehat{\Gamma_{11}^{*}}(\mathbf{s})=-\widehat{\Gamma_{22}^{*}}(\mathbf{s})$ for $\mathbf{s} \in \mathbb{Z}^{2} \backslash\{0\}$, 
Recall that

$$
\widehat{\boldsymbol{\Gamma}^{*}}(0)=\widehat{\boldsymbol{G}}^{*}(0)=0 .
$$

In it turn, this equation is equivalent to the system of linear algebraic equations

$$
\begin{gathered}
i(\boldsymbol{\omega} \cdot \mathbf{s}) \widehat{\Gamma_{21}^{*}}(\mathbf{s})+2 k \widehat{\Gamma_{22}^{*}}(\mathbf{s})=\widehat{G_{11}^{*}}(\mathbf{s}), \\
i(\boldsymbol{\omega} \cdot \mathbf{s}) \widehat{\Gamma_{22}^{*}}(\mathbf{s})+\widehat{\Gamma_{21}^{*}}-k \widehat{\Gamma_{12}^{*}}(\mathbf{s})=\widehat{G_{12}^{*}}(\mathbf{s}), \\
-i(\boldsymbol{\omega} \cdot \mathbf{s}) \widehat{\Gamma_{12}^{*}}(\mathbf{s})+2 \widehat{\Gamma_{22}^{*}}(\mathbf{s})=\widehat{G_{22}^{*}}(\mathbf{s}), \\
\widehat{\Gamma_{11}^{*}}(\mathbf{s})=-\widehat{\Gamma_{22}^{*}}(\mathbf{s}) \text { for } \mathbf{s} \in \mathbb{Z}^{2} \backslash\{0\} .
\end{gathered}
$$

Straightforward calculations give

$\widehat{\Gamma_{12}^{*}}(\mathbf{s})=\frac{2}{(\boldsymbol{\omega} \cdot \mathbf{s})^{2}+4 k}\left(\frac{1}{i(\boldsymbol{\omega} \cdot \mathbf{s})}\left(\widehat{G_{11}^{*}}(\mathbf{s})-k \widehat{G_{22}^{*}}(\mathbf{s})\right)+i(\boldsymbol{\omega} \cdot \mathbf{s}) \widehat{G_{22}^{*}}(\mathbf{s})-\widehat{G_{12}^{*}}(\mathbf{s})\right)$

and

$$
\begin{gathered}
\widehat{\Gamma_{22}^{*}}(\mathbf{s})=\frac{1}{2} \widehat{G_{22}^{*}}(\mathbf{s})+\frac{1}{2} i(\boldsymbol{\omega} \cdot \mathbf{s}) \widehat{\Gamma_{12}^{*}}(\mathbf{s}), \\
\widehat{\Gamma_{21}^{*}}(\mathbf{s})=\widehat{G_{12}^{*}}(\mathbf{s})+k \widehat{\Gamma_{12}^{*}}(\mathbf{s})-i(\boldsymbol{\omega} \cdot \mathbf{s}) \widehat{\Gamma_{22}^{*}}(\mathbf{s}) \\
\widehat{\Gamma_{11}^{*}}(\mathbf{s})=-\widehat{\Gamma_{22}^{*}}(\mathbf{s}) .
\end{gathered}
$$

These identities and the diophantine estimate (9E) imply the estimate

$$
\left|\widehat{\Gamma^{*}}(\mathbf{s})\right| \leq c|\mathbf{s}|^{3 n+1}\left|\widehat{\mathbf{G}^{*}}(\mathbf{s})\right| \text { for } \mathbf{s} \in \mathbb{Z}^{2} .
$$

Set $\varsigma=\left(\sigma_{1}-\sigma_{0}\right) / 3$. Arguing as in the proof of Lemmas E.3 and 17E we obtain

$$
\begin{array}{r}
\left\|\boldsymbol{\Gamma}^{*}\right\|_{\sigma_{0}, 0} \leq c \varsigma^{-1} \sup _{\mathbf{s}} e^{\left(\sigma_{0}+\varsigma\right)|\mathbf{s}|}\left|\widehat{\boldsymbol{\Gamma}^{*}}(\mathbf{s})\right| \leq \\
c \varsigma^{-1} \sup _{\mathbf{s}}|\mathbf{s}|^{3 n+1} e^{\left(\sigma_{0}+\varsigma\right)|\mathbf{s}|}\left|\widehat{G^{*}}(\mathbf{s})\right| \leq c \varsigma^{-3 n-2} \sup _{\mathbf{s}} e^{\left(\sigma_{0}+\varsigma\right)|\mathbf{s}|}\left|\widehat{G^{*}}(\mathbf{s})\right| \\
\leq c \varsigma^{-3 n-2}\left\|\mathbf{G}^{*}\right\|_{\sigma_{0}+\varsigma, 0} .
\end{array}
$$

Combining this estimate with (20E) we arrive at

$$
\|\boldsymbol{\Gamma}\|_{\sigma_{0}, 0}+|\delta \mathbf{M}| \leq c \varsigma^{-3 n-2}\|\boldsymbol{G}\|_{\sigma_{0}+\varsigma, 0} .
$$

This completes the proof of the lemma. 
Truncated equations. Our next task is to prove the existence and uniqueness of analytic solutions to linear equations with variable coefficients which can be regarded as simplification of the main problem (1E). First we consider the shortened version of this problem which can be formulated as follows. It is necessary to find periodic functions $\psi_{0}, \boldsymbol{\lambda}, \boldsymbol{\chi}$ and parameters $p, q$ and $\beta$ satisfying the following equations.

$$
\begin{gathered}
\boldsymbol{\partial} \psi_{0}+q=F_{1}, \\
\mathbf{J} \boldsymbol{\partial} \boldsymbol{\lambda}+\boldsymbol{\Omega} \boldsymbol{\lambda}+\mathbf{T} \boldsymbol{\mu}+p \mathbf{e}_{1}=F_{2}, \quad \boldsymbol{\mu}=\delta \boldsymbol{\beta}+\nabla \psi_{0} \\
-\boldsymbol{\partial} \boldsymbol{\chi}+\mathbf{S} \boldsymbol{\mu}+\mathbf{T}^{\top} \boldsymbol{\lambda}=F_{3}, \\
\overline{\psi_{0}}=f_{1}, \quad \overline{\boldsymbol{\chi}}=f_{3}, \quad \overline{\lambda_{1}}=f_{5} .
\end{gathered}
$$

Proposition E.5. Under the assumptions of Theorem E.1, there exist $r_{0}>$ $0, \varepsilon_{0}>0$, and $c>0$ such that for all $r \leq r_{0},|\varepsilon| \leq \varepsilon_{0}$, and for all $\left(f_{5}, f_{3}, f_{1}\right) \in$ $\mathbb{C}^{n+1}, F_{i} \in \mathcal{A}_{\sigma_{1}, 0}$, and for all $0 \leq \sigma_{0}<\sigma_{1}$, equations (23E) have a unique solution analytic solution. This solution admits the estimate

$$
\begin{aligned}
\left\|\psi_{0}\right\|_{\sigma_{0}, 0}+\|\boldsymbol{\lambda}\|_{\sigma_{0}, 0}+\|\boldsymbol{\chi}\|_{\sigma_{0}, 0}+ \\
|p|+|q|+|\delta \boldsymbol{\beta}| \leq c\left(\sigma_{1}-\sigma_{0}\right)^{-6 n-8}\left(\|\mathbf{F}\|_{\sigma_{1}, 0}+|\mathbf{f}|\right) .
\end{aligned}
$$

Proof. We have

$$
\boldsymbol{\lambda}=\overline{\boldsymbol{\lambda}}+\boldsymbol{\lambda}^{*}, \quad \boldsymbol{\chi}=f_{3}+\boldsymbol{\chi}^{*}
$$

Substituting this decomposition into (23E) we obtain two system of equations

$$
\begin{gathered}
\overline{\psi_{0}}=f_{1}, \quad \bar{\chi}=f_{3}, \quad \overline{\lambda_{1}}=f_{5} \\
\boldsymbol{\Omega} \overline{\boldsymbol{\lambda}}+\overline{\mathbf{T}} \boldsymbol{\beta}+p \mathbf{e}_{1}=\overline{F_{2}}-\overline{\mathbf{T} \nabla \psi_{0}}, \\
\overline{\mathbf{S}} \boldsymbol{\beta}+\overline{\mathbf{T}}^{\top} \overline{\boldsymbol{\lambda}}=\overline{F_{3}}-\overline{\mathbf{S} \nabla \psi_{0}}-\overline{\mathbf{T}^{\top} \boldsymbol{\lambda}^{*},}
\end{gathered}
$$

and

$$
\begin{gathered}
\boldsymbol{\partial} \psi_{0}^{*}=F_{1}^{*}, \\
\mathbf{J} \boldsymbol{\partial} \boldsymbol{\lambda}^{*}+\boldsymbol{\Omega} \boldsymbol{\lambda}^{*}+\left(\mathbf{T} \nabla \psi_{0}^{*}\right)^{*}=F_{2}^{*}-\mathbf{T}^{*} \boldsymbol{\beta}, \\
-\boldsymbol{\partial} \boldsymbol{\chi}^{*}+\left(\mathbf{S} \nabla \psi_{0}^{*}\right)^{*}+\left(\mathbf{T}^{\top} \boldsymbol{\lambda}^{*}\right)=F_{3}^{*}-\mathbf{S}^{*} \boldsymbol{\beta}-\left(\mathbf{T}^{\top}\right)^{*} \bar{\lambda},
\end{gathered}
$$

The rest of the proof is based on the following auxiliary lemma. 
Lemma E.6. . Under the assumptions of Theorem 1 E there exist $r_{0}>0$ and $\varepsilon_{0}>0, c>0$ such that for all $r \leq r_{0}$ and $|\varepsilon| \leq \varepsilon_{0}$, and for given $\nabla \psi_{0}, \lambda^{*} \in H_{0}$, the system (26E)-(28E) has a unique solution, which admits the representation

$$
\begin{gathered}
\boldsymbol{\beta}=\mathcal{M}\left(\nabla \psi_{0}^{*}, \boldsymbol{\lambda}^{*}\right)+\mathfrak{F}_{\beta}, \\
\overline{\boldsymbol{\lambda}}=\mathcal{L}\left(\nabla \psi_{0}^{*}, \boldsymbol{\lambda}^{*}\right)+\mathfrak{F}_{\lambda}, \\
p=\mathcal{P}\left(\nabla \psi_{0}^{*}, \boldsymbol{\lambda}^{*}\right)+\mathfrak{F}_{p} .
\end{gathered}
$$

Here the constant vectors $\mathfrak{F}_{\beta}, \mathfrak{F}_{\lambda}, \mathfrak{F}_{p}$ and the linear functionals $\mathcal{M}, \mathcal{L}, \mathcal{P}$ admit the estimates

$$
\begin{gathered}
\left|\mathfrak{F}_{\beta}\right| \leq c\left(|| \bar{F}_{2}|+| \bar{F}_{3}|+| f_{5} \mid\right) \\
\left|\mathfrak{F}_{\beta}\right|+\left|\mathfrak{F}_{\lambda}\right|+\left|\mathfrak{F}_{p}\right| \leq c\left(\left|F_{2}\right|_{-s}+\left|F_{3}\right|_{-s}+\left|f_{5}\right|\right) \\
|\mathcal{M}|+|\mathcal{L}|+|\mathcal{P}| \leq c\left(\varepsilon_{0}+r_{0}\right)\left(\left|\psi^{*}\right|_{-s}+\left|\lambda^{*}\right|_{-s}\right)
\end{gathered}
$$

where $s>0$ is an arbitrary number and $c$ is independent on $\varepsilon$ and $r$.

Proof. Let us consider the system of linear algebraic equations

$$
\begin{aligned}
\boldsymbol{\Omega} \overline{\boldsymbol{\lambda}}+\overline{\mathbf{T}} \delta \boldsymbol{\beta}+p \mathbf{e}_{1} & =\mathbf{a}, \\
\overline{\mathbf{T}}^{\top} \overline{\boldsymbol{\lambda}}+\overline{\mathbf{S}} \delta \boldsymbol{\beta} & =\mathbf{b}, \\
\overline{\lambda_{1}} & =f_{5} .
\end{aligned}
$$

Rewrite the first two equations in the form

$$
\begin{array}{r}
\boldsymbol{\Omega \boldsymbol { \lambda }}+\overline{\mathbf{T}_{0}} \delta \boldsymbol{\beta}+p \mathbf{e}_{1}=\mathbf{a}+\left(\mathbf{T}_{0}-\overline{\mathbf{T}}\right) \delta \boldsymbol{\beta}, \\
\mathbf{T}_{0}^{\top} \overline{\boldsymbol{\lambda}}+\mathbf{S}_{0} \delta \boldsymbol{\beta}=\mathbf{b}+\left(\mathbf{T}_{0}^{\top}-\overline{\mathbf{T}}^{\top}\right) \overline{\boldsymbol{\lambda}}+\left(\mathbf{S}_{0}-\overline{\mathbf{S}}\right) \delta \boldsymbol{\beta} .
\end{array}
$$

Notice that

$$
\overline{\boldsymbol{\lambda}}=\bar{\lambda}_{2} \mathbf{e}_{2}+f_{5} \mathbf{e}_{1}
$$

where $\mathbf{e}_{i}$ are the basis vectors with the components $e_{i j}=\delta_{i j}$. Thus we get the linear system of the equations for $\overline{\lambda_{2}}$ and $\delta \boldsymbol{\beta}$

$$
\begin{array}{r}
\overline{\lambda_{2}}+\mathbf{t}_{0} \cdot \delta \boldsymbol{\beta}=a_{2}+\left\{\left(\mathbf{T}_{0}-\overline{\mathbf{T}}\right) \delta \boldsymbol{\beta}\right\}_{2}, \\
\mathbf{S}_{0} \delta \boldsymbol{\beta}+\lambda_{2} \mathbf{t}_{0}=\mathbf{b}+f_{5}\left(\mathbf{T}_{0}^{\top}-\overline{\mathbf{T}}^{\top}\right) \mathbf{e}_{1}+ \\
\bar{\lambda}_{2}\left(\mathbf{T}_{0}^{\top}-\overline{\mathbf{T}}^{\top}\right) \mathbf{e}_{2}+\left(\mathbf{S}_{0}-\overline{\mathbf{S}}\right) \delta \boldsymbol{\beta} .
\end{array}
$$


Express $\overline{\lambda_{2}}$ in terms of $\delta \boldsymbol{\beta}$ using the first equation in (37E). Substituting the result into the second equation in (37E) we obtain the following equation for $\delta \beta$

$$
\begin{array}{r}
\mathbf{K}_{0} \delta \boldsymbol{\beta}=\left(\mathbf{S}_{0}-\overline{\mathbf{S}}\right) \delta \boldsymbol{\beta}+\left\{\left(\mathbf{T}_{0}-\overline{\mathbf{T}}\right) \delta \boldsymbol{\beta}\right\}_{2}\left(\left(\mathbf{T}_{0}^{\top}-\overline{\mathbf{T}}^{\top}\right) \boldsymbol{e}_{2}-\mathbf{t}_{0}\right)-\delta \boldsymbol{\beta} \cdot \mathbf{t}_{0}\left(\left(\mathbf{T}_{0}^{\top}-\overline{\mathbf{T}}^{\top}\right) \boldsymbol{e}_{2}\right. \\
+\left(\mathbf{b}-a_{2} \mathbf{t}_{0}+a_{2}\left(\mathbf{T}_{0}^{\top}-\overline{\mathbf{T}}^{\top}\right) \boldsymbol{e}_{2}+f_{5}\left(\mathbf{T}_{0}^{\top}-\overline{\mathbf{T}}^{\top}\right) \boldsymbol{e}_{1}\right)
\end{array}
$$

where the matrix $\mathbf{K}_{0}=\mathbf{S}_{0}-\mathbf{t}_{0} \otimes \mathbf{t}_{0}$ has a bounded inverse. Thus we get the following equation for $\delta \boldsymbol{\beta}$

$$
\delta \boldsymbol{\beta}-\mathbf{A} \delta \boldsymbol{\beta}=\mathbf{K}_{0}^{-1}\left(\mathbf{b}-a_{2} \mathbf{t}_{0}+a_{2}\left(\mathbf{T}_{0}^{\top}-\overline{\mathbf{T}}^{\top}\right) \boldsymbol{e}_{2}+f_{5}\left(\mathbf{T}_{0}^{\top}-\overline{\mathbf{T}}^{\top}\right) \boldsymbol{e}_{1}\right),
$$

where the linear mapping $\mathbf{A}: \mathbb{C}^{2} \rightarrow \mathbb{C}^{2}$ is given by

$$
\mathbf{A}: \delta \boldsymbol{\beta} \mapsto \mathbf{K}_{0}^{-1}\left\{\left(\mathbf{S}_{0}-\overline{\mathbf{S}}\right) \delta \boldsymbol{\beta}+\left\{\left(\mathbf{T}_{0}-\overline{\mathbf{T}}\right) \delta \boldsymbol{\beta}\right\}_{2}\left(\left(\mathbf{T}_{0}^{\top}-\overline{\mathbf{T}}^{\top}\right) \boldsymbol{e}_{2}-\mathbf{t}_{0}\right)-\delta \boldsymbol{\beta} \cdot \mathbf{t}_{0}\left(\left(\mathbf{T}_{0}^{\top}-\overline{\mathbf{T}}^{\top}\right) \boldsymbol{e}_{2}\right\}\right.
$$

In view of inequalities (3E), the mapping $\mathbf{A}$ admits the estimate $|\mathbf{A} \boldsymbol{\delta} \beta| \leq$ $c\left(\varepsilon_{0}+r_{0}\right)|\delta \boldsymbol{\beta}|$. Choosing $\varepsilon_{0}$ and $r_{0}$ so small that $c\left(\varepsilon_{0}+r_{0}\right) \leq 1 / 2$, we obtain that equation (39E) has the only solution which admits the representation

$$
\delta \boldsymbol{\beta}=(1-\mathbf{A})^{-1} \mathbf{K}_{0}^{-1}\left(\mathbf{b}-a_{2} \mathbf{t}_{0}+a_{2}\left(\mathbf{T}_{0}^{\top}-\overline{\mathbf{T}}^{\top}\right) \boldsymbol{e}_{2}+f_{5}\left(\mathbf{T}_{0}^{\top}-\overline{\mathbf{T}}^{\top}\right) \boldsymbol{e}_{1}\right)
$$

When system (39E) is solved the vector $\overline{\boldsymbol{\lambda}}$ and the scalar $p$ are restored by the relations

$$
\begin{gathered}
\overline{\boldsymbol{\lambda}}=f_{5} \mathbf{e}_{1}+\left\{a_{2}+\left(\left(\mathbf{T}_{0}-\overline{\mathbf{T}}\right) \boldsymbol{\beta}\right) \cdot \mathbf{e}_{2}\right\} \mathbf{e}_{2}-\mathbf{t}_{0} \cdot \delta \boldsymbol{\beta} \mathbf{e}_{2} \\
p=a_{1}+k f_{5}+\left\{\left(\mathbf{T}_{0}-\overline{\mathbf{T}}\right) \boldsymbol{\beta}\right\}_{1}
\end{gathered}
$$

In view of (27E) $-(28 \mathrm{E})$ the vectors $\boldsymbol{\beta}, \boldsymbol{\lambda}$ and the constant $p$ satisfy equations (36E) with the right hand sides

$$
\mathbf{a}=\overline{F_{2}}-\overline{\mathbf{T} \nabla \psi_{0}}, \quad \mathbf{b}=\overline{F_{3}}-\overline{\mathbf{S} \nabla \psi_{0}}-\overline{\mathbf{T}^{\top} \boldsymbol{\lambda}^{*}}
$$

Substituting these expressions into (39E)-(41E) we obtain desired representation (32Ea)-(32Ec) in which

$$
\begin{gathered}
\mathfrak{F}_{\beta}=(1-\mathbf{A})^{-1} \mathbf{K}_{0}^{-1}\left(\overline{F_{3}}-\left(\bar{F}_{2} \cdot \mathbf{e}_{2}\right) \overline{\mathbf{t}}_{0}+\left(\bar{F}_{2} \cdot \mathbf{e}_{2}\right)\left(\mathbf{T}_{0}^{\top}-\overline{\mathbf{T}}^{\top}\right) \mathbf{e}_{2}\right. \\
\left.+f_{5}\left(\mathbf{T}_{0}^{\top}-\overline{\mathbf{T}}^{\top}\right) \mathbf{e}_{1}\right) \\
\mathfrak{F}_{\lambda}=f_{5} \mathbf{e}_{1}+\bar{F}_{2} \mathbf{e}_{2}+\left(\left(\mathbf{T}_{0}-\overline{\mathbf{T}}\right) \mathfrak{F}_{\mu} \cdot \mathbf{e}_{2}\right) \mathbf{e}_{2}-\left(\mathbf{t}_{0} \cdot \mathfrak{F}_{\mu}\right) \mathbf{e}_{2}, \\
\mathfrak{F}_{p}=k f_{5}+\bar{F}_{2}+\left(\mathbf{T}_{0}-\overline{\mathbf{T}}\right) \mathfrak{F}_{\mu} \cdot \mathbf{e}_{1}
\end{gathered}
$$


and

$$
\begin{gathered}
\mathcal{M}=(1-\mathbf{A})^{-1} \mathbf{K}_{0}^{-1}\left\{\left(\overline{\mathbf{T} \nabla \psi_{0}^{*}} \cdot \mathbf{e}_{2}\right) \mathbf{t}_{0}-\left(\overline{\mathbf{T} \nabla \psi_{0}^{*}} \cdot \mathbf{e}_{2}\right)\left(\mathbf{T}_{0}^{\top}-\overline{\mathbf{T}}^{\top}\right) \mathbf{e}_{2}\right. \\
\left.-\overline{\mathbf{S} \nabla \psi_{0}^{*}}-\overline{\mathbf{T} \boldsymbol{\lambda}^{*}}\right\} \\
\mathcal{L}=-\left(\overline{\mathbf{T} \nabla \psi_{0}^{*}} \cdot \mathbf{e}_{2}\right) \mathbf{e}_{2}-\left(\left(\mathbf{T}_{0}-\overline{\mathbf{T}}\right) \mathcal{M} \cdot \mathbf{e}_{2}\right) \mathbf{e}_{2}-\left(\mathbf{t}_{0} \cdot \mathcal{M}\right) \mathbf{e}_{2}, \\
\mathcal{P}=-\left(\overline{\mathbf{T} \nabla \psi_{0}^{*}}\right) \cdot \mathbf{e}_{2}+\left(\mathbf{T}_{0}-\overline{\mathbf{T}}\right) \mathcal{M} \cdot \mathbf{e}_{1} .
\end{gathered}
$$

It remains to prove that these vectors and functionals satisfy inequalities (34E)-(35E). We begin with the observation that $\mathfrak{F}_{\beta}, \mathfrak{F}_{\lambda}$, and $\mathfrak{F}_{p}$ are linear integral functionals with analytic kernels of the linear space of functions $F_{2}$, $F_{3}$ and constants $f_{5}$. We have

$$
\left|\mathfrak{F}_{\beta}\right| \leq c\left(\left|\bar{F}_{2}\right|+\left|\bar{F}_{3}\right|+\left|f_{5}\right|\right)
$$

which immediately gives (33E). In order to prove (34E) we note that the Cauchy inequality $|\overline{u v}| \leq|u|_{s}|v|_{-s}$ implies

$$
\left|\bar{F}_{i}\right|=\left|\overline{1 \cdot F_{i}}\right| \leq|1|_{s}\left|F_{i}\right|_{-s} \leq c\left|F_{i}\right|_{-s}
$$

gives (34E). Next notice that the $\mathcal{M}, \mathcal{L}$, and $\mathcal{P}$ are linear integral functionals with analytical kernels. In particular, they are continuous in every Banach space $H_{s}$. Hence estimate (35E) is almost trivial. We prove it for the functional $\mathcal{M}$. The same proof works for $\mathcal{L}$ and $\mathcal{P}$. In order to estimate $\mathcal{M}$, notice that

$$
\begin{aligned}
\left|\overline{\mathbf{T} \nabla \psi_{0}^{*}}\right|=\left|\overline{\left(\mathbf{T}-\mathbf{T}_{0}\right) \nabla \psi^{*}}\right| \leq\left|\mathbf{T}-\mathbf{T}_{0}\right|_{s+1}\left|\nabla \psi^{*}\right|_{-s-1} \\
\quad \leq c\left\|\mathbf{T}-\mathbf{T}_{0}\right\|_{\sigma, 0}\left|\psi^{*}\right|_{-s} \leq c r_{0}\left|\psi^{*}\right|_{-s}
\end{aligned}
$$

Repeating these arguments we obtain

$$
\left|\overline{\mathbf{S} \nabla \psi_{0}^{*}}\right|+\left|\overline{\mathbf{T} \boldsymbol{\lambda}^{*}}\right| \leq c r_{0}\left(\left|\psi^{*}\right|_{-s}+\left|\boldsymbol{\lambda}^{*}\right|_{-s}\right) .
$$

Combining this result withy the obvious inequality

$$
|\mathcal{M}| \leq c\left(\left|\overline{\mathbf{T} \nabla \psi_{0}^{*}}\right|+\left|\overline{\mathbf{S} \nabla \psi_{0}^{*}}\right|+\left|\overline{\mathbf{T} \boldsymbol{\lambda}^{*}}\right|\right)
$$

we obtain estimate (35E) for $\mathcal{M}$. 
Let us turn to the proof of Proposition E.5. Our strategy is the following. First, we use representations (32Ea)-(32Ec) in order to reduce the system (26E) (31E) to the closed system of equations for the deviations $\psi_{0}^{*}, \boldsymbol{\lambda}^{*}$, and $\chi^{*}$. Then we reduce the obtained system to an abstract operator equation. We solve this equation in the space of bounded functions by using contraction mapping principle. Finally we prove that the obtained solution is analytic. Let us consider the basic system of equations (29E)-(31E). Assume that $\varepsilon_{0}$ and $r_{0}$ meet all requirements of Lemma E.6. It follows from representations (32Ea) $-(32 \mathrm{Ec})$ in this lemma that we can rewrite equations (29E)-(31E) in the equivalent form

$$
\begin{gathered}
\boldsymbol{\partial} \psi_{0}^{*}=G_{1}^{*} \\
\mathbf{J} \boldsymbol{\partial} \boldsymbol{\lambda}^{*}+\mathbf{\Omega} \boldsymbol{\lambda}^{*}+\left(\mathbf{T} \nabla \psi_{0}^{*}\right)^{*}=G_{2}^{*}-\mathbf{T}^{*} \mathcal{M}\left(\psi_{0}^{*}, \boldsymbol{\lambda}^{*}\right), \\
-\boldsymbol{\partial} \boldsymbol{\chi}^{*}+\left(\mathbf{S} \nabla \psi_{0}^{*}\right)^{*}+\left(\mathbf{T}^{\top} \boldsymbol{\lambda}^{*}\right)=G_{3}^{*}-\mathbf{S}^{*} \mathcal{M}\left(\psi_{0}^{*}, \boldsymbol{\lambda}^{*}\right)-\left(\mathbf{T}^{\top}\right)^{*} \mathcal{L}\left(\psi_{0}^{*}, \boldsymbol{\lambda}^{*}\right),
\end{gathered}
$$

where

$$
G_{1}^{*}=F_{1}^{*}, \quad G_{2}^{*}=F_{2}^{*}-\mathbf{T}^{*} \mathfrak{F}_{\beta}, \quad G_{3}^{*}=F_{3}^{*}-\mathbf{S}^{*} \mathfrak{F}_{\beta}-\left(\mathbf{T}^{\top}\right)^{*} \mathfrak{F}_{\lambda}
$$

It is worth noting that equations (49E) and relations (32Ea)-(32Ec) forms the system of equations which is equivalent to equations (23Ea)-(23Ed) It follows from estimate $(\underline{34 \mathrm{E}})$ that

$$
\left\|G_{1}^{*}\right\|_{\sigma_{1}, 0}+\left\|G_{2}^{*}\right\|_{\sigma_{1}, 0}+\left\|G_{3}^{*}\right\|_{\sigma_{1}, 0} \leq c\left(\left\|F_{1}^{*}\right\|_{\sigma_{1}, 0}+\left\|F_{2}^{*}\right\|_{\sigma_{1}, 0}+\left\|F_{3}^{*}\right\|_{\sigma_{1}, 0}+\left|f_{5}\right|\right) .
$$

Now introduce the linear operator

$$
\Xi_{1}:\left(F_{1}^{*}, F_{2}^{*}, F_{3}^{*}\right) \rightarrow\left(\psi_{0}^{*}, \boldsymbol{\lambda}^{*}, \chi^{*}\right)
$$

which assigns to every vector $\left(F_{1}^{*}, F_{2}^{*}, F_{3}^{*}\right)$ the solution of the following system of equations

$$
\begin{gathered}
\boldsymbol{\partial} \psi_{0}^{*}=F_{1}^{*}, \\
\mathbf{J} \boldsymbol{\partial} \boldsymbol{\lambda}^{*}+\boldsymbol{\Omega} \boldsymbol{\lambda}^{*}+\left(\mathbf{T} \nabla \psi_{0}^{*}\right)^{*}=F_{2}^{*}, \\
-\boldsymbol{\partial} \boldsymbol{\chi}^{*}+\left(\mathbf{S} \nabla \psi_{0}^{*}\right)^{*}+\left(\mathbf{T}^{\top} \boldsymbol{\lambda}^{*}\right)=F_{3}^{*} .
\end{gathered}
$$

Denote by $\mathcal{Z}_{\sigma}$ the subspace of the Banach space $\mathcal{A}_{\sigma_{0}} \times \mathcal{A}_{\sigma_{0}}^{2} \times \mathcal{A}_{\sigma, 0}^{n-1}$ which consists of all functions with with zero mean value. Let us show that for every $0 \leq \sigma_{0}<\sigma_{1} \leq \sigma$, the operator $\Xi_{1}: \mathcal{Z}_{\sigma_{1}} \rightarrow \mathcal{Z}_{\sigma_{0}}$ is continuous and

$$
\left\|\Xi_{1}\left(F_{1}^{*}, F_{2}^{*}, F_{3}^{*}\right)\right\|_{Z_{\sigma_{0}}} \leq c\left(\sigma_{1}-\sigma_{0}\right)^{-4 n-6}\left\|\Xi_{1}\left(F_{1}^{*}, F_{2}^{*}, F_{3}^{*}\right)\right\|_{Z_{\sigma_{1}}}
$$


Choose an arbitrary $\left(F_{1}^{*}, F_{2}^{*}, F_{3}^{*}\right) \in \mathcal{Z}_{\sigma_{1}}$. Since the system (51E)-(53E) is triangular, the existence of solution to this system obviously follows from Lemmas E.2 and E.3. Next, estimate (7E) in Lemma E.2 implies

$$
\left\|\psi_{0}^{*}\right\|_{2 / 3 \sigma_{0}+1 / 3 \sigma_{1}, 0} \leq c\left(\sigma_{1}-\sigma_{0}\right)^{-n-2}\left\|F_{1}^{*}\right\|_{\sigma_{1}, 0} .
$$

Using this inequality and applying Lemma E.3 to equation (52E) we arrive at the estimate

$$
\begin{array}{r}
\left\|\boldsymbol{\lambda}^{*}\right\|_{2 / 3 \sigma_{0}+1 / 3 \sigma_{1}, 0} \leq c\left(\sigma_{1}-\sigma_{0}\right)^{-2 n-3}\left(\left\|\psi_{0}^{*}\right\|_{1 / 3 \sigma_{0}+2 / 3 \sigma_{1}, 0}+\left\|F_{2}^{*}\right\|_{1 / 3 \sigma_{0}+2 / 3 \sigma_{1}, 0}\right) \\
\leq c\left(\sigma_{1}-\sigma_{0}\right)^{-3 n-5}\left(\left\|F_{1}^{*}\right\|_{\sigma_{1}, 0}+\left\|F_{2}^{*}\right\|_{\sigma_{1}, 0}\right) .
\end{array}
$$

Finally applying Lemma E.2 to equation (53E) we obtain

$$
\begin{gathered}
\left\|\chi^{*}\right\|_{\sigma_{0}, 0} \leq c\left(\sigma_{1}-\sigma_{0}\right)^{-n-2}\left(\left\|\psi_{0}^{*}\right\|_{2 / 3 \sigma_{0}+1 / 3 \sigma_{1}, 0}+\left\|\boldsymbol{\lambda}^{*}\right\|_{2 / 3 \sigma_{0}+1 / 3 \sigma_{1}, 0}+\left\|F_{3}^{*}\right\|_{2 / 3 \sigma_{0}+1 / 3 \sigma_{1}, 0}\right) \\
\leq c\left(\sigma_{1}-\sigma_{0}\right)^{-4 n-6}\left(\left\|F_{1}^{*}\right\|_{\sigma_{1}, 0}+\left\|F_{2}^{*}\right\|_{\sigma_{1}, 0}+\left\|F_{3}\right\|_{\sigma_{1}, 0}\right) . \quad(57 \mathrm{E})
\end{gathered}
$$

Combining estimates (55E)-(57E) we obtain (54E). Now introduce the second linear operator

$$
\Xi_{2}:\left(\psi_{0}^{*}, \boldsymbol{\lambda}^{*}, \boldsymbol{\chi}^{*}\right) \rightarrow\left(0, \mathbf{T}^{*} \mathcal{M}\left(\psi_{0}^{*}, \boldsymbol{\lambda}^{*}\right), \mathbf{S}^{*} \mathcal{M}\left(\psi_{0}^{*}, \boldsymbol{\lambda}^{*}\right)+\left(\mathbf{T}^{\top}\right)^{*} \mathcal{L}\left(\psi_{0}^{*}, \boldsymbol{\lambda}^{*}\right)\right)^{\top}
$$

Since the embedding $\mathcal{A}_{0,0} \rightarrow H_{-s}$ is bounded for $s \geq 0$, estimate (35E) yields the inequality

$$
\left\|\Xi_{2}\left(\psi_{0}^{*}, \boldsymbol{\lambda}^{*}, \boldsymbol{\chi}^{*}\right)\right\|_{\mathcal{Z}_{\sigma}} \leq c\left(\varepsilon_{0}+r_{0}\right) \|\left.\left(\psi_{0}^{*}, \boldsymbol{\lambda}^{*}, \boldsymbol{\chi}^{*}\right)\right|_{\mathcal{Z}_{0}} .
$$

Now we can rewrite system (49E) in the form of the operator equation

$$
\left(\psi_{0}^{*}, \lambda^{*}, \chi^{*}\right)=\Xi_{1} \Xi_{2}\left(\psi_{0}^{*}, \lambda^{*}, \chi^{*}\right)+\Xi_{1}\left(G_{1}^{*}, G_{2}^{*}, G_{3}^{*}\right) .
$$

Estimates (54E), (58E), and the inequality $1 / 4<\sigma$ imply

$$
\left\|\Xi_{1} \Xi_{2}\left(\psi_{0}^{*}, \boldsymbol{\lambda}^{*}, \boldsymbol{\chi}^{*}\right)\right\|_{\mathcal{Z}_{0}} \leq c\left(\varepsilon_{0}+r_{0}\right)\left\|\left(\psi_{0}^{*}, \boldsymbol{\lambda}^{*}, \boldsymbol{\chi}^{*}\right)\right\|_{\mathcal{Z}_{0}}
$$

It follows that the norm of the operator $\Xi_{1} \Xi_{2}: \mathcal{Z}_{0} \rightarrow \mathcal{Z}_{0}$ does not exceed $c\left(\varepsilon_{0}+r_{0}\right)$. Choosing $\varepsilon_{0}$ and $r_{0}$ sufficiently small and applying the contraction mapping principle we conclude that operator equation (59E) has a unique solution in the space $\mathcal{Z}_{0}$. This solution satisfies the inequality

$$
\left\|\left(\psi_{0}^{*}, \boldsymbol{\lambda}^{*}, \boldsymbol{\chi}^{*}\right)\right\|_{\mathcal{Z}_{0}} \leq c\left\|\Xi_{1}\left(G_{1}^{*}, G_{2}^{*}, G_{3}^{*}\right)\right\|_{\mathcal{Z}_{0}} .
$$


Let us prove that the obtained solution is analytic. Since $1 / 4 \leq \sigma_{1}$, estimates (54E) with $\sigma_{0}=0$ and estimate (50E) imply the inequality

$$
\begin{aligned}
\left\|\Xi_{1}\left(G_{1}^{*}, G_{2}^{*}, G_{3}^{*}\right)\right\|_{\mathcal{Z}_{0}} \leq & \left\|\left(G_{1}^{*}, G_{2}^{*}, G_{3}^{*}\right)\right\|_{\mathcal{Z}_{\sigma_{1}}} \\
& \leq c\left(\left\|F_{1}^{*}\right\|_{\sigma_{1}, 0}+\left\|F_{2}^{*}\right\|_{\sigma_{1}, 0}+\left\|F_{3}^{*}\right\|_{\sigma_{1}, 0}+\left|f_{5}\right|\right) .
\end{aligned}
$$

Hence the solution to equation (59E) admits the estimate

$$
\left\|\left(\psi_{0}^{*}, \boldsymbol{\lambda}^{*}, \boldsymbol{\chi}^{*}\right)\right\|_{\mathcal{Z}_{0}} \leq c\left(\left\|F_{1}^{*}\right\|_{\sigma_{1}, 0}+\left\|F_{2}^{*}\right\|_{\sigma_{1}, 0}+\left\|F_{3}^{*}\right\|_{\sigma_{1}, 0}+\left|f_{5}\right|\right),
$$

which along with (58E) leads to the inequality

$$
\left\|\Xi_{2}\left(\psi_{0}^{*}, \boldsymbol{\lambda}^{*}, \boldsymbol{\chi}^{*}\right)\right\|_{\mathcal{Z}_{\sigma}} \leq c\left(\left\|F_{1}^{*}\right\|_{\sigma_{1}, 0}+\left\|F_{2}^{*}\right\|_{\sigma_{1}, 0}+\left\|F_{3}^{*}\right\|_{\sigma_{1}, 0}+\left|f_{5}\right|\right) .
$$

On the other hand, inequality (54E) yields

$$
\begin{aligned}
& \left\|\Xi_{1}\left(\Xi_{2}\left(\psi_{0}^{*}, \boldsymbol{\lambda}^{*}, \boldsymbol{\chi}^{*}\right)+\left(G_{1}^{*}, G_{2}^{*}, G_{3}^{*}\right)\right)\right\|_{\mathcal{Z}_{\sigma_{0}}} \\
& \quad \leq c\left(\sigma_{1}-\sigma_{0}\right)^{-4 n-6}\left(\left\|\Xi_{2}\left(\psi_{0}^{*}, \boldsymbol{\lambda}^{*}, \chi^{*}\right)\right\|_{\mathcal{Z}_{\sigma_{1}}}+\left\|\left(G_{1}^{*}, G_{2}^{*}, G_{3}^{*}\right)\right\|_{\mathcal{Z}_{\sigma_{1}}}\right)
\end{aligned}
$$

Combining this result with (63E) and $(\underline{50 \mathrm{E}})$ we finally obtain

$$
\begin{aligned}
\left\|\Xi_{1}\left(\Xi_{2}\left(\psi_{0}^{*}, \lambda^{*}, \chi^{*}\right)+\left(G_{1}^{*}, G_{2}^{*}, G_{3}^{*}\right)\right)\right\|_{\mathcal{Z}_{\sigma_{0}}} & \\
& \leq c\left(\sigma_{1}-\sigma_{0}\right)^{-4 n-6}\left(\left\|F_{1}^{*}\right\|_{\sigma_{1}, 0}+\left\|F_{2}^{*}\right\|_{\sigma_{1}, 0}+\left\|F_{3}^{*}\right\|_{\sigma_{1}, 0}+\left|f_{5}\right|\right)
\end{aligned}
$$

Using this result and equation (59E) we finally obtain that the solution to system (49E) admits the estimate

$$
\begin{aligned}
\left\|\psi_{0}^{*}\right\|_{\sigma_{0}, 0}+ & \left\|\boldsymbol{\lambda}^{*}\right\|_{\sigma_{0}, 0}+\left\|\boldsymbol{\chi}^{*}\right\|_{\sigma_{0}, 0} \\
& \leq c\left(\sigma_{1}-\sigma_{0}\right)^{-4 n-6} c\left(\left|f_{5}\right|+\left\|F_{1}\right\|_{\sigma_{1}, 0}+\left\|F_{2}\right\|_{\sigma_{1}, 0}+\left\|F_{3}\right\|_{\sigma_{1}, 0}\right)
\end{aligned}
$$

Hence we proof that for all sufficiently small $\varepsilon_{0}$ and $r_{0}$ system (49E) has an analytic solution satisfying inequality (64E). Next notice that the mean value $\bar{\chi}=f_{3}$ and the constant $q=f_{1}$. Recall that the vector $\delta \boldsymbol{\beta}$, the mean value $\boldsymbol{\lambda}^{*}$, and the constant $p$ are connected with the deviations $\nabla \psi^{*}, \boldsymbol{\lambda}^{*}$ and $\chi^{*}$ by relations (32Ea)-(32Ec). It follows from this relations and estimates (34E)- (35E) that

$$
\begin{aligned}
|\overline{\boldsymbol{\chi}}|+|\delta \boldsymbol{\beta}|+|\overline{\boldsymbol{\lambda}}|+|p|+|q| \leq c\left(\left|f_{1}\right|+\left|f_{3}\right|\right. & \left.+\left|f_{5}\right|+\left\|F_{1}\right\|_{\sigma_{1}, 0}+\left\|F_{2}\right\|_{\sigma_{1}, 0}+\left\|F_{3}\right\|_{\sigma_{1}, 0}\right) \\
& +c\left(\left\|\psi_{0}^{*}\right\|_{\sigma_{0}, 0}+\left\|\boldsymbol{\lambda}^{*}\right\|_{\sigma_{0}, 0}+\left\|\boldsymbol{\chi}^{*}\right\|_{\sigma_{0}, 0}\right)
\end{aligned}
$$


Combining this result with (64E) we obtain

$$
\begin{aligned}
|\overline{\boldsymbol{\chi}}| & +|\delta \boldsymbol{\beta}|+|\overline{\boldsymbol{\lambda}}|+|p|+|q| \\
& \leq c\left(\sigma_{1}-\sigma_{0}\right)^{-4 n-6}\left(\left|f_{1}\right|+\left|f_{3}\right|+\left|f_{5}\right|+\left\|F_{1}\right\|_{\sigma_{1}, 0}+\left\|F_{2}\right\|_{\sigma_{1}, 0}+\left\|F_{3}\right\|_{\sigma_{1}, 0}\right) .
\end{aligned}
$$

This result, estimate (64E), and decomposition (25E) imply desired estimate (24E). This completes the proof of Proposition E.5.

Now we consider the extended truncated system, which includes the extra equation for the matrix-valued function $\boldsymbol{\Gamma}$. This extended system is formulated as follows

$$
\begin{gathered}
\boldsymbol{\partial} \psi_{0}+q=F_{1}, \\
\mathbf{J} \boldsymbol{\partial} \boldsymbol{\lambda}+\mathbf{T} \boldsymbol{\mu}+p \mathbf{e}_{1}=F_{2}, \quad \boldsymbol{\mu}=\delta \boldsymbol{\beta}+\nabla \psi_{0} \\
-\boldsymbol{\partial} \boldsymbol{\chi}+\mathbf{S} \boldsymbol{\mu}+\mathbf{T}^{\top} \boldsymbol{\lambda}=F_{3}, \\
\overline{\psi_{0}}=f_{1}, \quad \overline{\boldsymbol{\chi}}=f_{3} \quad \overline{\lambda_{1}}=f_{5}, \\
\boldsymbol{\partial}(\mathbf{J} \boldsymbol{\Gamma})+\boldsymbol{\Omega} \boldsymbol{\Gamma}+(\boldsymbol{\Omega} \boldsymbol{\Gamma})^{\top}+ \\
\mathbf{U}_{i j} \frac{\partial \lambda_{i}}{\partial \xi_{j}}+\mu_{i} \mathbf{E}_{i}+\lambda_{i} \mathbf{K}_{i}+\delta \mathbf{M}=F_{4}, \\
\Gamma_{11}=-\Gamma_{22}, \quad \bar{\Gamma}_{12}=f_{4}, \quad \delta \mathbf{M}=\delta M \operatorname{diag}(1,0) .
\end{gathered}
$$

The following proposition guarantees the well-posedness of the truncated problem (65E).

Proposition E.7. Under the assumptions of Theorem E.1, there are $\varepsilon_{0}>0$ and $r_{0}$ with the following properties. For every

$$
\begin{gathered}
(\alpha, k) \in \Sigma_{\varrho}, \quad\|\boldsymbol{\varphi}-\boldsymbol{\varphi}(\alpha)\|_{\sigma, d} \leq r_{0}, \quad \boldsymbol{\varphi}(\alpha)=(0,0,0, \boldsymbol{\alpha}, 1,0,0), \quad|\varepsilon| \leq \varepsilon_{0}, \quad, \\
0 \leq \sigma_{0}<\sigma_{1}<\sigma, \quad \sigma_{1}>1 / 4 \\
\mathbf{f} \in \mathbb{C}^{4}, \quad \mathbf{F} \in \mathcal{F}_{\sigma_{1}, 0} .
\end{gathered}
$$

Then problem (65E) has a unique solution

$$
\left(\psi_{0}, \boldsymbol{\lambda}, \boldsymbol{\chi}, \boldsymbol{\Gamma}, \delta \boldsymbol{\beta}, q, p, \delta \mathbf{M}\right) \in \mathcal{A}_{\sigma_{0}, 0} \times \mathcal{A}_{\sigma_{0}, 0}^{2} \times \mathcal{A}_{\sigma, d}^{n-1} \times \mathcal{A}_{\sigma_{0}, 0}^{4} \times \mathbb{C}^{n-1} \times \mathbb{C}^{3}
$$


This solution admits the estimate

$$
\left\|\left(\psi_{0}, \boldsymbol{\lambda}, \boldsymbol{\chi}, \boldsymbol{\Gamma}\right)\right\|_{\sigma_{0}, 0}+|(\delta \boldsymbol{\beta}, \delta q, \delta p, \delta \mathbf{M})| \leq c\left(\sigma_{1}-\sigma_{0}\right)^{-8 n-12}\left(\|\mathbf{F}\|_{\sigma_{1}, 0}+|\mathbf{f}|\right)
$$

where the constant $c$ is independent of $\varepsilon_{0}, r_{0}$, and $\sigma$.

Proof. We begin with the observation that equations (65Ea)-(65Ed) are independent of $\Gamma$. Moreover, system (65Ea) - (65Ed) coincides with system (23Ea)(23Ed). Applying Proposition E.5 we conclude that this system has a unique analytic solution. This solution admits the estimate

$$
\begin{aligned}
\left\|\psi_{0}\right\|_{\sigma_{0}, 0}+\|\boldsymbol{\lambda}\|_{\sigma_{0}, 0}+\|\boldsymbol{\chi}\|_{\sigma_{0}, 0}+|p| & +|q| \\
& +|\delta \boldsymbol{\beta}| \leq c\left(\sigma_{1}-\sigma_{0}\right)^{-6 n-8}\left(\|\mathbf{F}\|_{\sigma_{1}, 0}+|\mathbf{f}|\right) .
\end{aligned}
$$

Hence it suffices to analyze equation (65Ee). Rewrite it in the form

$$
\boldsymbol{\partial}(\mathbf{J} \boldsymbol{\Gamma})+\boldsymbol{\Omega} \boldsymbol{\Gamma}+(\boldsymbol{\Omega} \boldsymbol{\Gamma})^{\top}+\delta \mathbf{M}=F_{4}-\mathbf{U}_{i j} \frac{\partial \lambda_{i}}{\partial \xi_{j}}-\mu_{i} \mathbf{E}_{i}-\lambda_{i} \mathbf{K}_{i}
$$

Applying inequality (67E) we obtain

$$
\begin{aligned}
& \left\|\mathbf{U}_{i j} \frac{\partial \lambda_{i}}{\partial \xi_{j}}-\mu_{i} \mathbf{E}_{i}-\lambda_{i} \mathbf{K}_{i}\right\|_{2 / 3 \sigma_{0}+1 / 3 \sigma_{1}, 0} \leq c\left(\sigma_{1}-\sigma_{0}\right)^{-1}\left(\|\boldsymbol{\lambda}\|_{1 / 2 \sigma_{0}+1 / 2 \sigma_{1}, 0}\right. \\
& \left.+c\left\|\psi_{0}\right\|_{1 / 2 \sigma_{0}+1 / 2 \sigma_{1}, 0}+|\delta \boldsymbol{\beta}|\right) \leq c\left(\sigma_{1}-\sigma_{0}\right)^{-6 n-9}\left(\|\mathbf{F}\|_{\sigma_{1}, 0}+|\mathbf{f}|\right) .
\end{aligned}
$$

Applying Lemma 17E we conclude that equation (68E) has a unique analytic solution which satisfies condition (65Ef) and admits the estimate

$$
\|\boldsymbol{\Gamma}\|_{\sigma_{0}, 0}+|\delta \mathbf{M}| \leq c\left(\sigma_{1}-\sigma_{0}\right)^{-2 n-3}\left(\left\|\mathbf{F}_{4}-\mathbf{U}_{i j} \frac{\partial \lambda_{i}}{\partial \xi_{j}}-\mu_{i} \mathbf{E}_{i}-\lambda_{i} \mathbf{K}_{i}\right\|_{2 / 3 \sigma_{0}+1 / 3 \sigma_{1}, 0}+\left|f_{4}\right|\right)
$$

It follows from this and (69E) that

$$
\|\boldsymbol{\Gamma}\|_{\sigma_{0}, 0}+|\delta \mathbf{M}| \leq c\left(\sigma_{1}-\sigma_{0}\right)^{-8 n-12}\left(\|\mathbf{F}\|_{\sigma_{1}, 0}+|\mathbf{f}|\right) .
$$

Combining this result with (67E) we obtain (66E) and the proposition follows. 
Proof of Theorem E.1 We are now in a position to complete the proof of TheoremE.1. The proof is based on the Proposition E.7 and the contraction mapping principle. By technical reasons it is convenient to introduce the following denotations. Introduce the vectors

$$
\mathfrak{w}=\left(\psi_{0}, \boldsymbol{\lambda}, \boldsymbol{\chi}, \boldsymbol{\Gamma}, \delta \boldsymbol{\beta}, q, p, \delta M\right), \quad \mathfrak{f}=\left(F_{1}, F_{2}, F_{3}, F_{4}, f_{1}, f_{3}, f_{4}, f_{5}\right) .
$$

Denote by $\mathcal{X}_{\sigma}$ the closed subspace of the Banach space

$$
\mathcal{A}_{\sigma_{0}, 0} \times \mathcal{A}_{\sigma_{0}, 0}^{2} \times \mathcal{A}_{\sigma, d}^{n-1} \times \mathcal{A}_{\sigma_{0}, 0}^{4} \times \mathbb{C}^{n-1} \times \mathbb{C}^{3},
$$

which consists of all vector functions $\mathfrak{w}$ such that $\operatorname{tr} \boldsymbol{\Gamma}=0$. Denote by $\mathcal{Y}_{\sigma}$ the closed subspace of the Banach space

$$
\mathcal{A}_{\sigma_{0}, 0} \times \mathcal{A}_{\sigma_{0}, 0}^{2} \times \mathcal{A}_{\sigma, d}^{n-1} \times \mathcal{A}_{\sigma_{0}, 0}^{4} \times \mathbb{C} \times \mathbb{C}^{n-1} \times \mathbb{C}^{n-1} \times \mathbb{C}
$$

which consists of all vectors $\mathfrak{f}$ such that the matrix $F_{4}$ is symmetric. Rewrite main system (1E) in the form.

$$
\begin{gathered}
\boldsymbol{\partial} \psi_{0}+q=F_{1}+\Xi_{3,1}(p, \delta M), \\
\mathbf{J} \boldsymbol{\partial}+\boldsymbol{\Omega} \boldsymbol{\lambda}+\mathbf{T} \boldsymbol{\mu}+p \mathbf{e}_{1}=F_{2}+\Xi_{3,2}(p, \delta M), \quad \boldsymbol{\mu}=\nabla \psi_{0}+\delta \boldsymbol{\beta} \\
-\boldsymbol{\partial} \boldsymbol{\chi}+\mathbf{S} \boldsymbol{\mu}+\mathbf{T}^{\top} \boldsymbol{\lambda}=F_{3}, \\
\boldsymbol{\partial}(\mathbf{J} \boldsymbol{\Gamma})+\boldsymbol{\Omega} \boldsymbol{\Gamma}+(\boldsymbol{\Omega} \boldsymbol{\Gamma})^{\top}+\mathbf{U}_{i j} \frac{\partial \lambda_{i}}{\partial \xi_{j}}+\mu_{i} \mathbf{E}_{i}+\lambda_{i} \mathbf{K}_{i}+\delta \mathbf{M}= \\
F_{4}+\Xi_{3,4}(\delta M), \quad \Gamma_{11}=-\Gamma_{22}, \\
\overline{\psi_{0}}=f_{1}+\Xi_{3,5}(\boldsymbol{\beta}, \boldsymbol{\lambda}) \\
\overline{\boldsymbol{\chi}}=f_{3}+\Xi_{3,6}(\boldsymbol{\chi}), \\
\overline{\Gamma_{12}}=f_{4}+\Xi_{3,7}(\boldsymbol{\Gamma}) \\
\overline{\lambda_{1}}=f_{5}+\Xi_{3,8}(\boldsymbol{\lambda}, \boldsymbol{\chi}),
\end{gathered}
$$

where

$$
\begin{gathered}
\Xi_{3,1}(p, \delta M)=-p\left(w_{1}-\alpha\right)-\frac{1}{2} \delta M\left(w_{1}-\alpha\right)^{2}, \\
\Xi_{3,2}(p, \delta M)=-p\left(\mathbf{W}^{\top}-\mathbf{I}\right) \mathbf{e}_{1}-\delta M\left(w_{1}-\alpha\right) \mathbf{W}^{\top} \mathbf{e}_{1}, \\
\Xi_{3,3}=0, \quad \Xi_{3,4}(\delta M)=\delta \mathbf{M}-\mathbf{W}^{\top} \delta \mathbf{M W}, \\
\Xi_{3,5}(\boldsymbol{\beta}, \boldsymbol{\lambda})=\boldsymbol{\delta} \beta \cdot \overline{\mathbf{u}}-\overline{w_{2} \mathbf{W} \boldsymbol{\lambda} \cdot \mathbf{e}_{1}} \\
\Xi_{3,6}(\boldsymbol{\chi})=\overline{\left(\mathbf{I}-\mathbf{V}^{-\top}\right) \boldsymbol{\chi}}, \quad \Xi_{3,7}(\boldsymbol{\Gamma})=\overline{((\mathbf{I}-\mathbf{W}) \boldsymbol{\Gamma})_{12}} \\
\Xi_{3,8}(\boldsymbol{\lambda}, \boldsymbol{\chi})=\overline{(\mathbf{I}-\mathbf{W}) \boldsymbol{\lambda} \cdot \mathbf{e}_{1}}-\overline{\boldsymbol{\chi} \cdot \nabla w_{1}} .
\end{gathered}
$$


It is necessary to prove that, under the assumptions of Theorem E.1, the system of equations (70E) for all $\mathfrak{f} \in \mathcal{Y}_{\sigma_{1}}$ has a unique solution $\mathfrak{w} \in \mathcal{X}_{\sigma_{0}}$ satisfying the inequality

$$
\|\mathfrak{w}\|_{\mathcal{X}_{\sigma_{0}}} \leq c\left(\sigma_{1}-\sigma_{0}\right)^{-8 n-12}\|\mathfrak{f}\|_{\mathcal{Y}_{\sigma_{1}}}
$$

Recall that

$$
0 \leq \sigma_{0}<\sigma_{1} \leq \sigma, \quad 1 / 4 \leq \sigma_{1} .
$$

The proof is based on the following auxiliary lemma.

Lemma E.8. Under the assumptions of Theorem E.1 the operator $\Xi_{3}=$ $\left(\Xi_{3, i}\right)_{1 \leq i \leq 8}$ admits the estimate

$$
\left\|\Xi_{3}(\mathfrak{w})\right\|_{\mathcal{Y}_{\sigma}} \leq c r_{0}\|\mathfrak{w}\|_{\mathcal{X}_{0}}
$$

Proof. Obviously we have

$$
\begin{array}{r}
\left\|\Xi_{3,1}(p, \delta M)\right\|_{\mathcal{Y}_{\sigma}}+\left\|\Xi_{3,2}(p, \delta M)\right\|_{\mathcal{Y}_{\sigma}}+\left\|\Xi_{3,4}(\delta M)\right\|_{\mathcal{Y}_{\sigma}} \leq \\
c(|p|+|\delta M|)\left(\left\|w_{1}-\alpha\right\|_{\sigma, 0}+\|\mathbf{W}-\mathbf{I}\|_{\sigma, 0}\right)
\end{array}
$$

It follows from the estimate

$$
(\alpha, k) \in \Sigma_{\varrho}, \quad\|\boldsymbol{\varphi}-\boldsymbol{\varphi}(\alpha)\|_{\sigma, d} \leq r, \quad \boldsymbol{\varphi}(\alpha)=(0,0,0, \boldsymbol{\alpha}, 1,0,0),
$$

in condition of Theorem E.1 and the relations $\boldsymbol{\varphi}=\left(\boldsymbol{\beta}, \varphi_{0}, \mathbf{u}, \mathbf{w}, W_{11}, W_{12}, W_{21}\right)$, $\operatorname{det} \mathbf{W}=1$ that

$$
\|\mathbf{u}\|_{\sigma, 0}+\left\|w_{2}\right\|_{\sigma, 0}+\left\|w_{1}-\alpha\right\|_{\sigma, 0}+\|\mathbf{W}-\mathbf{I}\|_{\sigma, 0} \leq c r \leq c r_{0}
$$

Thus we get

$$
\left\|\Xi_{3,1}(p, \delta M)\right\|_{\mathcal{Y}_{\sigma}}+\left\|\Xi_{3,2}(p, \delta M)\right\|_{\mathcal{Y}_{\sigma}}+\left\|\Xi_{3,4}(\delta M)\right\|_{\mathcal{Y}_{\sigma}} \leq c r_{0}(|p|+|\delta M|)
$$

Next, the Cauchy inequality implies

$$
\begin{aligned}
\left|\Xi_{3,5}\right|+\left|\Xi_{3,6}\right|+\left|\Xi_{3,7}\right|+\left|\Xi_{3,8}\right| \leq c\left(|\delta \boldsymbol{\beta}|+|\boldsymbol{\lambda}|_{0}+|\boldsymbol{\chi}|_{0}+|\boldsymbol{\Gamma}|_{0}\right) \times \\
\left(|\mathbf{u}|_{0}+|\mathbf{W}-\mathbf{I}|_{0}+\left|w_{2} \mathbf{W}\right|_{0}+\left|\nabla w_{1}\right|_{0}+\left|\mathbf{I}-\mathbf{V}^{-1}\right|_{0}\right)
\end{aligned}
$$

Recall that $I-\mathbf{V}^{-\top} \equiv-\mathbf{u}^{\prime}$ and the embedding

$$
\mathcal{A}_{\sigma, 0} \hookrightarrow H_{1} \hookrightarrow H_{0}, \quad \mathcal{A}_{0,0} \hookrightarrow H_{0}
$$


is bounded. From this and (74E) we obtain

$$
\begin{aligned}
\left|\mathbf{u}^{\prime}\right|_{0}+|\mathbf{W}-\mathbf{I}|_{0}+ & \left|w_{2} \mathbf{W}\right|_{0}+\left|\nabla w_{1}\right|_{0}+\left|\mathbf{I}-\mathbf{V}^{-\top}\right|_{0} \\
\leq & |\mathbf{u}|_{1}+|\mathbf{W}-\mathbf{I}|_{0}+\left|w_{2} \mathbf{W}\right|_{0}+\left|w_{1}^{*}\right|_{1} \\
& \leq\|\mathbf{u}\|_{\sigma, 0}+\|\mathbf{W}-\mathbf{I}\|_{\sigma, 0}+\left\|w_{2} \mathbf{W}\right\|_{\sigma, 0}+\left\|w_{1}^{*}\right\|_{\sigma, 0} \leq c r_{0}
\end{aligned}
$$

and

$$
|\boldsymbol{\lambda}|_{0}+|\boldsymbol{\chi}|_{0}+|\boldsymbol{\Gamma}|_{0} \leq c\left(\|\boldsymbol{\lambda}\|_{0,0}+\|\boldsymbol{\chi}\|_{0,0}+\|\boldsymbol{\Gamma}\|_{0,0}\right) .
$$

Combining the obtained results we arrive at the estimate

$$
\left|\Xi_{3,5}\right|+\left|\Xi_{3,6}\right|+\left|\Xi_{3,7}\right|+\left|\Xi_{3,8}\right| \leq c r_{0}\left(|\boldsymbol{\beta}|+\|\boldsymbol{\lambda}\|_{0,0}+\|\boldsymbol{\chi}\|_{0,0}+\|\boldsymbol{\Gamma}\|_{0,0}\right) .
$$

It remains to note that the desired estimate (73E) obviously follows from (75E) and (76E).

Let us turn to the proof of the Theorem E.1. Denote by $\Xi_{4}$ the linear operator which assigns to every $\mathfrak{f} \in Y_{\sigma_{1}}$ the solution $\mathfrak{w}$ of problem (65E). It follows from the Proposition (E.7) that for small $\varepsilon_{0}$ and $r_{0}$, the operator $\Xi_{4}: \mathcal{Y}_{\sigma_{1}} \rightarrow \mathcal{X}_{\sigma_{0}}$ is bounded and

$$
\left\|\Xi_{4} \mathfrak{f}\right\|_{\sigma_{0}} \leq c\left(\sigma_{1}-\sigma_{0}\right)^{-6 n-8}\|\mathfrak{f}\|_{\mathcal{Y}_{\sigma_{1}}} .
$$

In particular, we have

$$
\left\|\Xi_{4} \mathfrak{f}\right\|_{\sigma_{0}} \leq c\|\mathfrak{f}\|_{\mathcal{Y}_{\sigma_{1}}}
$$

Now we can rewrite system (70E) in the form of the operator equation

$$
\mathfrak{w}=\Xi_{4}\left(\Xi_{3} \mathfrak{w}+\mathfrak{f}\right) .
$$

By virtue of $(\overline{73 \mathrm{E}})$ and $(\overline{78 \mathrm{E}})$ the operator $\Xi_{4} \Xi_{3}: \mathcal{X}_{0} \rightarrow \mathcal{X}_{0}$ is bounded and its norm does not exceed $c r_{0}$. Choosing $r_{0}$ sufficiently small and applying the contraction mapping principle we conclude that operator equation (79E) has a unique solution in the space $\mathcal{X}_{0}$. In view of $(78 \mathrm{E})$, this solution admits the estimate

$$
\|\mathfrak{w}\|_{\mathcal{X}_{0}} \leq c\|\mathfrak{f}\|_{Y_{\sigma_{1}}}
$$

Let us prove that this solution is analytic. Notice that estimate (73E) implies

$$
\left\|\Xi_{3} \mathfrak{w}\right\|_{\mathcal{Y}_{\sigma_{1}}} \leq c\|\mathfrak{w}\|_{\mathcal{X}_{0}} \leq c\|\mathfrak{f}\|_{Y_{\sigma_{1}}}
$$

Hence

$$
\left\|\Xi_{3} \mathfrak{w}+\mathfrak{f}\right\|_{\mathcal{Y}_{\sigma_{1}}} \leq c\|\mathfrak{w}\|_{\mathcal{X}_{0}}+\|\mathfrak{f}\|_{\mathcal{Y}_{\sigma_{1}}} \leq c\|\mathfrak{f}\|_{Y_{\sigma_{1}}}
$$

Combining this result with (77E) we obtain desired estimate (71E). This completes the proof of Theorem E.1. 


\section{E.2 Proof of Theorem 8.1}

We split the proof into two parts. First we prove the existence and uniqueness of solutions to problem (8.5),

$$
\begin{gathered}
\boldsymbol{\partial} \boldsymbol{\mu}=-p \nabla w_{1}-\nabla g_{1}, \\
\mathbf{J} \boldsymbol{\partial}+\boldsymbol{\Omega} \boldsymbol{\lambda}+\mathbf{T} \boldsymbol{\mu}+p \mathbf{W}^{\top} \mathbf{e}_{1}=\mathbf{g}_{2} \\
-\boldsymbol{\partial} \boldsymbol{\lambda}+\mathbf{S} \boldsymbol{\mu}+\mathbf{T}^{\top} \boldsymbol{\lambda}=0 \\
\overline{\{W \boldsymbol{\lambda}\} \cdot \mathbf{e}_{1}}+\overline{\left\{\boldsymbol{\chi} \cdot \nabla w_{1}\right\}}=\gamma, \\
\overline{\left\{\mathbf{V}^{-\top} \boldsymbol{\chi}\right\}}=0 .
\end{gathered}
$$

in the space of analytic function and establish estimate (8.9). Next, we estimate the obtained solution in the Sobolev space $H_{s}$. We begin with the observation that problem $(80 \mathrm{E})$ is the very particular case of general problem (1E). Notice that, in view of conditions (8.6) and (8.7) of Theorem 8.1, the matrices $\mathbf{W}, \mathbf{V}, \mathbf{S}, \mathbf{T}$ and the function $w_{1}$ meet all requirements of Theorem E.1 with $\sigma$ replaced by $\sigma / 2$ and $r_{0}=c \varepsilon_{0}$. Next set

$$
\begin{aligned}
& \mathbf{U}_{i j}=0, \quad \mathbf{E}_{i}=0, \quad \mathbf{K}_{i}=0, \\
& \sigma_{0}=\sigma / 4, \quad \sigma_{1}=\sigma / 2 \geq 1 / 4
\end{aligned}
$$

Obviously these quantities satisfy all conditions of Theorem E.1. Now introduce the temporary notation

$$
\begin{array}{r}
F_{1}=-g_{1}^{*}, \quad F_{2}=\mathbf{g}_{2}, \quad F 3=0, \quad F_{4}=0, \\
f_{1}=0, \quad f_{3}=0, \quad f_{4}=0, \quad f_{5}=\gamma,
\end{array}
$$

Since $r_{0} \leq c \varepsilon_{0}$, it follows from Theorem E.1 that for a suitable choice of $\varepsilon_{0}$ and for all $|\varepsilon| \leq \varepsilon_{0}$, the equations (1E) with the righthand sides (82E) have a unique analytic solution. This solution satisfies inequalities (5E). In particular, we have

$$
\left|\left(\psi_{0}, \boldsymbol{\lambda}, \boldsymbol{\chi}\right) \|_{3 / 8 \sigma, 0}+\right|(\delta \boldsymbol{\beta}, p) \mid \leq c\left(\left\|g_{1}^{*}\right\|_{\sigma / 2,0}+\left\|\mathbf{g}_{2}^{*}\right\|_{\sigma / 2,0}+|\gamma|\right) .
$$

By virtue of condition (81E) and the equalities $f_{4}=0, F_{4}=0$, the matrices $\boldsymbol{\Gamma}$ and $\delta \mathbf{M}$ satisfy the homogeneous equation

$$
\boldsymbol{\partial}(\mathbf{J} \Gamma)+\Omega \Gamma+(\Omega \Gamma)^{\top}+\delta \mathbf{M}=\delta \mathbf{M}-\mathbf{W}^{\top} \delta \mathbf{M W}
$$




$$
\overline{\Gamma_{12}}=\overline{((\mathbf{I}-\mathbf{W}) \mathbf{\Gamma})_{12}}
$$

Applying Lemma E.3 and the contraction mapping principle we conclude that $\boldsymbol{\Gamma}=\delta \mathbf{M}=0$. Since $\bar{F}_{1}=0$, we also have $q=0$. Hence the functions $\psi_{0}, \boldsymbol{\lambda}$ and $\boldsymbol{\chi}$ satisfy the equations

$$
\begin{gathered}
\boldsymbol{\partial} \psi_{0}=-p w_{1}^{*}-g_{1}^{*}, \\
\mathbf{J} \boldsymbol{\partial}+\boldsymbol{\Omega} \boldsymbol{\lambda}+\mathbf{T} \boldsymbol{\mu}+p \mathbf{W}^{\top} \mathbf{e}_{1}=\mathbf{g}_{2} \\
\boldsymbol{\mu}=\delta \boldsymbol{\beta}+\nabla \psi_{0}, \\
-\boldsymbol{\partial} \boldsymbol{\lambda}+\mathbf{S} \boldsymbol{\mu}+\mathbf{T}^{\top} \boldsymbol{\lambda}=0 \\
\overline{\{W \boldsymbol{\lambda}\} \cdot \mathbf{e}_{1}}+\overline{\left\{\boldsymbol{\chi} \cdot \nabla w_{1}\right\}}=\gamma, \\
\overline{\left\{\mathbf{V}^{-\top} \boldsymbol{\chi}\right\}}=0 .
\end{gathered}
$$

It follows that $\boldsymbol{\mu}, \boldsymbol{\lambda}, \boldsymbol{\chi}$ and $p$ satisfy equations (80E). Inequalities (83E) and the obvious inequality

$$
\|\boldsymbol{\mu}\|_{\sigma / 4,0} \leq c\left(|\boldsymbol{\beta}|+\left\|\psi_{0}\right\|_{3 / 8 \sigma, 0}\right.
$$

implies the estimate

$$
\left|(\boldsymbol{\mu}, \boldsymbol{\lambda}, \boldsymbol{\chi}) \|_{\sigma / 4,0}+\right| p \mid \leq c\left(\left\|g_{1}^{*}\right\|_{\sigma / 2,0}+\left\|\mathbf{g}_{2}^{*}\right\|_{\sigma / 2,0}+|\gamma|\right) .
$$

which yields (8.9). It remains to estimate the solution to problem (80E) in the Sobolev spaces. Now we change the denotations and set

$$
\begin{gathered}
F_{1}=-p w_{1}^{*}-g_{1} *, \quad F_{2}=p\left(\mathbf{I}-\mathbf{W}^{\top}\right) \mathbf{e}_{1}+\boldsymbol{g}_{2}, \quad F_{3}=0, \\
f_{1}=0, \quad f_{3}=\overline{\left(\mathbf{I}-\mathbf{V}^{-\top}\right) \boldsymbol{\chi}} \quad f_{5}=\overline{(\mathbf{I}-\mathbf{W}) \boldsymbol{\lambda} \mathbf{e}_{1}}-\overline{\boldsymbol{\chi} \cdot \nabla w_{1}}
\end{gathered}
$$

Now we estimate these quantities in the Sobolev space $H_{s}$. Obviously we have

$$
\begin{array}{r}
\left|F_{1}\right|_{s} \leq|p|\left|w_{1}^{*}\right|_{s}+\left|g_{1}^{*}\right|_{s} \leq c \varepsilon_{0}|p|+\left|g_{1}^{*}\right|_{s} \\
\left|F_{2}\right|_{s} \leq|p||\mathbf{I}-\mathbf{W}|_{s}+\left|\mathbf{g}_{2}\right|_{s} \leq c \varepsilon_{0}|p|+\left|\mathbf{g}_{2}\right|_{s}
\end{array}
$$

We also have

$$
\left|\bar{F}_{2}\right| \leq c \varepsilon_{0}|p|+\left|\overline{\mathbf{g}_{2}}\right|
$$


The Cauchy inequality implies

$$
\begin{array}{r}
\left|f_{3}\right| \leq\left|\mathbf{I}-\mathbf{V}^{-\top}\right|_{-s}|\boldsymbol{\chi}|_{s} \leq\left\|\mathbf{I}-\mathbf{V}^{-\top}\right\|_{\sigma / 2,0}|\boldsymbol{\chi}|_{s} \leq c \varepsilon_{0}|\boldsymbol{\chi}|_{s} \\
\left|f_{5}\right| \leq|\mathbf{I}-\mathbf{W}|_{-s}|\boldsymbol{\lambda}|_{s}+\left|\nabla w_{1}\right|_{-s}|\boldsymbol{\chi}|_{s} \leq \\
c\|\mathbf{I}-\mathbf{W}\|_{\sigma / 2,0}|\boldsymbol{\lambda}|_{s}+\left\|w_{1}^{*}\right\|_{\sigma / 2,0}|\boldsymbol{\chi}|_{s} \leq c \varepsilon_{0}\left(|\boldsymbol{\lambda}|_{s}+|\boldsymbol{\chi}|_{s}\right)
\end{array}
$$

Combining the obtained results we arrive at the inequality

$$
\left|f_{3}\right|+\left|f_{5}\right| \leq c \varepsilon_{0}(|\overline{\boldsymbol{\lambda}}|+|\overline{\boldsymbol{\chi}}|)+c \varepsilon_{0}\left(\left|\boldsymbol{\lambda}^{*}\right|_{s}+\left|\chi^{*}\right|_{s}\right)
$$

With this notation equations $(84 \mathrm{E})$ can be written in the form

$$
\begin{gathered}
\boldsymbol{\partial} \psi_{0}+q=F_{1}, \\
\mathbf{J} \boldsymbol{\partial} \boldsymbol{\lambda}+\boldsymbol{\Omega} \boldsymbol{\lambda}+\mathbf{T} \boldsymbol{\mu}+p \mathbf{e}_{1}=F_{2}, \quad \boldsymbol{\mu}=\delta \boldsymbol{\beta}+\nabla \psi_{0} \\
-\boldsymbol{\partial} \boldsymbol{\chi}+\mathbf{S} \boldsymbol{\mu}+\mathbf{T}^{\top} \boldsymbol{\lambda}=F_{3}, \\
\overline{\psi_{0}}=f_{1}, \quad \overline{\boldsymbol{\chi}}=f_{3}, \quad \overline{\lambda_{1}}=f_{5} .
\end{gathered}
$$

Hence the these equations coincide with equations (23E) at least formally. It follows from Lemma E.6 that a solution to system (80E) admits the representation (32E). recalling identities $\boldsymbol{\beta}=\overline{\boldsymbol{\mu}}$ and $\nabla \psi_{0}=\boldsymbol{\mu}^{*}$ we can rewrite this representation in the form

$$
\begin{gathered}
\overline{\boldsymbol{\mu}}=\mathcal{M}\left(\boldsymbol{\mu}^{*}, \boldsymbol{\lambda}^{*}\right)+\mathfrak{F}_{\beta}, \\
\overline{\boldsymbol{\lambda}}=\mathcal{L}\left(\boldsymbol{\mu}^{*}, \boldsymbol{\lambda}^{*}\right)+\mathfrak{F}_{\lambda}, \\
p=\mathcal{P}\left(\boldsymbol{\mu}^{*}, \boldsymbol{\lambda}^{*}\right)+\mathfrak{F}_{p} .
\end{gathered}
$$

It follows from estimates (34E) and (35E) in Lemma E.6 that the constant vectors $\mathfrak{F}_{\beta}, \mathfrak{F}_{\lambda}, \mathfrak{F}_{p}$ and the linear functionals $\mathcal{M}, \mathcal{L}, \mathcal{P}$ admit the estimates

$$
\begin{gathered}
\left|\mathfrak{F}_{\beta}\right|+\left|\mathfrak{F}_{\lambda}\right|+\left|\mathfrak{F}_{p}\right| \leq c\left(\left|F_{2}\right|_{s}+\left|F_{3}\right|_{s}+\left|f_{5}\right|\right), \\
|\mathcal{M}|+|\mathcal{L}|+|\mathcal{P}| \leq c \varepsilon_{0}\left(\left|\boldsymbol{\mu}^{*}\right|_{s}+\left|\boldsymbol{\lambda}^{*}\right|_{s}\right) .
\end{gathered}
$$

Moreover, estimate (33E) yields the inequality

$$
\left|\overline{\mathfrak{F}_{\beta}}\right| \leq c\left|\bar{F}_{2}\right|
$$


Combining estimates (87E), (89E) and (92E), (93E) we arrive at the inequality

$$
\begin{array}{r}
\left|\mathfrak{F}_{\beta}\right|+\left|\mathfrak{F}_{\lambda}\right|+\left|\mathfrak{F}_{p}\right|+|\mathcal{M}|+|\mathcal{L}|+|\mathcal{P}| \leq c \varepsilon_{0}(|\overline{\boldsymbol{\lambda}}|+|\overline{\boldsymbol{\chi}}|+|p|)+ \\
+c \varepsilon_{0}\left(\left|\boldsymbol{\lambda}^{*}\right|_{s}+\left|\chi^{*}\right|_{s}\right)+c\left(\left|g_{1}^{*}\right|_{s}+\left|\mathbf{g}_{2}\right|_{s}\right) .
\end{array}
$$

¿From this and (91E) we obtain

$$
\begin{array}{r}
|\overline{\boldsymbol{\lambda}}|+|\overline{\boldsymbol{\chi}}|+|\overline{\boldsymbol{\mu}}|+|p| \leq c \varepsilon_{0}(|\overline{\boldsymbol{\lambda}}|+|\overline{\boldsymbol{\chi}}|+|p|)+ \\
+c \varepsilon_{0}\left(\left|\boldsymbol{\lambda}^{*}\right|_{s}+\left|\boldsymbol{\chi}^{*}\right|_{s}\right)+c\left(\left|g_{1}^{*}\right|_{s}+\left|\mathbf{g}_{2}\right|_{s}\right) .
\end{array}
$$

Choosing $\varepsilon_{0}$ sufficiently small we finally obtain

$$
|\overline{\boldsymbol{\lambda}}|+|\overline{\boldsymbol{\chi}}|+|\overline{\boldsymbol{\mu}}|+|p| \leq c \varepsilon_{0}\left(\left|\boldsymbol{\lambda}^{*}\right|_{s}+\left|\boldsymbol{\chi}^{*}\right|_{s}\right)+c\left(\left|g_{1}^{*}\right|_{s}+\left|\mathbf{g}_{2}\right|_{s}\right) .
$$

Next, equations 80Ea)-80Ec) imply the equalities

$$
\begin{gathered}
\boldsymbol{\partial} \boldsymbol{\mu}^{*}=-p \nabla w_{1}-\nabla g_{1}, \\
\boldsymbol{\partial} \boldsymbol{\lambda}^{*}+\boldsymbol{\Omega} \boldsymbol{\lambda}^{*}=\mathbf{g}_{2}-\mathbf{T} \boldsymbol{\mu}^{*}-\left(\mathbf{T} \overline{\boldsymbol{\mu}}+p \mathbf{W}^{\top} \mathbf{e}_{1}\right) \\
\boldsymbol{\partial} \chi^{*}=\left(\mathbf{S} \boldsymbol{\mu}^{*}+\mathbf{T}^{\top} \boldsymbol{\lambda}^{*}\right)+\left(\mathbf{S} \overline{\boldsymbol{\mu}}+\mathbf{T}^{\top} \overline{\boldsymbol{\lambda}}\right)
\end{gathered}
$$

Applying Lemma E.2 to the first equation and noting that the embedding of every Sobolev space into $\mathcal{A}_{\sigma / 2,0}$ is bounded we obtain

$$
\left|\boldsymbol{\mu}^{*}\right|_{s+3 n+4} \leq c\left(|p|+\left|g_{1}^{*}\right|_{s+4 n+6}\right)
$$

Next, applying Lemma E.3 to the second equation we obtain

$$
\left|\boldsymbol{\lambda}^{*}\right|_{s+n+1} \leq c\left|\mathbf{g}_{2}\right|_{s+3 n+4}+c\left|\boldsymbol{\mu}^{*}\right|_{s+3 n+4}+c(|p|+\mid \overline{\boldsymbol{\mu}}) .
$$

Applying Lemma E.2 to the third equation we obtain

$$
\left|\boldsymbol{\chi}^{*}\right|_{s} \leq c\left(\left|\boldsymbol{\mu}^{*}\right|_{s+n+1}+\left|\boldsymbol{\lambda}^{*}\right|_{s+n+1}\right)+c(|p|+|\overline{\boldsymbol{\mu}}+| \overline{\boldsymbol{\lambda}} \mid)
$$

Combining the obtained results we arrive at the inequality

$$
\left|\boldsymbol{\mu}^{*}\right|_{s}+\left|\boldsymbol{\lambda}^{*}\right|_{s}+\left|\boldsymbol{\chi}^{*}\right|_{s} \leq c\left(\left|g_{1}^{*}\right|_{s+4 n+6}+\left|\mathbf{g}_{2}\right|_{s+4 n+6}\right)+c(|p|+|\overline{\boldsymbol{\mu}}+| \overline{\boldsymbol{\lambda}} \mid) .
$$

Combining (95E) and (96E), and choosing $\varepsilon_{0}$ sufficiently small we obtain the desired estimate

$$
|\boldsymbol{\mu}|_{s}+|\boldsymbol{\lambda}|_{s}+|\boldsymbol{\chi}|_{s}+|p| \leq c\left(\left|g_{1}^{*}\right|_{s+4 n+6}+\left|\mathbf{g}_{2}\right|_{s+4 n+6}\right)
$$


which gives estimate (8.10) in Theorem 8.1. Next, estimates (88E) and (94E) yields

$$
\left|\overline{\mathfrak{F}_{\beta}}\right| \leq c \varepsilon_{0}|p|+\left|\overline{\mathbf{g}_{2}}\right|
$$

which along with (91Ea) and (93E) implies

$$
|\overline{\boldsymbol{\mu}}| \leq\left|\overline{\mathbf{g}_{2}}\right|+c \varepsilon_{0}\left(|p|+\left|\boldsymbol{\mu}^{*}\right|_{s}+\left|\boldsymbol{\lambda}^{*}\right|_{s}\right)
$$

Combining this result withy (97E), we finally obtain

$$
|\overline{\boldsymbol{\mu}}| \leq\left|\overline{\mathbf{g}_{2}}\right|+c \varepsilon_{0}\left(\left|g_{1}^{*}\right|_{s+4 n+6}+\left|\mathbf{g}_{2}\right|_{s+4 n+6}\right),
$$

which gives estimate (8.11) in Theorem 8.1. This completes the proof of Theorem 8.1.

\section{References}

[1] Arnold, V.I., Proof of a Theorem of A. N. Kolmogorov on the Invariance of Quasi-Periodic Motions Under Small Perturbations of the Hamiltonian, Usp. Mat. Nauk, 1963, vol. 18, pp. 13-40.

[2] Arnold, V.I., Kozlov V.V., A.I. Neischtadt, Mathematical Aspects of Classical and Celestial Mechanics, Springer Science \& Business Media, 2007

[3] Bolotin S.V., Treschev D.V., Remarks on the Definition of Hyperbolic tori of Hamiltonian Systems, Regular and Chaotic Dynamics, 2000, vol.5, pp. 401-412. 
[4] Broer, H.W., Huitema, G.B., and Sevryuk, M.B., Quasi-Periodic Motions in Families of Dynamical Systems, Order Amidst Chaos, Lecture Notes in Math., vol. 1645, Springer-Verlag, Berlin, 1996.

[5] Cheng, C.-Q., BirkhoffKolmogorovArnoldMoser Tori in Convex Hamiltonian Systems, Commun. Math. Phys., 1996, vol. 177, pp. 529559.

[6] Cheng, C.-Q., Lower Dimensional Invariant Tori in the Regions of Instability for Nearly Integrable Hamiltonian Systems, Commun. Math. Phys., 1999, vol. 203, pp. 385419.

[7] Cheng, C.-Q., Minimal Invariant Tori in the Resonant Regions for Nearly Integrable Hamiltonian Systems, Transactions AMS, 2005, vol. 357, no. 12 , pp. 50675095.

[8] Corsi L., Feola R., Gentile G., Low-Dimensional Invariant Tori for for Perturbations of a Class of Non-convex Hamiltonian Functions, Journal of Statistical Physics, 2013, vol.150, pp. 156-180.

[9] Eliasson, L.H., Perturbations of Stable Invariant Tori for Hamiltonian Systems, Ann. Scuola Norm. Sup. Pisa, 1988, vol. 15, pp. 115-147.

[10] Graff, S.M., On the Continuation of Hyperbolic Invariant Tori for Hamiltonian Systems, J. Diff. Eqns., 1974, vol. 15, pp. 1-69.

[11] Kolmogorov, A.N., On the Conservation of Conditionally Periodic Motions for a Small Change in Hamiltons Function, Dokl. Akad. Nauk USSR, 1954, vol. 98, pp. 527-530. English translation in: Lectures Notes in Physics 93, Springer, 1979.

[12] Kuksin, S.B., Hamiltonian Perturbations of Infinite-Dimensional Linear Systems with Imaginary Spectrum, Funktsionalnyi Analiz $i$ ego Prilozheniya, 1987, vol. 21. no. 3, pp. 22-37.

[13] Li, Y. and Yi, Y., Persistence of Lower Dimensional Tori of General Types in Hamiltonian Systems, Trans. Amer. Math. Soc., 2005, vol. 357, pp. 15651600.

[14] Melnikov, V.K., On Some Cases of Conservation of Conditionally Periodic Motions Under a Small Change of the Hamilton Function, Soviet Math. Doklady, 1965, vol. 6, pp. 15921596. 
[15] Nirenberg, L., Topics in Nonlinear Functional Analysis, AMS, 2001.

[16] Percival, I.C., A Variational Principle for Invariant Tori of Fixed Frequency, J. Phys. A: Math. Gen., 1979, vol. 12, no. 3, pp. 57-60.

[17] Plotnikov, P.I., Morse Theory for Conditionally-Periodic Solutions to Hamiltonian Systems, Siberian Math. J., 1994, vol. 35, pp. 590-604.

[18] Poincare, H., New Methods of Celestial Mechanics, History of Modern Physics and Astronomy 13, Amer. Inst. Phys. 1993.

[19] Moser, J., Convergent Series Expansions for Quasi-periodic Motions, Math. Ann., 1967, vol. 169, no. 1, pp. 136-176.

[20] Poschel, J., On Elliptic Lower Dimensional Tori in Hamiltonian Systems, Math. Z., 1989, vol. 202, pp. 559-608.

[21] Treschev, D.V., The Mechanism of Destruction of Resonance Tori of Hamiltonian Systems, Math. USSR Sb., 1991, vol. 68, pp. 181-203.

[22] You, J.G., Perturbations of Lower Dimensional Tori for Hamiltonian Systems, J. Differential Equations, 1999, vol. 152, pp. 1-29.

[23] Zehnder, E., Generalized Implicit Function Theorems with Applications to Some Small Divisor Problems, Comm. Pure Appl. Math., 1975, vol. 28, no. 1, pp. 91-140; 1976, vol. 29, no. 1, pp. 49-111.

[24] Zehnder E., An implicit function theorem for small divisor problems, Bull. Amer. Math. Soc., 1974, vol. 80, pp. 174-179 